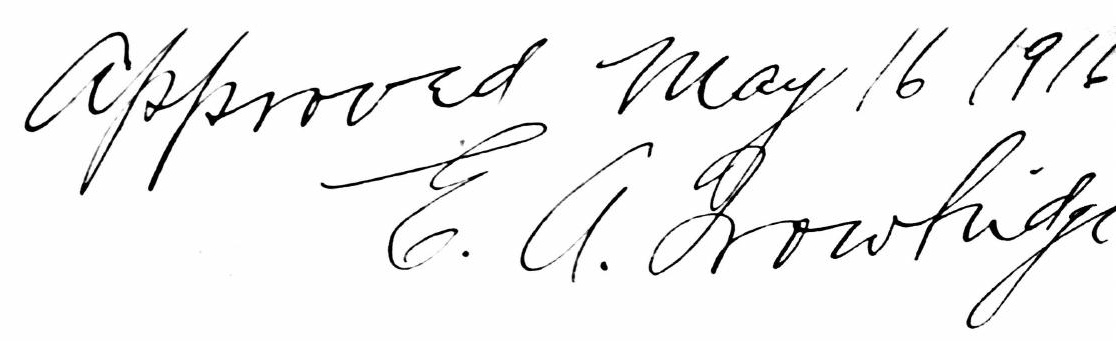

SILAGE AS A PART RATION FOR HORSES AND MULES OF VARIOUS AGES UNDER

VARYIIG CONDITIONS

$\operatorname{lig}$

BLUEA HOTARD HUGHES, B. S. IN AGR.

SUBIITTED IN PARTIAL FULFILUENM OF TEE REQUIREMENTS FOR THE DEGRES OF WASTER OF ARTS

in the

GRADUATE SCHOOL

of the

UNIVERSITY OF MISSOURI 
Introduotion $\ldots \ldots \ldots \ldots \ldots \ldots \ldots \ldots \ldots \ldots \ldots \ldots \ldots \ldots \ldots$

Ilterature $\ldots \ldots \ldots \ldots \ldots \ldots \ldots \ldots \ldots \ldots \ldots \ldots \ldots \ldots \ldots . \ldots \ldots$

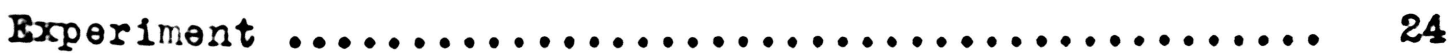

object $\ldots \ldots \ldots \ldots \ldots \ldots \ldots \ldots \ldots \ldots \ldots \ldots \ldots \ldots \ldots \ldots$

Plan $\ldots \ldots \ldots \ldots \ldots \ldots \ldots \ldots \ldots \ldots \ldots \ldots \ldots \ldots \ldots . \ldots \ldots$

Animals Used $\ldots \ldots \ldots \ldots \ldots \ldots \ldots \ldots \ldots \ldots \ldots \ldots . \ldots \ldots$

Rations Used $\ldots \ldots \ldots \ldots \ldots \ldots \ldots \ldots \ldots \ldots \ldots \ldots \ldots . . \ldots 29$

Plan and Hethod of Feoding ............... 29

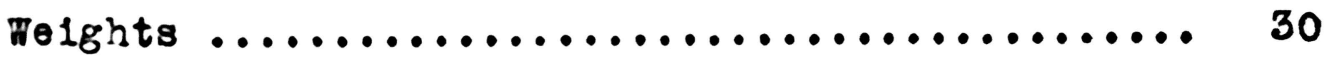

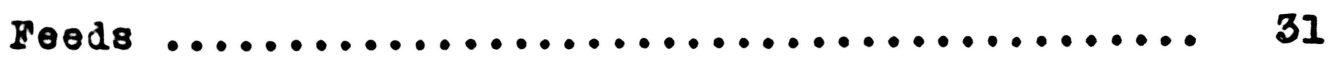

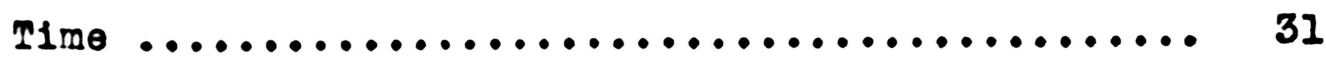

Exere1se $\ldots \ldots \ldots \ldots \ldots \ldots \ldots \ldots \ldots \ldots \ldots \ldots . \ldots \ldots, 32$

Trater .............................. 32

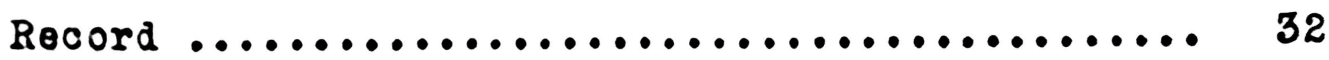

Da1ly Program .......................... 33

The 1914-1915 rest ......................... 34

Chemists' Data .......................... 34

Description of Horses in the 1914-1915 Test .... 40

Mangement during the summer and fall previous

to the beginning of the test ................ 40

Foed Recolved .......................... 41

Digestible Nutrients recoived .............. 42

Tables, Discussions and Photographs of Horses in

the 1914-1915 test ...................... 
Summary of total feed consumed during

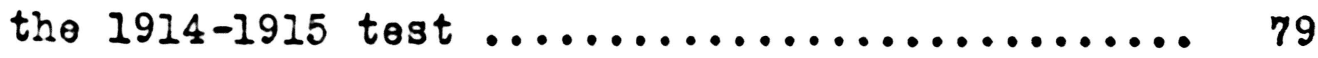

Summary of everage feed consumed during the

1914-1915 test ......................... 80

Summary of total digestible nutrients consumed

during the $1914-1915$ test ................. 82

Sunmary of average daily digestible nutrients

consumed during the $1914-1915$ test .......... 83

Summary of digestible nutrients consumed per

thousand pounds live weight during the

$1914-1915$ test ............................ 85

Sumary of average daily digestible nutrients

consumed per thousand pounds live weight during

the $1914-1915$ test ...................... 86

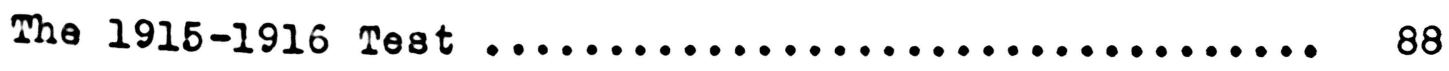

Introduction $\ldots \ldots \ldots \ldots \ldots \ldots \ldots \ldots \ldots . \ldots \ldots$

Chemists' Dats .............................. 89

Description of Horses in the 1915-1916 Test .... 94

Wanagement during the summer and fall previous

to the beginning of the test ............... 95

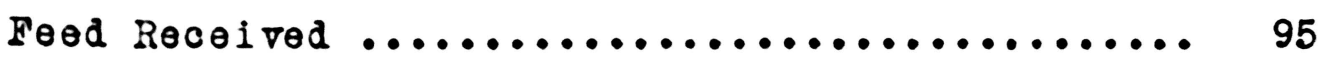

Digestible Nutrients Received .............. 96

Tables, Discussions and Photographs of Horses

in the 1915-1916 Test ................... 97

Summary of total feed consumed during the

$1915-1916$ test ......................... 144

Summary of average leed consumed per day during 
Summary of total digestible nutrients consumed during the 1915-1916 test ................ 147 Summary of average digestible nutrients consumed during the 1915-1916 test ................. 148 Summary of total digestible nutrients consumed per thousand pounds live weight during the

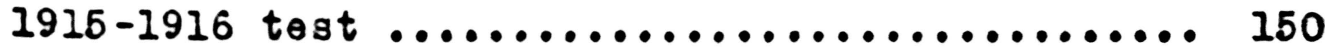
Sumary of average dally digestible nutrients consumed per thousand pounds live weight during the $1915-1916$ test $\ldots \ldots \ldots \ldots \ldots \ldots \ldots \ldots \ldots \ldots \ldots \ldots 15$ Summary of the 1914-1915 and the 1915-1916 tests .... 153

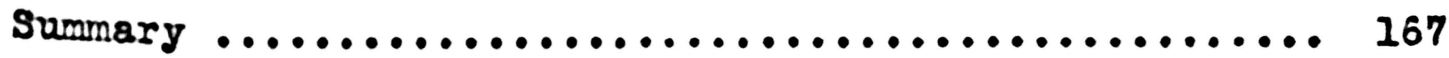

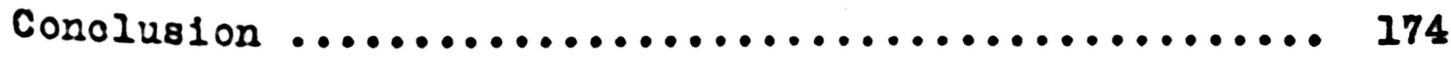
Suggestions for future investigations ............ 175 


\section{INTRODUCTION}

There were 21,166,000 horses and 4,565,000 mules In the United States on January 1, 1916. The average value of horses was \$101.60 per head, making an aggregate value of $\$ 2,150,468,000,00$ and the average value of mules was $\$ 113.87$. making an aggregate value of $\$ 519,824,000.00$.

On January 1, 1911 there were $20,277,000$ horses valued at $\$ 111.45$ per hoad, making an aggregate value of $\$ 2,259,981,000.00$ and at the same time there were 4,323,000 mules ralued at $\$ 125.92$ making a total valuation of $\$ 544,359,000.00$.

During the five period the estimated number of horses has 1ncreased 889,000 head but they have decreased in value \$9.86 per head. Their aggregate value has decreased $\$ 109,513,000,00$.

The estimated number of mules has increased 342,000 during the same period, but they have decreased in value \$12.05 per head. Their aggregate value has decreased $\$ 24,535,000.000^{1}$

The economic factors that have brought about these deoreases in value per head of horses and mules are not quite clear. Motor power has supplanted horse power in some cases and this has oqused a decreased demand for some types of horses. The increased cost of ford caused by the high price of land and labor and other oconomic factors, with an arerage 
decreased value per head of horses and mules during the past five joars makes it nocessary that the cost of production and maintenanoe of horses be cheapened wherever possible. The increased number of 81108 and a better lonowledge of their uses is providing farmers with an excellent cheap feed in the form of com silage. If this raluable roughage can be fed to horses with success its use will become a factor in cheapening the production and maintenance of horses and mules.

Eridence secured by the Department of Animal Husbandry by correspondence and from the agricultural press indicates that numerous farmers and breeders have for many years successfully fod corn silage as a part ration to horses and mules. These men have show great care in their feeding operations. They have fed silage to horses and males of all ages, including growing horses, brood mares, and working horses, With a riew to cheaponing the cost of growth and maintenance during the winter months.

The men who have been suocessful in feoding this roughage have been very careful to feed 8 ilage of good quality made from comparatively mature corn. It has been fed in varying amounts of from five to thirty pounds per hoad daily, the amount consumed depending somerhat on the size of the horses, the amount and kind of work performed, the quantity of grain and hay fed and upon the individuality of the horse. The afrioultural press ${ }^{2}$ prior to 1912 oationed 
against the use of silage, a few horses had died because of eating moldy, frozen or rotten silage. Since that time the press has taken a more liberal view of the situation and in most cases has recommended its use in limited quantities, cautioning against the feeding of moldy, frozen or rotten sllage, or silage made from immature corn.

Very little experimental work has been done to determine the best methods of leeding silage either as concerns the quantity to feed or the most desirable combinations with other feeds. Several experiment stations have fod it in a limited way to horses and mules of different ages under varying conditions.

An experiment was started at the Missouri Experiment Station in Decomber 1914 to investigate the value of silage as a feed for horses and mules of various ages. This investigation is to continue until sufficient data is collected to make defint te conclusions possible.

The purpose of this thesis is to study the results of this experiment, to summarize the data, and to make possible conclusions, which may be of value to 10,000 s1lo owners in Mlssourl and to the large number of farmers in the United states who have this feed at hand.

The date here used corers two twelre-week periods during the winters of $1914-15$ and $1915-16$ respectively. The general management of the horses was similar during both trials. The feods were of practically the same quality except that the corn sllage fed in the first trial was 
of much better quality than that fed in the second trial. Everg preoaution was taken to make both years comparable. An inquiry from this station was sent to all experiment stations in the United States, asking for information on silage as a feed for horses. Virginia, North Carolina, Pennsylrania, Wisconsin and Missouri reported experimental orldence. The Michigan and Tennesser experiment stations have fed silage to horses but have no records to show their results. Illinois, Iowa, Nebraska, Minnesota, Colorado, Texas, Kansas, California, Oklahoma, Kentucky, Georgia, Arizona, Arkansas, Washington and oregon made general statements of its value as a feed for horses. The following stations have no information: Loulsiana, Flor1da, Maine, Mississippi, Montana, New Hampshire, Massachusetts, New Jersey, North Dakota, South Dakota, Alabama, Ohlo, Rhode Island, Connecticut, Delaware, West Virginia, New York, Utah, How Mexico, and South Carolina. 


\section{IITERATURE}

The arailable literature on this subject divides Itself lnto five general parts. Experiment Stations and Colleges - Verg fer experiment atations have taken up the problem of feeding silage to horses and mules, hence experimental data and information is meager. ID. 0 . Nourse was the first man to feed silage to horses and mules experimentally. His work was done prior to the jear 1900. The experiments were undertaken because of a soarcity of forage in certain sections of Virginia, a diffoulty whioh he hoped to alleviate by the use of corn silage. silage was fed to two horses and six mules. They were given small quantities of silage for some time before the test proper. seter the preliminary period they were fed for $81 \times$ weeks on a ration consisting of all the silage they would oat with hay and corn in addition.

Two lots of horses and mules were fed the following rat1ons :

$$
\begin{aligned}
& \text { a. Corn silage, corn and hay } \\
& \text { b. Corn and hay }
\end{aligned}
$$

The gains in weight of both lots were about equal. The animals were oonstantly at work except during stormy weather. It would appear from the results obtained that corn sllage would make a desirable roughage for horses when fed in confunction with grain, hay or stover. The animal should beoome 
acoustomed to it by degrees.

IT. 1. Henry, Dean and Director of the Wisconsin Bxperiment Station, early in the jear 1902 expressed a desire that some horsemen try corn silage as a feed for horses. He suggests moderation in its use for joung animals, brood mares and 1dle horses. He presented a discussion of its economio value in feoding farm animals.

\section{C. W. Burkett at the North Carolina station fed} horses and mules belonging to the college, to compare local grow feoding stuffs with each other and with purchased feeds. He concluded, "Corn silage is a superior feed for horses and mules." One of the most satisfactory rations fed in this series of experiments was composed of 21 pounds corn sllage, 15 pounds corn, 2 pounds bran, and 1 pound cotton seod meal. The ration proved to be cheap, efficlent and wholesome to the animals. B. G. Ritzman reports that, ${ }^{3}$ "silage from para grass has boen fed to mules. When fed with about one liter of cane molasses diluted in water and sprinkled throughout they ate 1t, but when molesses was not mixed with the silage they apparently cared little for it."

4. A. Coohel at the Pennsylvania Experiment station fod s1lage to horses. The object was to use the common feeds,

\footnotetext{
I Breoders Gazette, May 14, 1902

Ellorth Carolina Exp. Station Bulletin No. 189

3

Report of the Assistant Animal Husbandman, Porto Rico Station Roport, 1908, pp. 37-39

4 Penneglrania Exp. Station Bulletin No. 117
} 
corn, oats and mixed hag as the basic ration, to find whether cottonseed meal could replace oats and cheapen the cost, and to flnd further if 81 lage could not be substituted for a part of the hay in the ration thus cheapening the cost of fattenIng horses.

Twenty-one geldings and mares were used. They were olassified on the market as "ohanks" or "light draft." The horses were divided into three groups of seren each as nearly alike as possible with referenoe to size, conformation, eto., and were fed for a period of 84 days.

\section{Rations Ped}

Group 1, Corn, oottonseed meal, corn silage, mixed hay

" 2, Corn, cottonseed meal, mixed hay

n. Corn, oats, mixed hay

The grain ration which started at 8 pounds per day was increased one pound per day for the first week. Hay was fod acoording to the appetite of the individual horse. Silage was fod in Group 1 at the rate of 5 pounds per head dally at the beginning which was gradually increased to 20 pounds daily. Prom the results of the test the following conclusions were arawn:

1. S1lage which $18 \mathrm{made}$ Irom mature corn, is Ireo from mold, has not been exposed to air too long before feeding, and is properig expplemented with other feeds which will make up the defloienoy in protein, can be fed to horses with safety when care is used to have them become gradually accustomed to it. 
2. Horses fod silage as a part of their ration consumed less grain, made their gains at less cost per pound, were sleeker and better finished than when fed on rations not containing silage.

1T. A. Cochel and B. O. Severson report an experiment during the whter of 1910-1911 in which ten grade Percheron and Belgian colts were divided into two groups. One group of four colts recelred corn silage as a part ration. The other colts recelved no silage.

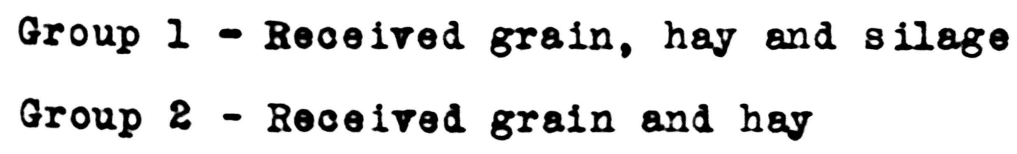

The grain mixture was composed of :

5 parts shelled corn

3 parts oats

2 parts bran

1 part oil meal

During the first three months of the winter Group I was fod:

5 pound of grain per head daily

7.4 pounds hay per hoad dally

8.2 pounds 81 lage per head daily

Group 2 was fed:

5 pounds grain per hoad dally 10.6 pounds hay per head dally Group 1 rocoived silage in the morning and hay at 
night.

During the second period of three months the grain ration was increased 7.5 pounds and the hay to 13.1 pounds per head dally in each group. ' With this method of feoding all the oolts oame through the winter in excellent condition of llesh, hering gained 244.7 pounds per head during the winter feoding period of 168 daje.

In order to try $81188 \theta$ as a part ration for growing I0als an attempt was made at the "Wisconsin Experiment station to feed 81188 to three draft weanlings during a four week period from November 11, 1911 to December 9, 1911.

The 11 1lage was fed in pails apart from the regular ration. Two of them were given one pound daily during the time and the third was fed the ame guantity from Hovember 24, 1911 to December 9, 2911. It was fed in addition to the regalar ration, fresh silege of good quality belng given at each loed. At no time did they oare for it and usually ignored it entirely. After trying it for same time and not suoceeding in getting the foals to eat the 81lage, the 1dea of lncluding it as a part of the ration was given up.

It was erident that there was too great a contrast between the s1lage and the regular ration, silage being rather bltter and having an acid taste, while the regular ration was somewhat sweet. The forls were unacoustomed to ang feed of this nature and, since they were receiving abundant nutriment

${ }^{1} 8118 g 0$ was not fed to Group 1 during the second period EThompson and Bughes, Thesis University of Wisconsin, 1918 
and a supply of variety from the regalar ration, they did not oare for the additional feed.

F1vo draft gearlings, averaging 1050 pounds in woight were started on feed september 28, 1912. ${ }^{1}$ Having been on pasture during the previous summer without grain they were in thin condition but growthy. They were fed a ration of $60 \%$ ground oats $30 \%$ bran and $10 \%$ chaffed olover hay for about throe weoks prior to the beginning of the test.

Although a good quality of s1lage was fed, some difficulty was experienced in getting them to eat it at first. The leaver which seemed to contain the least acid were consumed and pieces of the cob with kernels of corn on them were loft.

They were started on a ration of 12 pounds ground oats, 8 pounds bran, and 3 pounds of chaffed clover hay, mixed With 22 pounds of corn silage.

Elghteen to twenty pounds of this mixture was consumed daily. The amount of 8 il ago was gradually increased until at the end of twenty days they were receiving from sixteon to tronty pounds per head dally. When on full silage leod they were getting a ration composed of $65 \%$ silage, $21 \%$ ground oats and $14 \%$ bran. They conoumed from thirty-five to thirty- ight pounds of this mixture per head daily. This ration was fed steadily for forty-nine dags. The bran was discontinued at that time beoause the ration contained two laxative feeds, 
bran and 811880, which did not prove satisfactory in the quantities fod. The ration contained $65 \%$ corn silage and $35 \%$ ground oats. Hag was fed in the racks when they were on full siloge leod.

The last ration was fed for a period of three weoks, at the ond of which two of the animals were changed to a grain ration of $75 \%$ ground oats and $25 \%$ bran. In a week another jearling was changed to the same ration. The other two horses reoelved the ration containing silage for the following three weoks. They were then given a ration of $60 \%$ ground oats, $20 \%$ ground corn and $20 \%$ alfalfa hag. The feedIng of the last ration resulted in substantial gains in weight. silage did not prove satisfactory in the quantities fed. This was due perhaps to the large amount of silage that was fed. It was estimated that they did not gain more than one-half the amount possible had they been fed a more 1deal ration.

1B. A. Trowbridge reports having fed ten jearling mules weighing approximately 650 pounds each for 90 days on an average dally ration of 6.5 pounds of oar corn, 8.6 pounds of mixed hay and 4 pounds of corn silage. The males made $a$ bain of only 4.8 pounds during the period. Ho 111 results were observed from the use of the silage but the mules did not consume large quantities of it. This, it is thought, mas 
have been due to the fact that the silage was made from rather immature corn. It is adrised that corn silage be fed in combination with other feeds and that under no circumstances should spoiled silase, either moldy or rotten, be fea to horses or mules.

The Miohigen ${ }^{2}$ and Tennesser ${ }^{2}$ Experiment Stations have been feoding silage to horses for a number of jears. They have found it eoonomical and safe to feed in quantities of from fifteen to twenty pounds of good silage per head dally. They have no arailablo data.

Digest of information on 811 age for horses and mules taken Prom recent publications ${ }^{3}$ - Corn sllage mag be safely fed to horses and mules in moderate quantities provided it is of good quality. It 18 one of the cheapest and best feeds for ide horses, brood mares and horses that are doing light work during the winter months.

It should bo fed in small quantities at first and the amount gradually increased until the animal becomes acoustomed to 1t, when a maximum feed of from 10 to 20 pounds may bo given daily. It should be fed only where intelligent sppervision insures the use of silage of good quality.

IR. S. Hudson, Miohigan Exp. Station E. A. W1llson, Tennessee kxp. Station $3_{\text {Honry }}$ and Morrison, Feeds and Foeding, 15 th edition. 7. W. Woll, Productive Feoding of Farm Animals T. H. Jordan, The Feoding of Farm Animals 
United States Department of Agriculture - Ingilago was fed in large quantities last winter to some of the Morgan mares on the Government Farm in Vermont, and the results were satisfactory. These mares wore left out during the entire winter until fust before foaling time in the spring. In open shed afforded protection from storms. The daily ration per mare was 20 pounds of silage and 10 pounds of hag. The attendants report that the meres came thru the winter in better condition than those of a check lot wintered under similar conditions but with a grain ration, and their foals when dropped were all straight and sound."

"During the winter of 1915-1916 Morgan mares weighing 1,000 pounds at the Government Yarm, ${ }^{2}$ Middlebury, Vermont, were fod as much as 24 pounds of 8 ilage per head. These mares were not working other than raising their foals.

"Corn 81 lage has been fed to work stock at the Government Farm, ${ }^{2}$ Beltsville, Margland, during the past two winters. These horses average about 1,400 to 1,500 pounds in weight and have been receiving ten to twelve pounds of corn silage a day. The work which they have been performing during the winter months has been verg light. Our experience has been that a part of the roughage oan be economically made up of silage."

1

Breoders Gazette, Pebruary 5, 1913

8 Correspondence, April 4, 1916

G. Arthur Bell, Senlor inimal Husbandman 
The following is an extract from Farmers' Bulletin No. 578. It is presented because of the general information it contains on the subject;

G00. Zommel, U. S. Department of Agriculture

$$
\text { Farmers' Bulletin No. } 578
$$

\section{S1lage for Horses}

Sllage has not been generally fed to horses, partly on acoount of a certain amount of danger which attends its use for this purpose, but still more, perhaps, on account of prejudice. In many cases horses have been killed by eating moldy 81 lage and the careless person who fed it at once blamed the sllage 1trelf, rather than his own carelessness and the mold which really was the cause of the trouble. Horses are peculiarIf ouscoptible to the effects of molds, and under certain conditions certain molds grow on silage which are deadly poisons to both horses and mules. Molds must have air to grow and therefore sllage which is paked air tight and fed out rapidly will not become moldy. If the feeder watches the silage carefully as the weather warms up he can soon detect the presence of mold. When mold appears, feoding to horses and mules should stop Immodiately.

It is also unsafe to feed horses frozen silage on acoount of the danger of collo. This is practically impossible to aroid in very cold weather, especially in solld wall silos. By taking the dags foed from the unfrozen conter of the 8110 and ohopping away the frozen silage from the odges and piling the frozen pieoes in the center the mass will usually thew 
out in time for the next feed.

Corn to be made into silage for horses should not be cut too green as sour silage will result and mag cause colic when fed. The corn should be well matured and cut when the grain is beginning to glase. The silo should be filled rapidIf and the corn should be vigorously tramped and packed while filling. At least three men should be inside the silo, moving constantly, two around the edges and the third across and around the center. This 18 by far the most important in connection With feeding silage to horses, and the lires of the horses fed on silage may depend on the thoroughness with which the trampIng is done. If properly done no danger is likely to result; if not properly done air pockets may form and cause a small mass of mold which the feoder may orerlook but which might be suffiolent to kill one or more horses.

The ralue of silage for horses is greatest as a means to carry them through the winter season cheaply or to supplement pasture during drought. As the danger of mold is greater In summer than in winter, silage should not be fed to horses In that season unless a large number of animals are getting it, and the daily consumption is 80 large as to preclude its formation on the surface.

To cheapen the ration for brood mares in winter no feed has more value than good corn silage. If the grain goes into the s1lo with the stover no additional grain is needed for brood mares, hay being the only supplementary feed necessars, 
If there is littie grain on the corn the silage should be supplemented with I pound of old process linseed oll meal or cottonseed meal daily per 1,000 pounds live weight. Horses to be wintered on a silage and hag ration should be started on about 5 pounds of silage daily per 1,000 pounds live weight, the grain and hay ration being gradually decreased as the silage is increased until the ration is 20 pounds silage and 10 pounds of hay daily per 1,000 pounds live woight. It will require about a month to reach the full feed of silage but the perfod may bo decreased somewhat, depending on the fudgment and skill of the feeder.

Mares fed in this manner will be in splendid condition for foaling and so far as the writer's experience goes the fools will be fully as vigorous, with just as much size and bone, as if the mares were fed the conventional grain and hay ration.

Work horses when idle can be wintered satisfactorily In this manner, but much silage is not recommended for horses that do heapy work for the same reason that a driving horse cannot do his best while on watery grass pasture.

The writer knows of cases where stallions receive a ration of silage but has had no experience in feeding them in this manner. There seems no reason why silage should not be a valuable feed for stallions during the idle season.

silage should also be useful for young horses, especially drafters, but here ogain the writer cannot quote 
his own experience and experimental data are megger.

To summarize, silage is safe to feed to horses and mules only when it is made from fairly mature corn, properly 8tored in the 8110. When it is properly stored and is not allowed to mold, no feed excoeds it as a choap winter ration. It is most valuable for horses and mules which are not at heary work, such as brood mares and work horses during the slack season. Whth plenty of grain on the cornstalks, horses Will keop in good condition on a ration of 20 pounds of silage and 10 pounds of hag for each 1,000 pounds of live weight.

"It is atated that corn silage has been satisfactorily used at the lool River Remount Depot as leed for horses. The daily ration for horses running in paddocks consisted of 4 pounds of choppod com silage, 2 pounds of alfalfa or forage, a few pounds of crushed corn and an ounce of salt. From the first they liked the ensilage and did reasonably well on it. Many horses not doing well before showed improvement. The dropplings were of a partioularly healthy nature. The stabled horses also had a few pounds of sllage mixed with other feed."

Inporiment Station Record No. 18, p. 969 National Agricultural Journal and Univ. Rec. 9 (1906), No. 12, pp. 1123-1128 
Answers to Inquiries - Sixteen replies were received from silo owrers in Carroll County ussouri in answer to an inquirg sent out from the Wissouri Experiment station. They report having fed sllage to horses and mules from one to five years, in quartities ranging from five to thirty pounds per head daily. With one exception, favorable results were obtained. This man states that some of his mares lost their colts, but he had no definite proof that the silage was the cause. He reports good results from its use with growing and working horses. Its use was heartily recommended with this exception. Other inquiries were sent to men in uissouri and in other corn belt states. Replies were received from men who have been feeding silage from one to twenty-five years. They report favorable results without exception. Work horses, mules, growing horses, brood mares, and draft stallions have been fed from five to twenty-five pounds of silage per head daily. They state that silage of good quality, made from comparatively mature corn was fed.

\section{$I_{G}$. N. Uihills has fed silage for twenty-live jears} to an arerage stud of one hundred Percheron horses. Aged stallions, used for breoding purposes, are fod during the winter months about twenty-four pounds of silage dally in conjunction with hay and grain. No silage is fed during the breoding season because of the difficulty of getting mares in foal. Two year old stallions receive about twenty pounds and 
the jearlings about fifteen pounds of silge per head daily. Mares with foals at side are fed about twenty pounds per day. This quantity is reduced to fifteen pounds after the foals are weaned.

Growing horses, including mares and geldings, are fed about fifteen pounds per head daily. Foals are fed a small quantity of silage while with their dams and after they are weaned are fed about seven pounds per head daily with grain. There has never been siokness among his horses which could be attributed to silage.

He olaims that his horses always come thru the winter In good breoding condition and that they are in proper shape to be turned upon spring pasture. Reports of County Farm Advisers - The Mssourl county farm advisers assert that in some sections of this state there are oomparatively few silos, but in communities where silos are numerous a large proportion of farmers have been foeding 81 lage to their horses and mules the past two to four jears. R. J. Howat in a survey of Saline County found that forty-one out of eighty-four silo owners led silage to horses and mules and with but one exception its use proved satisfactory. The owner who reported unfarorably had found no bad rosults, but thought it was not a good horse leed.

In Marion County, I. F.Gillmor found that one hundred farmers fed sllage to horees and mules during the winter of of 1914-1915. Of this number three reported unsatisfactory 
results, only one of whom was sure that silage was the oause. R. I. Cuff, county representative, Barron, Wisconsin, mentions the fact that both corn and pea silage is being fed by many farmers in that section. Silage is being substituted for a part of the hay in the ration. They consider that their horses come thru the winter in better condition than when fed on dry leed.

"Some canning companies in Wisconsin" winter their mules on pea vine sliege, ear corn and a little corn fodder. Thoir mules winter in good condition."

Reports in the Agricultural Press - Mang men have noted their personal experience feoding silage to horses in the Breeders Gazette.

Fletoher Brothers ${ }^{2}$ have led silage to horses since 1890. It was fed in conjunction with grain and hay to work horses and to range horses which were being broken and fitted for market. They are heartily in faror of feeding silage of bood quality to horses as a part of the ration.

"Since the preponderance of evidence reported to the Gazette ${ }^{3}$ strongly reoommends the feeding of silage to any kind of stock that will eat it, and since the per cent of $1088 \mathrm{~s}$ attributed to its uses is small, even where it has obviously been fed infudiciously, It would seem that there was not ground

IR. Baker, Ass't Seoretary Wisoonsin Live Stook Breeders' Ass'n $Z_{\text {Hennepin County Minnesota }}$ 3 Editorial, Breeders Gazette, March 15, 1911 
on which to oppose the careful feeding of a prime quality of silage to ang beast by which it is relished.

"Whatever danger may lurk in silage as a supplement to the rations of horses and mules is due in a large measure to the unfit condition of the corn at the time of siloing it. Frosted corn is often converted into silage for no other reason than that it is not adapted to any other use.

"It is chemically and physically unsuited to the purpose if a first grade of silage is desired. Even though it is properly prepared by the cutters and solidly packed into the silo it laoks the quality and natural integrity which the article must possess if it is to be fed without fear of unweloome results. Silage is of sufficient economic value to warrant the adoption of the most exacting methods in growing and handling corn for the special purpose of its production." "I There is no danger in feeding mules a reasonable amount of good corn silage with other grain and hay feeds. About one-half silage and one-half other grain or hay would be a good proportion.

"Moldy or spolled silage is dangerous for any class of Iive stock."

$"{ }^{2}$ Horse feoding has been my business for the past 30 joars and I now firmly believe that good corn silage is most valuable as a feed for all kinds of horses. With ordinary oare no $1088 \theta$ will be experienced. Very fow cases can be

II. M. Monsees, Breeders Gazette, September 24, 1913 ${ }^{2}$ Harry Birkett, Iincoln, Lancaster County, Nebraska Brooders Gazette, January 6, 1916 
absolutely traced in which horses have been killed by feeding 81lage.

I am now starting my fifth jear in feeding silage to draft horses. I was numbered with the doubting five jears afo and had to be strongly urged before I rould attempt to foed allage to high priced horses, but from the results obtalned the first season I became a firm believer in it.

The horses I am now feeding are all draft stallions ranging in ages from one to seven years, and in size from 1,200 to 2,400 pounds. In a large box I mix the following ration: 2 bushels of s1lage, I bushel of ground oats, and $1 / 2$ bushel of wheat bran. This would equal 44 pounds of silage, 22 pounds of 0 ats and 10 pounds of bran. The sil age is of good quality made from corn which would go at least 65 bushels per acre and being well matured when put in the silo. To a horse weighing 2,000 pounds I give three feeds a day of eight pounds each, making a total of twentg-four pounds. Between feeds the horses have access to good timothy hay, which they consume in proportions ranging from 6 to 12 pounds daily. They are fod in adition a small ration of corn, two to four pounds, depending upon their condition and needs. The averoge horse sets about fourteen pounds of siloge a daj. Some I feed as much as 18 to 20 pounds dailg.

I have noticed an absence of colic in our horses and our records will show that we have had less need for reterinary service sinoe we have been feeding silage. 
"I look upon good corn silage as a substitute for pasture. It tones up the animal, keeps the digestive tract in good order and undoubtedis aots as an appetizer. It is a cheap feed, and I believe one of the safest and best for breeding horses. It is excellent to mix with such feeds as bran and oats, which are dusty and tend to cause waste when fed in the ordinary way. By mixing such feeds with good corn silage the whole mass is moistened and the animals are very fond of 1 t.

"Care should be exercised in building the silo designod for horse foeding to have it narrow in diameter, thus giving a small feeding surface and plenty of opportunity for packing. The oorn should be well matured when put into the silo. It should be cut into small pieces and thoroughly packed. I am now feeding 50 draft stallions and there is not one of them but that is doing well on the silage ration. With ordinary care in using good silgge free fram mold the best results may be expeoted."

A summary of the results of experiment stations, colleges and the United States Department of Agricultare trials, the opinions of practical men who have expressed themselves from actual experience, the results of investigations by county agents and the opinion of the agricultural press, shows that corn silage of good quality made from corn that is comparatively well matured, properly put into the silo can be made part of the ration for horses and mules of various ages under varying conditions. 
It was with these facts in mind that an experiment was planned and carried out at the Missouri Experiment station to get more definite data on the sabject.

It is the object of this thesis to study the arailable data and to make definite conclusions in so far as that 18 possiblo.

\section{BXPERIUENT}

Silage as a part ration for horses and mules of various ages, under varying conditions.

\section{OB JECT}

The object of this experiment was to secure information conoerning:

a. The feoding value of corn silage when fed to horses and mules in conjunction with other feeds.

b. The most satisfactory combination of corn silage and other feeds for horses and mules of rarious ages and under varying conditions.

\section{PIAN}

\section{Groups and Lots}

The animals were divided into groups, basing such difision upon age, type, condition, labor performed, and any other factors which affect uniformity. Draft horses, mules and animals of lighter breeds were used. They were divided as follows :

Group A, Mature 1dlo horses and mules

$$
\text { a. Mrales }
$$


b. Geldings and open mares

o. Pregnant mares

Group B, Mature horses and mules performing average

winter farm work

a. Mules

b. Geldings and open mares

c. Pregnant mares

Group C, Growing horses and mules

a. Males

1. Poalo

2. Yearlings

3. Two Year 0lds

b. Draft Animals

1. Fo818

2. Yearlings

3. Two year 01ds

0. Animals of light breods

1. Foals

2. Yearlings

3. Two jear olds

Group D, Immature horses and mules performing average winter farm work

a. Two and three jear old mules

b. Two and three year old geldings and open mares

c. Two and three jear old pregnant mares

Group I. Immature idle horses and mules

a. Two and three jear old mules 
b. Two and three year old geldings and open mares

c. Two and three year old pregnant mares.

The horses and mules in each group were divided into two lots as unfformly equal as possible. Lot one receired a ration containing corn silage. Lot two receited a check ration in which no sllage was led. The lots were treated in the same manner except as to feed. The work performed by one horse in a team was the same as that performed by the other horse in the team. The teams were not separated during the tests. The quarters, the oollege horse barn and adoining lots, and care were as uniform and satisfactory as could be given.

\section{ANIMALS USED}

The animals used in the two winter trials were the horse and mule stook used, grown or kept by the Department of Animal Husbanary, College of Agriculture, University of Missouri. This stock included pure bred draft horses, grade draft horses, horses of light breeds and mules.

The horses and males used in the 1914-1915 test were:

Group 1

o. One pair of pure bred sadile mares in foal, Rath MoDonald and Martha 2nd

Group B

a. One team of mules, Jale and Kate

b. One team of grade draft geldings, Mack and Cap

o. Two teams of pure bred Peroheron mares in foal, Josephine and Forgette, Felibree and Finette 
Group C

b. Draft animals

1. One pair draft foals

c. Animals of light breods

1. Ono pair of saddle foals

2. One pair of light horses, Pepper, two years old, Lady Adrance, one year old

Group D

b. One team of pure bred Percheron mares, Josephe and Mena

Group E

b. One pair saddle mares in foal, Iona Rex, three jears old and Satelite, two years old

The horses used in the 1915-1916 test were:

Group A

b. One pair of light mares, June and Jill

c. Two pair of saddle mares in foal, Martha and and Ruth MoDonald, Satelite and Iona Rex

Group B

b. One team of grade draft geldings, Cap and Mack

c. One team of Percheron mares in foal, Felibree and Porgette

Group C

b. Draft animals

1. Two pair of draft foals

2. One pair of draft yearlings 
c. Animals of light breeds

1. One pair of light foals

2. One pair of light jearlings

Group D

o. One team of pure bred Percheron mares, Mena and Josepho

In addition to the animals grouped in teams or in pairs the following horses were fed silage as a part ration: Josephine, a pure bred Percheron mare not at work and thought not to be in forl. Jess, a grade draft mare at work. Jim, a grado draft gelding not at work. Magna, a pure bred saddle mare not in foal. Lady Adranoe, a two year old standard bred mare which was driven some. (\$ot in 108l) 
RATIONS USED IN THIS EXPERIMENT

\begin{tabular}{|c|c|c|}
\hline ROUP & IOT I & IOT II \\
\hline $\boldsymbol{\Lambda}$ & $\begin{array}{l}\text { *Grain } \\
\text { Alfalfa hay } \\
\text { S1lago }\end{array}$ & $\begin{array}{l}\text { Grain } \\
\text { Alfalfa hay }\end{array}$ \\
\hline B & $\begin{array}{l}\text { Grain } \\
\text { Timothy hay } \\
\text { Silage }\end{array}$ & $\begin{array}{l}\text { Grain } \\
\text { Timothy hay }\end{array}$ \\
\hline$c$ & $\begin{array}{l}\text { Grain } \\
\text { Alfalfa hay } \\
\text { S1lago }\end{array}$ & $\begin{array}{l}\text { Grain } \\
\text { Mlalfa hay }\end{array}$ \\
\hline D & $\begin{array}{l}\text { Grain } \\
\text { Timothy hay } \\
\text { S1lago }\end{array}$ & $\begin{array}{l}\text { Grain } \\
\text { Timothy hay }\end{array}$ \\
\hline $\mathbf{E}$ & $\begin{array}{l}\text { Grain } \\
\text { Alfalfa hay } \\
\text { S1lage }\end{array}$ & $\begin{array}{l}\text { Grain } \\
\text { Alfalfa hay }\end{array}$ \\
\hline
\end{tabular}

*Grain, Composed of 2 parts corn, 2 parts oats, and 1 part bran, bj woight.

Josephine, Jess and Jim received the same ration as horses in Group B, Lot I. Magna and Lady Adrance received the samo ration as horses in Group $\Lambda$, Lot $I$.

PIAN AND METHOD OF FHEDING

The object in foeding the mature horses whether idle or doing average winter farm work, including mules, geidings, open or pregnant mares, was to keep them in thrifty condition rather than to make them fat. The condition and needs of the 
Individual animal determined the amount of dry matter fod per 1,000 pounds live weight.

Draft horses and mules doing average winter farm work were led timothy hay. Growing horses and mature horses not dolng average winter farm work were fed alfalfa hay. Josephine and J1m, mature horses not at work were fed timothy hag.

The grain, including 2 parts corn, 2 parts oats and 1 part bran, by woight, was thoroughly mixed before feeding.

The horses fed silage as a part ration in the 19141915 trial were fod s1lage in the morning and hay at night. In the 1915-1916 test the siloge fed horses received silage at night and hay in the morning. Horses not fed silage receired hay at night and in the morning.

The amount of 81 lage that was fed depended upon the 81ze, age, work performed and upon the individurlity of each animal in the different groups.

Mature and immature horses doing average winter farm work were fed grain three times per day except sunday when they were lod the grain mixture twice per day, morning and night. Mature and immature horses doing arerege farm work received a larger grain ration than did the idle horses.

\section{WEIGHTS}

The horses were weighed weekly. The infial weights and the final weights consist of weights taken on threo successiro daje, the second of which in each case was the beginning 
and the closing date of the trial. The weights were taken in the morning before feed or water was given. This was done to minimize fluotuations in weights. All feeds were weighed for each Individual and feed refused was weighed back and deduoted Prom the amount given.

\section{PEKDS}

The grain fed consisted of corn, oats and bran which wore purchased on the open market. The corn fed was graded as "Number two mixed." The oats were graded as "Number two White Northern," and the bran fed was of good quality. The roughage consisted of alfalfa and timothy hay, and corn silage. The alfalfa hay and timothy hay were purchased on the open market and were graded as "cholce." The corn silage used in the 19141915 test was of excollent quality. It was made from corn that was comparatively well matured. It was estimated that the corn would field sixty-five bushels per acre.

The oorn s1lage fed in the 1915-1916 trial was of only fair quality. The summer of 1915 was extremely wet and part of the corn did not meture. The corn on the land whioh was well drained was very good, but the corn on the lower ground was poor, having small, partly filled ears. It was estimated that it would jield forty bushels per aore.

\section{TIMR}

The data to be presented were taiken from two tests. The Pirst test started December 24, 1914 and ended March 17, 1915. 


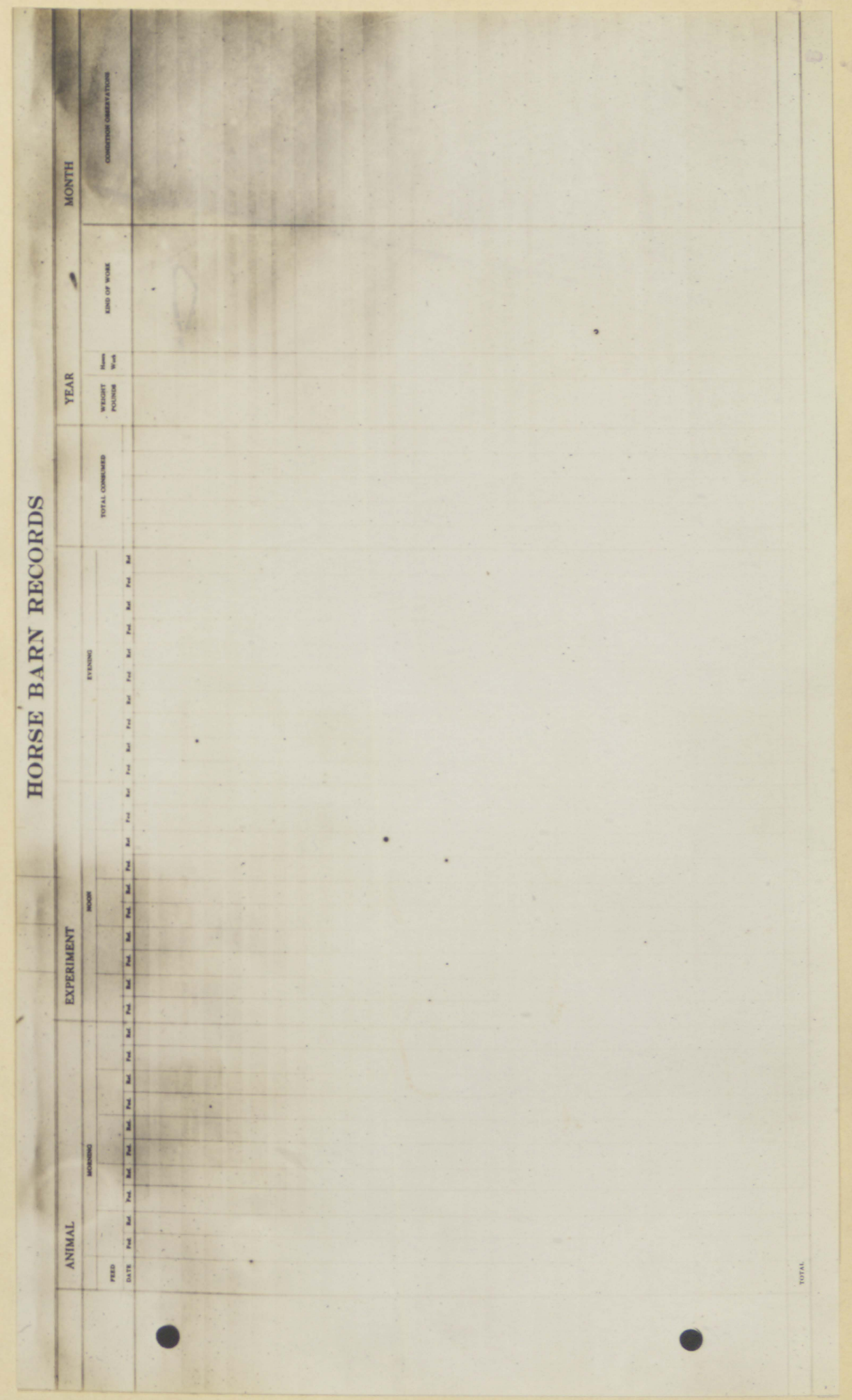


The second test started December 17, 1915 and closed March 10, 1916. These two trials extended orer two twolve week periods, making a combined total of twenty-four weeks in the two tests.

\section{EEDERCISB}

The labor performed by the animals doing average Winter farm work made other exeroise unnecessary. 111 other horses received exeroise in the lots adjoining the college horse barn except in case of inolement weather when theg were kept in the horse barn.

\section{WATER}

The horses and mules that were at work received fresh water three times daily. The other horses were watered at night and in the morning. Salt was kept before them at all timos.

\section{RECORDS}

Foed and woight reoords were kept for each individual animal during the tests. Attaohed is one of the sheets used in keoping these records. Descriptive records were made of oach Individual animal at the beginning and at the close of oach trial. Photographs of each individual animal and of each pair of animals were taken at the close of the 1914-1915 trial and at the beginning and close of the 1915-1916 trial. Feed Bamples - A smell quantity of each feed was put in a closed container each time that feed was mixed, which made a composite 
eample of each feed for each 12 reek period. The chemical analjals of the feod samples was made by the Agricultural Chemistry Department, University of Missourf.

\section{DAIIY PROGRAM}

6:00 A. M., weights taken once a week

$6: 15$ - 6:46 A. M., grain and roughage fed

7:00 - 7:30 A. M., watered

7:15 A. M. - 12 M., forenoon work

8:00 A. M., idle and growing horses turned into lots

12:00 M., horses at work watered and fed grain

1:00 - 5:30 P. M., afternoon work

6:00 P. M., horses in lots brought in and watered

$5: 30$ - 5:45 P. M., work horses watered

$5: 46$ - 6:00 P. M., grain and roughage fed 
THE 1914-1915 TEST 


\section{CHEMISTS' DATA}

It was impossible to run digestion trials in these teste hence the results of digestion trials as given in Henry and Morrison Feeds and Feeding, 1915 edition were used. No tests have been reported on the coefficient of digestibility of com silage and bran for horses. The coefficient of digestibility of these feeds by ruminants was substituted. "There would not be any wide differences in the digestion ooefficients of feeds such as silage and wheat bran for these elasses of animalo." 1

Timothy hay was fed to mature and immature draft horses and mules at work because of its energy value. It does not have the laxative effect that is characteristic of alfalfa. Alfalfa hay was fed to the mature light horses to make possible a reduction in the amount of grain in the ration. It was fed to all growing horses not at work because of the amount of digestible protein and ash which it contains.

1P. B. Morrison, As8't Professor of Animal Husbanary, University of wisconsin. 
TABLE I

CHELICAI ANAIYSIS OF FEEDS USED*

ITHED \% NITRO - \% CRUDE \% WATER \% FAT \% ASH \% FIEER \% N.F. E $\%$ CARBOGEN PROTEIN

\begin{tabular}{|c|c|c|c|c|c|c|c|c|c|}
\hline & ITAED & $\begin{array}{l}\text { \% NIIRO- } \\
\text { GEN }\end{array}$ & $\begin{array}{l}\text { \% CRUDE } \\
\text { PROTEIN }\end{array}$ & \% WATER & $\%$ IAT & $\%$ ASH & $\begin{array}{l}\text { \% FIBER } \\
\text { CRUDE }\end{array}$ & $\% \mathbb{N} \cdot \mathrm{F}, \mathrm{E}$ & $\begin{array}{l}\text { \% CARBO- } \\
\text { HYDRATES }\end{array}$ \\
\hline $\begin{array}{l}\text { Ro agh- } \\
8808\end{array}$ & $\begin{array}{l}\text { S1lage, alr ary } \\
\text { S1lag,, Iresh } \\
\text { T1mothy } \\
\text { Alfalfa }\end{array}$ & $\begin{array}{r}1.347 \\
.559 \\
.900 \\
2.296\end{array}$ & $\begin{array}{r}8.419 \\
3.494 \\
5.625 \\
14.350\end{array}$ & $\begin{array}{r}6.668 \\
61.290 \\
6.225 \\
6.065\end{array}$ & $\begin{array}{l}4.830 \\
2.003 \\
2.293 \\
2.350\end{array}$ & $\begin{array}{l}4.585 \\
1.902 \\
5.103 \\
8.715\end{array}$ & $\begin{array}{r}17.755 \\
7.364 \\
33.033 \\
33.658\end{array}$ & $\begin{array}{l}57 \cdot 743 \\
23 \cdot 947 \\
47 \cdot 721 \\
34.862\end{array}$ & $\begin{array}{l}75 \cdot 498 \\
31 \cdot 311 \\
80.754 \\
68.520\end{array}$ \\
\hline
\end{tabular}

* Department of Agricultaral Chemistry, Unlversity of Missourl The Agricul tural Chemistry Dopartment furnishod the analysis of the air dry material. The author made the caloulations for the composition of the fresh silage, protein, nitrogen free extract and carbohydrates. 
A comparison of the chemical analyses ${ }^{2}$ of the feeds used in this test with recent analyses of the same feods as reported by Henry and Lorrison, 1915 edition of Feeds and Feeding, shows the following: The silage fed was of excellent quality, being considerably lower in water content, somewhat higher in Piber and ash, and considerably higher in crude protein, nitrogen free extract and fat than the average of recent analyoes. The timothy hay was decidedly lower in water content, nitrogen free extract and slightly lower in crude protein and fat than the average for timothy as stated in Henry and Morrison Feods and Fooding. It was higher in piber and ash.

In comparison with the averages of recent analyses presented in the last edition of Henry and Morrison Feeds and Foeding, the alfalfa hay fed was lower in per cent of water, practicaliy the same in crude protein, ash and fat. It was higher in Plber and slightly lower in nitrogen free extract.

The corn fed was higher in water content, slightly higher in crude protein, lower in ash and fat, and was practically the same in fiber and nitrogen free extract as the average of recent analgses for corn.

A comparison of the oats used with the average of recent analyses for oats shows that they were practically the oame in percentage of water, ash, crude protein, higher in fiber and fat and slightly lower in nitrogen free extract 
The bran which was fed contained about the same amount of water, nitrogen free extract and fat as the average for recent analy8es of bran. It was slightly lower in ash and fiber and slightly higher in crude protein.

\section{TABIR II}

AVERAGE DIGESTIBILITY OF FEBDS ${ }^{2}$ - BXPERILIENTS WITH HORSES

FERD \& PROTEIN \& FIBER \& N.F.B\%TAT AUTHORITY

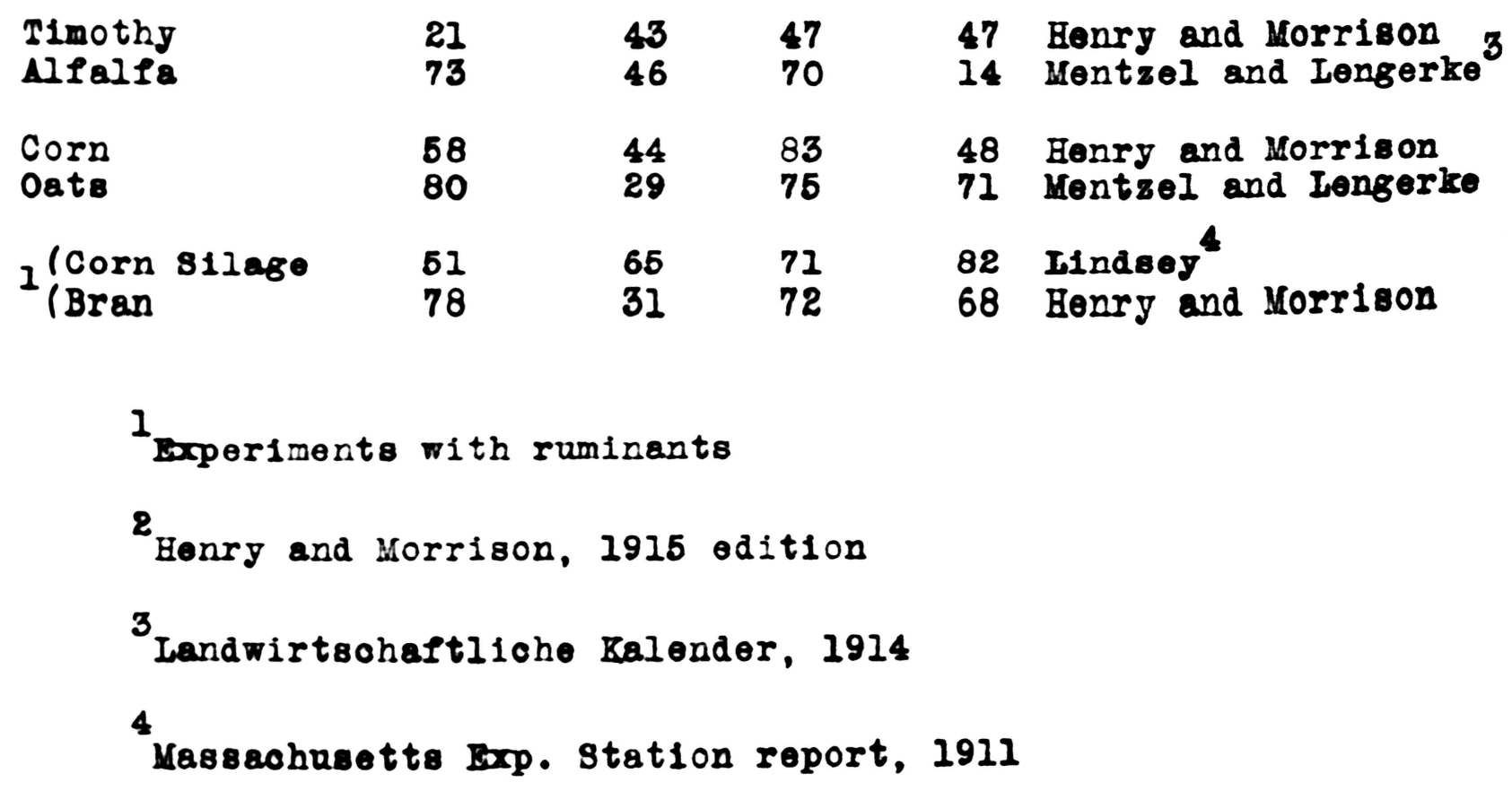

From rable II it will be noted that horses are able to digest more than eighty per cent of the nitrogen free extract in oorn. About one-half of the crude protein,fiber and fat in corn 18 digested by horser.

Horses digest a high peroentage of the nutrients in oats, exoept in the oase of the fiber.

The coefficient of digestibility of timothy hay for 
horses is Irom lorty-three to Iorty-seven for fiber, nitrogen free extract and fat, and twenty-one in the case of crude protein. Horses digest about seventy per cent of the crude protein and nitrogen free extract, and about fifty per cent of the fiber in alfalfa hay. Fourteen per cent of the fat in alfalfa hay is digested by horses. $"$ "The richer the feed, the more nearly do the digestive powers of the horse approach those of other animals. They digest less fiber than the ruminant in whose paunch the coarse foeds undergo special preparation and digestion."

\section{TABLE III}

DIGESTIBIE NUTRIENTS* IN 100 POUNDS OF FEEDS USED FEED DRY PROTEIN FIBER N. F. B. CARBO- FAT ASH

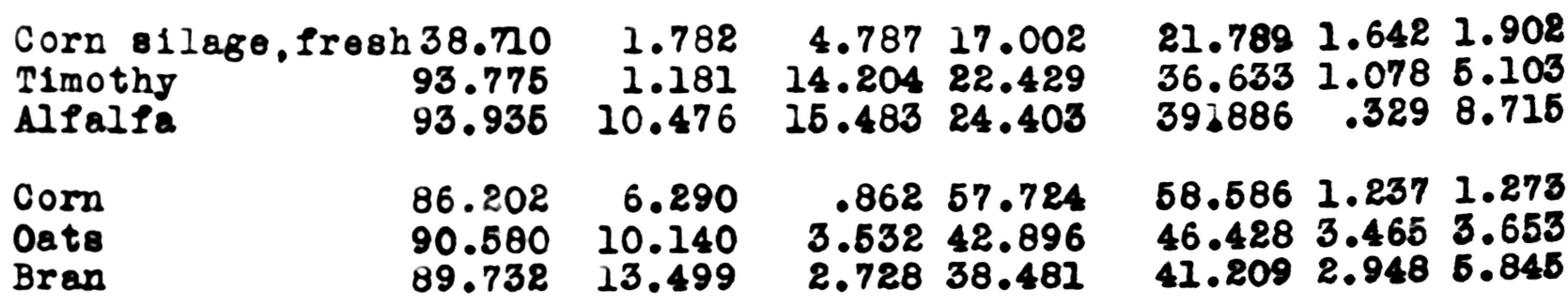
NUTRIENTS IN 100 POUNDS OF GRAIN FED, COMPOSED OF 2 POUNDS CORN, 2 POUNDS OATS AND I POUND BRAN BY WEIGHT Corn 2-0ats 2Bran 1 $88.659 \quad 9.272 \quad 2.303 \quad 47.944$ $50.247 \quad 2.4703 .135$

\footnotetext{
1 Henry and Morrison, p. 52, 1915 edition

*Pounds
} 
A comparison of the digestible nutrients in one hundred pounds of corn silage with those in one hundred pounds of timothy hay as seen in rable III shows that there is nearly two and one-half times as much dry matter in timothy hay as there is in silage. There is three tires as much digestible Iiber. slightly more digestible nitrogen ereo extract, more then one and one-half times as much digestible carbohydrates and more than two and one-half times as much ash, however, there is one and one-half times as much digestible protein and lat in one hundred pounds of silage as there is in the same amount of timothy haj.

In one hundred pounds of alfalfa hay there is two and one-half times as much dry matter, more than six times as much digestible protein, more than three times as much digestible fiber, more than one and one-half times as much digestible nitrogen freo extract, nearly twice as much digestible carbohydrates and five times as much ash as there is in the same amount of $\operatorname{corn} 8118 \mathrm{g \theta}$.

The oorn sllage contained more than five times as much digestible fat. 
DESCRIPIION OF HORSES IN THE 1914-1915 TEST

IOT I

IOT II

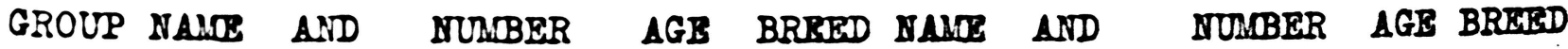

\begin{tabular}{|c|c|c|c|c|c|c|c|}
\hline $\begin{array}{r}A-0 \\
B-a \\
b\end{array}$ & $\begin{array}{l}\text { Ruth MoDonela } \\
\text { Julo } \\
\text { Waok }\end{array}$ & $\begin{array}{l}110.11 \\
110.9 \\
110.7\end{array}$ & $\begin{array}{r}10 \\
13 \\
8\end{array}$ & $\begin{array}{l}\text { Ilght } \\
\text { hrale } \\
\text { Grade }\end{array}$ & $\begin{array}{l}\text { Marthe 2nd } \\
\text { Kate } \\
\text { Cap }\end{array}$ & $\begin{array}{l}110.12 \\
10.10 \\
10.8\end{array}$ & $\begin{array}{r}12 \\
16 \\
8\end{array}$ \\
\hline $\begin{array}{l}0 \\
0 \\
-b-7 \\
0\end{array}$ & 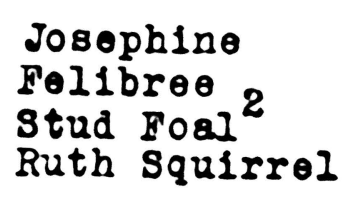 & $\begin{array}{l}\text { N10.1 } \\
\text { N10.3 } \\
\text { No.19 } \\
\text { No.17 }\end{array}$ & $\begin{array}{c}12 \\
3 \\
\text { rogl }\end{array}$ & $\begin{array}{l}\text { DraIt } \\
\text { Draft } \\
\text { Draft } \\
\text { Draft } \\
\text { Iight }\end{array}$ & $\begin{array}{l}\text { Porgette } \\
\text { Pinette- } \\
\text { Plily Forl } \\
\text { Hegne of }\end{array}$ & $\begin{array}{l}110.2 \\
110.44 \\
110.20 \\
110.18\end{array}$ & $\begin{array}{r}7 \\
6 \\
\text { Pogl }\end{array}$ \\
\hline$a-2$ & Pepper & No.15 & 2 & Ifght & $\begin{array}{l}\text { Iady } \\
\text { Adrance }\end{array}$ & 80.16 & \\
\hline$b$ & $\begin{array}{l}\text { Josephe } \\
\text { Ione Rex }\end{array}$ & $\begin{array}{l}\text { No. } 5 \\
\text { HO. } 33\end{array}$ & $\begin{array}{l}2 \\
3\end{array}$ & $\begin{array}{l}\text { Dre } \\
\text { If }\end{array}$ & $\begin{array}{l}\text { Mona } \\
\text { Satelite }\end{array}$ & $\begin{array}{l}\text { No. } 6 \\
\text { No.14 }\end{array}$ & $\varepsilon$ \\
\hline
\end{tabular}

\section{MANAGEMTENT DURING SUREER AND FALI PRE-}

VIOUS TO THE BEGIMTING OF THE TEST

Horses numbers 1, 2, 4, 7, 8 and mules numbers 9 and 10 were on dry feed and were worked during the summer and fall. Numbers 3 and 5 were on pasture until julg and number 6 was on pasture unt1l ootober 18t. They were put on dry feed and worked when they were brought in from pasture. The other horses were not worked.

Numbers 12, 19 and 20 rere on dry feed during the axmmer and fall. Numbers 11 and 17 were on pasture until Soptember 1st, and numbers 13,14, 15, 16 and 18 were on pasture until ootober 30th when they were put on dry leed.

\footnotetext{
I Proved later not in forl

2 Dam, Pinette, Sire, Gardiner 3 Dam, Josephine, Sire, Gardiner
} 
FBED RECEIVED

Tables IV, IV, VI, VII, VIII, IX, X, XI and XII give the quantity of feed consumed, work performed and weights of each individual by weokly periods. In addition the total and the average daily consumption of leed are shown. These data are presented for weekly periods in order that variations in the amount of feed consumed and in weight may be noted.

The "ollage fed horses" did not consume large quantitios of the silage the first week. They ate from one pound daily in the case of draft foal Number 19 to 7.57 pounds per day in the 0 ase of the afed saddle mare, Ruth MoDonald No. Il. The hay and in most cases the grain was reduced at the end of the first week, after which larger quantities of silage were consumed.

At the close of the experiment the horses were eating from 6 pounds daily in the case of saddle forl, Ruth squirrel No. 17 to 12.39 pounds in c8se of the "in fosl" draft brood mare, Pelibreo No. 3 .

It was natural that small quantities of silase were consumed at first because the horses had all been on dry feed for some time and none of them had erer been fed silage before. The average of the "silage fed horses" ate less 81lage the last weok of the test than they had previously consumed. The silage fed the last week was of poor quality. It contained a high per cent of water and acid, being near the 
bottom of the 8110 .

DIGBSTIBIR NUTRIENTS RECEIVED

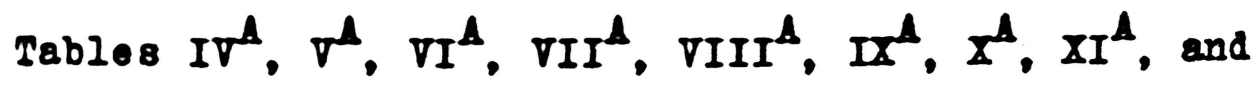
$X I I^{A}$, show the total digestible nutrients receired and the average digestible nutrients led daily. They also present the totel digestible nutrients consumed and the average digestible nutrients recelred dally per 1,000 pounds live weight of the horses.

This was based on the average weight of each horse thru the test. 
TABLE IV

Group \&

MATURR IDIE HORSES AND MULES

o. Pregnant Mares

Lot I. Ruth MoDonald, Ho. 11, Registered Saddlo Maro

Lot II. Karthe 2nd, No. 12, Registered Saddlo Maro

\section{PEED CONSUAED* AND WEIGHT* BY WEEKLY PERIODS}

IOT I

\begin{tabular}{|c|c|c|c|c|c|c|c|}
\hline $\begin{array}{c}\text { DATE } \\
1914 \\
\text { Deo. } 24 \\
N\end{array}$ & $I_{\text {GRAIN }}$ & $Z_{\text {HAY }}$ & SIIUAGE & $\begin{array}{l}\text { WEIGET } \\
3 \begin{array}{l}1195 \\
1170\end{array}\end{array}$ & ${ }^{1}$ GRAIN & $2_{H \triangle Y}$ & $\begin{array}{c}\text { WEIGHT } \\
3_{1200} \\
1180\end{array}$ \\
\hline \begin{tabular}{cc}
\multicolumn{2}{c}{1915} \\
Jan. & 7 \\
$n$ & 14 \\
$n$ & 21 \\
$"$ & 28 \\
Iob. & 4 \\
$n$ & 11 \\
$n$ & 18 \\
$n$ & 25 \\
March & 4 \\
" & 11 \\
" & 18
\end{tabular} & $\begin{array}{l}28 \\
28 \\
28 \\
28 \\
28 \\
35 \\
35 \\
35 \\
35 \\
35 \\
35\end{array}$ & $\begin{array}{l}66 \\
56 \\
56 \\
56 \\
56 \\
70 \\
70 \\
70 \\
70 \\
70 \\
70\end{array}$ & $\begin{array}{l}60 \\
74.25 \\
80.5 \\
88 \\
83.5 \\
83 \\
81.75 \\
83.5 \\
80.75 \\
81 \\
76\end{array}$ & $\begin{array}{l}1205 \\
1225 \\
1250 \\
1270 \\
1270 \\
1290 \\
1280 \\
1315 \\
1315 \\
1330 \\
1308\end{array}$ & $\begin{array}{l}28 \\
28 \\
28 \\
28 \\
28 \\
28 \\
28 \\
28 \\
28 \\
28 \\
28\end{array}$ & $\begin{array}{l}83.5 \\
70 \\
68 \\
69.75 \\
69 \\
69.75 \\
65.75 \\
70 \\
70 \\
70 \\
70\end{array}$ & $\begin{array}{l}1195 \\
1205 \\
1220 \\
1235 \\
1230 \\
1250 \\
1220 \\
1210 \\
1250 \\
1265 \\
1245\end{array}$ \\
\hline tal & 378 & 780 & 919.25 & ${ }^{4} 113$ & 332 & 855.75 & 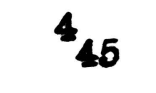 \\
\hline Da1lg Av. & 4.5 & & 10.943 & & 3.95 & 10.19 & \\
\hline
\end{tabular}

\section{*Pounds}

ICorn, 0ats, Bran, 2 parts - 2 parts - 1 part, by weight 2 Nlalfa hag

3

Intigl woight

4

Gain in woight 


\section{TABLE IN}

LOT I

DIGESTIBLE NUTRIBNTS CONSURED*

\begin{tabular}{|c|c|c|c|c|c|c|}
\hline & $\begin{array}{l}\text { TOTAL FEED } \\
\text { COHSUIRED }\end{array}$ & DRY MATIER & PROTBIN & $\begin{array}{l}\text { CARBO- } \\
\text { HYDRATES }\end{array}$ & PAT & ASH \\
\hline $\begin{array}{l}\mathcal{Z}_{\text {Gay }} \\
\text { S1Iage }\end{array}$ & $\begin{array}{l}378.0 \\
780.0 \\
919.26\end{array}$ & $\begin{array}{l}335.131 \\
732.693 \\
355.848\end{array}$ & $\begin{array}{l}35.048 \\
81.713 \\
16.381\end{array}$ & $\begin{array}{l}189.932 \\
311.110 \\
200.295\end{array}$ & $\begin{array}{r}9.337 \\
\mathbf{2 . 5 6 6} \\
16.094\end{array}$ & $\begin{array}{l}11.850 \\
67.977 \\
17.484\end{array}$ \\
\hline Total & & 1423.666 & 133.142 & 701.337 & 27.997 & 97.311 \\
\hline Av. Dully & & 16.948 & 1.585 & 8.349 & .333 & 1.158 \\
\hline
\end{tabular}

DIGBSTIBLB NUTRIENTS CONSUMAD*

PIER 1,000 POUNDS LIVS WEIGHT

Total

Ar. Daily

1145.301

107.109664 .207

22.526

78.284

13.635

$1.275 \quad 6.717$

$.268 \quad .938$

\section{IOT II}

DIGBSTIBLE NUTRIENTS CONSONGE*

Z Orain $_{\text {Hay }}^{1}$

Total

Ar. Daily

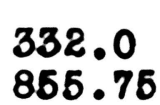

855.75

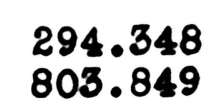

1098.197

13.074
30.783166 .820

89.648341 .385

120.431508 .145

$1.434 \quad 6.049$
$8.20 \quad 10.408$

$8.815 \quad 74.579$

$11.015 \quad 84.987$

$.131 \quad 1.012$

DIGESTIBIE IUTRIENTS CONSUMED*

PER 1,000 POUNDS LIVS WEIGHT

Total

Av. Dailg

899.679

98.650416 .243

$9.083 \quad 69.616$

10.709

$1.174 \quad 4.955$

.107 .889

ICorn, Oats, Bran, 2 parts - two parts - I part infealfa hay

*Pound 8 


\section{GROUP A - C}

PHOTOGRAPHED AT THE CLOSE OF THE BXPERIMENT, MARCH 15, 1915 LOT II MARTHA 2ND, NUMBER 12-LOT I RUTH MODONALD, NUMBER 11

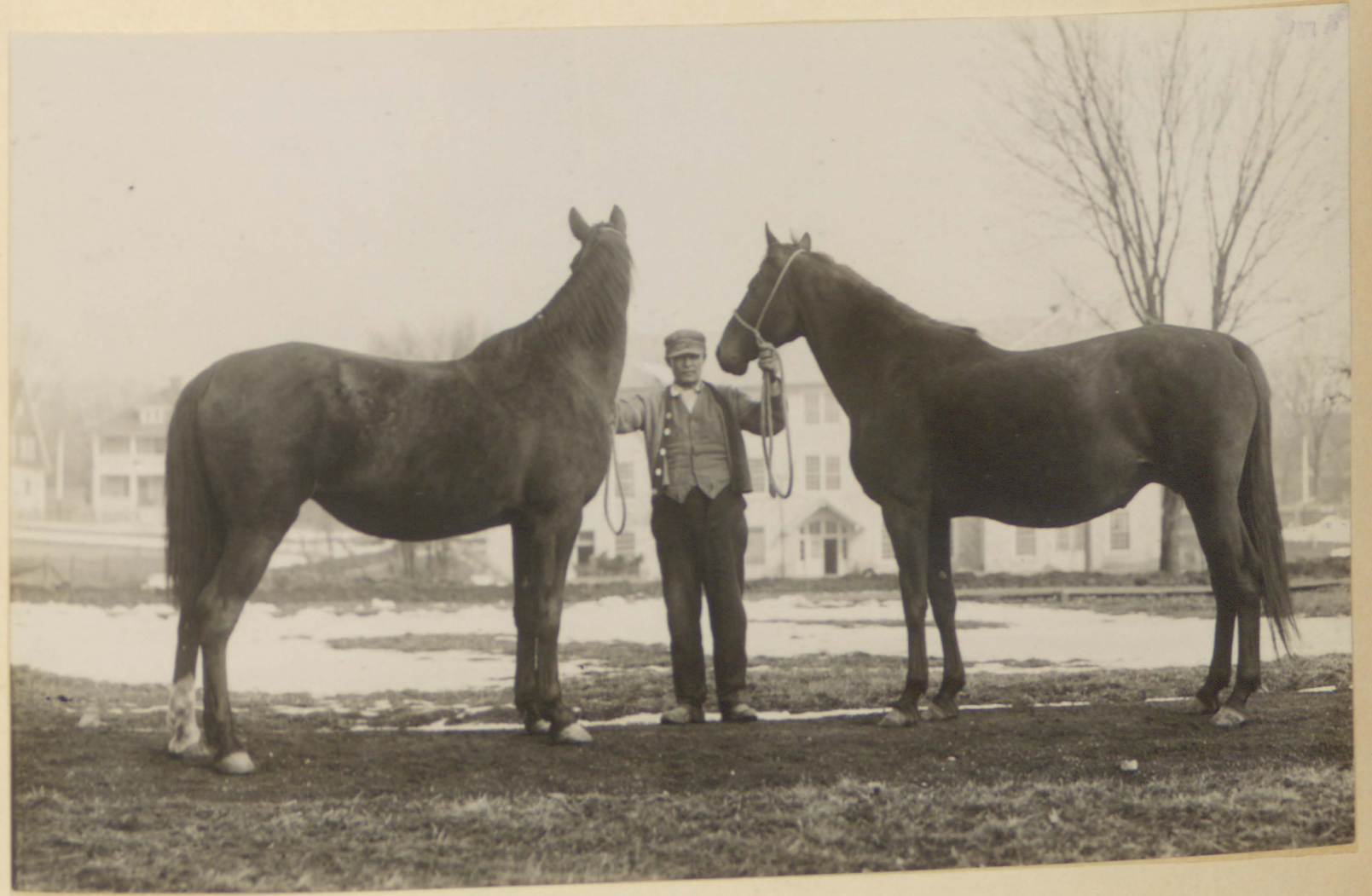

MATURB IDLE PREGNANT MARES

NO SILAGE

SIIAGE 
Tables IV and IV present the data for Group $A-c$, Lots I and II, mature idie pregnant mares, Ruth MoDonald, No. 11 and Narthe 2nd, No. 12. Lot I receired silage as a part ration. Lot II was fod no silage. Both lots receired grain and alfalfa hay.

These mares were nearly equal in weight and both were in medium condition of fiesh at the beginning of the experiment. Mare No. 11 was nearly six weoks further advanced in period of gestation than Mare No. 12. Mare No. 11 was bred April 2I, 1914 and Mare No. 12 was bred June 10, 1914. Ruth MoDonald, No. 11, produced a foal in the spring of 1914 and suckled this foal until the first of october, about six weeks before this test began. The other mare was not pregnant the previous year.

Lot I gained 68 pounds more than Lot II during the trial. Lot I consumed .55 pounds more grain and .92 pounds 1088 hay dally. In addition to the grain and hay received, Lot I was fed an average of 10.94 pounds of corn silage daily. Lot II was led grain and hay.

The table of digestible nutrients consumed shows that more No. 11 received 3.874 pounds of dry matter, slightly more protein and more than one and one-half times as much carbohydrates dally than was consumed by mare No. 12. Mare No. Il was fed more than twioe as much fat and slightly more ash daily than was fed to mare No. 12.

These facts hold true when reduced to a thousand pound basis for the reason that the lots did not differ greatly 
in woights.

Lt the close of the test the sllage fed mare was in better condition than mare No. 12. Her hair was in excollent condition, being short and glos8y. Neither mare was fat. These mares were in good thrifty breeding condition at the close of the test.

since this was an initial effort in feeding silage to brood mares at this experiment otation, Lot I was allowed to consume more nutrients than Lot II. 


\section{TABIE V \\ GROUP B}

LATURE HORSES AND MULES DOING AVERAGE WINTER FARM WORK

a. Lales

Lot I. Jule, No. 9

Lot II. Kate, No. 10

FEED CONSUUED; WEIGHTS*AND TORK HOURS BY WEEKCY PERIODS

\section{IOT I}

DATE ${ }^{1}$ GRAIN ${ }^{2}$ HAY SILAGE WEIGHT ${ }^{3}$ TORR 1914

Dec.24

Dec.3I 1915 $\operatorname{Jan} .14 \quad 47.54 .5$ Jan.21 51. 55.5 Jan.28 5I. 56 . Fob. 4 42. 56 . Fob.11 45. 56. Fob.18 45. 56 . Fob.25 $35.5 \quad 53.5$ Moh. 439.556. Moh.11 42. 56. Koh.18 42. 56 . Total $522.5682 \quad 676$ $\begin{array}{rrr}4 & \text { HOURS } \\ 1460 & 1 & B \\ 1440 & 3\end{array}$ $12.0 \quad 1440$

\section{IOT II}

$1_{\text {GRAIN }}{ }^{2}$ HAY TRIGHT ${ }^{3}$ TORK $\begin{array}{llllll}B & & & 4 & & \text { HOURS } \\ 3195 & 4 & B \\ 3 & 40.25 & 80.75 & 1195 & & 3\end{array}$ $\begin{array}{lllllll}45.5 & 1455 & 3 & 41 . & 83.75 & 1175 & 3\end{array}$ 65. $\quad 1455 \quad 10 \quad 34 \quad 62$. $75.25 \quad 1450 \quad 3268$. $64.75 \quad 1445 \quad 10 \quad 25$ 79 . 1470 68.51460 51. 1410 56. 1390 $53.75 \quad 1410$ 75. 1435 80.251415 $5_{45 \quad 28 \quad 142 \quad 652.25 \quad 1034}$ 456.95 .5 25 54. 96. 56. $74.75 \quad 1180$

7 52. 85. 1170 $\begin{array}{llll}81 . & 1190 & 10 & 34 \\ 94 . & 1175 & & 32\end{array}$ $\begin{array}{llll}94.5 & 1190 & 10 & 25\end{array}$ 
TABLE $\nabla^{\text {A }}$

IOT I

DIGBSTIBLE NUMRIEMTS CONSUNED*

\begin{tabular}{|c|c|c|c|c|c|c|}
\hline & $\begin{array}{l}\text { TOTAL FEED } \\
\text { CONSUIED }\end{array}$ & DRY MATTRR & PROTEIN & $\begin{array}{l}\text { CARBO- } \\
\text { EYDRATES }\end{array}$ & FAT & ASH \\
\hline $\begin{array}{l}2_{\text {Grain }} \\
\text { Bay } \\
\text { S1lage }\end{array}$ & $\begin{array}{l}522.50 \\
682.0 \\
675.0\end{array}$ & $\begin{array}{l}463.243 \\
639.546 \\
261.293\end{array}$ & $\begin{array}{r}48.446 \\
8.054 \\
12.029\end{array}$ & $\begin{array}{l}262.541 \\
249.837 \\
147.074\end{array}$ & $\begin{array}{r}18.906 \\
7.352 \\
11.084\end{array}$ & $\begin{array}{l}16.380 \\
34.802 \\
12.839\end{array}$ \\
\hline Total & & 1364.082 & 68.529 & 659.452 & 31.342 & 64.021 \\
\hline$\Delta \nabla$. & & 16.239 & .816 & 7.851 & .373 & .762 \\
\hline
\end{tabular}

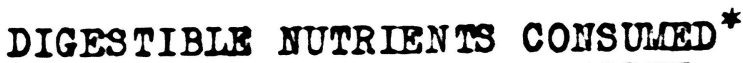

PER 1,000 POUIDS IIVB WEIGET

Total

Av. Da11y
947.799

11.283
47.616

.567
465.158

5.538
21.77744 .481 $.259 \quad .530$

\section{IOT II}

DIGESTIBLE NUTRIENTS CONSURED*

$\begin{array}{lrrrrrr}\text { I Grain }_{\text {Gaj }} & 652.25 & 588.278 & 60.477 & 327.736 & 16.111 & 20.448 \\ \text { Total } & 1034.0 & 939.634 & 12.212 & 378.785 & 11.146 & 52.765 \\ & & 1557.912 & 72.689 & 706.521 & 27.257 & 73.213 \\ \text { Ar. Dally } & & 18.547 & .865 & 8.411 & .324 & .872\end{array}$

DIGESTIBIS NUTRIEITS CONSURED*

PER 1,000 POUNDS IIVB WEIGET

$\begin{array}{lrrrrr}\text { Total } & 1308.486 & 61.051 & 510.252 & 22.893 & 61.491 \\ \text { Lr. Da1ly } & 15.577 & .726 & 6.074 & .273 & .732\end{array}$

${ }^{1}$ corn, oats, bran, 2 parts - 2 parts - I part, by weight

8

Timothy hay

*Pound 8 


\section{GROUP B - a}

PHOTOGRAPHED AT THE CLOSE OF THE EXPERIMENT, MARCH 15, 1915 LOT II KATE, NUMBER 10 IOT I JULE, NUMBER 9

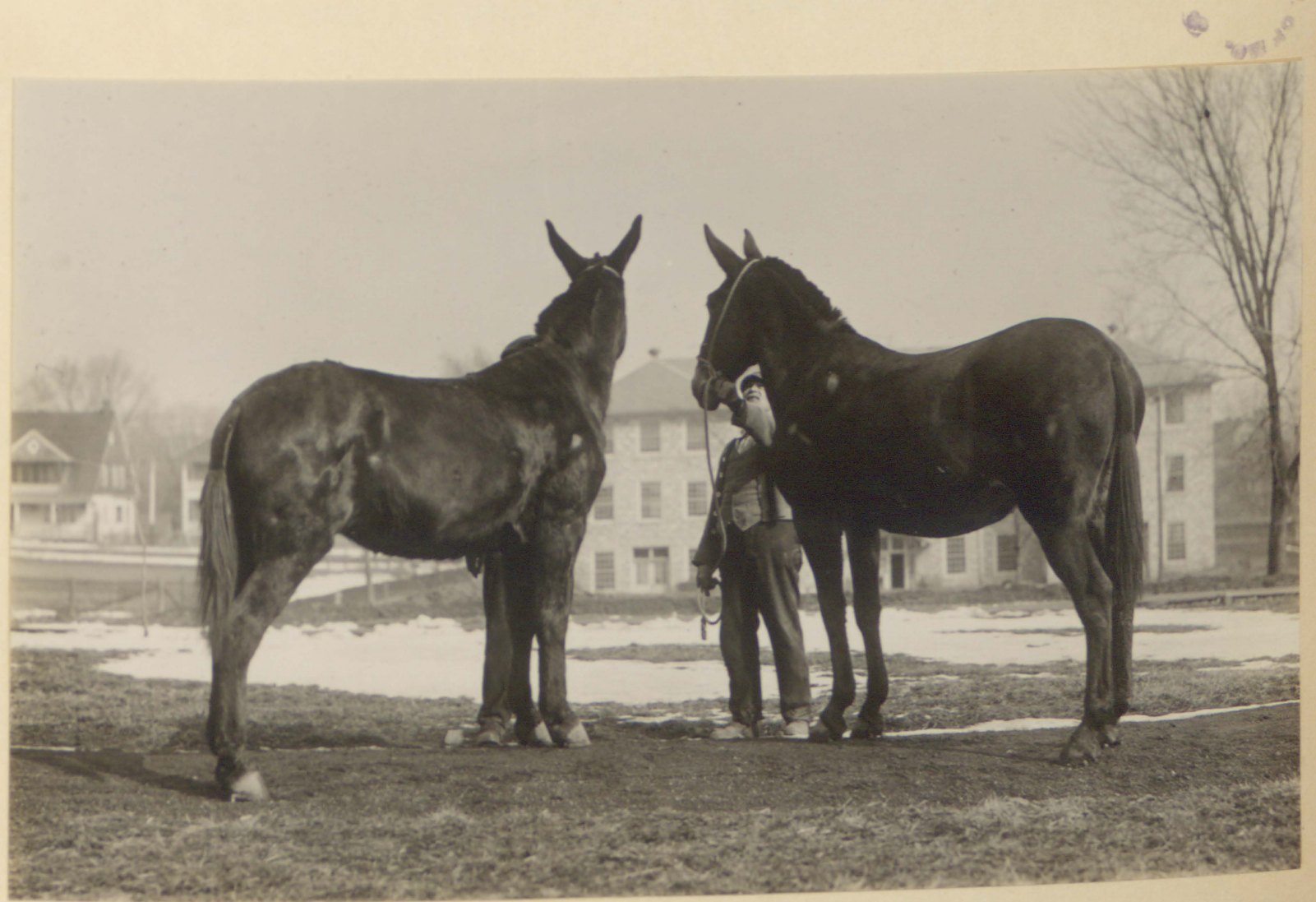

MATURE MULES PERFORMING AVERAGE WINMER FARM WORK NO SILAGE 
Tables $V$ and $\nabla^{A}$ present the data for Group B-a, mules, Jule No. 9 and Kate No. 10, performing average winter farm work.

Lot I was fed silage as a part ration. Lot II received no silage. Both lots were fed grain and timothy hay. This was the only team of mules fed during either test. The silage fed mule was considerably larger than her mate as is shown by the initial weights. The initial weight of mule No. 9 was 1460 pounds while the initial weight of Mule No. 10 was 1195 pounds. This team was in good working condition at the beginning of the test. Mule No. 9 was in slightly better condition than mule No. 10.

These mules performed 28 hours of heavy work and 142 hours of medium work, making an aggregate of 170 hours of work during the test. They worked an average of 2.36 hours per working day.

Lot I received an average of 1.55 pounds of grain and 4.19 pounds of hay per day less than Lot II. Lot I consumed in addition to the grain and hay fed an average of 8.05 pounds of silage daily.

It will be noted from the table $v^{M}$, digestible nutrients consumed, that Lot II received 2.308 pounds of dry matter, .049 pounds of protein, .56 pounds of carbohydrates, .11 pounds of esh more per day than Lot I. Lot I received .049 pounds of lat daily more than Lot II.

When reduced to a thousand pound basis table $v^{A}$ shows 
that Lot II received an average daily excess over Lot I of 4.294 pounds of dry matter, .159 pounds of protein, .536 pounds of carbohydrates, .014 pounds of fat and .202 pounds of ash.

Lot I lost 45 pounds and Lot II lost 5 pounds in weight during the test. At the elose of the experiment Iot I, the "81lage fed mule," was in much better thrift than lot II. The hair and skin of the male that was fed silage was in excellent condition. while the hair and skin of the other mule was in only modium condition. 


\section{TABLE VI}

GROUP B

MATURE HORSES AND MULES DOING AVERAGE TINTER PARM TORK

b. Goldings and open mares

Lot I. Mack, No. 7, grade draft gelding

Iot II. Cap, No. 8, grade draft gelding

FRED CONSULRD, ITIGHMS*AND WOP.K HOURS BY WEEKCYY PERIODS

$$
\text { LOT I LOT II }
$$

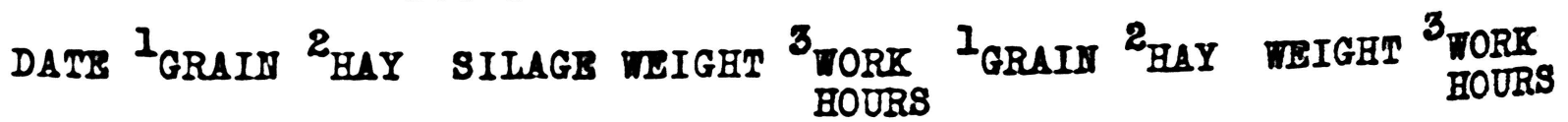

\begin{tabular}{|c|c|c|c|c|c|c|c|c|c|c|}
\hline $\begin{array}{r}1914 \\
0.24 \\
31\end{array}$ & 72.5 & 75. & 10. & $\begin{array}{r}4366 \\
1340\end{array}$ & & $\begin{array}{c}B \\
41.0\end{array}$ & 100. & 139. & $\begin{array}{r}{ }^{4} 1336 \\
1360\end{array}$ & $A_{41: 0}^{B}$ \\
\hline $\begin{array}{r}7 \\
14 \\
21 \\
28 \\
\text { b. } 4 \\
11 \\
18 \\
25\end{array}$ & $\begin{array}{l}59 . \\
80 . \\
91 . \\
100 . \\
100 . \\
100 . \\
100 . \\
100 . \\
100 . \\
100 . \\
100 .\end{array}$ & $\begin{array}{l}53.5 \\
65.75 \\
64 . \\
70 . \\
70 . \\
70 . \\
70 . \\
84 . \\
84 . \\
84 . \\
84 .\end{array}$ & $\begin{array}{l}66.75 \\
78 . \\
84.25 \\
93.75 \\
97.5 \\
90 . \\
95 . \\
91.5 \\
63.5 \\
88 . \\
78 .\end{array}$ & $\begin{array}{l}1340 \\
1365 \\
1340 \\
1390 \\
1350 \\
1350 \\
1340 \\
1330 \\
1330 \\
1335 \\
1329\end{array}$ & $\begin{array}{l}10 \\
30 \\
10\end{array}$ & $\begin{array}{l}28.0 \\
57.5 \\
25.0 \\
44.0 \\
43.5 \\
54.0 \\
55.0 \\
46.0 \\
50.0 \\
27.0 \\
50.0\end{array}$ & $\begin{array}{l}100 . \\
100 . \\
100 . \\
100 . \\
100 . \\
100 . \\
100 . \\
100 . \\
100 . \\
100 . \\
100 .\end{array}$ & $\begin{array}{l}132.5 \\
110.5 \\
105 . \\
105 . \\
105 . \\
105 . \\
105 . \\
105 . \\
111 . \\
119 . \\
119 .\end{array}$ & $\begin{array}{l}1325 \\
1340 \\
1325 \\
1320 \\
1300 \\
1325 \\
1310 \\
1280 \\
1280 \\
1300 \\
1292\end{array}$ & $\begin{array}{rr}10 & 28.0 \\
& 57.5 \\
30 & 25 . \\
10 & 44.0 \\
& 43.5 \\
54.0 & 5 \\
55.0 & \\
& 46.0 \\
10 & 50.0 \\
15 & 27.0 \\
10 & 50.0\end{array}$ \\
\hline & 1102.5 & 874.25 & 930.25 & ${ }^{5} 37$ & 85 & 521 & 1800 & 1361 & $5_{54}$ & 85521 \\
\hline & 13.18 & 10.4 & 11.07 & & & & 14.28 & 816.8 & & \\
\hline
\end{tabular}

${ }^{1}$ corn, oats, bran, $2-2-1$

8

Timothy has

$3_{A}$ - heary work, B - medium and light work

Inftial weight

5

L088 In weight 


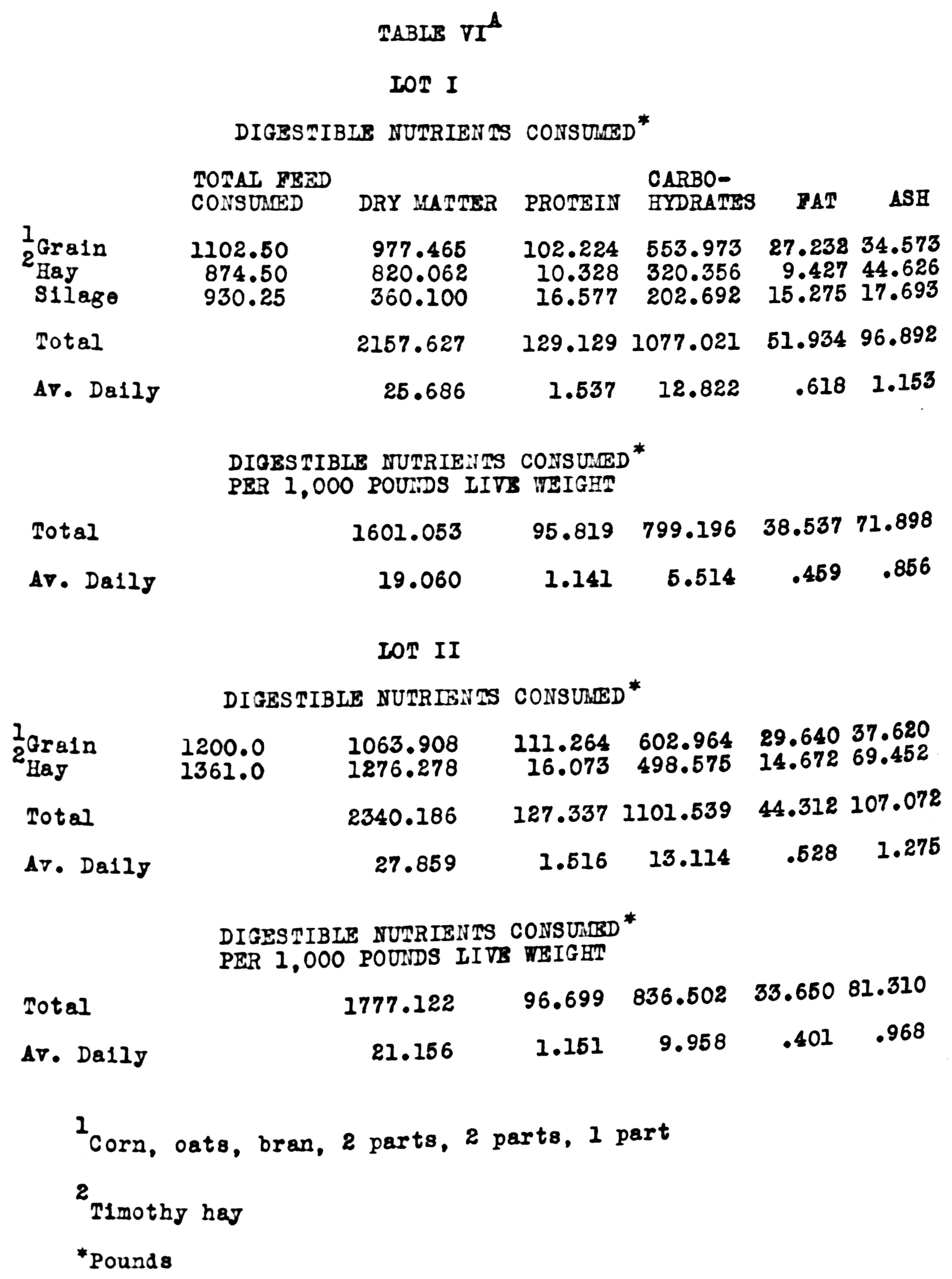

\begin{tabular}{|c|c|c|c|c|c|c|}
\hline$\sum_{\text {Hay }}^{1}$ & $\begin{array}{l}1200.0 \\
1361.0\end{array}$ & $\begin{array}{l}1063.908 \\
1276.278\end{array}$ & $\begin{array}{r}111.264 \\
16.073\end{array}$ & $\begin{array}{l}602.964 \\
498.575\end{array}$ & $\begin{array}{l}29.640 \\
14.672\end{array}$ & $\begin{array}{l}37.620 \\
69.452\end{array}$ \\
\hline Total & & 2340.186 & 127.337 & 1101.539 & 44.312 & 107.0 \\
\hline Da1ly & & 27.859 & 1.516 & 13.114 & .528 & 1.275 \\
\hline
\end{tabular}

DIGESTIBIE NUTRIENTS CONSUIIBD*

PER 1,000 POUNDS IIVB WEIGET

Total

1777.122

$96.699 \quad 836.502 \quad 33.650 \quad 81.310$

Av. Da1Iy

21.156

1.151

9.958 $.401 \quad .968$

$1_{\text {Corn, oats, bran, } 2 \text { parts, } 2 \text { parts, } 1 \text { part }}$

2

Timothy has

*Pound 8 


\section{GROUP B - b}

PHOTOGRAPHED AT THE CLOSE OF THE FXPERIMENT, MARCH 15,1915 LOT II CAP, NUMBER 8 LOT I MACK, MUMBER 7

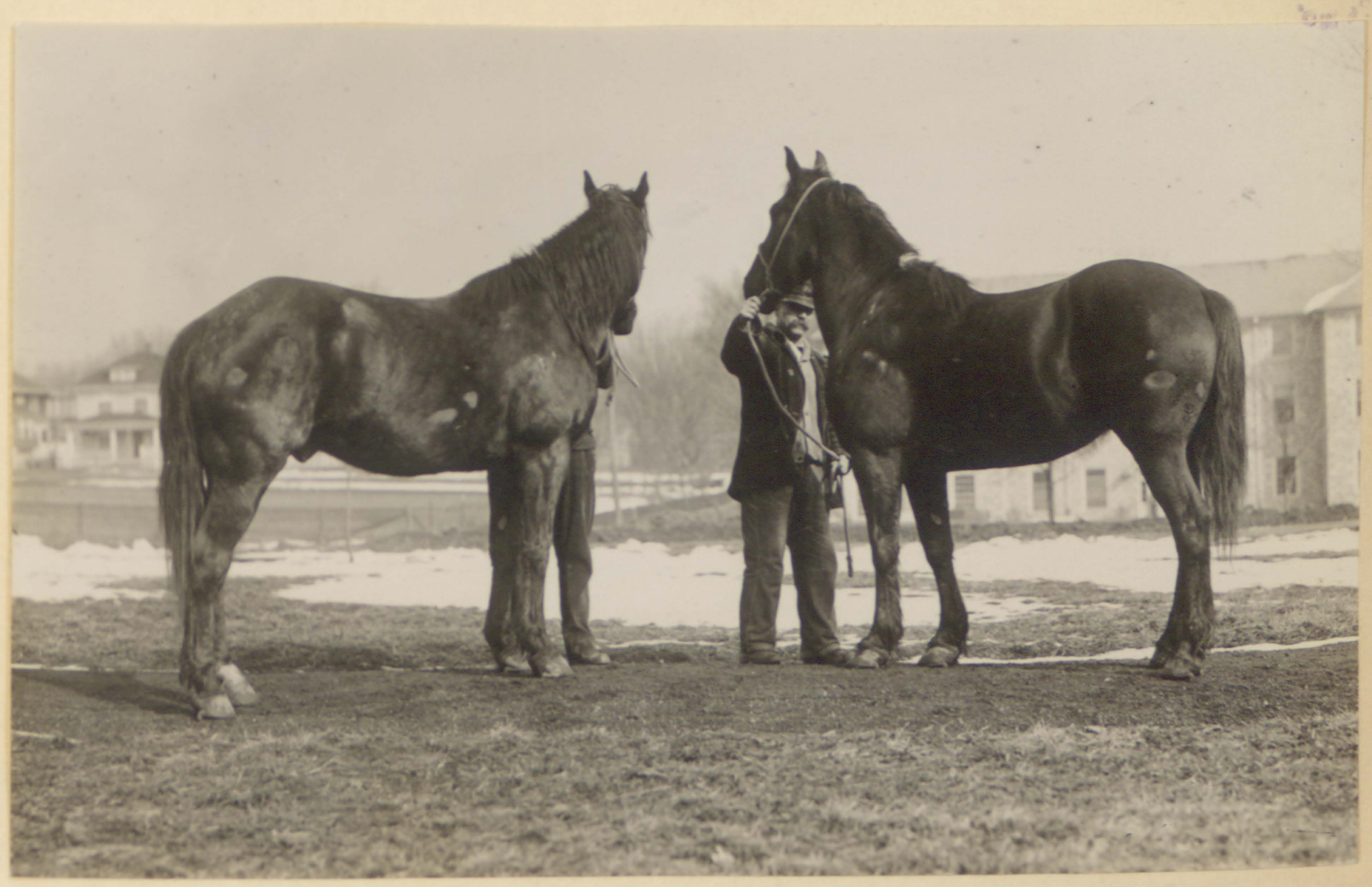

MATURE GRIDINGS PERFORMING AVERAGE WINTMR FARM WORK NO SILAGE SIIAGE 
Tables VI and VI present the data for Group B-b, grade draft geldings, Mack, No. 7 and Cap, No, 8, performing average winter farm work.

Lot I was fed silage as a part ration. Lot II recolred no silage. Both lots were fed grain and timothy haj. These geldings were in good working condition at the beginning of the test. There was no noticeable difference in their condition. The initial weight of gelding No. 7 was 30 pounds moro than the inftial weight of gelding No. 8. Gelding No. 7 was a olightly larger framed horse than gelding No. 8, which accounts for the deference in thoir initial weights.

During tine test this team of geldings performed 606 hours of work, 85 hours was classed as heary work and 521 hours was medium or light work. They performed an average of 8.42 hours labor per working daj.

Lot II consumed a daily average of 1.12 pounds of grain and 5.8 pounds of hag more than Lot $I$. Lot I receired an arerage of 11.07 pounds of silage daily.

Table VI shows that Lot II received an arerage of 2.173 pounds dry matter, .292 pounds carbohydrates and .122 pounds of ash more per day than Lot I. Lot I received an average of .021 pounds of protein and .090 pounds of fat more than Iot II. Then reduced to a thousand pound basis this table shows that Lot II consumed an average per day of 2.096 pounds dry matter, .010 pounds protein and .444 pounds carbohydrates, also .112 pounds of ash more than Lot I. Lot I was fed .058 pounds lat per day more than Iot II. 
This team lost in weight during the test. Gelding No. 7 lost 37 pounds and gelding No. 8 lost 54 pounds. They had worked hard during the trial.

At the close of the experiment both horses were in good thrifty working condition. They were not fat. Gelding No. 7 was in slightly better condition of flesh and his coat of hair indicated better thrift than that of gelding No. 8 . 


\section{TABIE VII}

GROUP B

MUTURE BORSES AND LUTLES DOING AVERAGE WINTER FAPM TORK

c. - I Pregnant mares

Lot I. Josephine, Ho. 1, Registered Percheron mare

Lot II. Porgette, No. 2, Registered Percheron mare

FBED CONSULRD, WEIGEMS* AND WORK HOURS BY WEEKLY PERIODS

$$
\text { IOT I }
$$

IOT II

DATE $1_{\text {GRAIN }} 2_{\text {HAY }}$ SILAGE WBIGHT ${ }^{3}$ TORK ${ }^{1}$ GRAIN $2_{\text {HAY WEIGHT }} 3_{\text {TORK }}$

1914

Dec.24

n 3187

1915

Jan. 766

" 1491

" 21100

n 28107.5

Feb. 4110.

n 11 120. 98

n 18 110. 98

n 25 110. 98

Noh. 4110 . 96.5

n 11110.98

$\begin{array}{lllll}60 & 52.5 & 1540 & 27 & 21\end{array}$

$A B$

$4_{1637}$

A B

n $1893.5 \quad 98 \quad 61.25 \quad 1653$

$60.75 \quad 1560 \quad 25 \quad 13$

$\begin{array}{lllll}120 & 111.75 & 1625 & 27 & 21\end{array}$

$46 \quad 60.75 \quad 1560$

$\begin{array}{lllll}120 & 110.75 & 1678 \quad 25 & 13\end{array}$

56 57. 1590

42. 1595

$38.5 \quad 1563$

76

61. $\quad 1560$

$61.25 \quad 1595$

61. 1620

64. 1600

68.251620

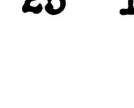

109103.501700

$5 \quad 100 \quad 111.501730$

$45 \quad 100 \quad 111.501660$

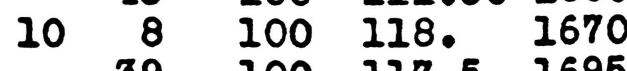

$100 \quad 117.5 \quad 1695$

$\begin{array}{lllll}10 & 41 & 100 & 118.5 & 1630\end{array}$

$100 \quad 118.5 \quad 1640$

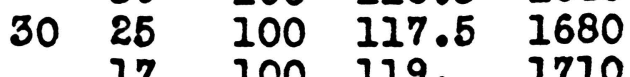

100 119.5 1710

${ }^{5} 81 \quad 102 \quad 253 \quad 1234 \quad 1373.5$

$574 \quad 202 \quad 253$

Total $1205 \quad 964.5695 .5$

Daily

ATe.

$\begin{array}{lll}14.34 & 11.48 & 8.28\end{array}$

$14.69 \quad 16.35$

${ }^{*}$ Pound 8

1

Corn, oats, bran, 2-2-1

I Timothy hay

$3_{A}$ - heary work, B - medium and light work

4 Initial woight

${ }^{\text {Gain in woight }}$ 


\section{TABLB VII \\ IOT I}

DIGESTIBLE NUMRIEN TS CONSUNED*

\begin{tabular}{|c|c|c|c|c|c|c|}
\hline & $\begin{array}{l}\text { TOTAL FHED } \\
\text { CONSURED }\end{array}$ & DRY KATTER & PROTEIN & $\begin{array}{l}\text { CARBO- } \\
\text { HYDRATES }\end{array}$ & FAT & ASH \\
\hline $\begin{array}{l}{ }_{2}^{1} \text { Grain } \\
\text { Haj } \\
\text { S1lage }\end{array}$ & $\begin{array}{c}1205.0 \\
964.50 \\
695.5\end{array}$ & $\begin{array}{r}1068.341 \\
904.460 \\
269.228\end{array}$ & $\begin{array}{r}111.728 \\
11.391 \\
12.394\end{array}$ & $\begin{array}{l}605.476 \\
353.326 \\
151.553\end{array}$ & $\begin{array}{l}29.764 \\
10.397 \\
11.420\end{array}$ & $\begin{array}{l}37 \\
49 \\
13\end{array}$ \\
\hline ota & & 2242.029 & 135.513 & 1110.355 & 51.581 & 100 \\
\hline a11. & & 26.691 & 1.613 & 13.218 & .614 & 194 \\
\hline
\end{tabular}

DIGESTIBLE HUTRIENTS CONSOMED*

PER 1,000 POUNDS IIVE WEIGHT

Total

1403.636

84.839

695.145

32.29362 .782

Ar. Dails

16.710

1.010

8.276

$.384 \quad .747$

\section{LOT II}

DIGESTIBIE NUTRIENTS CONSUNED*

$\begin{array}{lrrrrrr}\text { IG Grin } & 1234.0 & 1094.052 & 114.416 & 620.048 & 30.480 & 38.686 \\ \text { Ray } & 1373.5 & 1288.0 & 16.221 & 503.154 & 14.806 & 70.090 \\ \text { Total } & & 2382.052 & 130.637 & 1123.202 & 45.286 & 108.776 \\ \text { Ar. Daily } & & 28.358 & 1.555 & 13.371 & .539 & 1.295\end{array}$

DIGESTIBIS NUMRIENTS CONSUMAR*

PER 1,000 POUNDS IIVE WBIGHT

Total

Ar. Dailg
1426.105

17.096

1

Corn, oats, bran, 2-2-1

E Timothy hay

*pounds $\begin{array}{llll}78.211 & 672.447 & 27.11265 .123\end{array}$

$\begin{array}{llll}.931 & 8.005 & .323 \quad .775\end{array}$ 


$$
\text { GROUP B - c }
$$

PHOTOGRAPHED AT THE CLOSE OF THE EXPERIMENT, MARCH 15, 1915 LOT II FORGETME, NUMBER 2 IOT I JOSEPHINE, NUMBER I

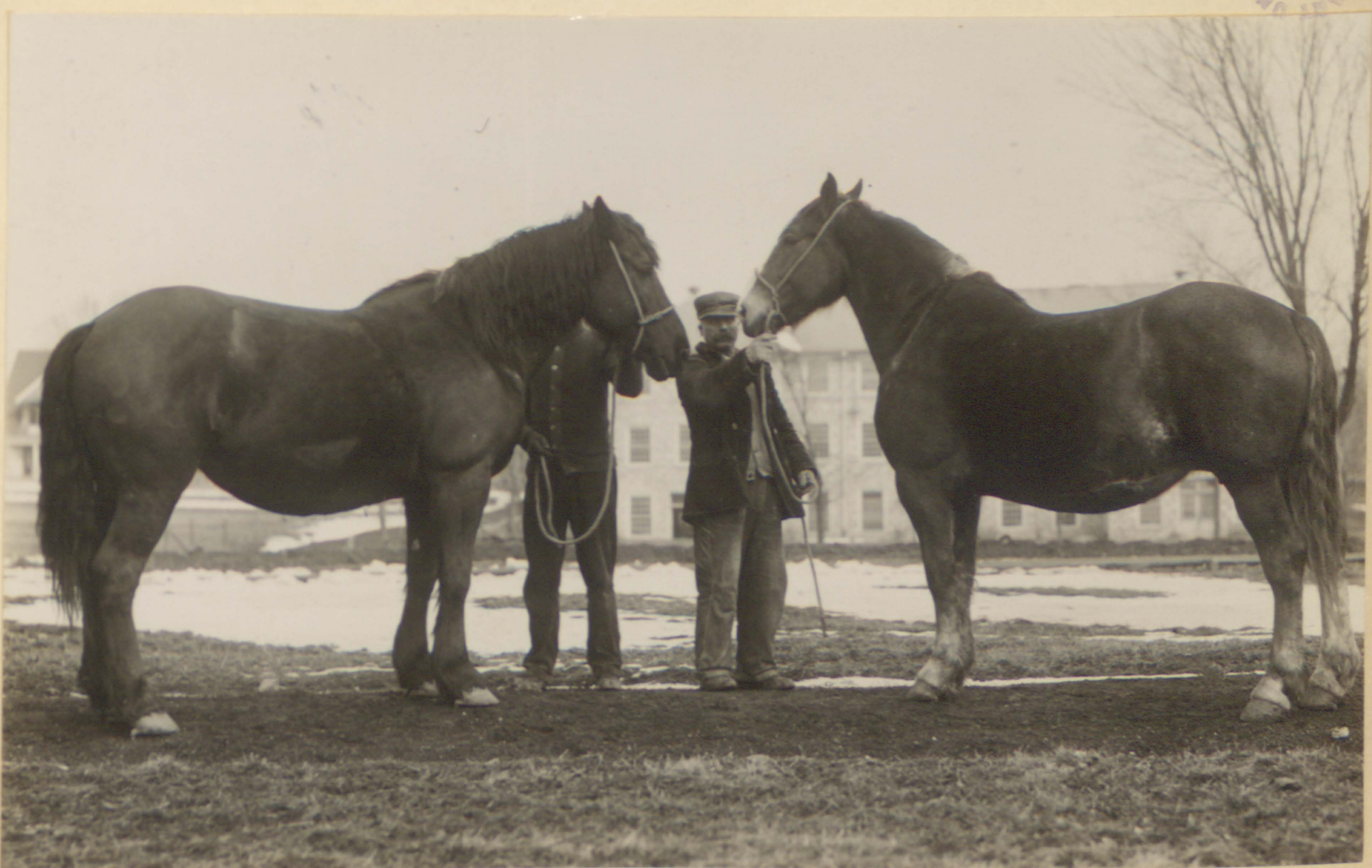

MATURE PREGNANT MARES PERFORMING AVERAGE WINTER FARM WORK NO SILAGE 
Tables VII and VII present the date for Group B-c-I pregnant mares, Josephire No. 1 and Forgette No. 2, performing average winter farm work.

Lot I was fod silage as a part ration. Lot II recelved no silage. Both lots were fed grain and timothy hay. These mares produced foals in the spring of 1914 and suckled them until the first of October. Josephine was bred Mag 2nd, 1914 and Forgette was bred lag 11, 1914.

Noither mare was fat at the beginning of the test. Mare No. 2 was in slightlg better condition of flesh than Mare No. 1. They were in medium breeding condition. Iot II wes somewhat heavier in weight than Lot $I$, at the beginning of the test. Their infitial weights were, Iot I 1572 pounds and Lot II 1637 pounds.

These mares were at work slightly more than one-half the time and gained in weight during the test. Iot I gained 81 pounds and Lot II gained 74 pounds during the 12 week period. Lot II consumed an average of .35 pounds of grain and 4.86 pounds of hay in excess of that consumed by Lot I. In addition to the grain and hay received, Lot I consumed an average of 8.27 pounds of $8118 g e$ daily.

Table VIf shows that Lot I received an arerage of .058 pounds of protein and .075 pounds of ash daily in excess of that consumed by Lot II. Iot II consumed an arerage of 2.667 pounds of dry matter, .153 pounds of carbohydrates 
and .101 pounds of fat more per day than Lot I.

When reduced to a thousand pound basis, Iot I consumed a dally average of .386 pounds of dry matter and .028 pounds of ash less than Iot II. An average of .079 pounds of protein, .271 pounds of carbohydrates and .06I pounds of fat was receired daily by Lot I in excess of that receired by Lot II.

At the close of the test both lots were in good breoding condition. Iot II was still in better condition of elesh. The condition of the hair and skin of the silage fed mare showed her to be in better thrift. 


\section{TABIS VIII}

GROUP B

MATURE HORSES AND YULES DOING AVERAGE TINTER FARM TORK

0. - 2 Pregnant mares

Lot I. Felibree, No. 3, Registered Percheron mare

Lot II. Finette, No. 4, Registered Percher on mare

TBED CONSULED*, WEIGHTS*AND WOBK HOURS BY WEEKLY PERIODS

$$
\text { IOT I LOT II }
$$

DATS $1_{\text {GRAIN }} 2_{\text {HAY }}$ SIIAGE WEIGHT ${ }^{3}$ TORK ${ }^{1}$ GRAIN $2_{\text {HAY WEIGHT }} 3_{\text {TORK }}$

1914

Dec.24

" 31

1915

Jan.

" 1494

" 21100

" 28100

Fob. 4100

" 11100

n 18100

" 25100

Mch. 4100

" 11100

ก 18100

Total 1158 BOURS

$\triangle B$

$\begin{array}{lllllll}37.5 & 1520 & 20 & 6.5 & 107 & 143.25 & 1420\end{array}$

$5_{1429}$

$\triangle B$

206.5

$78.75 \quad 1520$

$\begin{array}{lll}69.5 & 92 . & 1535 \\ 70 . & 89.25 & 1540\end{array}$

30

5.5

$107 \quad 144.25 \quad 1445$

$103 \quad 128.75 \quad 1452$

68.

70.

$97.5 \quad 1510$

$97.5 \quad 1550$

$97.75 \quad 1545$

91. $\quad 1560$

$97.5 \quad 1570$

$94.75 \quad 1575$

95.251610

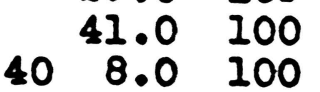

119. 1450

119.1425

$23.0 \quad 100 \quad 114.5 \quad 1480$

25.0100119 .1480

10.0100119 .1450

12.0100119 .1440

20. 100119.1450

1460

88.7515873015 .100 119. 1450

$30 \quad 5.5$

$\begin{array}{llll}5_{84} & 120 \quad 205 \quad 1217 & 1482.75\end{array}$

$5_{21} 120 \quad 205$

Daily

Are.

$13.71 \quad 9.96 \quad 12.59$

$14.49 \quad 17.65$

* Pound $s$

${ }^{1}$ corn, oats, bran, $2-2-1$

2 Timothy hay

3

A - hearg work, B - medium and light work

4

Intial woight

${ }^{B}$ Gain in wolght 


\section{TABIE VIIIA \\ LOT I}

DIGESTIBLE NUMRIENTS CONSUMED*

TOTAL FEED

CONSUNED

${ }_{\text {Hay }}^{1}$ Grain

S1lage

Total

Av. Da1ly

\section{0 \\ 863.5 \\ 1057.5}

DRY CARBO-

DRY MATIER PROTEIN HYDRATES PAT ASE

$\begin{array}{lllll}1021.351 & 106.813 & 578.845 & 28.454 & 36.115\end{array}$ $\begin{array}{lllll}809.747 & 10.198 & 316.326 & 9.309 & 44.064\end{array}$ $\begin{array}{lllll}409.358 & 18.845 & 230.419 & 17.364 & 20.114\end{array}$

$2240.456 \quad 135.856 \quad 1125.590 \quad 55.127 \quad 100.293$

$\begin{array}{lllll}26.672 & 1.617 & 13.402 & .656 & 1.194\end{array}$
DIGESTIBLE NUTRIENTS CONSUAED*

PER 1,000 POUNDS IIVE WEIGHT
Total

Av. Daily
1452.361

17.289
88.068

1.048
$724.741 \quad 35.73165 .014$

$8.625 \quad .425 \quad .774$

larain $_{\text {Hay }}$
Total
Av. Daily

$$
\begin{aligned}
& \text { ION II } \\
& \text { DIGESTIBLR NUTRIENTS CONSURED * } \\
& \begin{array}{l}
1217.0 \\
1482.5
\end{array} \\
& 1078.980 \\
& 112.840 \\
& \begin{array}{l}
611.506 \\
343.084
\end{array} \\
& 30.06038 .153 \\
& \begin{array}{llll}
17.508 & 343.084 & 15.981 \quad 75.652
\end{array} \\
& 2469.194 \\
& 130.348 \quad 954.590 \\
& 46.041113 .805 \\
& 29.395 \\
& 1.552
\end{aligned}
$$

DIGESTIBLB NUTRIENTS CONSUMOSD*

PER 1,000 POUNDS IIVE WEIGHT

Total

Av. Daily

$$
1709.177
$$

20.347

${ }^{1}$ corn, 08ts, bran, $2-2-1$

2 rimothy hag

*Pounds
$90.226 \quad 660.762 \quad 31.86978 .775$

$\begin{array}{llll}1.075 \quad 7.866 & .379 \quad .938\end{array}$


GROUP B - c

PHOTOGRAPHED AT THE CLOSE OF THE EXPERIMENT, MARCH 15, 1915 IOT II FINETTE, NUNBER 4 IOT I FELI BRES, NUMBER 3

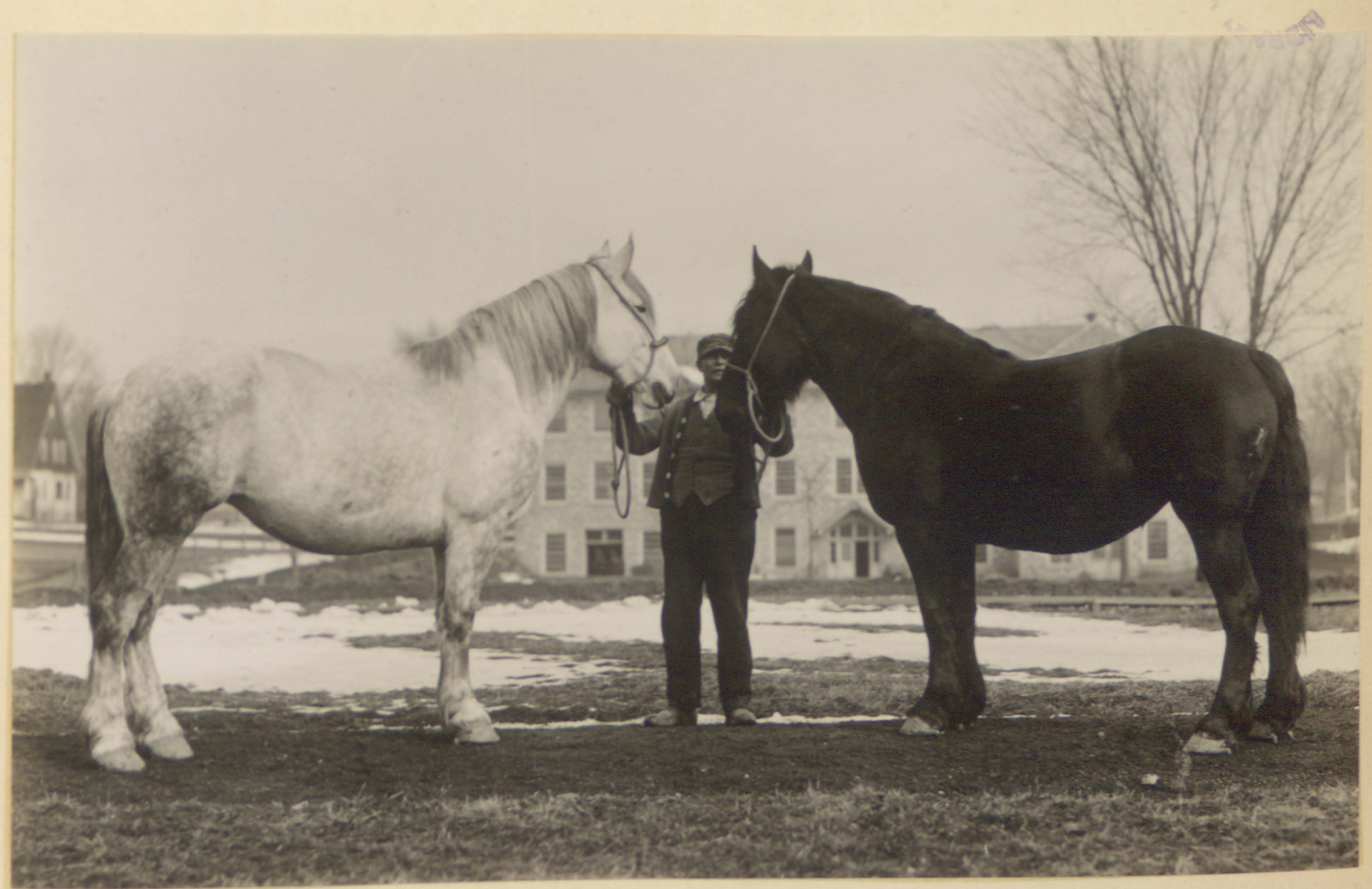

MATURE MARES PERFORMING AVERAGE WINTER FARM WORK MARE ON THE RIGHT PREGNANT. MARE ON LEFT NOT PREGNANT NO SILAGE SILAGE 
Tables VIII and VIII ${ }^{A}$ present the data for Group B-o-2, pregnant mare Felibree No. 3 and mare Finette No. 4, thought to have boen in foal at the beginning of the test. She proved later not to be in foal. This team performed average winter farm work.

Lot I received silage as a part ration. Lot II received no silage. Both lots were fed grain and timothy hay. Pinette, No. 4, produced a foal in the spring of 1914, and suokled her foal until october the first. Felibree, No. 3 was not pregnant the previous year. Felibree was bred May 29, 1914, and Finette was bred March 4, 1914.

At the beginning of the trial Pelibree was in slightIy the better condition, 88 is shown by the initial weights. The initiel weights were, Felibree, No. 31503 pounds and Pinette, No. 41429 pounds.

This team performed a total of 425 hours of work during the test.

A reviow of rable VIII shows that Iot II received dally an average of .78 pounds of grain and 7.69 pounds of haj in exces8 of Lot I. Iot I received an average of 12.59 pounds of $8 i 18 g 0$ daily.

$\Delta$ discussion of Table VIII $^{\Lambda}$ is omitted because these lots are not comparable. Lot I was in foal and Iot II proved not to be in roal.

There was a striking difference in the condition of these lots at the close of the test. Lot I was in decidedy 
the better condition. Her hair and skin indicated excellent thrift. 


\section{TABIS IX \\ GROUP C}

GRONING HORSES AND LULES

b. - I Draft foals

Lot I. Pure bred Percheron stud foll, No. 19, Dem, Pinette, Sire, Gardiner

Iot II. Pure bred Percheron filly foal, 10. 20, Dam Josephine, Sire, Gardiner.

FEED CONSURED*AND WBIGHTS*BY WERKLY PERIODS

\section{IOT I}

DATS ${ }^{2}$ GRAIN ${ }^{2}$ HAY 1914

De0.24

- 31

1915

Jan.

" 14

(i) 214

" 2814

Fob. 420

- 1128

" 1828

n 2528

Moh. 433

" 1135

ก 1835

30

21

$$
21
$$

$$
21
$$

25

35

35

35

35

35
SIILAG

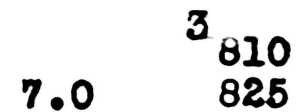

$35 \quad 31.75$

3536.

42.

42.

58.

70.

70.

Total $283 \quad 363 \quad 419.5$

Da115 Are.

\section{$3.37 \quad 4.32 \quad 5.0$}

*Pound 8

1

corn, oats, bran, 2-2-1

2

Llfalfa hay

3

Intigl weight

Gain in woight
IOT II

$1_{\text {GRAIN }} 2_{\text {HAY WEIGHT }}$

${ }^{3} 753$

$\begin{array}{lll}28 & 62.5 \quad 770\end{array}$

$\begin{array}{llll}800 & 28 & 65 . & 765\end{array}$

800

800

810

820

840

835

840

840

880

877

$28 \quad 72.5 \quad 795$

$2877 . \quad 805$

$28 \quad 77.815$

2877 . 825

$2877 . \quad 845$

2877.850

2877 . 840

3377.840

$35 \quad 75.25 \quad 825$

3577.885

${ }_{67} \quad 355 \quad 891.25{ }^{4} 132$

4.2210 .61 
TABIE I $x^{A}$

IOT I

DIGRTIBIR NUTRIENTS COHSURER*

TOTAL FEED

CONSUIED
283.0

363.0

419.5
DRY IATMER PROTEIN

250.905

340.984

162.388

754.277

8.979
CARBO-

HYDRATES RAT ASH

$\begin{array}{lllll}26.240 & 142.199 & 6.990 & 8.872\end{array}$

$38.028 \quad 144.786 \quad 1.194 \quad 31.635$

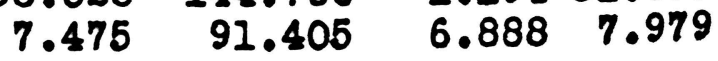

$71.743 \quad 378.390 \quad 15.072 \quad 48.486$

$.853 \quad 4.505 \quad .179 \quad .677$
DIGBSTIBLE NUTRIENTS CONSUMED*

PRR 1,000 POUNDS II VE WBIGHT

Total

17. Daily
86.43

1.029
455.892

5.427
18.15958 .658 $.216 \quad .698$

\section{IOT II}

DIGESTIBLE NUTRIEITS CONSULARD*

2 Gaj $_{\text {Hajn }}^{1}$

Total

Ar. Daily

\section{5. \\ 891.}

\begin{abstract}
314.739
836.961
\end{abstract}

$32.916 \quad 178.377$

$93.341 \quad 355.385$
8.76911 .129

2.93177 .651

$1151.700 \quad 126.257 \quad 533.762 \quad 11.70088 .780$

$$
13.711
$$

1.503

6.354 $.139 \quad 1.057$

DIGESTIBLE NUTRIENTS CONSURED*

PER 1,000 POUNDS IIVE WEIGHT

Total

Av. Da1ly
1421.642

16.927
156.823

1.855
658.754

7.842
14.440109 .570 $.172 \quad 1.304$

${ }^{2}$ corn, oats, bran, $2-2-1$

2 alfalfa hag

*pound 8 


$$
\text { GROUP } \mathrm{c}-\mathrm{b}-\mathrm{I}
$$

PHOTOGRAPHED AT THE CLOSE OF THE EXPER IMENT, MARCH 15, 1915 LOT II FILLY FOAL, NUMBER 20 - IOT I STUD FOAL, NUMBRR 19 DAM, JOSEPHINE, SIRR, GARDINER-DAM, PINETIE, SIRE, GARDINER

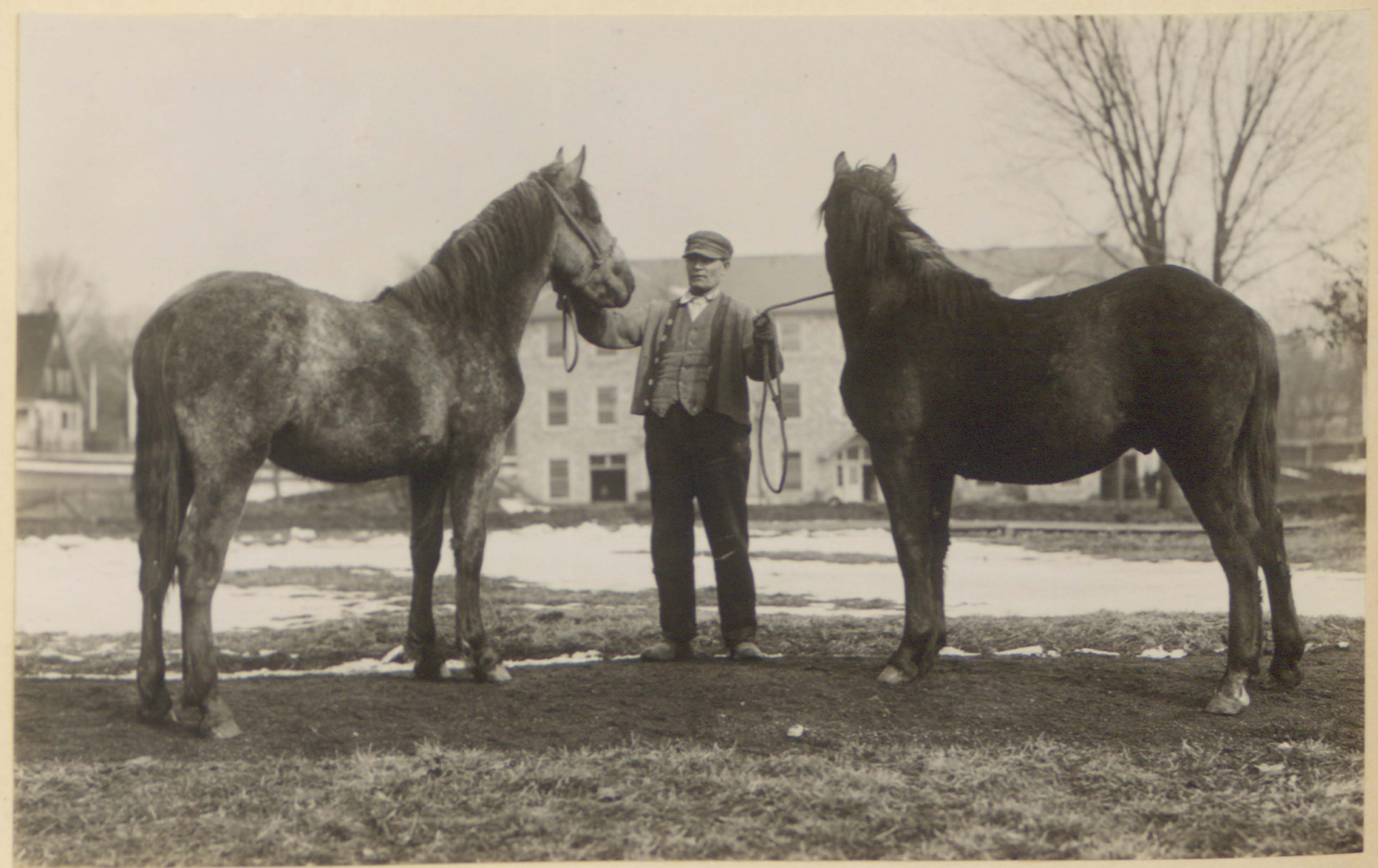

GROWING DRAFT FOALS 
Tables IX and IX ${ }^{A}$ present the data for Group $c-b-I$, growing draft forls. Lot I was fed silage as a part ration. Iot II receited no silage. Both lots were fed grain and alfalfa hag.

Lot I was foaled Pobruary 23, 1914 and Iot II was foaled March 12, 1914.

These draft fools had suckled their dams until October the first and had been on dry feed since that time. Iot I weighed 57 pounds more than Lot II at the beginning of the experiment.

Both loto were in thrifty growing condition at the beginning of the test. Iot I gained 57 pounds and Iot II gained 132 pounds during the fooding period.

Iot I oonsumed a daily average of 85 pounds of grain and 6.28 pounds of hay 1088 than Lot II. Lot I receired an arerage of 5 pounds of 811 age daily.

It will be noted from the table that Iot I consumed small quantities of the silage daring the first four weoks of the toet. No $801 n$ was made during this time. During the last two weeks of the trial, Lot I was fed 5 pounds of grain, 5 pounds of hay and 10 pounds of $8 i l a g \theta$ per day. Substantial gains were made during this time.

Table If shows that Lot II receired 4.732 pounds of dry matter, .65 pounds of protein, 1.849 pounds of carbohydrates, and .48 pounds of ash more per day than Lot I. Lot I receired - Os pounds of lat more daily than Iot II. 
When reduced to a thousand pound basis this table shovs that Lot II consumed an average daily excess of 6.108 pounds of dry matter, .826 pounds of protein, 2.415 pounds of carbohydrates and .606 pounds of ash over that consumed by Lot I. Lot I receired daily .044 pounds of fat more than Lot II. There was practically no difference in the condition of fleshing of these fools at the close of the test. They were in thrifty growing condition. The skin and hair of Lot I indoated that this foal was in slightly better thrift at the close of the test than Lot II. 
TABIE $x$

GROOP C

GROWING HORSES AND MULES

c. - I Ifght forls

Iot I Pure bred saddle filly foal Ruth Squirrel No. 17

Lot II Pure bred saddle filly foal Magna of Bst111 No. 18

IETE CONSUNED*AND WEIGHTS*BY WEEKCY PERIODS

\section{IOT I}

DAME ${ }^{1}$ GRAIN $2_{\text {HAY }}$ 1914 Deo. 24

ก 3128

1915

Jan. 72

- 2128

1 2828

Fob. 428

" 1128

- 18

" 25

Mch. 4

n 11
n

Total

Da11y

AVO.

35
IOT II

$2_{\text {HAY WBIGHT }}$<smiles>[13BH][18OH]</smiles>

35.75

$40 \quad 525$

22.5

38.25

40

40.5

41.75

41.5

42

42

42

48

468.25

5.57

4.26 .72

*Pounds

ICorn, 0ats, Bran, 2-2-1

8

Alfalfa hay

3

Initial weight

4

Gain in woight 
TABLE $x^{A}$

IOT I

DIGESTIBLE NUTRIENTS CONSURAD*

TOTAL FEED

CONSUIED

${ }_{\text {Hag }}^{1}$

S1lage

Total

Av. Daily

Total

Ar. Daily

350.75
394.0
468.25

468.25
DRY INATTER PROTEIN

310.971

370.108

181.260

862.339

10.266
CARBO

HYDRATES FAT ASH

$\begin{array}{llll}32.522 & 176.241 & 8.664 & 10.996\end{array}$

$41.275 \quad 157.151 \quad 1.296 \quad 34.337$

$\begin{array}{llll}8.324 & 102.027 & 7.689 & 8.906\end{array}$

$82.121 \quad 435.419 \quad 17.649 \quad 54.239$

$\begin{array}{llll}.978 & 5.184 & .210 \quad .646\end{array}$

DIGBSTIBLE NUTRIENMS CONSURED*

PER 1,000 POUNDS IIVE WEIGHT

$\begin{array}{lllll}1531.985 & 145.892 & 773.506 & 31.176 & 96.358\end{array}$

$\begin{array}{lllll}18.238 & 1.737 & 9.209 & .371 & 1.147\end{array}$

IOT II

DIGESTIBLE NUTRIENTS CONSUMER *

$\begin{array}{lrrrrrr}\frac{1}{\text { Grain }} & 353.0 & 312.966 & 32.730 & 177.372 & 8.719 & 11.067 \\ \mathcal{H}_{\text {Haj }} & 564.5 & 530.270 & 59.137 & 225.157 & 1.857 & 49.196 \\ \text { Total } & & 843.236 & 91.867 & 402.529 & 10.576 & 60.263 \\ \text { Av. Daily } & & 10.039 & 1.094 & 4.792 & .126 & .717\end{array}$

DIGRSTIBLE NUTRIENMS CONSURRD*

PER 1,000 POUNDS LIVE WEIGET

Total

Av. Daily
1537.573

18.304

1.994
$733.979 \quad 19.284 \quad 109.885$

8.739

${ }^{2}$ corn, 0ats, bran, 2-2-1

Ellfalfa haj

*Pounds 


$$
\text { GROUP } 0-c-1
$$

PHOTOGRAPHED AT THE CLOSE OF THE EXPERIMENT, MARCH 15, 1916

LOT II MAGNA OF RSTILL

NUMBER 18
IOT I RUTH SQUIRREL NUMBER 17

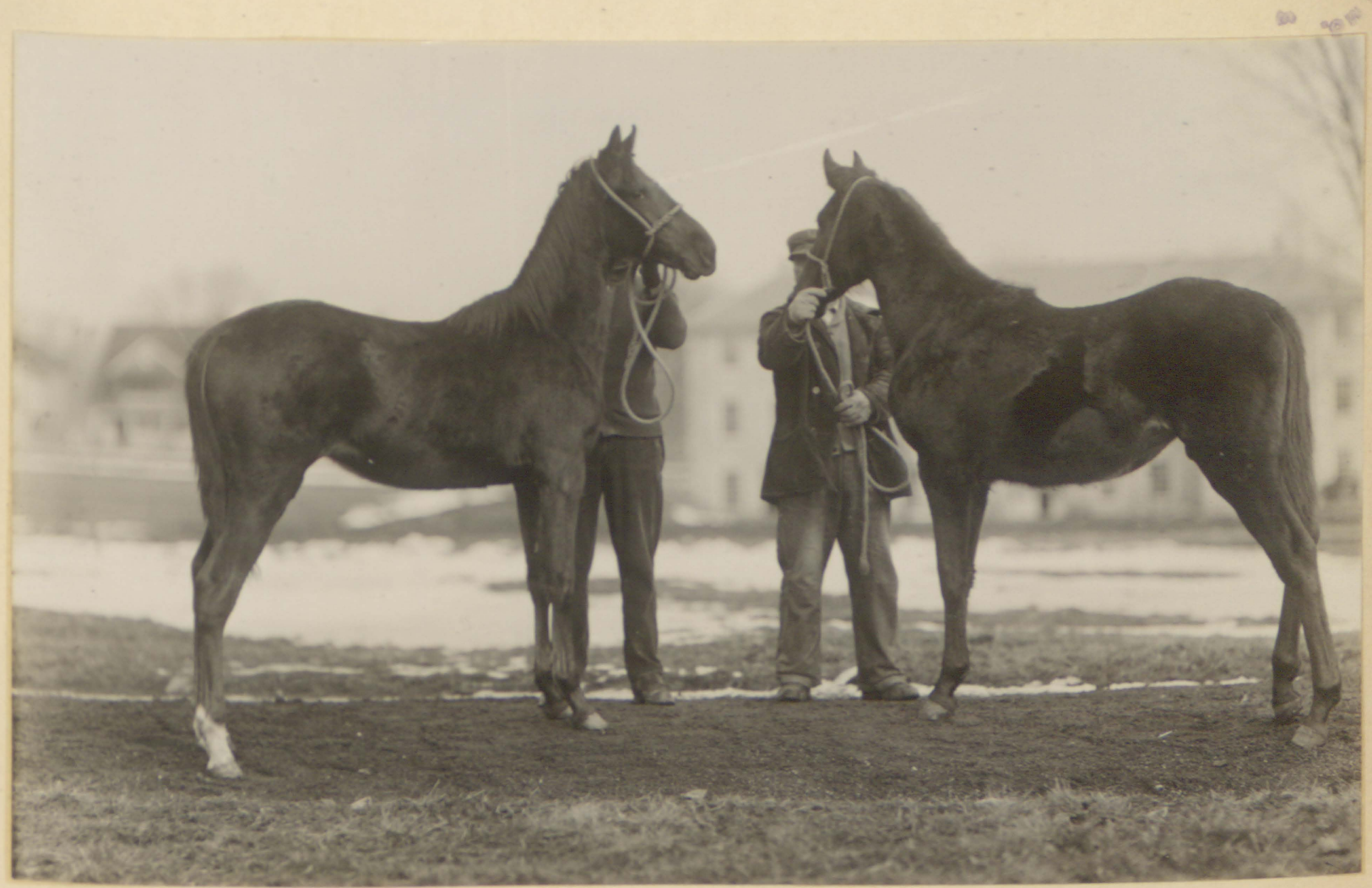

GROWING SADDLE POALS

NO SILAGE

SIIAGE 
Tables $X$ and $X^{A}$ present the data for Group $C-0-1$, growing sadde foals, Ruth Squirrel No. 17 and Nagna of Estill No. 18. Lot I was led silage as a part ration. Iot II received no slloge. Both lots were fed grain and alfalfa hag. Lot I was foalod April 13, 1914 and Lot II was forled Apr11 7, 1914.

Both lots suckled their dams until ootober 1st. They were on dry feed from that time until the beginning of the experiment. These foals were in medium growing condition at the beginning of the test. Iot I was a finer foal, being lighter in bone and finer in hair and skin than Iot II.

Lot I gained 48 pounds and Iot II gained 65 pounds during the feoding period.

Both lots consumed practicalig the same amount of grain da1ly. Lot I received 2.03 pounds of hay less per day than Lot II and was fed in addition an average of 5.57 pounds of sllage daily.

Prom Table $x^{A}$ it will be noted that Lot I reoeived an average of .227 pounds of dry matter, .392 pounds of carbohydrates and .084 pounds of fat more per dag than Lot II. Iot II reoeived daily an arerage of .116 pounds of protein and .071 pounds of ash more than Lot I.

The table shows when reduced to a thousand pound basis that Lot II received an average daily excess orer Iot I of .066 pounds of dry matter, .257 pounds of protein and .161 pounds of ash. The same table shows that lot I was fed per 
thousand pounds 11 ve weight .470 pounds of carbohydrates and .141 pounds of fat more per day than Lot II.

Lot II was in better thrift at the close of the trial

than Lot I. Her hair and skin were in better condition. This same condition was noted at the beginning of the trial. 


$$
\begin{aligned}
& \text { TABIE XI } \\
& \text { GROUP C } \\
& \text { GROTING HORSES AND MULES } \\
& \text { c. - } 2 \text { one and two year old light horses } \\
& \text { Lot I Sadde bred gelding Pepper No. } 15 \\
& \text { Lot II Standard bred filly Lady Adrance No.16 } \\
& \text { FEED COISUUCEN AND WEIGHTS* BY WEEKCY PERIODS }
\end{aligned}
$$

IOT I

$$
\begin{aligned}
& \begin{array}{l}
1914 \\
00.24
\end{array} \\
& \begin{array}{rrr} 
& 31 & 20 \\
& 1915 & \\
\text { Jan. } & 7 & 14 \\
\text { " } & 14 & 14 \\
\text { " } & 21 & 14 \\
& 28 & 14
\end{array} \\
& \text { Fob. } 4 \quad 14 \\
& \text { " } 1114 \\
& \text { n } 18 \quad 14 \\
& \text { " } 25 \quad 14 \\
& \text { Moh. } 414 \\
& \begin{array}{lll}
\text { " } 11 & 14
\end{array} \\
& \begin{array}{lllll}
n & 18 & 14 & 42 & 73.75
\end{array} \\
& \text { Total } \\
& 174 \\
& 45.0 \quad 28 \\
& 35 \quad 53 \\
& 36 \quad 70.5 \\
& 42 \quad 83.25 \\
& 42 \\
& 42 \\
& 42 \\
& 42 \\
& 42 \\
& 42 \\
& 42 \\
& 81.25 \\
& 79.25 \\
& 78.5 \\
& 82.5 \\
& 80.5 \\
& 73.25 \\
& 81 . \\
& \begin{array}{lll}
2.07 & 5.88 & 10.27
\end{array}
\end{aligned}
$$

WEIGHM

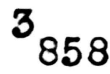

855

850

880

865

870

880

880

870

850

880

890

897

$4_{39} \quad 336$

28

28

28

28

28

28

28

28

28

28

28

IOT II 
TABIE XI

IOT I

DIGESTIBLE FUTRIENTS CONSUEDD

TOMAI TBED

CONSUNED

IGrain

Hay

silago

Total

Ar. Dails

Total

Ar. Da1ly
DRY WATTER PROTEIN

154.267

464.039

333.971

952.277

11.337
16.133

51.751

15.374

83.258

.991
CARBO-

HYDRATES FAT ASH

$87.430 \quad 4.298 \quad 5.455$

$197.037 \quad 5.325 \quad 43.053$

$187.985 \quad 14.166 \quad 16.410$

$472.452 \quad 23.78964 .918$

$5.624 \quad .283 \quad .773$
DIGESTIBLE HUTRIENMS CONSURED

PER 1,000 POUNDS IIVB WEIGHT

1093.114

95.571

1.138

13.013
27.30774 .519 $.325 \quad .887$

\section{IOI II}

DIGESMIBLE NUTRIENTS CONSURED

${ }_{\text {Eay }}^{1}$

Total

Av. Da1lg
336.0
744.0

297.894

698.876

996.770

11.866
$31.154 \quad 168.830$

79.941296 .752

8.29910 .534

8.02064 .840

16.31975 .374

1.299

5.543 $.194 \quad .897$

DIGESTIBLE NUTRIENTS CONSUNED

PRR 1,000 POUNDS IIVE TEIGH?

Total

Av. Daily
1287.467

15.327
140.912601 .364

1.678

7.159

21.09697 .356

$.251 \quad 1.159$

Icorn, oats, bran, $2-2-1$

2 Alfalfa hay 


$$
\text { GROUP C - c - } 2
$$

PHOTOGRAPHED AT THE CLOSE OF THE EXPERIMENT, MARCH 15, 1915 IOT II LADY ADVANCE, NUMBER 16 IOT I PEPPER, NUMBER 15

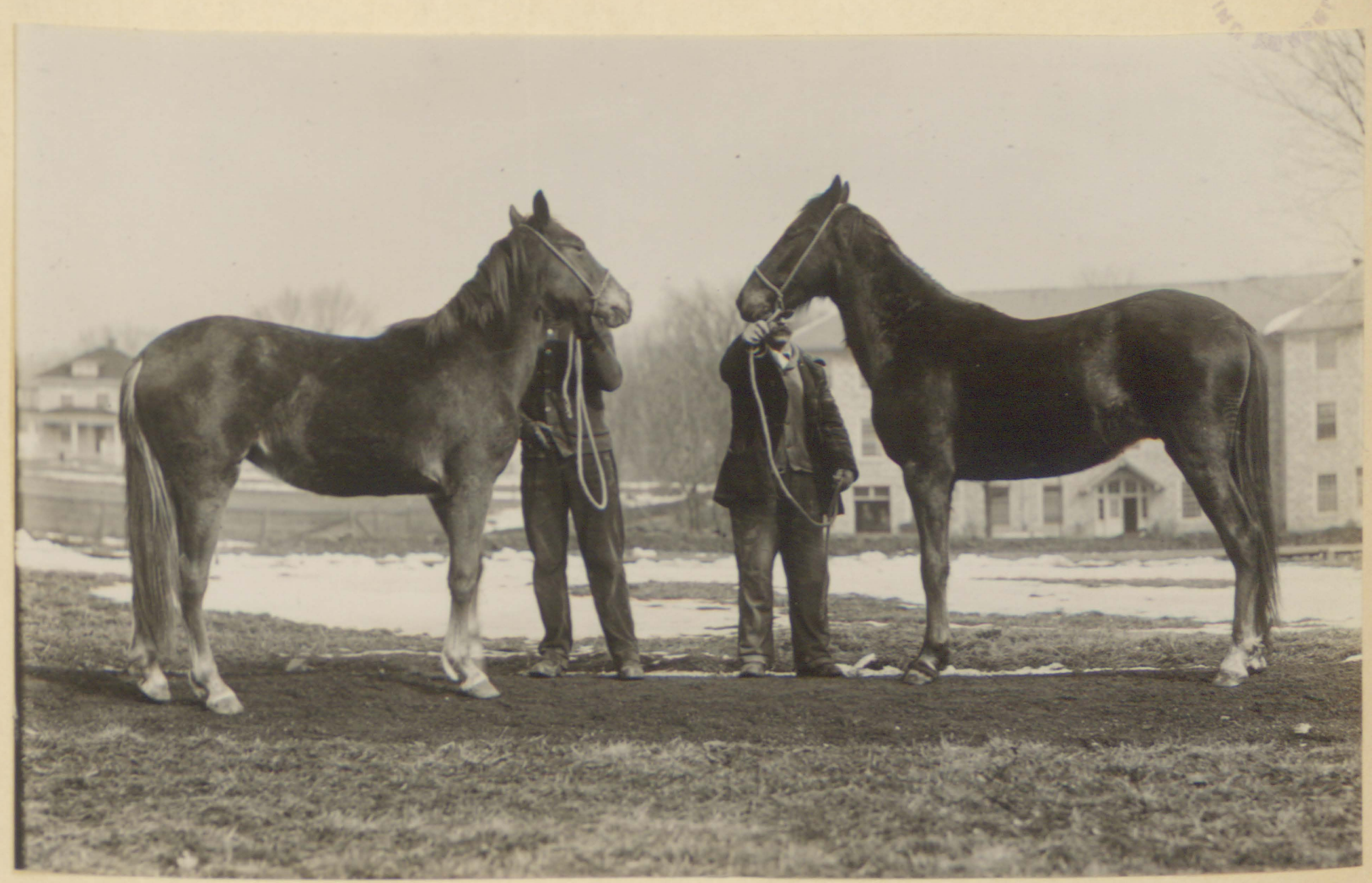

GROWING LIGHT HORSES

NO SIIAGE

SIIAAGE 
Tables XI and $X I^{A}$ present the data for Group $\mathrm{C}-0-2$, growing light horses, the two year old saddle bred gelding Popper, No. 15 and the yoarling stardard bred filly Iady Advanoe, No. 16 .

Lot I was fed 8 ilage 88 a part ration. Iot II recoived no gliage. Both lots were fed grain and alfalfa hay. Lot I was a year older than Iot II and was about 100 pounds heapier. These growing horses were on dry feed since they were brought in erom pesture october 30 th.

At the beginning of the test lot II was in much botter flesh than Lot I. She was more compact and more closely made throughout. Thej gained practically the same amount in the test. Lot I gained 39 pounds and Lot II gained 35 pounds. Lot II reoeived 1.93 pounds of grain and 2.98 pounds of hay more per day than Lot I. Lot I was led a daily average of 10.27 pounds of 8 ilage.

ravie XI shows that Lot II received a daily average of .629 pounds ary matter, .308 pounds of protein and .124 pounds of ash more than Lot I. Lot I consumed a daily excess of .081 pounds of carbohydrates and .089 pounds of fat orer that consumed by Lot II.

When reduced to a thousand pound basis Iot II recoived per day 2.314 pounds dry matter, .540 pounds protein, .703 pouhds carbohydrates and .272 pounds of ash more than Lot I. Lot I reooived da1Iy .074 pounds of fat more than Iot II. Lt the 01080 of the test Iot II was still in better 
condition of flesh than Lot I. The hair and skin of Lot I Indicated a little better thrift than Iot II.

\section{GROUP D}

IMRAATURE HORSES AND MULES DOING AVERAGE WINTER FARM WORK

b. Open mares

Lot I Josephe No. 5, Registered Percheron mare Lot II Mena No. 6, Registered Percheron mare

The data for this team of immature percheron mares Josephe, No. 5 and Nens, No. 6 performing average winter farm work is omitted.

Josephe, No. 5 refused the silage at the beginning of the test. After the amount of grain and hay fod was reduced this mare ato the 81lage. She was consuming about 8 pounds of ollage per day when she developed a case of destemper and indigestion and had to be taken off the experiment. 


\section{GROUP D - b}

PHOTOGRAPHED AT THE CLOSE OF THE EXPERIMEIT, MARCH 15, 1915 IOT II MENA, NUMBER 6 LOT I JOSEPHE, NUMBER 5

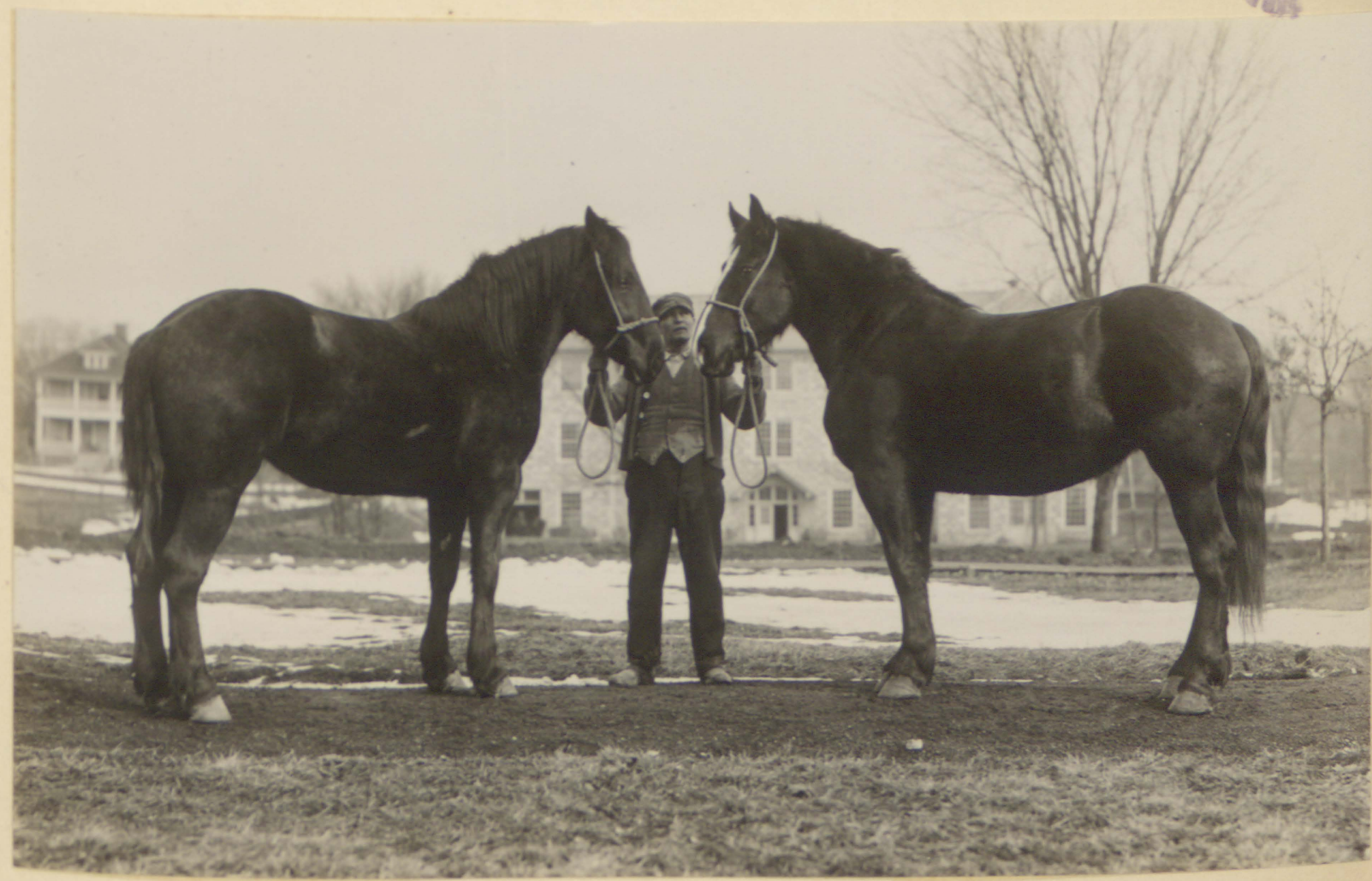

TMMATURE OPEN MARES* PERFORMING AVERAGE WINTER FARM WORK

\section{NO SILAGE}

\section{SIIAGB}

* These mares were taken off of the experiment January 20, 1915 for the reason that. Lot. I, Josephe No. 5 developed a case of distemper and indigestion. 


\section{TABIE XII}

IURMATURE IDIE HORSES AND MULES

\section{c. Pregnant mares}

Lot I Iona Rex No. 13, Registered sadale mare, two years old

Lot II Sateite No. 14, sadde bred mare, two jears old.

FEED CONSUIED*AND WEIGHTS ${ }^{*}$ BY WEEKLY PERIODS

\section{IOT I}

\section{DATE 1914}

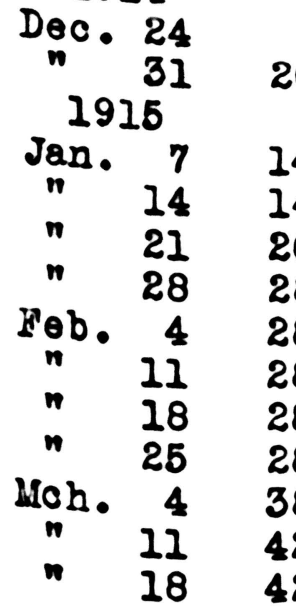

Total 330

\section{$2_{\text {HAY }}$}

50

35

35

35

35

35

35

35

35

35

35

35

435

\section{SIIAGE}

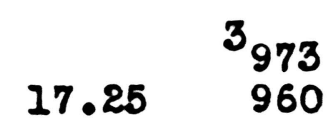

WEIG
$3_{973}$
960

$42.25 \quad 950$

64.

69.75

70 .

70 .

70 .

70.

67.5

80.

80.

78.5

IOT II

Da11g

AVo.

$$
3.93
$$

$5.18 \quad 9.28$

$4.0 \quad 14.42$

\section{*Pounds}

1

Corn, oats, Bran, 2-2-1

2

Alfalfa hay

3

Initial weight

4

Gain in woight 
TABLE XII

IOT I

DIGRSTIBLE NUTRIENTS CONSUMED*

DRY MATTER PROTEIN

\begin{abstract}
292.575
408.617

301.648
\end{abstract}

$30.598 \quad 165.815$

$13.886 \quad 169.791$

1002.840

11.937
$45.501 \quad 173.504$

CARBO-

HYDRATES FAT ASH

$\begin{array}{lllll}89.985 & 509.110 & 22.377 & 63.077\end{array}$

$\begin{array}{llll}1.071 & 6.061 & .266 & .751\end{array}$

$8.151 \quad 10.346$

$1.431 \quad 37.910$ 12.79514 .821
TOTAL FEED CONSUNED

IGrain $_{\text {Haj }}$
S1lage
Total
Ar. Da1ly

\begin{abstract}
330.0
435.0

779.25
\end{abstract}

DIGESTIBLE NUTRIENMS CONSUNED*

PER 1,000 POUNDS IIVE WEIGHT
Total

Av. Daily
1005.323

11.968
90.208

1.074
510.371

6.076

\section{IOT II}

DIGESTIBIE NUTRIENTS CONSUMED*
Iarain $_{\text {Haj }}$

Total

Av. Da11y

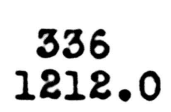

297.894
1138.492

1436.386

17.100 $\begin{array}{rr}31.154 & 168.830 \\ 126.969 & 483.418\end{array}$

$8.299 \quad 10.534$ $\begin{array}{rr}8.299 & 10.534 \\ 3.987 & 105.626\end{array}$ $158.123 \quad 652.248$ 1.882 7.765

12.286116 .160 $.267 \quad .768$

DIGESTIBLE NUTRIENTS CONSUMAD*

PER 1,000 POUNDS IIVE WEIGHT

Total

Av. Da1lJ
1329.661

15.829
146.375

1.743
603.788

7.188
11.373107 .530 $.135 \quad 1.280$

1

corn, oats, bran, 2-2-1

2

alfalfa haj

*Pounds 
GROUP E - b

PHOTOGRAPHED AT THE CLOSE OF THE EXPERIMENT, MARCH 15, 1915 LOT II SATELITE, NUMBER 14 IOT I IONA REX, NUMBER 13

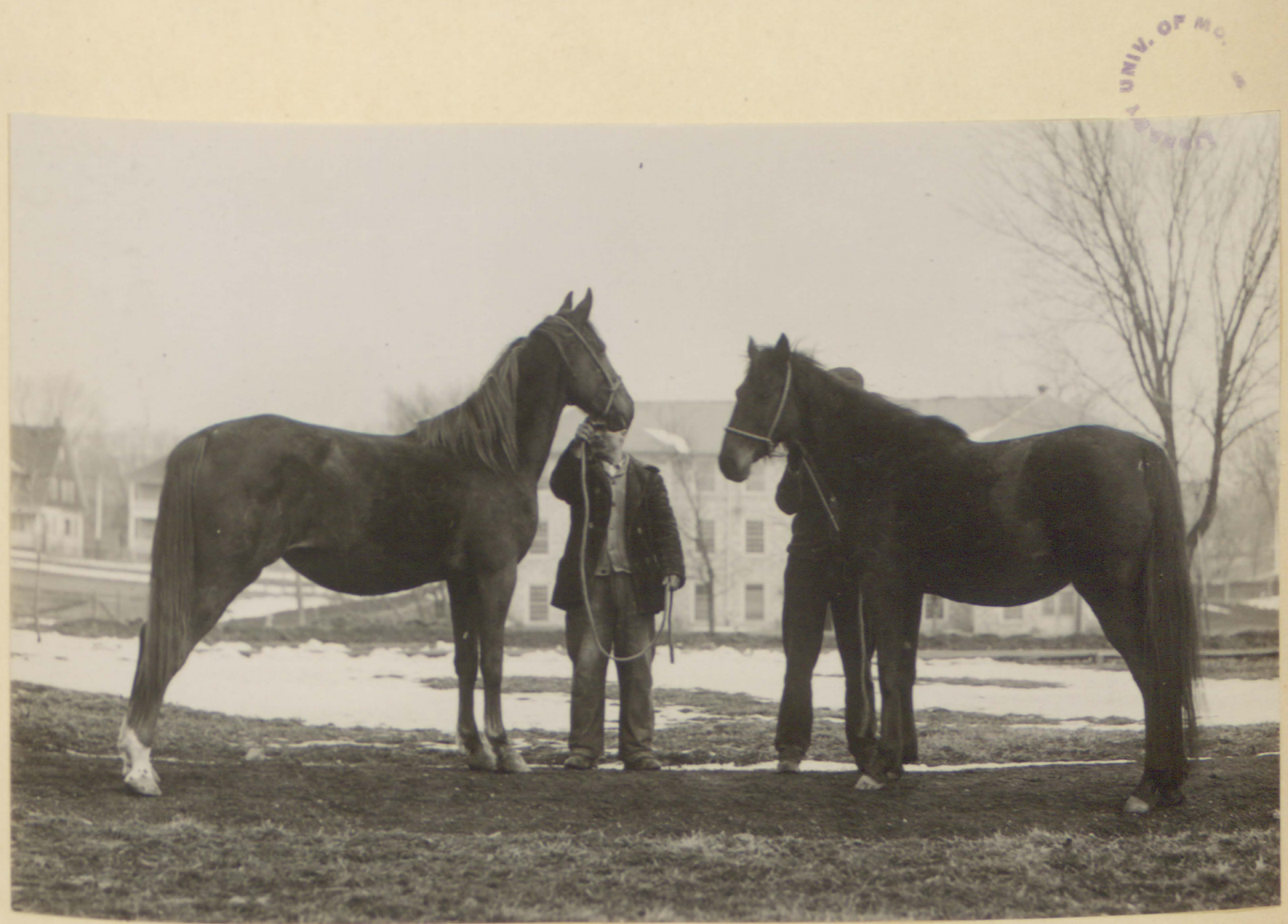

IMMATURE IDIE PREGNANT MARES

NO SILAGE

SIIAGE 
Tables XII and XII present the data for Group E-C, Immature idle pregnent mares Iona Rex, No. 13 and Satelite, No. 14.

Lot I was fed silage as a part ration. Lot II recoired no 81lage. Both lots were fed grain and alfalfa hay. These light mares were on pasture until ootober joth, after which time they were on dry feed. Iona Rex, No. 13, was bred May 1, 1914 and Satelite was bred iray 20, 1914. They had never been bred previous to this time.

Both mares were in medium breeding condition of flesh at the beginning of the test. Their initial weights were, Lot I, 973 pounds and Lot II, 1044 pounds. Lot I gaired 73 pounds and Lot II gained 96 pounds in the 12 week feeding period. Both lots consumed about the same amount of grain during the test. Lot I was fed 5.18 pounds of hay and 9.28 pounds of silage daily. Lot II received per dag 14.42 pounds of hay.

Lot II consumed daily an arerage of 5.163 pounds of dry matter, .811 pounds of protein, 1.704 pounds of carbohydrates and .632 pounds of ash more than Lot I. Lot I received .120 pounds of fat per day in excess of Lot II.

When reduced to a thousand pound basis, Lot II recoired 3.861 pounds of dry matter, .669 pounds of protein, 1.112 pounds of carbohydrates and .512 pounds of ash more than Iot I.

Lot I recoired per thousand pounds live weight . .132 
pounds of fat more per dey than Lot II. Both of these mares were in good breoding condition at the close of the test. The $81198 \theta$ fed mare was in better thrift than the other mare as was indicated by her hair and skin. 


\section{TABIE XIII}

SUMaSARY OF TOTAL FEED CONSURED*DURING 1914-15 TEST

NAVES

Ruth MeDonald No. 11

Mack No. 7

Jule No. 9

Josephine No. 1

Felibreo No. 3

Draft Poal 10.19

Ruth Squirrel No. 17

Pepper No. 15

Iona Rex 170.13
LOT I

$I_{\text {GRAIN }}$

378
1102.5
522.5
1205.
1152.
283.
350.75
174.
330.

Marthe 2nd No. 12

Cap No. 8

Kato No. 10

Porgette NO. 2

Pinette NO. 4

Draft Foal No. 20

Magna of Estili No. 18

Lady Advance No. 16

Satelite No. 14
IOI II

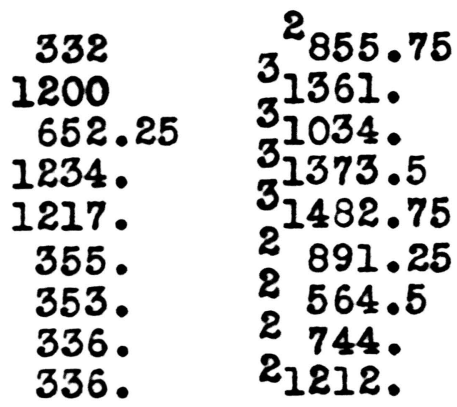

3780

3874.25

3682 .

3964.5

336.5

2363 .

394 .

2494 .

2435 .

SILAGE

919.25

930.25

676.

695.5

1057.5

419.5

468.25

862.75

779.25

${ }^{1}$ corn, 0ats, bran, $2-2-1$

2

Alfalfa hay

3

TImothy hay

*Pounds 
TABLE XIV

SUMOCARY OF ATERAGE FEED CONSUMRD* PRR DAY DURING 1914-1915 TEST IOT I

\section{NALE}

Ruth MoDonald No. 11

Maok No. 7

Jale No. 9

Josephine No. 1

Folibreo No. 3

Draft Foal No. 19

Ruth Squirrel No. 17

Popper No. 15

Iona Rex No. 13 $1_{\text {GRAIN }}$

\begin{tabular}{|c|}
\hline $\begin{array}{r}4.5 \\
13.16 \\
6.21 \\
14.34 \\
13.71 \\
3.37 \\
4.16 \\
2.07\end{array}$ \\
\hline
\end{tabular}

SIIAGE

10.94

11.07

8.05

8.27

12.58

5.0

5.57

10.26

IOI II

Marthe 2nd No. 12

Cap No. 8

Kato No. 10

Porgette NO. 2

Pinette No. 4

Draft Foal No. 20

Magna of Eatili No. 18

Iady Adranoe No. 16

Satelite No. 14

$\begin{array}{rl}3.95 & 210.19 \\ 14.28 & 316.2 \\ 7.76 & 312.31 \\ 14.69 & 316.34 \\ 14.49 & 317.64 \\ 4.22 & 210.6 \\ 4.2 & 2 \\ 4.0 & 26.71 \\ 4.0 & 21.85 \\ 4.0 & 24.42\end{array}$

${ }^{1}$ corn, oats, bran, $2-2-1$

${ }^{2}$ Alfalfa

3 Iimothy

*Pounds 
Tables XIII and XIV present a summary of the total grain, hay and silage, and the average grain, hay and silage consumed per day by all the horses during the 1914-1915 test. The horses in lot I received silage as a part ration. The horses in Lot II received no silage.

The horses in Lot I received an average of 7.272 pounds of grain, 7.698 pounds of hay and 8.101 pounds of silage per day during the twelve weok feeding period. The horses in Lot II consumed an average of 7.954 pounds of grain and 12.584 pounds of hay during the test. The horses in Lot I made a total gain of 421 pounds and the horses in Lot II made a total gain of 409 pounds during the feoding period.

Comparing the average feed consumed per day by both lots it will be noted that an average of 8.101 pounds of silage led to horses in Lot I repleced .682 pounds of grain and 4.886 pounds of hag in the ration. 
TABLE XV

SUICAARY OF TOMAL DIGESTIBLR NUTRIENTS CONSUNED* DURING 1914-1915 TBST IOT I

NANBS DRY MATTER

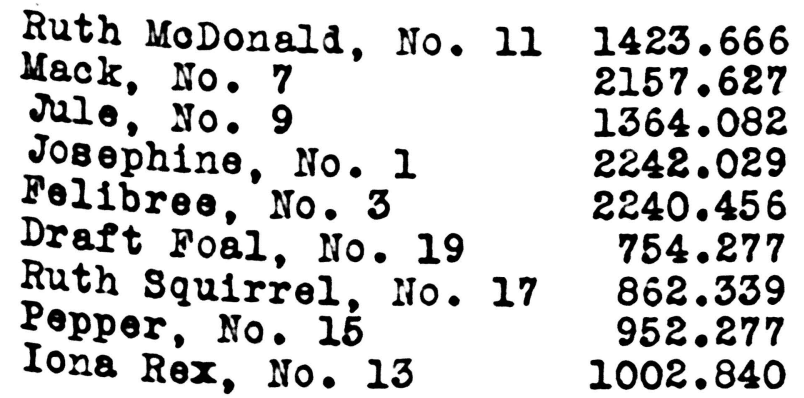
2157.627

1364.082

2242.029

2240.456

754.277

862.339

952.277

1002.840
$133.142 \quad 701.337$

129.1291077 .021

68.529659 .452

$135.513 \quad 1110.355$

135.8561125 .590

$71.743 \quad 378.390$

$82.121 \quad 435.419$

$83.258 \quad 472.452$

89.985

509.110

FAT ASH

$27.997 \quad 97.311$

$51.934 \quad 96.892$

$31.342 \quad 64.021$

51.581100 .283

55.127100 .293

$15.072 \quad 48.486$

$17.649 \quad 54.239$

23.78964 .918

$23.377 \quad 63.077$

\author{
Nartha 2nd, No. 12 \\ Cap, No. 8 \\ Kate, NO. 10 \\ Porgette, No. 2 \\ Pinette, No. 4 \\ Draft Hoal, No. 20 \\ Magna of Estili, 170.18 \\ Iady Adranco, No. 16 \\ Satolite, No: 14
}

$\begin{array}{rrr}1098.197 & 120.431 & 508.145 \\ 2340.186 & 127.337 & 1101.539 \\ 1559.912 & 72.589 & 706.521 \\ 2382.052 & 130.637 & 1123.202 \\ 2469.194 & 130.348 & 954.590 \\ 1151.700 & 126.257 & 533.762 \\ 843.236 & 91.867 & 402.529 \\ 996.770 & 109.095 & 465.582 \\ 1436.386 & 158.123 & 652.248\end{array}$

$11.015 \quad 84.987$

$44.312 \quad 107.072$

$27.257 \quad 73.213$

45.286108 .776

46.041113 .805

$11.700 \quad 88.780$

$10.576 \quad 60.263$

$9.431 \quad 75.374$

$12.286 \cdot 116.160$

*Pounds 
TABLE XVI

SUMCLARY OF AVERAGE DAILY DIGESTIBLE NUTRIENSS CONSURRD* DURING 1914-1915 TEST

IOT I

INAVBS

DRY MATMER PROTEIN

CARBO BYDRATES

FAT ASH

Ruth MoDonald, No. Il Mack, No. 7 Jule, No. 9 Josephine, No. 1 Felibree, No. 3 Draft Foal, No. 19 Ruth squirrel, No. 17 Pepper, 110. 15

Iona Rex, No. 13

16.948
25.686
16.239
26.691
26.672
8.979
10.266
11.337
11.937

1.585
1.537
.816
1.613
1.617
.853
.978
.991
1.071

8.349

12.822

7.851

13.218

13.402

4.505

5.184

5.624

6.061
$.333 \quad 1.158$

$.618 \quad 1.153$

$.373 \quad .762$

$.614 \quad 1.194$

$.656 \quad 1.194$

.179

.210

.283

.266

\section{IOT II}

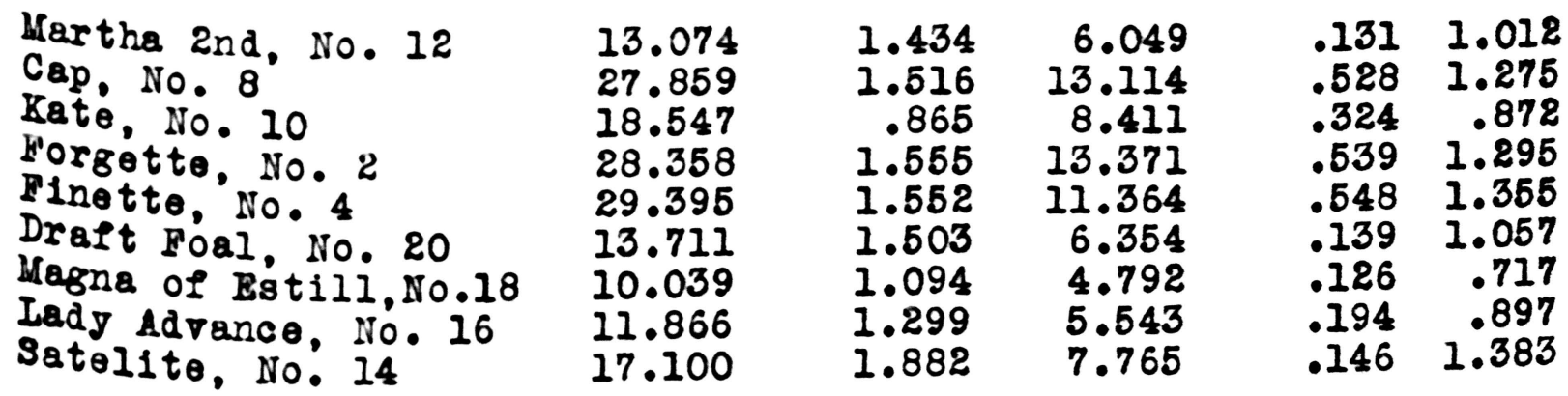


Tabies XV and XVI present a summary of the total digestible nutrients and the average daily digestible nutrients consumed by the horses in the 1914-1915 test.

Lot I received silage as a part ration. Lot II recoived no silage.

The horses in this test received on an average, the following nutrients per day:

Dry Matter Protein Carbohydrates Fat Ash

$\begin{array}{llllll}\text { Lot I } & 17.195 & 1.229 & 8.557 & .392 & .912 \\ \text { Lot II } 19.105 & 1.411 & 8.529 & .297 & 1.096\end{array}$

This table shows that the horses in Lot I received an arerage of 1.910 pounds of dry matter, .182 pounds of protein and .184 pounds of ash per day less than the horses in Lot II. The horses in Lot I received slightly more energy in the form of .028 pounds of carbohydrates and .095 pounds of fat daily. 
TABLE XVII

SUMAARY OF DIGESTIBLE NUTRIENTS CONSURED* PER THOUSAID POUNDS IIVE WEIGEM DURING 1914-1915 TEST

\begin{tabular}{|c|c|c|c|c|c|}
\hline WAISE & DRY MATMER & PROTEIN & $\begin{array}{l}\text { CARBO- } \\
\text { HYDRATES }\end{array}$ & FAT & ASH \\
\hline $\begin{array}{l}\text { Donald, No. } 11 \\
\text { 10. } 7 \\
\text { 10. } 9 \\
\text { ne, No. } 1 \\
0, \text { No. } 3 \\
\text { od, No. } 19 \\
\text { ulrrel, No. } 17 \\
\text { No. } 15 \\
x, \text { No. } 13\end{array}$ & $\begin{array}{r}1145.301 \\
1601.053 \\
947.799 \\
1403.636 \\
1452.361 \\
908.767 \\
1531.985 \\
1093.114 \\
1005.323\end{array}$ & $\begin{array}{r}107.109 \\
95.819 \\
47.616 \\
84.839 \\
88.068 \\
86.437 \\
145.892 \\
95.571 \\
90.208\end{array}$ & $\begin{array}{l}564.207 \\
799.196 \\
465.152 \\
695.145 \\
724.471 \\
455.892 \\
773.506 \\
542.325 \\
510.371\end{array}$ & $\begin{array}{l}22.526 \\
38.537 \\
21.777 \\
32.293 \\
35.731 \\
18.159 \\
31.176 \\
27.307 \\
22.433\end{array}$ & $\begin{array}{l}78.284 \\
71.898 \\
44.481 \\
62.782 \\
65.014 \\
58.658 \\
96.358 \\
74.519 \\
64.527\end{array}$ \\
\hline
\end{tabular}

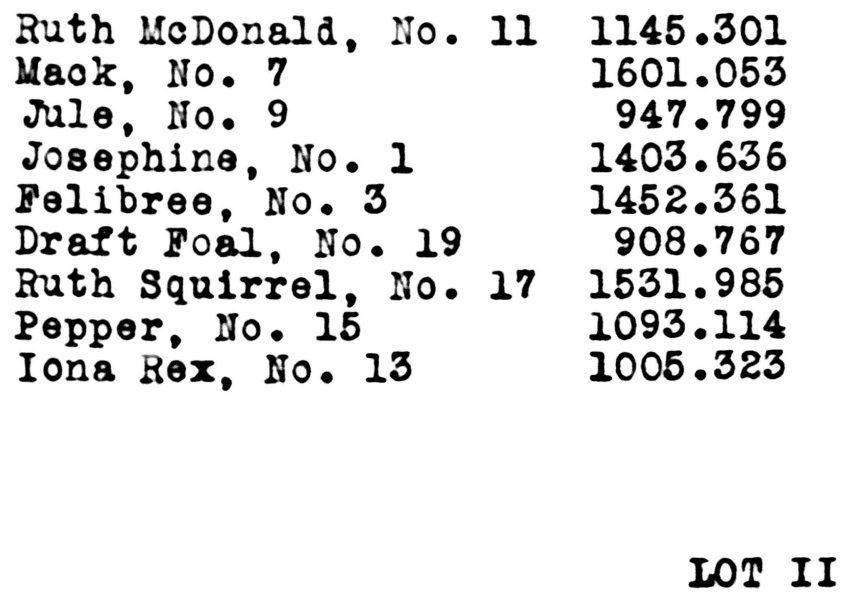

\begin{tabular}{|c|c|c|c|c|}
\hline 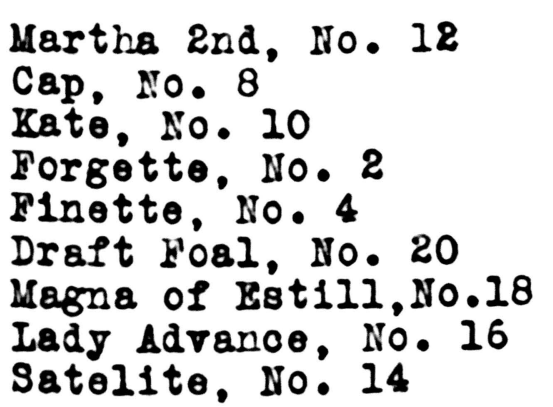 & $\begin{array}{r}899.579 \\
1777.122 \\
1308.486 \\
1426.105 \\
1709.177 \\
1421.642 \\
1537.573 \\
1287.467 \\
1329.661\end{array}$ & $\begin{array}{r}98.650 \\
96.699 \\
61.051 \\
78.211 \\
90.226 \\
155.823 \\
167.512 \\
140.912 \\
146.375\end{array}$ & $\begin{array}{l}416.243 \\
836.502 \\
510.252 \\
672.447 \\
660.762 \\
658.754 \\
733.979 \\
601.364 \\
603.788\end{array}$ & $\begin{array}{r}9.02369 .616 \\
33.65081 .310 \\
22.89361 .491 \\
27.11265 .123 \\
31.86978 .775 \\
14.440109 .570 \\
19.284109 .885 \\
12.18297 .356 \\
11.373107 .530\end{array}$ \\
\hline
\end{tabular}

*Pound 8 
TABIS XVIII

SURCARY OF AVERAGE DAIIY DIGESTIBIE NUMRIENTS CONSUINE* PRR THOUSAND POUNDS IIVB HRIGHI DURING 1914-1915 TEST

\section{LOT I}

NANS

DRY MATTER PROTEIN

CARBOFAT ASH HYDRATES

Ruth MeDonald, No. 11 Mack, No. 7

Jule, No. 9

Josephine, No. 1

Felibree, No. 3

Draft Foal, No. 19

Ruth Squirrel, No. 17

Pepper. No. 15

Iona Rex, No. 13
13.635

19.060

11.283

16.710

17.289

10.819

18.238

13.013

11.968

\subsection{5}

1.141 .567

1.010

1.048

1.029

1.737

1.138

1.074
6.717

9.514

5.538

8.276

8.625

5.427

9.209

6.456

6.076
.268

.459

.259

.384

.425

.216

.371

.325

.267
.932

.856

.530

.747

.774

.698

1.147

.887

.768

IOT II

\begin{tabular}{|c|c|c|c|c|}
\hline $\begin{array}{l}\text { Martha 2nd, No. } 12 \\
\text { Cap, No. } 8 \\
\text { Kate, No. } 10 \\
\text { Forgette, No. } 2 \\
\text { Pinette, No. } 4 \\
\text { Draft Foal, No. } 20 \\
\text { Hagna of Estili, No.18 } \\
\text { Lady Advance, No. } 16 \\
\text { Satelite, No. 14 }\end{array}$ & $\begin{array}{l}10.709 \\
21.156 \\
15.577 \\
17.096 \\
20.347 \\
16.927 \\
18.304 \\
15.327 \\
15.829\end{array}$ & $\begin{array}{r}1.174 \\
1.151 \\
.726 \\
.931 \\
1.075 \\
1.855 \\
1.994 \\
1.678 \\
1.743\end{array}$ & $\begin{array}{l}4.955 \\
9.958 \\
6.074 \\
8.005 \\
7.866 \\
7.842 \\
8.738 \\
7.159 \\
7.188\end{array}$ & $\begin{array}{l}.107 \\
.401 \\
.273 \\
.323 \\
.379 \\
.172 \\
.230 \\
.145 \\
.135\end{array}$ \\
\hline
\end{tabular}

*Pound 8 
Tables XVII and XVIII present a summary of the total and average dails digestible nutrients consumed per thousand pounds live weight by the horses in the 1914-1915 test. Iot I recelved sllage as a part ration. Lot II receired no silage. The horges in this test received on an average, the following nutrients dails per thousand pounds live weight:

Dry Matter Protein Carbohydrates Fat Ash

$\begin{array}{llllll}\text { Lot I } & 14.668 & 1.113 & 7.315 & .330 & .815 \\ \text { Lot II } & 16.808 & 1.370 & 7.532 & .241 & 1.044\end{array}$

This table shows that the horses in Lot I consumed an average per thousand pounds live weight of 2.14 pounds dry matter . 257 pounds of protein, .217 pounds carbohydrates and .229 pounds of ash per day less than the horses in Lot II. The horses in Lot I consumed an average of .089 pounds of fat per day more than the horses in Iot II.

The horses in Lot I gained a total of 421 pounds and the horses in Lot II gained a total of 409 pounds during the test. 
THR 1915-1916 TIST 


\section{INTRODUCTION}

The second experiment was conducted in order to secure more data and information on the subject of silage as a part ration for horses and mules of various ages under varying conditions than was possible to secure from a single trial. The 1915-1916 experiment was started December 17, 1915 and closed Larch 10, 1916. The same kinds of feeds were used in both tests. The general manggement of the horses was the same in both tests except that silage was fed at night and hay in the morning in the second trial. The horses were fed small quantities of silage before the test began. The quality of the sllage fed during the 1915-1916 experiment was not as good as that fed in the 1914-1915 experiment. 
TABIE IIX

CHEMICAL aNATYSIS OF FGEDS USED*

\begin{tabular}{|c|c|c|c|c|c|c|c|c|c|}
\hline & THEED & \% NITRO- & $\begin{array}{l}\text { \% CRUDE } \\
\text { PROIIEIN }\end{array}$ & $\%$ WATER & \& TAT & $\%$ ASH & $\begin{array}{l}\% \text { FIBER } \\
\text { CRUDE }\end{array}$ & $\%$ N.I.E. & $\begin{array}{l}\% \text { CARBO- } \\
\text { HYDRATES }\end{array}$ \\
\hline $\begin{array}{l}\text { Rough- } \\
\text { O\&@8 }\end{array}$ & $\begin{array}{l}\text { S1lage, a1r dry } \\
\text { S1lage, fresh } \\
\text { Timothy } \\
\text { Alfalfa }\end{array}$ & $\begin{array}{r}1.315 \\
.496 \\
.752 \\
2.330\end{array}$ & $\begin{array}{r}8.219 \\
3.100 \\
4.700 \\
14.563\end{array}$ & $\begin{array}{r}5.543 \\
64.359 \\
6.643 \\
6.833\end{array}$ & $\begin{array}{l}3.610 \\
1.362 \\
1.995 \\
2.878\end{array}$ & $\begin{array}{l}5.200 \\
1.962 \\
4.810 \\
9.128\end{array}$ & $\begin{array}{r}21.068 \\
7.950 \\
32.430 \\
29.110\end{array}$ & $\begin{array}{l}56.360 \\
21.267 \\
49.422 \\
37.488\end{array}$ & $\begin{array}{l}77.428 \\
29.217 \\
81.852 \\
66.598\end{array}$ \\
\hline $\begin{array}{l}\text { Concen- } \\
\text { trates }\end{array}$ & $\begin{array}{l}\text { Corn } \\
\text { Oats } \\
\text { Oats } \\
\text { Bran }\end{array}$ & $\begin{array}{l}1.582 \\
1.900 \\
1.837 \\
2.605\end{array}$ & $\begin{array}{r}9.888 \\
11.875 \\
11.481 \\
16.281\end{array}$ & $\begin{array}{l}9.263 \\
8.135 \\
8.168 \\
8.810\end{array}$ & $\begin{array}{l}4.235 \\
5.150 \\
5.063 \\
3.813\end{array}$ & $\begin{array}{l}1.298 \\
3.545 \\
3.055 \\
6.893\end{array}$ & $\begin{array}{r}2.200 \\
12.770 \\
12.820 \\
3.813\end{array}$ & $\begin{array}{l}73.116 \\
58.525 \\
59.413 \\
60.390\end{array}$ & $\begin{array}{l}75.316 \\
71.295 \\
72.233 \\
64.203\end{array}$ \\
\hline
\end{tabular}

*Department of Agricultural Chemistry, University of Missouri The Agricultural Chemistry Department furnished the analysis of the air dry material. The author made the calculations for the composition of the frosh silage, protein, nitrogen free extract and carbohydrates. 


\section{CHIMISTS' DATA}

A comparison of the chemical analysis of the feeds used in both trials show that the fresh silage fed during the second test was decidedly lower in percentage of crude protein, fat, nitragen free extract and carbohydrates than the silage fed in the first test. It was higher in percentage of moisture, ash and orude fiber.

The timothy hay was considerably lower in crude protein, fat, and ash than that fed during the previous trial. The percentaze of moisture, fiber, nitrogen free extract and carbohydrates was practically the same.

In ocmparison with the previous trial the alfalfa hay used was slightly higher in water content, crude protein, lat, ash, and nitrogen pree extract. It was slightly lower in crude piber and carbohydrates.

The oorn fed was slightly lower in orude protein and moisture and practically the same in ash as the corn fed during the previous trial. It was higher in fat, orudo fiber, nitrogen ereo extract and carbohydrates.

A comparison of the oats with those fed in 1914-1915 ahows that they were slightly lower in crude protein, moisture and ash. They were olightly higher in fat, crude fiber, nitrogen freo extract and carbohydrates.

The bran which was fed contained less crude protein, water, nitrogen free extract and oarbohydrates than the bran fed in the previous trial. It conteined deoidedly less orude protein and slightly more ash. 
TABIE $X X$

AVERAGE DIGESTIBIIITY OF FGEDS ${ }^{2}$ - EXPERIMENTS WITH HORSES

$$
\text { \% PROTEIN \% FIBER \% N.F.E.\%FAT AUTHORITY }
$$

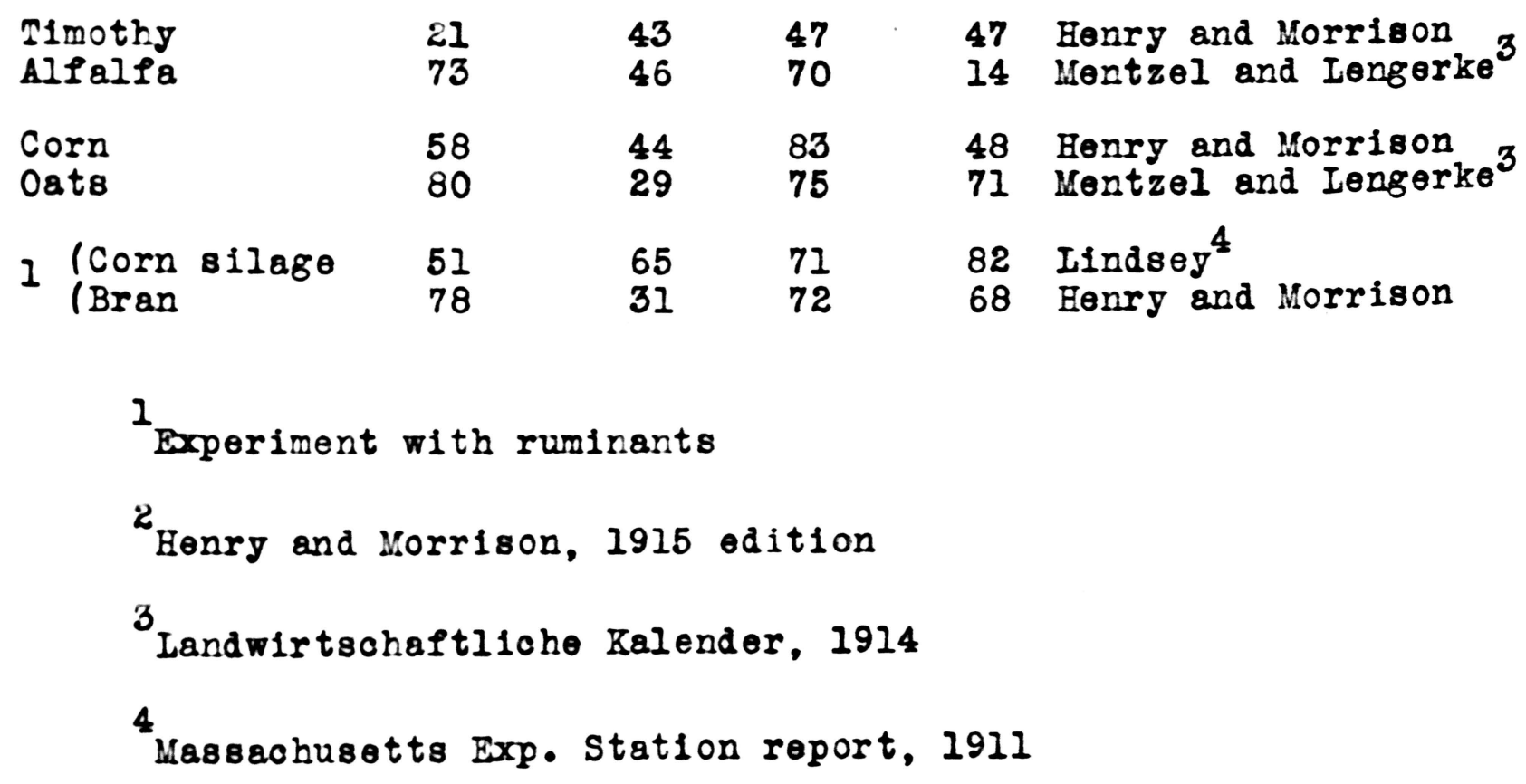

For discussion of Table JX refer to Table II. 
TABIE XXI

DIGESTIBLE TUMRIENTS IN 100 POURDS OF FERDS USED PEAD DRY PROTEIN FIBER N.F.E.
LATTER CARBO-
HYDRATES

FAT ASH

Corn silage,

Timothy

Presh

Alfalfa

Corn

Oets

Oats

Bran
35.641

93.357

93.167

1.581

$0.987 \quad 13.945$

10.63113 .391

90.737

91.865

91.832

91.190
5.735

9.500

9.185

12.699
.968

3.703

3.718

1.182
15.100

23.228

20.268

37.173

26.242

39.633

60.686

43.894

61.654

47.597

44.560

43.481
1.1171 .962

0.9384 .810

.4039 .128

2.0331 .898

3.6573 .545

3.5953 .055

2.5936 .893

NUTRIENTS IN 100 POUNDS OF GRAIN FED COMPOSED OF 2 POUNDS CORN, 2 POUNDS OATS, I POUND BRAN BY WEIGHT

Grain Fed December 17, 1915 - January 20, 1916

$\begin{array}{lllllll}91.279 & 8.634 & 2.105 & 50.528 & 52.633 & 2.795 & 3.316\end{array}$

Grain Fod January 21, 1916 - Maroh 10, 1916

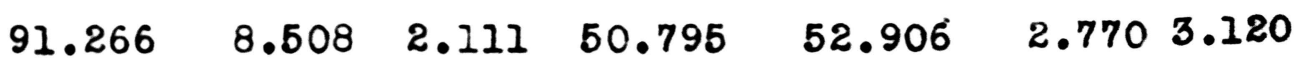

A comparison of Table XXI, digestible nutrients in 100 pounds of feods used in 1915-1916 test with Table III, digestible nutrients in 100 pounds of feeds used in 1914-1915 trial shows that the silage fed in the second trial was lower in dry matter, protein, nitrogen free extract, oarbohjdrates and fat than the silage fed in the first trial and was higher in crude fiber and ash.

In 100 pounds of timothy hay fed during 1915-1916 there was 1088 digestible protein, fiber, fat and ash than in the first 
trial. It contained slightly more nitrogen free extract and carbohydrates.

There was slightlg more digestible protein, nitrogen freo extract, fat and ash, and slightly loss fiber and carbohydrates in the alfalfa hay led during the second test.

The corn fed during the 1915-1916 trial was slightly lower in protein and slightly higher in dry matter, fiber, nitrogen free extract, carbohydrates, fat and ash than the corn fed during the first trial.

1 comparison of the oats fed during the second trial with those fed during the previous test shows that they were slightly lower in protein and ash. They were slightly higher in digestible fiber, nitrogen free extract, carbohydrates and fat.

The bran fed during the 1915-1916 trial was slightly lower in digestible protein, fiber and fat, and higher in nitrogen free extract and carbohydrates than that fed in 1914-1915. 
DESCRIPIION OF HORSES IN TEE 1915-1916 TEST

IOT I

IOT II

NANE AID IUUABRR AGE BREED NAMR AND ITUABER AGB BREED

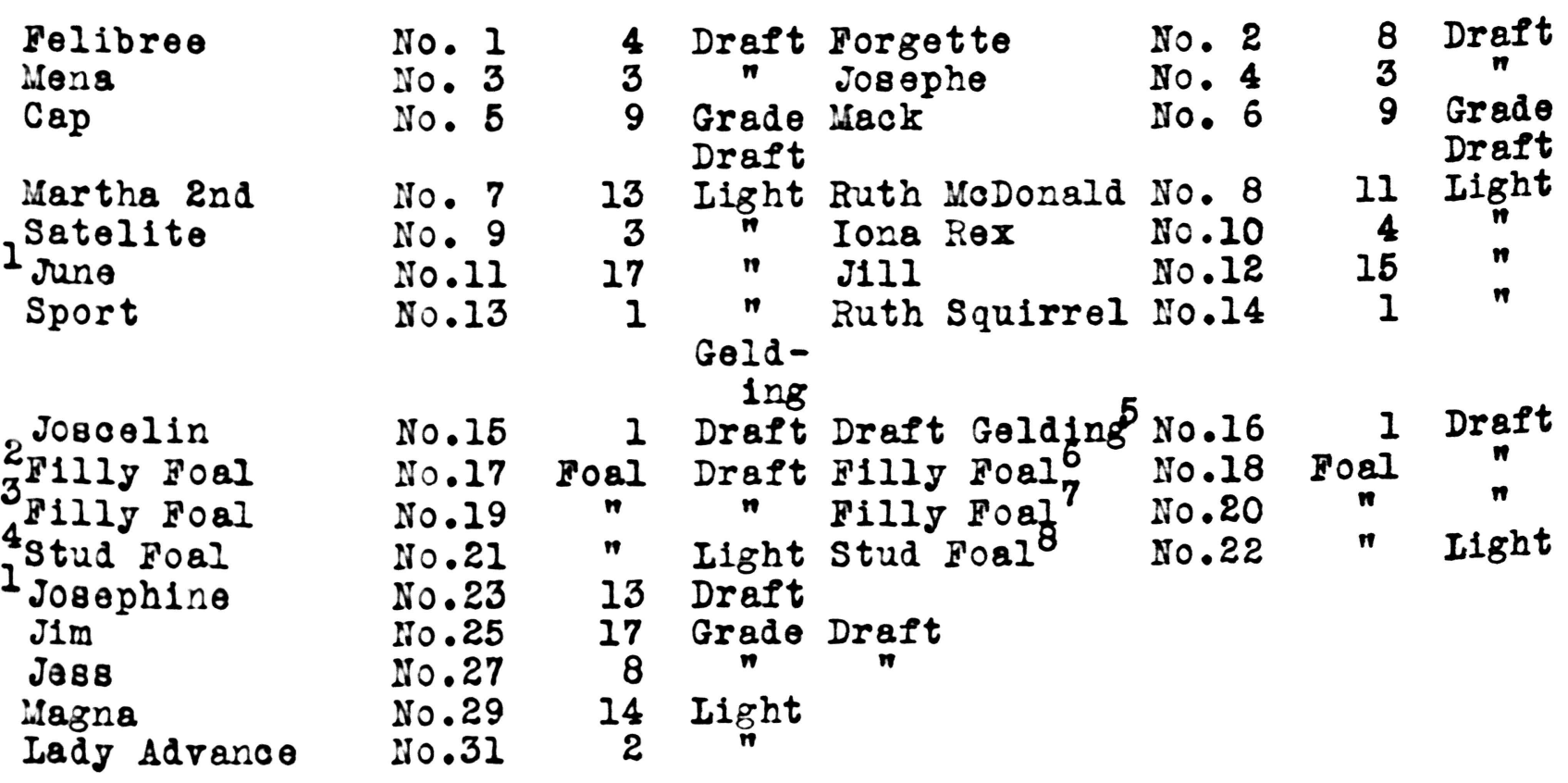

I Bred late in fall

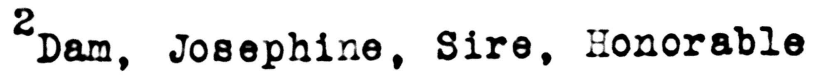

3 Dem, Felibree, Sire, Honorable

4Dam, Ruth MoDonald, Sire, Astral King

5arn, Finette, Sire Garainer

6am, Forgette, Sire, Honorable

7 Dam, Cervera, Sire, Honorable

8am, Iona Rex, Sire, Kentucky's Best 


\section{MANAGEENT DURIIIG SULER AND FALI PREVIOUS TO BEGINIING THE TEST \\ Horses numbered, $3,4,5,6,25$ and 27 were on dry} feed sumer and $f a l l$ and worked hard during that period. Number 11 and No. 31 were on dry feed. Number 11 was worked some and No. 31 driven a little.

Numbers 1, 2 and 23 were on pasture two months. They were brought in and put on dry feed september 5, and put at work. Number 23 ran a nail in her foot and was lame when the test began.

Numbers $8,10,14,16,18,20,22,5,7,15,17,19$, 21 and 29 were on pasture until December 10, except for a short time when the colts were weaned.

Number 12 was on dry feed and worked little during the summer.

\section{FEED RECEITED}

Tables XXII, XXIII, XXIV, XXV, XXVI, XXVII, XXVIII, XXIX, $200 X, X 0 X I, X 0 X I I, X X X I I I, X X X I V$ and $X X X V, g i v e$ the quantity of feed consumed. work performed and weights of each individual by weokly periods. In addition the total and average daily consumption of feed are shown. These data are presented for woekly periods in order that variations in the amount of feed consumed and in weight may be noted. The "silage fed horses" consumed per day an average of 3.804 pounds of silage in the oase of draft foal No. 19 to 15.908 pounds of silage in case of the saddle brood mare, Satelite No. 9. 
The sadde brood mare, Satelite No. 9, and the grade draft gelding, Jim No. 25, not at work, were fed a ration consisting of grain and silage only during the last five weeks of the test. No 111 effects were apparent.

\section{DIGESTIBIE NUTRIENTS RECEI VED}

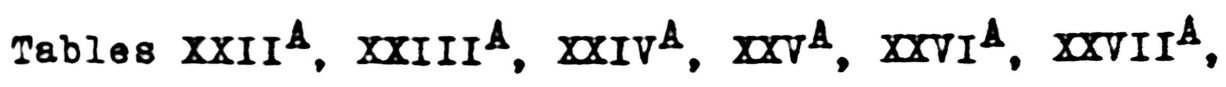

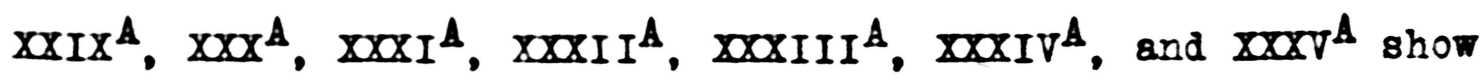
the total digestible nutrients received and the average digestible nutrients led daily. They also present the total digestible nutrients consumed and the average digestible nutrients received daily per 1,000 pounds live weight of the horses. This was based on the average weight of each horse thru the test. 
TABIE XXII

GROUP A

LATURE IDIE HORSES AIT INULES

b. "Open mares"*

Lot I June, No. 11, Standard bred mare

Lot II J111, No. 12, Standard bred mare

FEED CONSUAED AND WEIGH ${ }^{* *}$ BY WEEKLY PERIODS

LOM I

DATE

Deo. 17

" 2428

" 31

1916

Jan. 728

" 1428

" 2128

" $28 \quad 28$

$\mathrm{Feb}, 428$

n 1128

n 1828

n $25 \quad 28$

Woh. 328

Moh. 1028

Total

336

4.

5.

8.833

Dail
Ave.

\section{$z_{\text {HAY }}$}

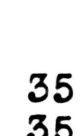

35

35

35

35

35

35

35

35

35

35

35

420

742

47.5

56.

70.

69.

67 .

70.

77 .

77 .

77 .

77 .
$3_{1030}$

1040

1040

1015

1020

1030

1020

1025

1040

1020

1020

1040

1012

${ }^{4} 18$

$336 \quad 1218$

4. $\quad 14.5$
IOT II

2 HAY WEIGHT

31010

$28 \quad 70 \quad 1035$

$\begin{array}{lll}28 & 91 & 1040\end{array}$

$\begin{array}{lll}28 & 91 & 1020\end{array}$

$28 \quad 91 \quad 1020$

$28 \quad 91 \quad 1040$

$28 \quad 112 \quad 1030$

$28 \quad 112 \quad 1025$

$28 \quad 112 \quad 1050$

$28 \quad 112 \quad 1030$

$28 \quad 112 \quad 1020$

$28 \quad 112 \quad 1050$

$28 \quad 112 \quad 1028$

$5_{18}$

* Not in foal

${ }^{1}$ Corn, oats, Bran, $2-2-1$

${ }^{2}$ alfalfa hay

3

Initial woight

${ }^{4}$ Los8 in weight

5

Gain in weight 
TABLE XXIIA

IOT I

DigesaIbIE NUTRIENTS CONSUME

TOMAI FEED

COISURED

DRY MATTER PROTEIN

CARBO-

$\frac{1}{2}$ Grain
Silage
Total
Ar. Da1ly

336.0
420.0
742.0

303.672

28.763

HYDRATES

FAT ASH

391.301

$\begin{array}{lll}177.378 & 9.342 \quad 10.758\end{array}$

264.456

44.650

$166.459 \quad 1.693$

38.338

959.429

11.731

150.381

8.288

14.558

11.422

85.144

$494.218 \quad 19.323 \quad 63.654$

1.014

$5.884 \quad .230$

.758

DIGESTIBIE NUMRIENTS CONSUMED*

PER 1,000 POUIDS LIVE WEIGHT

Total

934.205

82.906

481.22518 .815

61.981

Av. Daily

11.122

.987

$5.729 \quad .224$

.738

\section{LOT II}

DIGESTIBLE NUTRIENTS CONSURAR*

${ }_{2 \text { Gaj }}^{\text {IGain }}$

Total

Av. Daily
336.0
1218.0

303.672

1134.774

1438.446

17.124
28.763
129.486

158.249

1.884 $\begin{array}{lll}177.378 & 9.342 \quad 10.758\end{array}$

$482.730 \quad 4.897 \quad 111.179$

$660.108 \quad 14.239121 .937$

$7.858 \quad .170 \quad 1.452$

DIGESTIBLE NUTRIENTS CONSUMED*

PER 1,000 POUNDS IIVE WEIGHT

Total

$1395.189 \quad 153.491$

640.26013 .801118 .270

Av. Daily

16.609

1.826

$7.622 \quad .164 \quad 1.408$

${ }_{\text {corn, oats, bran, } 2-2-1}$

2

Alfalfa

*Pounds 
PHOTOGRAPHED AT THE BEGININING OF THE EXPERTIENT, DECEMBER 18, 1915 LOT II JILI, NUMBER 12 IOT I JUNE, ITUMBER 11

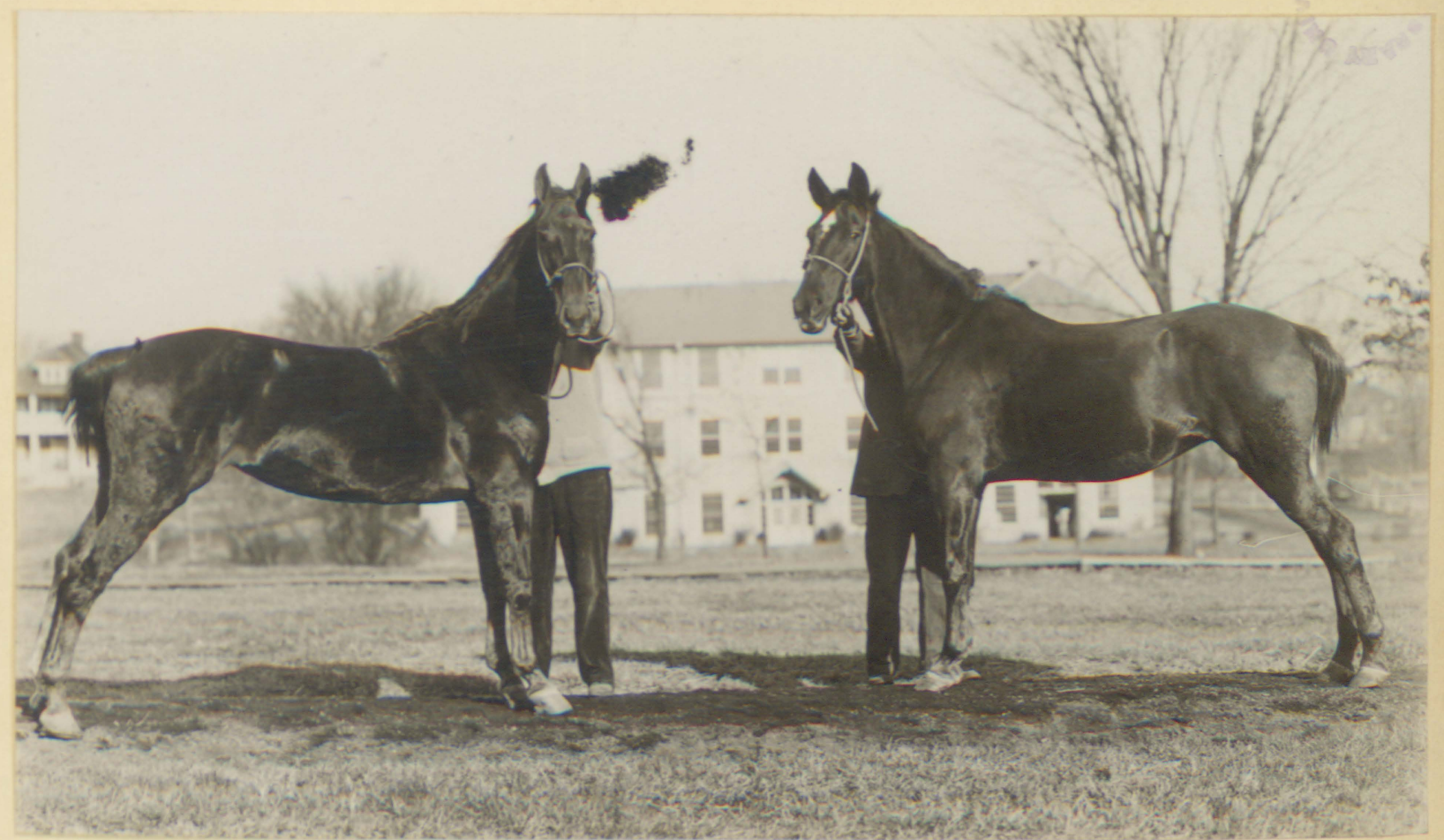

PHOTOGRAPHED AT THE CLOSE OF THE EXPERIMENT, MARCH 11, 1916

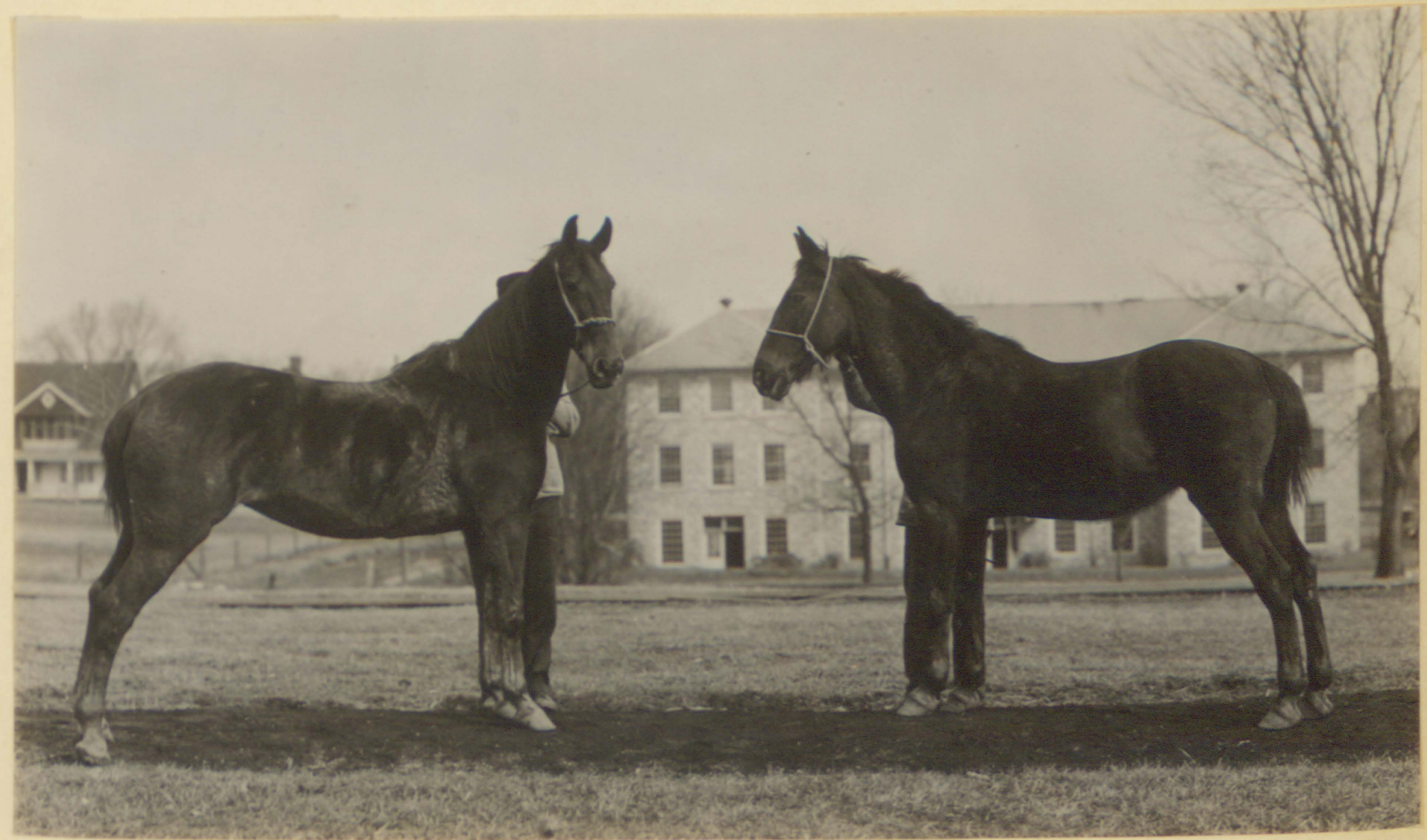


Tables XXII and XXII present the data for Group A-b, Lots I and II, mature lale open mares, thene No. II and $\mathrm{JIII}$ No. 12. Lot I receired silage as a part ration. Lot II was fed no 8ilage. Both lots were fed grain and alfalfa hay. These mares were in medium condition of flesh at the beginning of the experiment. The coat of hair of Jill, No. 12, indicated that she was in slightlg better thrift than June, No. 11. Neither mare rad suckled a foal the previous summer.

Lot I 108 t 18 pounds and Lot II gained 18 pounds during the test. Both lots consumed the same quantity of grain. Lot II receired 9.5 pounds of hay more per day than Lot $I$. Lot I consumed an average of 8.833 pounds of silage deily.

Table XXII shows that Lot II received da1ly 5.702 pounds of dry matter, .87 pounds of protein, 1.974 pounds of carbohydrates and .694 pounds of ash more than Lot I. Lot I was fed .06 pounds of fat per day more than Lot II.

These facts hold true when reduced to a thousand pound basis for the reason that the lots did not differ greatlg in woights.

At the close of the experiment these lots were in medium condition. There was no perceptible difference in their condition of flesh or thrift. 


\section{TABLE XXIII}

\section{GROUP A}

MATURE IDIE HORSES AND MULES

c. Pregnant mares

Lot I Martha 2nd, No. 7, Registered sadale mare

Lot II Ruth MoDonald, No. 8, Registered sadale mare FEED CONSUEE ${ }^{*}$ AND WEIGHT* BY WEEKLY PERIODS

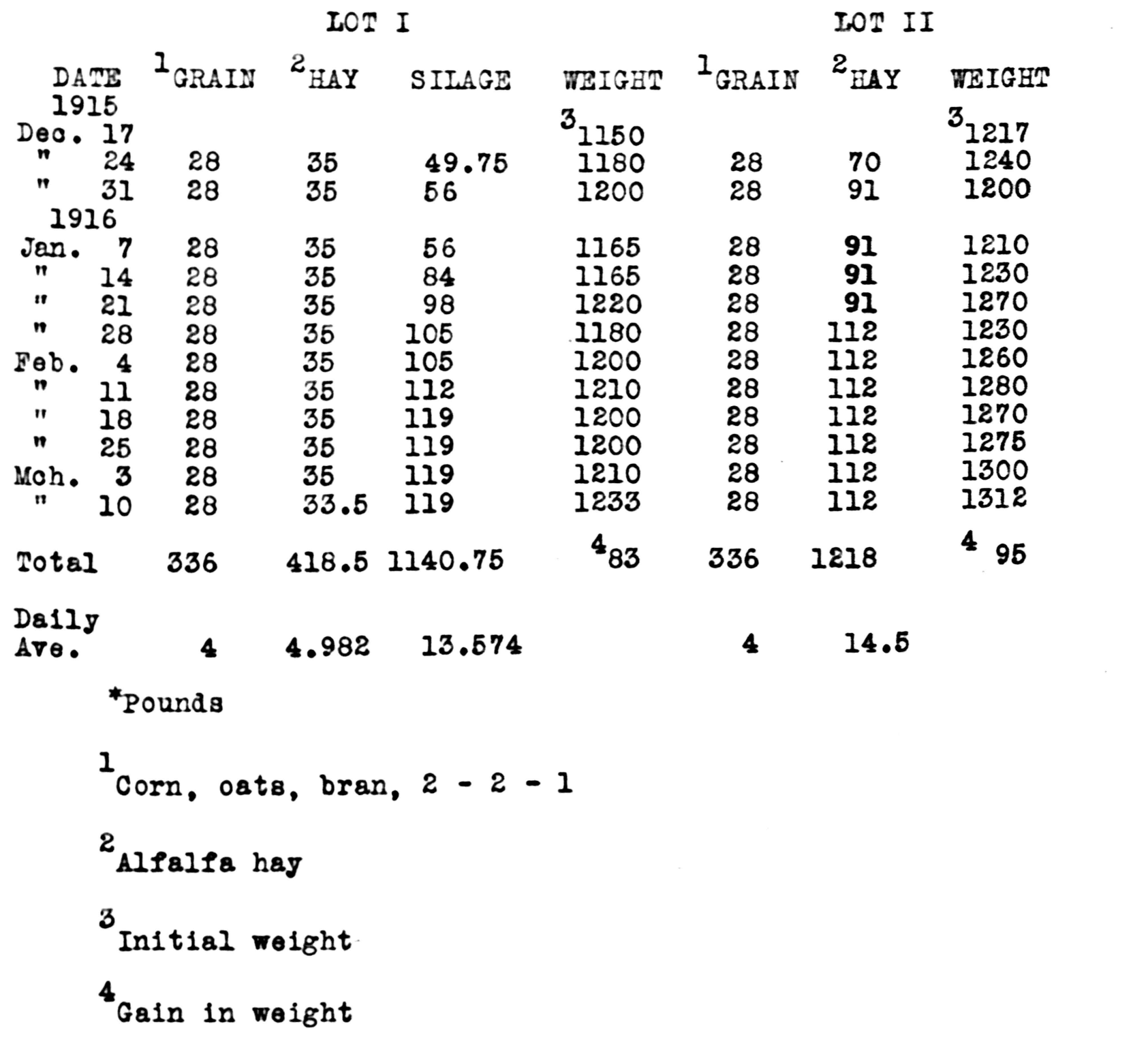


TABIR XXIII

LOM I

DIGESTIBLE NUTRIEN $M$ COMSUIED*

TOTAL FEED CONSUNED

$1_{\text {Grain }}$
$2_{\text {Slage }}$

Total

Av. Daily

\section{0 \\ 418.5 \\ 1140.25}

DRY LATTER PRC 303.672 389.904 406.397

1099.973

13.095
CARBOPROTEIN HYDRATES FAT ASH $\begin{array}{llll}28.763 & 177.378 & 9.342 & 10.758\end{array}$ $44.491 \quad 125.864 \quad 1.687 \quad 38.201$ $18.027 \quad 231.094 \quad 12.737 \quad 22.372$

$91.281 \quad 534.336 \quad 23.766 \quad 71.331$ $\begin{array}{llll}1.087 & 6.361 & .283 & .849\end{array}$

DIGESTIBLS MUTRIENTS CONSUNRE*

PER 1,000 POUNDS IIVE WEIGHT

sotal

Av. Da1ly
922.023

10.976
76.514

.911
447.89319 .921

$5.332 \quad .237$
59.791

.712

\section{IOS II}

\section{DIGESTIBIE NUTRIENTS CONSUNED*}

\begin{tabular}{|c|c|c|c|c|c|c|}
\hline $\begin{array}{l}\mathrm{I}_{\text {Grain }} \\
\mathrm{z}_{\text {Eay }}\end{array}$ & $\begin{array}{r}336.0 \\
1218.0\end{array}$ & $\begin{array}{r}303.672 \\
1134.774\end{array}$ & $\begin{array}{r}28.763 \\
129.486\end{array}$ & $\begin{array}{l}177.378 \\
482.730\end{array}$ & $\begin{array}{l}9.342 \\
4.897\end{array}$ & $\begin{array}{r}10.758 \\
111.179\end{array}$ \\
\hline Total & & 1438.446 & 158.249 & 660.108 & 14.239 & 121.937 \\
\hline Av. Daily & & 17.124 & 1.884 & 7.858 & .170 & 1.452 \\
\hline
\end{tabular}

DIGESTIBLE HUTRIENMS COUSURED*

PER 1,000 POUNDS IIVE WEIGHT

Total

Av. Daily
$1147.997 \quad 126.296$

13.667
$526.822 \quad 11.364 \quad 97.316$ $6.296 \quad .135 \quad 1.159$

$1_{\text {Corn, oats, bran, } 2-2-1}$

2

Alfalfa

*Pounds 
GROUP A $=0-1$

PHOTOGRAPHED AT THE BEGINIING OF FXXPERTMEITT, DECHIBER 18, 1915 LOT II RUTH MODONAID, ITUMBER 8 IOT I MARTHA 2ND, NUMBER 7

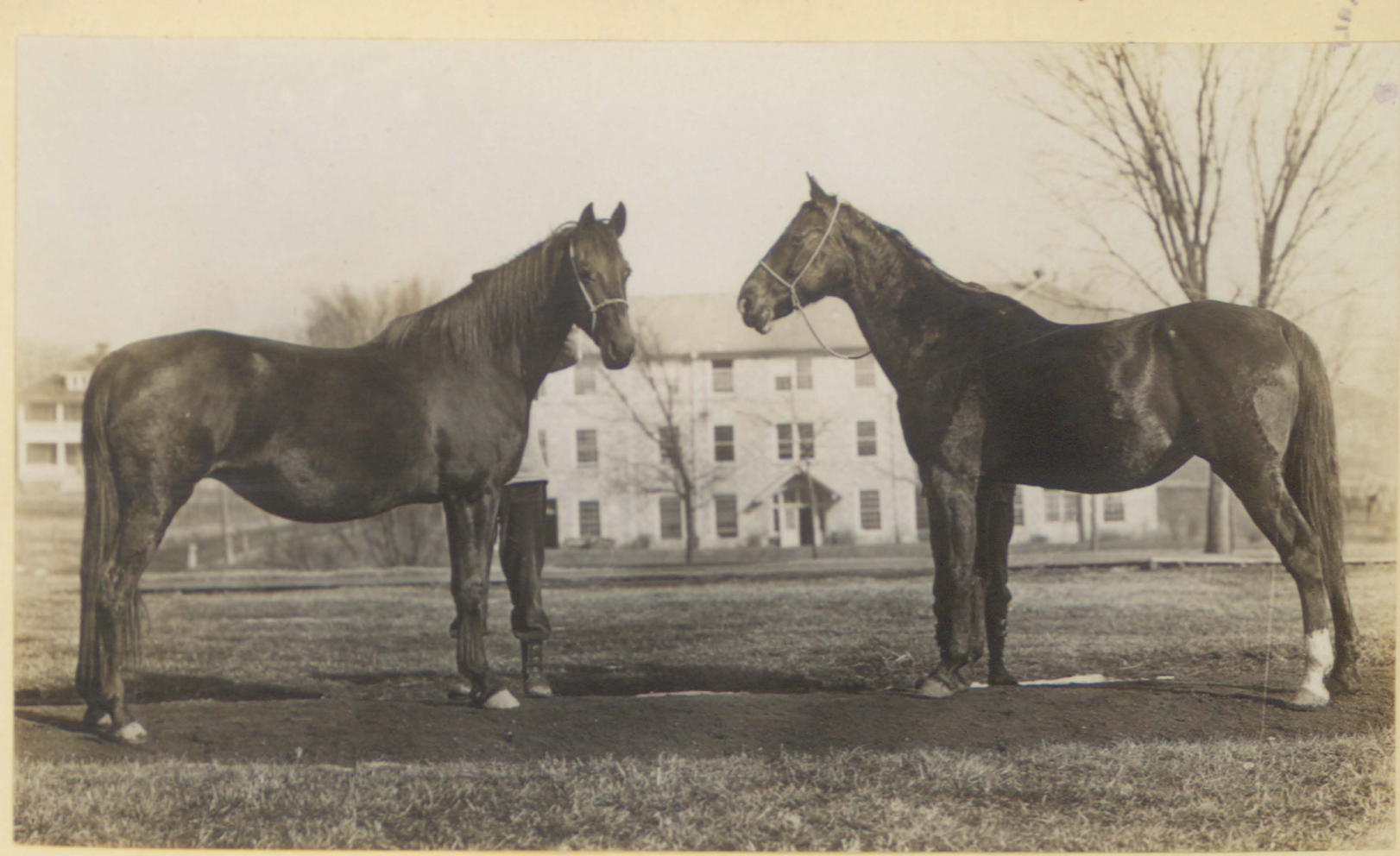

PHOTOGRAPHED AT THE CLOSE OF THE EXPERIMENT, MARCH 11, 1916

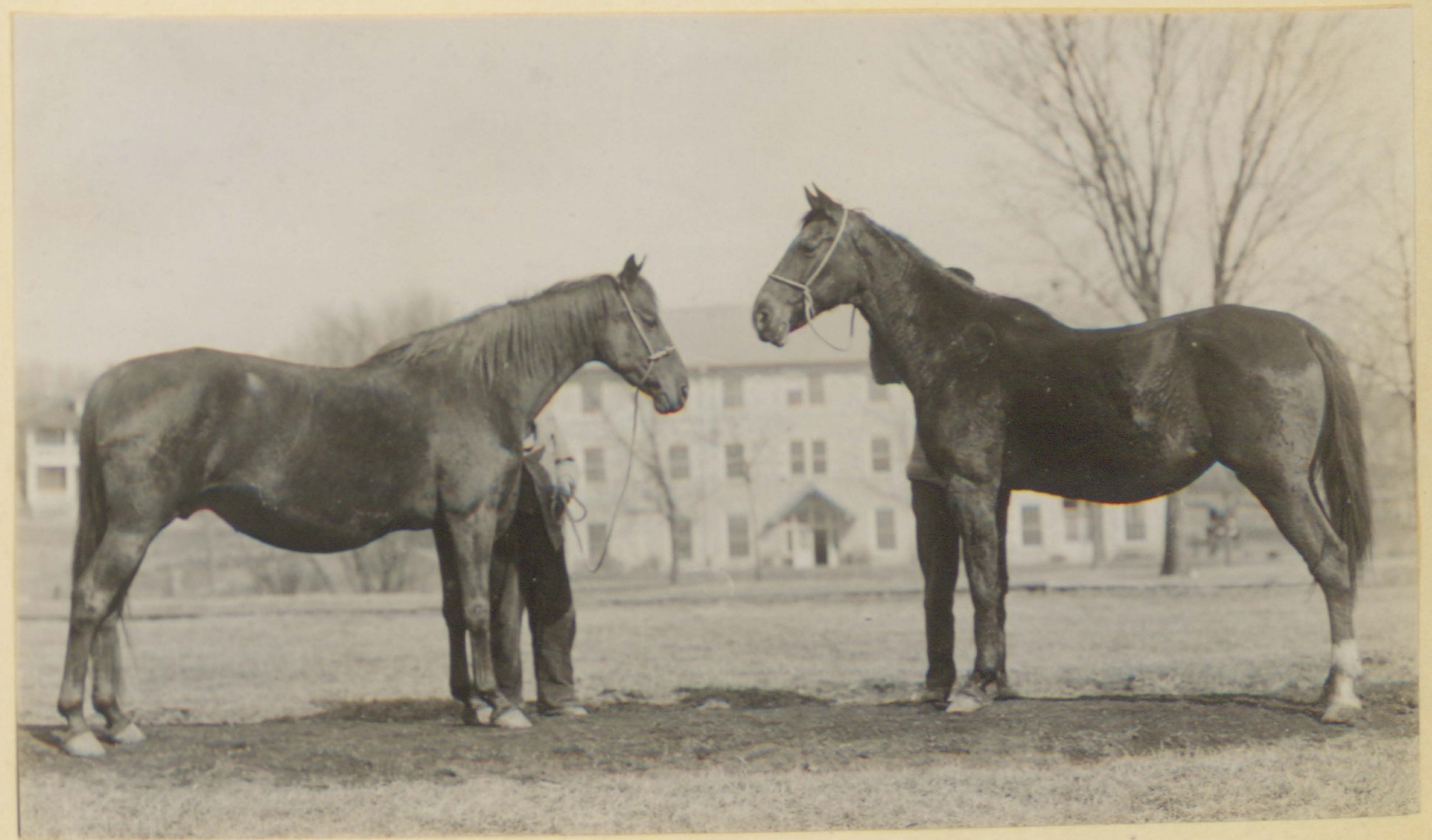


Tab es XXIII and XXIII ${ }^{A}$ present the date for Group B-c-l, Iots I and II, mature idle pregnant mares, Nartha $2 n d$, No. 7 and Rutr icjoneld, No. 8. Lot I received silage as a part ration, Lot II received no sil goe. Both lots were fed grain and alfalfa hay.

Ware No. 8 was in better condition than Ware No. 7 at the beginning of the test. Marthe 2nd, No. 7, was Iame when the trial began. The initial weight of Iot I was 1150 pounds and the initiel weight of Lot II was 1217 pounds.

These mares produced foals in the spring of 1915 and suckled their foals until september 10, 1925. Mare No. 7 was bred June 15, 1915 and mare No. 8 was bred April 18, 1915.

Lot I gained 83 pounds and Lot II gained 95 pounds during the twolve week feeding period. Both lots received the same amount of grain. Lot II was feà daily 9.52 pounds of hay more than Lot I. Lot I consumed an average of 13.574 pounds of silage per day.

Referring to the table of digestible nutrients, it will be noted that Lot II received per day an average of 4.029 pounds of dry matter, .797 pounds of protein, 1.497 pounds of carbohydrates and .603 pounds of ash more than Iot I. Iot I consumed .112 pounds of fat per day more than Iot II.

The table of digestible nutrients consumed per thousand pounds Iive weight shows that Lot II was fed daily 2.691 pounds of dry metter, .593 pounds of protein, .964 pounds of oarbohydrates, and .447 pounds of ash more than Iot I. Lot I re- 
coired .I02 pounds of fat per day more than Iot II. Lot II was still in botter condition at the close of the experiment than Lot $I$. Her hair and skin indicated slightly better thrift. 


\section{TABLE XXIV}

GROUP A

MATURE IDLE HORSES AND NULES

- - 2 Pregnant mares

Lot I Satelite, No. 9, Saddie bred mare

Lot II Iona Rex, No. io, Registered sadde mare

FEED CONSUIED AND WEIGHTS* BY WEEKCL PERIODS

IOT I

IOT II

DATE $1_{\text {GRAIN }} 2_{\text {HAY }}$ SILAGE WEIGHT $1_{\text {GRAIN }} 2_{\text {HAY }}$ MEIGH

\section{5}

Dec. 17

" 2428

" $31 \quad 28$ 1916

\section{Jan. 728}

" $14 \quad 28$

n 2128

n $28 \quad 28$

Feb. 428

n 1128

n 1828

n $25 \quad 28$

Moh. 328

" 1028

Total

$$
336
$$

4

Dally

Are.
$5_{245} \quad 1336.25$

$5_{5}$

15.908

35

35

35

35

35

35

35

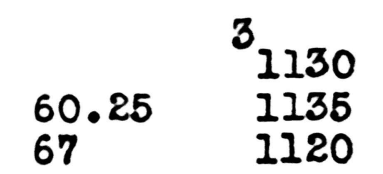

70

84

98

105

105

140

161

159

155.5

131.5

${ }^{1}$ corn, oats, bran, $2-2-1$

${ }^{2}$ Alfalfa hay

3

Initial weight

4 I088 in weight

Fod hay only soven weoks

6

${ }^{6}$ Gain in weight

*Pounds 


\section{TABLE XXIVA \\ LOM I}

DIGESTIBIE NUTRIENMS CONSUIED*

TOMAI FEDD DRY WATTER PROTEIN CARBOCOISUNED

HYDRATES FAT ASH

\begin{tabular}{|c|c|c|c|c|c|c|}
\hline $\begin{array}{l}{ }_{2}^{1} \text { Grain } \\
\text { Haj } \\
\text { S1lage }\end{array}$ & $\begin{aligned} 336.0 \\
245.0 \\
1336.25\end{aligned}$ & $\begin{array}{l}303.672 \\
228.259 \\
476.253\end{array}$ & $\begin{array}{l}28.763 \\
26.046 \\
21.126\end{array}$ & $\begin{array}{r}177.378 \\
97.101 \\
270.818\end{array}$ & $\begin{array}{r}9.342 \\
.987 \\
14.926\end{array}$ & $\begin{array}{l}10.758 \\
22.364 \\
26.217\end{array}$ \\
\hline Total & & 1008.184 & 75.935 & 545.297 & 25.255 & 59.339 \\
\hline Av. Daily & & 12.002 & .904 & 6.492 & .265 & .706 \\
\hline \multicolumn{7}{|c|}{$\begin{array}{l}\text { DIGESTIBLE NUTRIENTS CONSUNED* } \\
\text { PER } 1,000 \text { POUIDS IIVE WEIGHA }\end{array}$} \\
\hline Total & & 900.969 & 67.860 & 487.307 & 22.569 & 53.029 \\
\hline Av. Dally & & 10.726 & .808 & 5.801 & .269 & .631 \\
\hline
\end{tabular}

IOT II

$\log _{\text {Hay }}$

Total

Av. Dails
336.0

1215.0
303.672

1131.979

1435.651

17.091
28.763

129.167

157.930

1.880 $\begin{array}{lll}177.378 & 9.342 \quad 10.758\end{array}$ $481.541 \quad 4.896110 .905$

658.91914 .238121 .663

$7.844 \quad .170 \quad 1.448$
DIGESTIBLE NUMRIEITS CONSUUED*

PER 1,000 POUNDS IIVE WEIGHT
Total

Ar. Da11J
1339.226

15.943

147.323

$614.663 \quad 13.188 \quad 113.492$

1.754

${ }^{1}$ Corn, oats, bran, $2-2-1$

infalfa

*Pounds 


\section{GROUP A - $0-2$}

PHOTOGRAPHED AT THE BEGINIING OF EXPERTMENT, DECHMBER 18, 1915 IOT I SATELITE, NUMBER 9 IOT II IONA REX, NUMBER 10

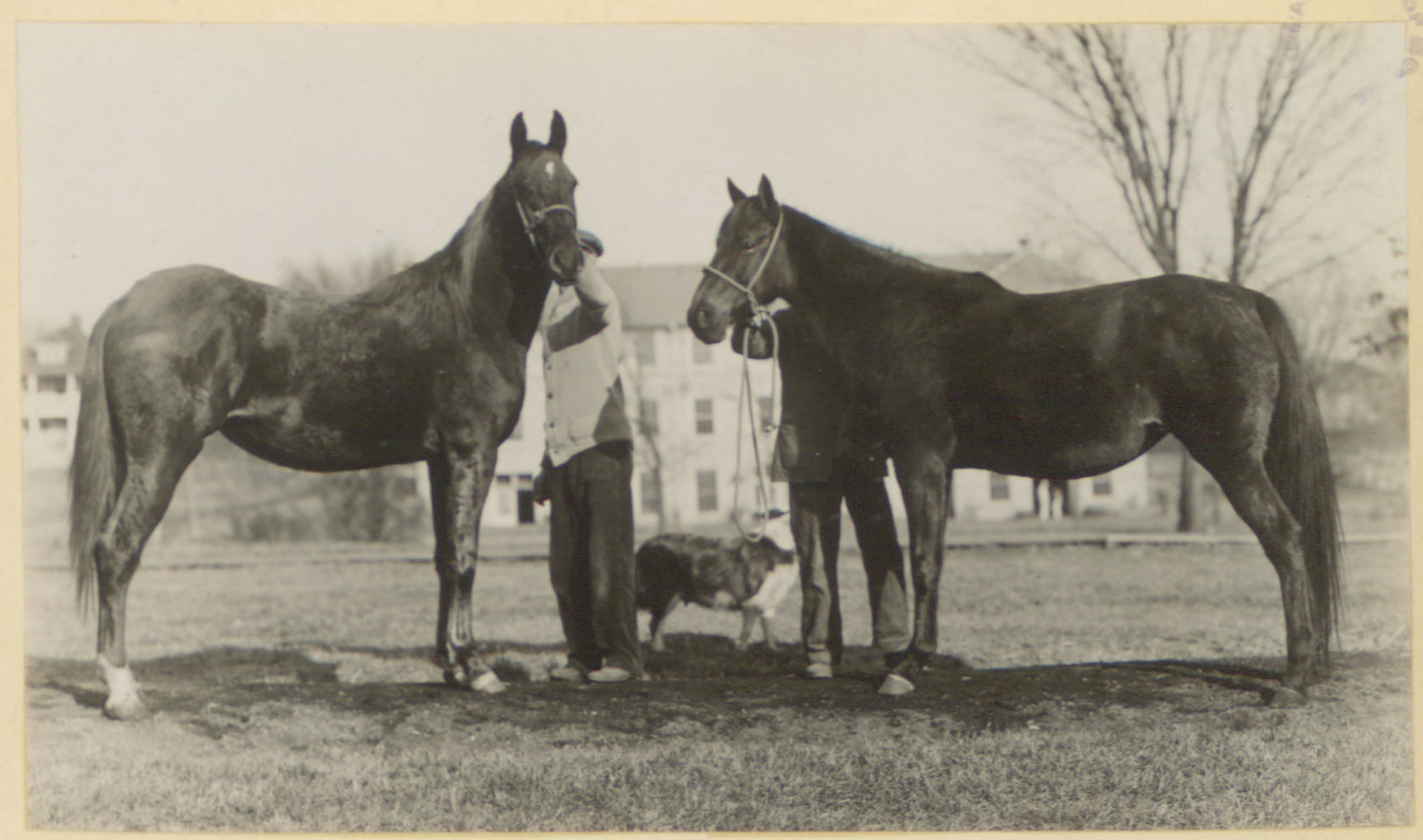

PHOTOGRAPHED AT THE CLOSE OF THE EXPERIMENT, MARCH 11, 1916

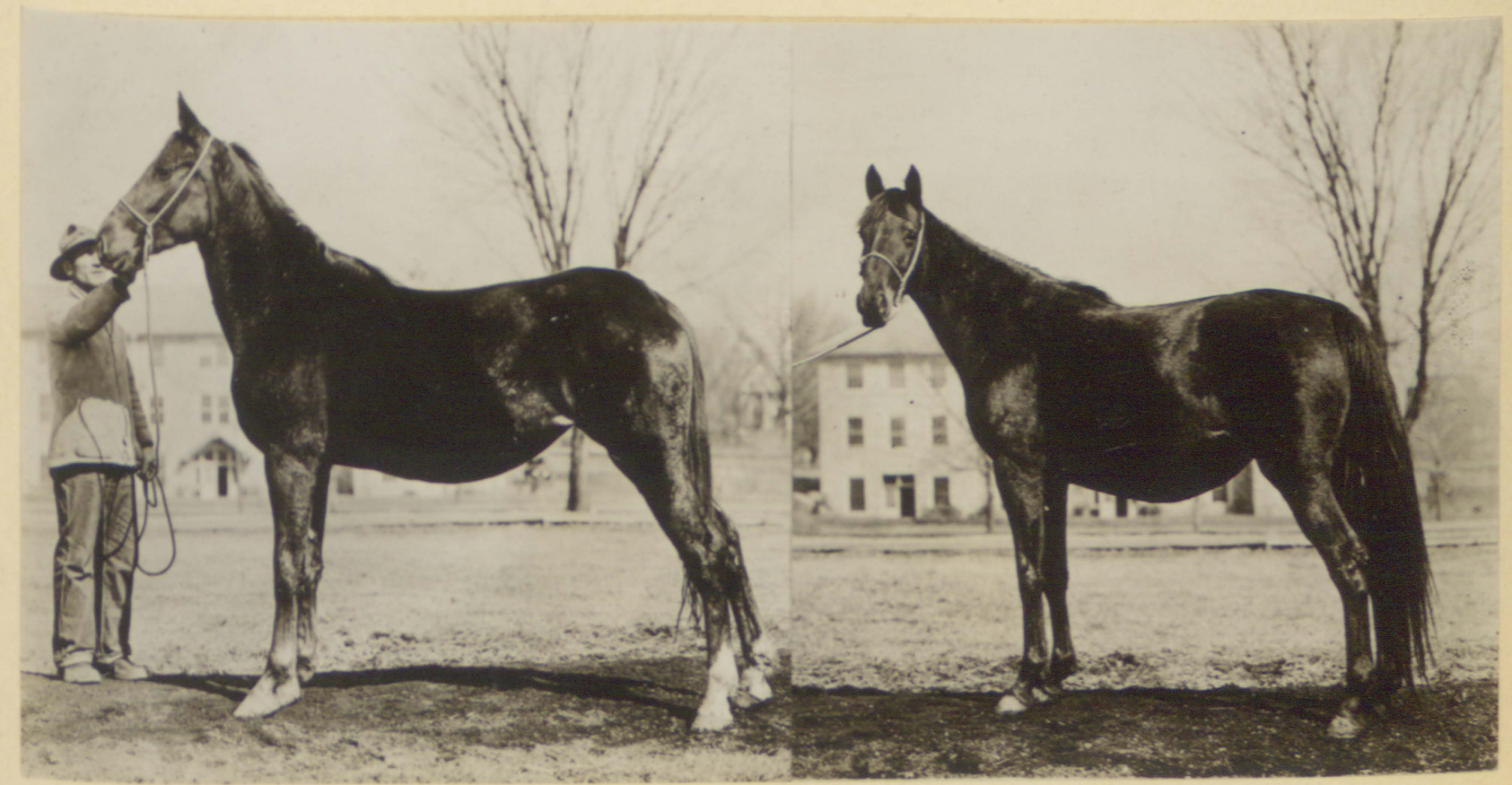

MATURE IDLE PREGINANT MARES 
Tables $X X I V$ and $X X I \nabla^{A}$ present the date for Group A-0-2, Lots I and II, mature idle pregnant mares, Satelite, No. 9 and Iona Rex, No. 10. Lot I receired silege as a part ration. Iot II was fod no sllage. Both lots were fed grain and alfalfa hay.

Lare No. 9 was 97 pounds heavier than mare No. 10 at the beglnning of the test. She was a taller, larger framed mare, not so compactly built. The initial weight of satelite, No. 9 was 1130 pounds and the initial weight of Iona Rex, No. 10 was 1033 pounds.

These mares produced foals in the spring of 1915 and suckled their foals until september 10, 1975.

Mare No. 9 was bred May 29, 1915 and Mare No. 10 was bred April 18, 1915.

Lot I lost 3 pounds and Lot II gained 67 pounds during the test.

Both lots consumed the seme amount of grain. Iot I recel red 5 pounds of hay per day during the first seven weeks of the test. During the last fire weeks of the test this lot was led only silage and grain. Lot I consumed a daily average of 15.908 pounds of silage. Lot II was fed an average of 14.64 pounds of hay per day.

The toble of digestible nutrients consumed shows that Lot II received 5.089 pounds of dry matter, .976 pounds of protein, 1.352 pounds of carbohydrates and .742 pounds of ash more per day than Lot I. Lot I consumed an average of .095 
pounds of fat per day more than Lot II.

Then reduced to a thousand pound basis, Table XXIV shors that Lot II consumed daily 5.217 pounds of dry matter, .946 pounds of protein, 1.505 pounds of carbohydrates and .720 pounds of ash more than Lot I. Lot I was fed .112 pounds of fat per day more than Lot II.

When the experiment closed, Iot II was in better condition of flesh. The condition of the halr and skin of Lot I indicated that she was in better thrift than Iot II. Both lots were in medium breeding condition. 


\section{TABIE XXV}

GROUP B

MATURE HORSES AND LULES PERPORUIIIG AVRRAGE WINTER FARII TORK

b. Geldings

Lot I Cap, No. 5, Grade draft gelding

Lot II Hack, No. 6, Grade draft gelding

FEED CONSUUAR, HEIGHTS* ATD WORK HOURS BY HEEKLY PERIODS IOM I

IOI II

DATS ${ }^{1}$ GRAIN $2_{\text {HAY }}$ SILAGE WEIGHT $1_{\text {GRAIN }} 2_{\text {HAY WEIGHT }} 5_{\text {TORK }}$

\begin{tabular}{|c|c|c|c|c|c|c|c|c|c|}
\hline & & & & & & & & & \\
\hline 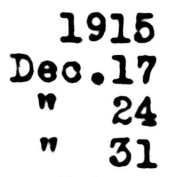 & $\begin{array}{l}100 \\
100\end{array}$ & $\begin{array}{l}61 \\
69\end{array}$ & $\begin{array}{l}56 \\
56\end{array}$ & $\begin{array}{r}{ }_{1310} \\
1300 \\
1260\end{array}$ & $\begin{array}{l}100 \\
100\end{array}$ & $\begin{array}{l}105 \\
104\end{array}$ & $\begin{array}{r}{ }_{1310} \\
1300 \\
1290\end{array}$ & $\begin{array}{l}41 \\
41\end{array}$ & B \\
\hline 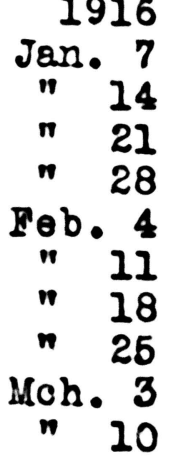 & $\begin{array}{l}100 \\
100 \\
100 \\
100 \\
100 \\
100 \\
100 \\
100 \\
100 \\
100\end{array}$ & $\begin{array}{l}70 \\
70 \\
70 \\
68 \\
70 \\
58.75 \\
46 \\
50 \\
56 \\
52.5\end{array}$ & $\begin{array}{l}56 \\
84 \\
98 \\
66.75 \\
105 \\
112 \\
119 \\
119 \\
117 \\
119\end{array}$ & $\begin{array}{l}1265 \\
1260 \\
1270 \\
1260 \\
1275 \\
1230 \\
1260 \\
1280 \\
1280 \\
1280\end{array}$ & $\begin{array}{l}100 \\
100 \\
100 \\
100 \\
100 \\
100 \\
111 \\
120 \\
120 \\
120\end{array}$ & $\begin{array}{l}105 \\
105 \\
105 \\
124.5 \\
126 \\
126 \\
117 \\
120 \\
126 \\
123\end{array}$ & $\begin{array}{l}1285 \\
1300 \\
1300 \\
1280 \\
1290 \\
1250 \\
1260 \\
1250 \\
1290 \\
1272\end{array}$ & $\begin{array}{l}22 \\
21 \\
32 \\
45 \\
41 \\
43 \\
56 \\
37 \\
52 \\
55\end{array}$ & $\begin{array}{r}10 \\
5 \\
3\end{array}$ \\
\hline Total & 1200 & 741.25 & 21237.75 & $4_{30}$ & 1271 & 1386.5 & & 486 & 8 \\
\hline $\begin{array}{l}\text { ailg } \\
\text { ve. }\end{array}$ & 14.286 & 8.824 & 13.545 & & 15.131 & 16.506 & & & \\
\hline
\end{tabular}

${ }^{1}$ corn, oats, bran, $2-2-1$

2Timothy hay

3 Initial weight

4088 in weight

B - heary work, B - medium and light work 


\section{TABIE XON \\ IOT I}

DIGESTIBIE NUMRIENTS CONSUAED*

TOTAL PEED

CONSURARD

DRY LATTER PROTEITI

CARBO-

IGrain

2 Hay

1200.0
741.25
1137.75

1094.977

102.726

7.323

692.009

17.988

63.5 .507

275.545

230.588

PAT

ASH

Total

Ar. Da1ly

2192.491

128.0371139 .640

33.140

7.023

12.709

38.420

35.654

22.323

26.101

1.524

13.567

52.872

96.397

DIGESTIBLE NUTRIENTS CONSUNAED*

PER 1,000 POUNDS IIVS WRIGHT

Total

1723.656

100.658

895.943

41.566

75.784

Ar. Dailg

20.519

1.198

10.666

.495

.902

\section{IOT II}

DIGESTIBLE NUMRIZNTS CONSUNRD*

$\begin{array}{ll}I_{\text {Grain }} & 1271.0 \\ 2_{\text {Hag }} & 1386.5\end{array}$

Total

Ar. Daily
1159.747

1294.395

2454.142

29.216
$108.767 \quad 671.070$

13.684518 .404

$35.332 \quad 40.635$

13.00566 .691

122.4511186 .474

1.458

14.125
48.337107 .326

$.575 \quad 1.278$

DIGSSTIBLE NUTRIENTS CONSURAB*

PER 1,000 POUNDS LI VE WEIGHT

Total

Av. Daily
1905.021

22.679

191.438

924.765

37.675

83.652

$1.136 \quad 12.009$

.449 .996

*Pounds

${ }^{2}$ Corn, oats, bran, $2-2-1$

E Timothy 
GROUP $B-b$

PHOTOGRAPHED AT THE BEGINNING OF EXPERIMENT, DECEIBER 18, 1915 IOT I CAP, NUMBER 5

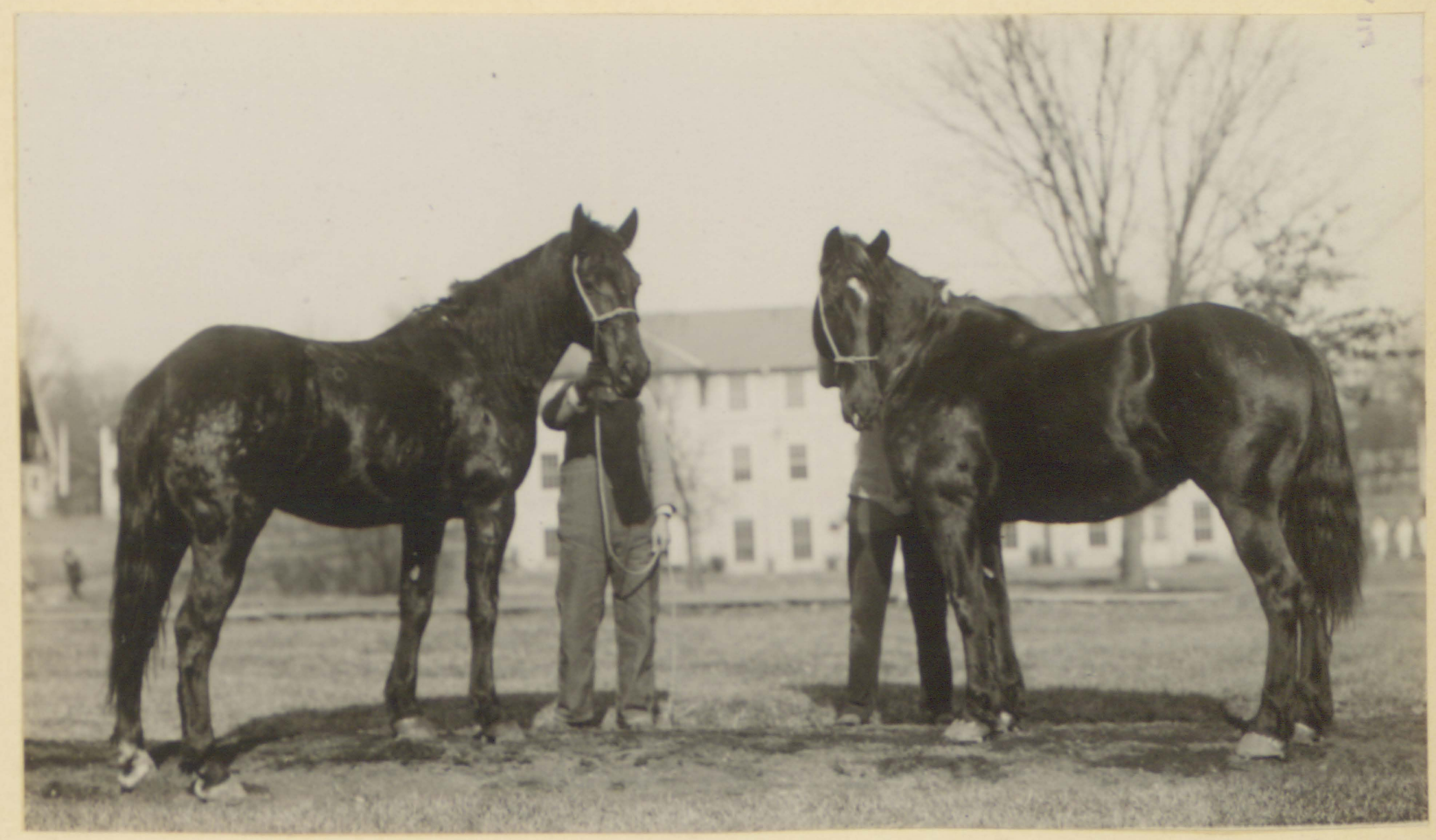

PHOTOGRAPHED AT THE CLOSE OF THE EXPERIMENT, MARCH 11, 1916 IOT II MACK, NUMBER 6 IOT I CAP, NUMBER 5

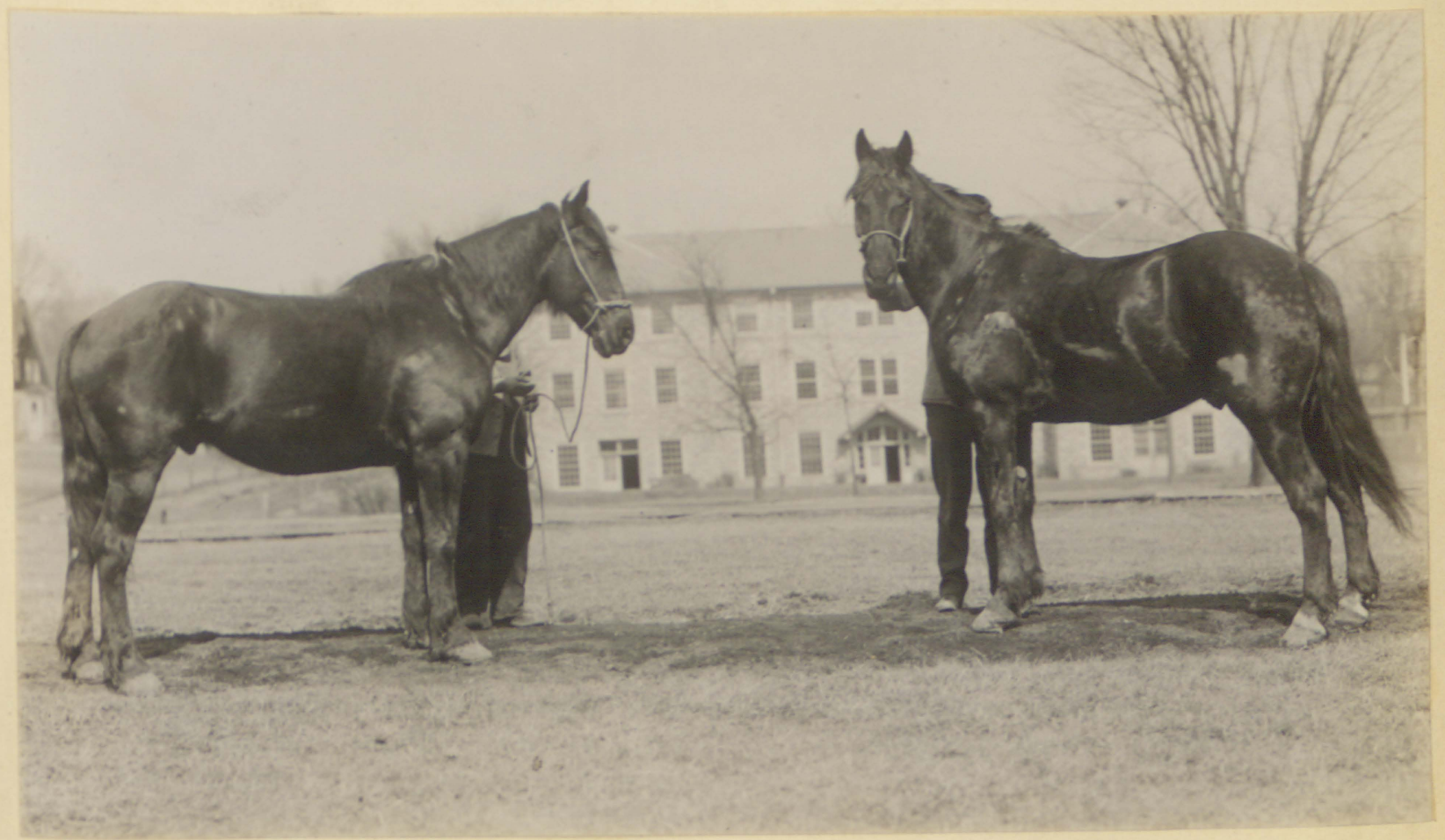

MATURE GELDINGS PERFORMING AVERAGE WINTER FARM WORK IOT I, NO. 5, SILAGE LOT II,NO. 6 , NO SILAGE 
Tables $X X V$ and $X X V^{A}$ present the data for Group B-b Lots I and II, mature horses performing average winter farm work, Cap, No. 5 and Wack, No. 6. Lot I received silage as a part ration. Lot II was fed no silage. Both lots were fed grain and timothy hay.

These horses were in medium condition of flesh at the beginning of the experiment. Cap, No. 5 was in slightly better condition than lack, No. 6. Their initial weights were the same. This team performed 486 hours of heary labor and 18 hours of medium and light labor during the test. Lot I lost 30 pounds and Lot II lost 28 pounds during the twelve week leoding period.

Lot II receited .845 pounds of grain and 7.682 pounds of hay more per day than Lot I. Lot I consumed an average of 13.545 pounds of sllage per day.

The table of digestible nutrients shows that Lot II received 3.115 pounds of dry matter, .558 pounds of carbohydrates, .054 pounds of fat more per day than Lot $I$. Iot I consumed daily .066 pounds of protein and .13 pounds of $\mathrm{ash}$ more than Lot II.

These faots hold true when reduced to a thousand pound basis for the reason that the lots did not differ greatly In weights during the test.

There was no perceptible difference in the condition or thrift of these lots at the close of the experiment. 
TABIE XXVI

GROUP B

LATURR HORSES AND WULES PERPORMING AVERAGE WINTER FARM WORK

c. Pregnant mares

Lot I Fellbree, No. 1, Registered Percheron mare

Lot II Forgette, No. 2, Registered Percheron mare

FER CONSUNRE; WORK HOURS AND WEIGHMS*BY WEZKLYY PERIODS

IOI I

IOT II

DATE $1_{\text {GRAIN }} 2_{\text {BAY }}$ SILAGB WEIGHT $I_{\text {GRAIN }} 2_{\text {HAY WEIGHT }} 5_{\text {TORK }}$

1915

Dec.17

" $24 \quad 100 \quad 70$

" $31 \quad 100 \quad 70$

70

$\begin{array}{lrrrrrr} & 3_{1533} & & & 3_{1630} & \text { A } & \text { B } \\ 54 & 1550 & 100 & 105 & 1660 & 20 & 12 \\ 53.5 & 1490 & 100 & 105 & 1620 & 38 & 6\end{array}$

1916

Jan. 7100

70

56

1530

$100 \quad 105$

$1620 \quad 33$

70

84

$100 \quad 105$

163020

" 212100

70

98

$100 \quad 105$

$1600 \quad 29 \quad 5$

$70 \quad 105$

1550

$100 \quad 126$

105

$100 \quad 126$

1615

1640

1630

$100 \quad 126$

161036

1545

$120 \quad 122.5$

162036

1520

120124.

165048

1490120126.

163745

6

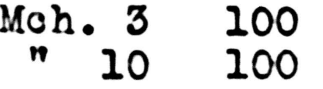

$54 \quad 119$

$4_{43} \quad 1271 \quad 1401.5$

$67443 \quad 44$

Total 1200

775.51130 .5

15.13116 .685

Daily

Ave.

$14.286 \quad 9.226 \quad 13.452$

*Pounds

${ }^{1}$ corn, osts, bran, $2-2-1$

2 Timothy hay

3

Initial woight

${ }^{4}$ Loss in weight

${ }^{5}$ A - heary work, B - medium and light work

${ }^{6}$ Gain in woight 
TABLE XXVI

IOT I

DIGESTIBLE NUMRINITS CONSURED*

TOMAL FEED

$1_{G r a i n}$

2 Hay

Sllage

Total

Av. Daily
CONS URCED

DRY NATMER PROTEIN

$1094.977 \quad 102.726$

$723.517 \quad 7.649$

$402.743 \quad 17.865$

775.5

1130.5
CARBO-

HYDRATES FAT ASH

$2221.237 \quad 128.240 \quad 1150.615 \quad 53.032 \quad 97.869$

26.443 $\begin{array}{lll}633.507 & 33.140 \quad 38.420\end{array}$

$288.091 \quad 7.270 \quad 37.278$

$229.017 \quad 12.622 \quad 22.171$

$\begin{array}{lll}13.698 \quad .631 & 1.165\end{array}$

DIGESTIBLE NUTRIZNTS CONSUIED*

PER 1,000 POUNDS LIVE WEIGET

Total

AT. Daily
1457.505

17.351

84.147

$754.997 \quad 34.798$

64.220

1.002

8.988

.414

.765

IOT II

DIGESTIBIE MUMRIENTS CONSUIED*

$I_{\text {Grain }}$
R Hay $_{\text {Hat }}$

Total

Av. Da1ly
1271.0

1401.5
$1159.747 \quad 108.767$

$1308.398 \quad 13.833$

$2468.145 \quad 122.600$

29.299

1.460
671.07035 .332

520.98013 .146

40.635

67.412

$1192.050 \quad 48.478 \quad 108.047$

14.191 .577

1.286

DIGESTIBLE NUMRIENTS CONSUMED*

PER 1,000 POUNDS LITE WEIGHT

Total

A7. Da115
1516.060

18.048

.897 $\begin{array}{lll}732.218 & 29.778 \quad 66.381\end{array}$

$8.717 \quad .355 \quad .790$

${ }^{1}$ Corn, 0ats, bran, $2-2-1$

2 Ilmothy

*Pounds 
PHOTOGRAPHISD AT THE BEGINIING OF EXPERIIENT, DECBMBER 18, 1915 IOT II FORGETIE, NUNBER 2

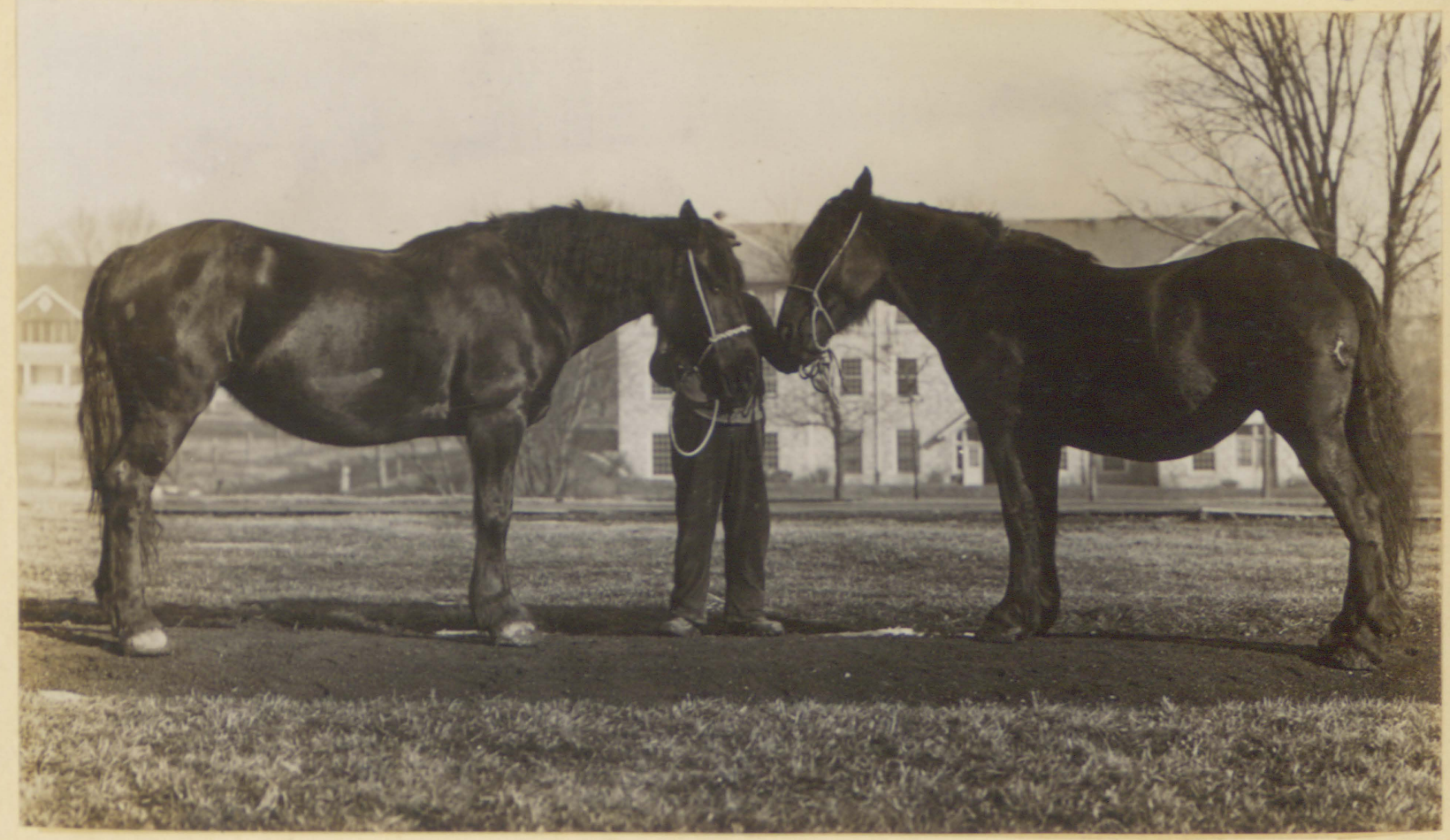

PHOTOGRAPHED AT THE CLOSE OF THE BXPERIMENT, MARCH 11, 1916

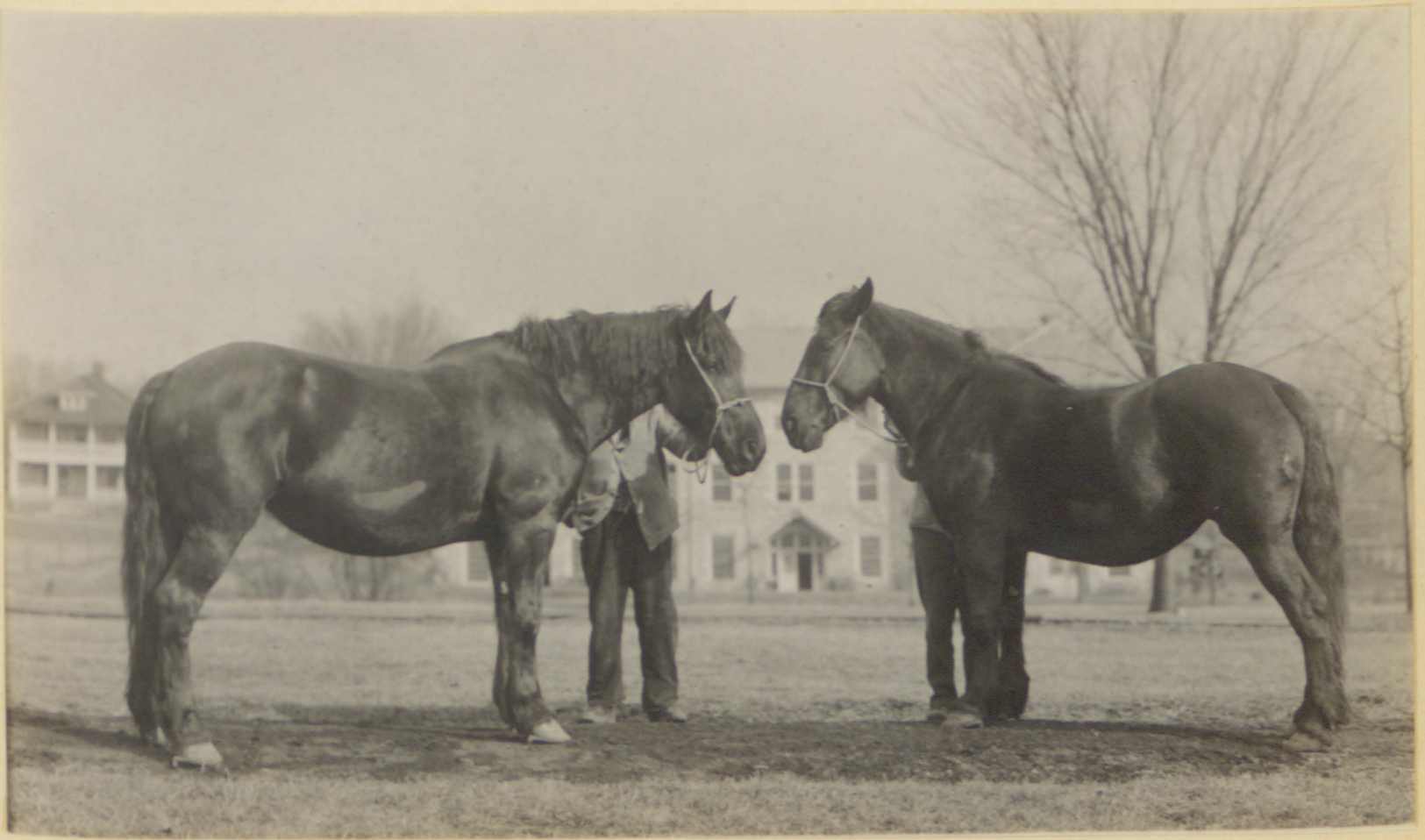


Tables XXVI and XXVIA present the data for Group B-c, Lots I and II, mature pregnant mares perfoming aversge winter farm work, Felibree, No. 1 and Forgette, No. 2.

Lot I received silage as a part ration. Lot II was fed no silage. Both lots were fed grain and timothy hay. These mares were in medium breeding condition when the test began. Forgette, No. 2, was nearly 100 pounds heavier. The initiel weight of mare No. I was 1533 pounds and the initial weight of mare No. 2 was 1630 pounds.

This team performed 443 hours of heavg labor and 44 hours of medium and light labor during the experiment.

Mare, No. I lost 43 pounds and mare No. 2 gained 7 pounds during the feoding period.

These mares produced foals in the spring of 1915 and suckled their foels until september 10, 1915.

Felibree, No. 1 was bred Jane 6, 1915 and Forgette, No. 2 was bred June 21,1915 .

Table XXVI shows that Lot II was fed an average of . 845 pounds of grain and 7.459 pounds of hay per daj more than Lot I. Lot I receired 13.452 pounds of silage per day.

From the table of digestible nutrients it will be noted that Lot II received 2.856 pounds of dry matter, .493 pounds of carbohydrates and .121 pounds of ash per day more than Lot I. Lot I was fed per day .067 pounds of protein and .084 pounds of fat more than Lot II.

Then reduced to a thousand pound basis Lot I con- 
sumed slightly less dry matter and ash per day than lot II. Lot I received per thousand pounds .105 pounds of protein, .105 pounds of protein, .271 pounds of carbohydrates and .059 pounds of fat more dally than Lot II.

There was practically no difference in the condition of flesh of these mares at the close of the trial. The hair and skin of Lot I indicated excellent thrift. 


\section{TABIE XXVII}

GROUP C

GRONING HORSES AND NULES

b - I Draft fogls

Lot I rilly foal, No. 17, Dam, Josephine,

Sire, Honorable

Lot II Filis foal, No. 18, Dam, Forgette, Sire, Honorable

FEED CONSUIED*ALD WEIGHMS*BY WEEKCY PERIODS

\section{IDOT I}

DAME $I_{\text {GRAIN }} 2_{\text {HAY }}$ 1915

Deo. 17

" $24 \quad 28$

" $31 \quad 56$ 1916

Jan. $7 \quad 42 \quad 35$

" $14 \quad 42$

" $21 \quad 42$

" $28 \quad 42$

$\mathrm{F} \otimes \mathrm{b}, 4 \quad 42$

" 1142

" 1856

n $25 \quad 56$

Hoh. 356

n 1063

Total $567 \quad 420$

Daily

Ave.
$6.75 \quad 5$

3.997
IOT II

SILAGE WEIGHT $I_{\text {GRAIN }} 2_{\text {HAY WEIGHT }}$

3773

$16 \quad 760$

780

770

770

800

800

820

840

820

845

870

863

$335.75 \quad{ }^{4} 90$

567

42

42

42

42

42

42

56

56

56

63

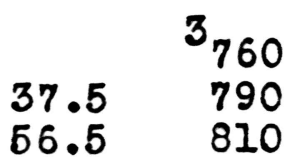

63

60

63

70

70

70

70

70

70

70

770

${ }^{4} 153$

\section{*Pounds}

1

corn, oats, bran, 2-2-1

2

Alfalla hay

3

Initial woight

4

Gain in woight 
TABIE XXVII

IOT I

DIGESTIBIE NUTRIENTS COMSUEED*

TOTAL FEED

CONSUNED

\section{D}

${ }_{\text {Hay }}^{1}$ Grain

S1lage

Total

Av. Daily

$$
\begin{aligned}
& 567.0 \\
& 420.0 \\
& 335.75
\end{aligned}
$$

517.506

391.301

120.665

1029.472

12.255
CARBO-

EYDRATES FAT ASH

$\begin{array}{llll}48.505 & 299.404 & 15.758 & 18.102\end{array}$

$\begin{array}{llll}44.650 & 166.459 & 1.693 & 38.338\end{array}$

$\begin{array}{llll}5.308 & 68.050 & 3.750 & 6.587\end{array}$

$\begin{array}{llll}98.463 & 533.913 & 21.201 & 63.027\end{array}$

1.172

6.355

.252

DIGESTIBLE NUTRIENTS CONSUNED *

PER 1,000 POUNDS IIVE WEIGHT

Total

Av. Daily
1273.311

15.158
121.789

1.450
$659.967 \quad 26.000$

7.857

.310

77.955

.928

\section{IOT II}

DIGESTIBLE INURIENTS CONSURED*

$2_{\text {Hag }}^{1}$ Grain

Total

Av. Da1Iy

\section{0}

770.0
517.506

717.386

1234.892

14.701
48.505

81.859

299.404 305.174

15.758

3.103

18.102

$\begin{array}{llll}130.364 & 604.578 & 18.861 & 88.388\end{array}$

1.552

7.197 .225

1.052

DIGESTIBLE NUTRIENTS CONSULED*

PER 1,000 POUNDS IIVE WEIGHT

Total

Av. Daily
1476.437

17.577
155.863

1.856
723.179

8.609
22.550105 .675

$.268 \quad 1.258$

1'corn, oats, bran, 2-2-1

2alfalfa

*Pounds 
GROUP C - b - 1

PHOTOGRAPHED AT THE BEGINNING OF EXPERIMENT, DECHMBER 18, 1915 IOT II DRAFT FOAL, NUNBER 18 IOT I DRAFT FOAL, NUUBBR I7 DAM, FORGETTE, SIRE, HONORABLE-DAM, JOSEPHINE, SIRE, HONORABLT

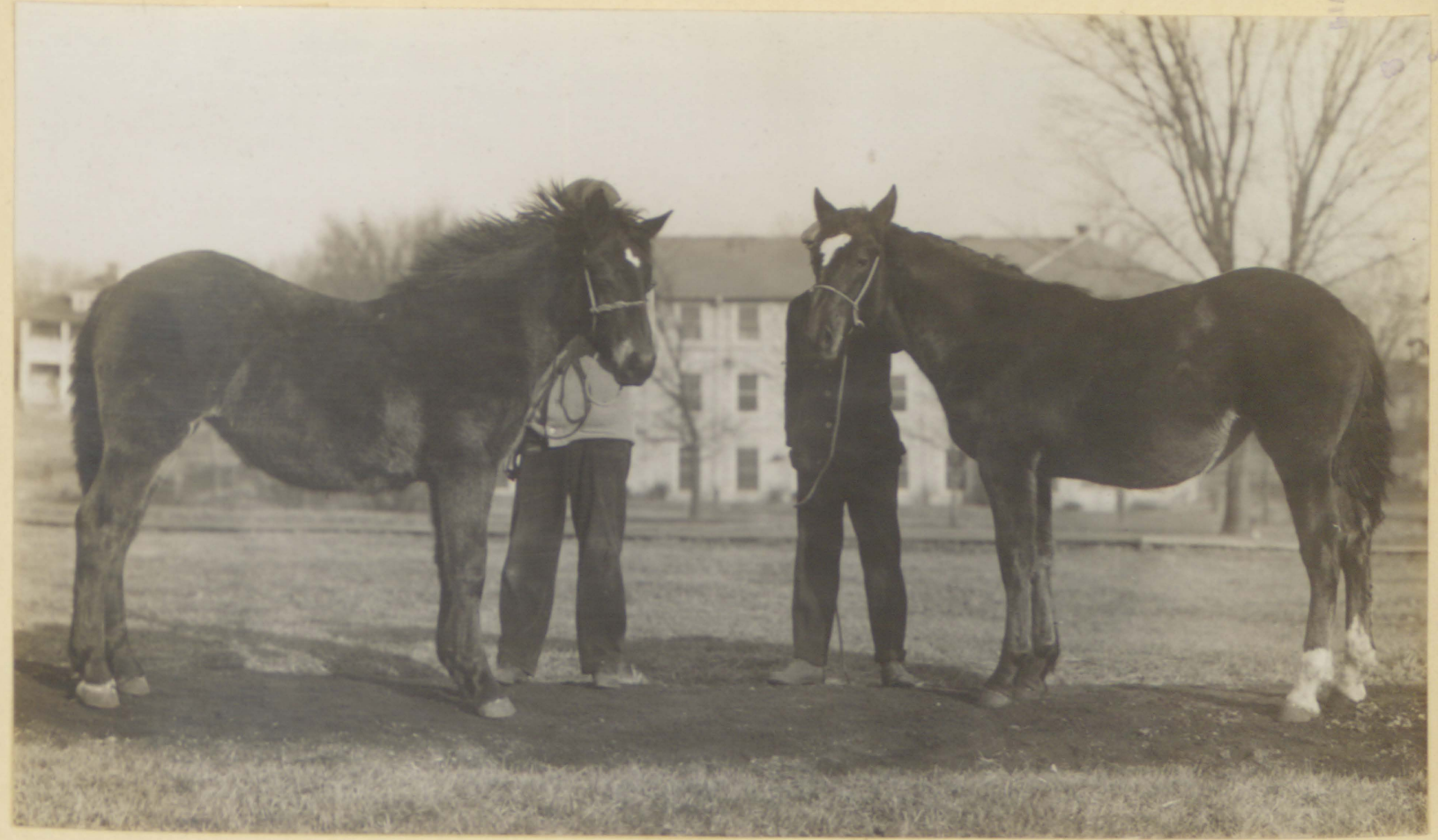

PHOTOGRAPHED AT THE CLOSE OF THE EXPERIMENT, MARCH 11, 1916

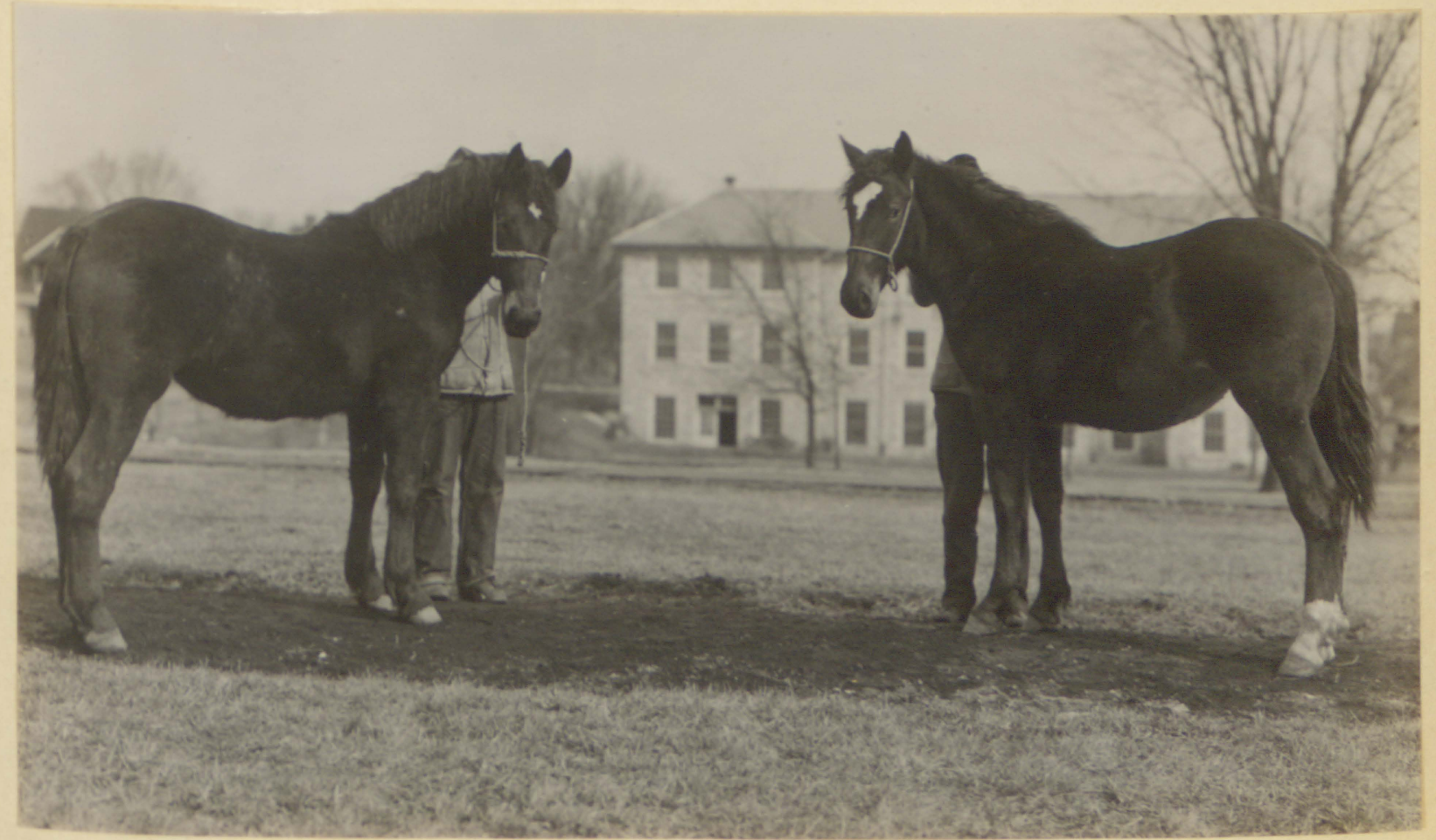


Tables XXVII and XXVIIApresent the date for Group $c-b-I$, Lots I and II, Percheron 11Ily foels, Numbers 17 and 18.

Lot I received silage as a part ration. Iot II was fed no sllage. Both lots were fed grain and alfalfa hay.

At the beginning of the test Iot I weighed 773 pounds and Lot II weighed 760 pounds. There was practically no difference in the condition of these colts when the test began. Lot I gained 90 pounds and Lot II gained 153 pounds during the test. Lot I was foaled April 6, 1915 and Lot II was foaled April 11, 1915. They suckled their dams until september 10, 1915.

Both lots consumed the same quantity of grain during the test. Lot II was fed an average of 4.167 pounds of hay more per day than Lot $I$. Lot I received an average of 3.997 pounds of silage per day.

The table of digestible nutrients shows that Lot II recoived an average of 2.446 pounds of dry matter, .38 pounds of protein, .842 pounds of carbohydrates and .302 pound 8 of ash daily in excess of that consumed by Lot I. Lot I receired da1ly .027 pounds of fat more than Lot II.

When reduced to a thousand pound basis, the table shows that Lot II consumed daily 2.429 pounds of dry matter. .406 pounds of protein, .752 pounds of carbohydrates, .33 pounds of ash more than Lot I. Lot I received slightly more fat than Lot II. 
Lot II was in slightlg better condition of flesh than Lot I at the close of the test. Lot I was in better thrift than Lot II as was indicated by her hair and skin. 


\section{TABIB XXVIII}

GROUP C

GROWING HORSES AND MULES

b - I Draft f081s

Iot I Plily foal, Ho. 19, Dam, Felibree, Sire, Honorable

Lot II Pilis foal, No. 20, Dam, Cervera, sire, Honorablo

FRED CONSUIOE

IOT I

\section{DATE $1_{\text {GRAIN }}$} 1915

$\begin{array}{ccc}\text { Doo. } 17 \\ n & 24 \\ n & 31\end{array}$

1916

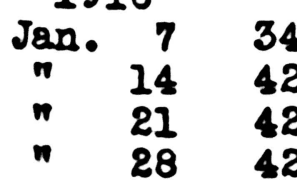

Fob. $4 \quad 42$

" $11 \quad 42$

" $18 \quad 56$

" $25 \quad 65$

Moh. $3 \quad 56$

63

28

33.5

$17.5 \quad 17.5$

35

17.5

17.5

17.5

17.5

17.5

21.0

19.0

19.5

21.0

35

21

21

23

28

28

34.5

35 .

35

29.5

$254 \quad 319.5$

Total

530

Da1ly

Aro.

IOT II

WEIGHT $I_{\text {GRAIN }} 2_{\text {HAY }}$ WEIGHT 36

710

$3_{590}$

$\begin{array}{llll}710 & 42 & 56 & 610\end{array}$

$\begin{array}{llll}690 & 34 & 56 & 630\end{array}$

$720 \quad 42 \quad 56 \quad 640$

$750 \quad 42 \quad 56 \quad 600$

$720 \quad 42 \quad 56 \quad 650$

$710 \quad 42 \quad 56 \quad 680$

$\begin{array}{llll}720 & 42 & 56 & 700\end{array}$

$710 \quad 56 \quad 55 \quad 700$

$710 \quad 56 \quad 56 \quad 720$

$710 \quad 56 \quad 56 \quad 730$

$\begin{array}{llll}705 & 63 & 56 & 733\end{array}$

${ }_{8}^{4} \quad 545 \quad 667.5 \quad{ }^{4} 143$

$6.488 \quad 7.946$

${ }^{1}$ Corn, oqts; bran, $2-2-1$

2

Alfalfa hay

3

Initial weight

4ain in woight

*Pounds 


\section{GROUP $C-b-1$}

PHOTOGRAPHED AT THE BEGINNING OF EXPERTMENT, DECEMMBER 18,1915 IOT II DRAFT FOAL, NUNBBER 20 IOT I DRAFT FOAI, NUNBBR 19 DAM, CERVERA, SIRE, HONORABIE-DAM, FEIIBREE, SIRE, HONORABIE

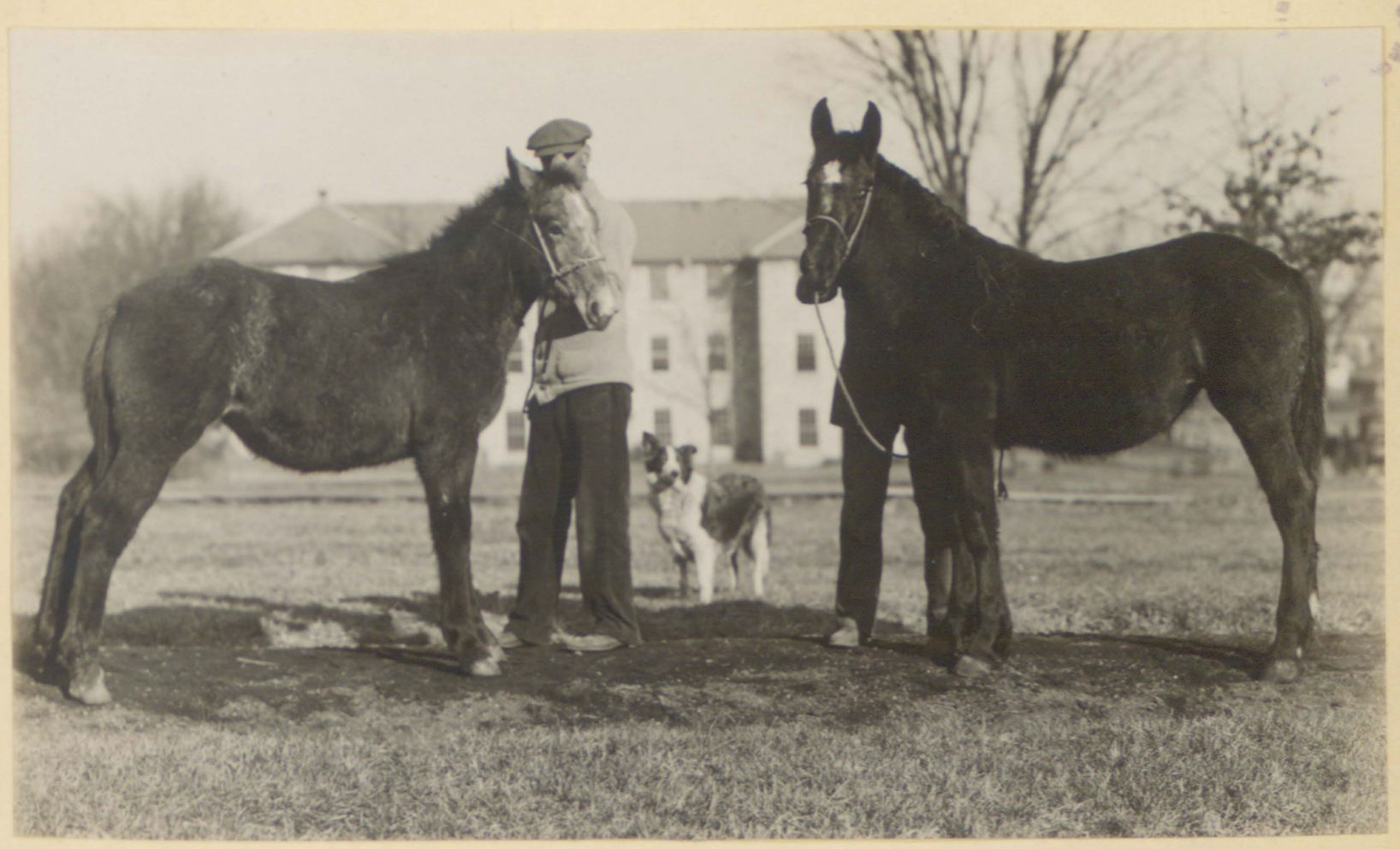

PHOTOGRAPHED AT THE CLOSE OF THE EXPRRIMENT, MARCH 11, 1916

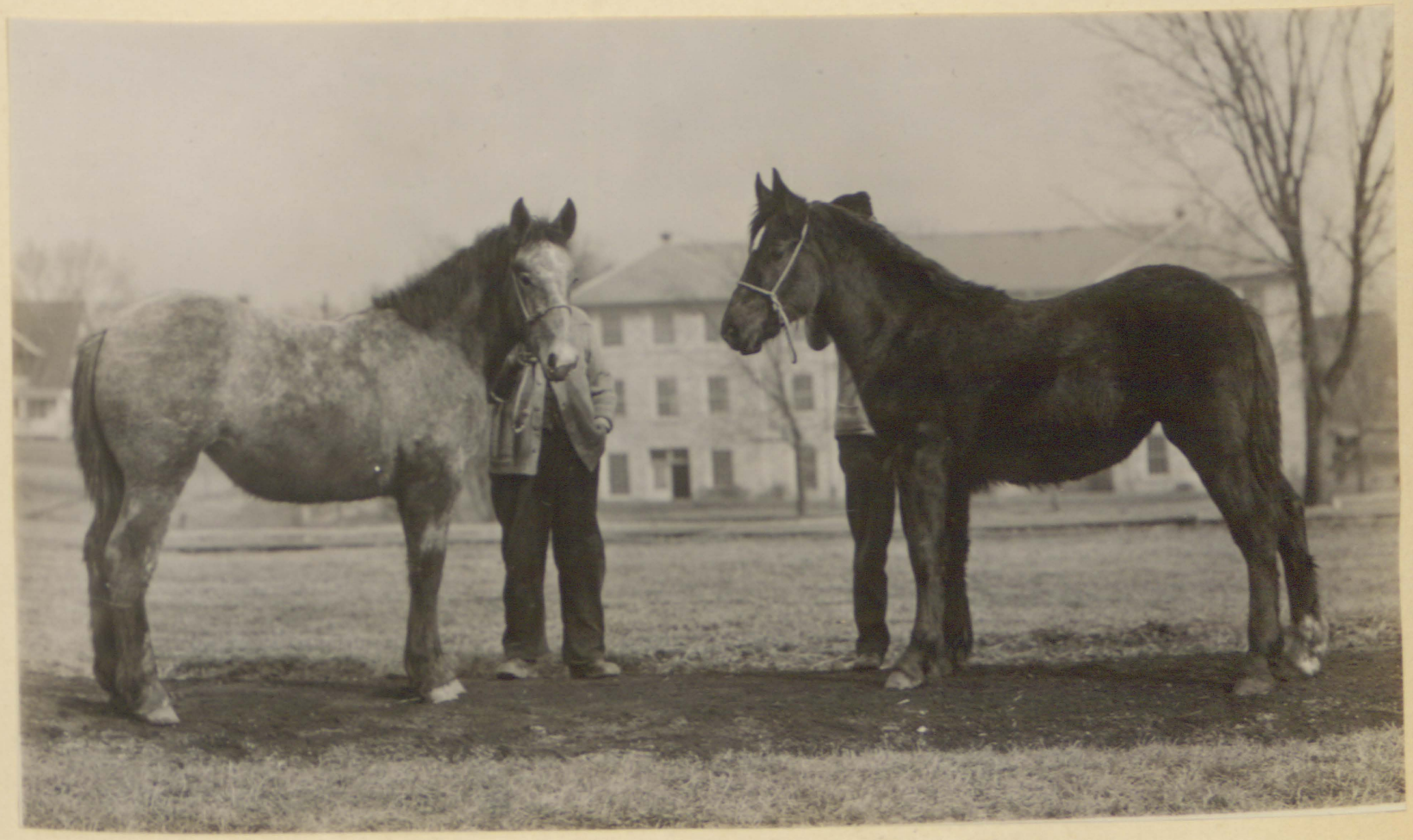


Table XXVIII presents the feed record and weights by weekly periods for Group $\mathrm{C}-b-I$, Lots I and II, Percheron f1lly foals, Numbers 19 and 20.

Lot I receired silage as part ration. Lot II was fed no silage.

Foal No. 19 was scouring at the beginning of the test. December 20, 1915 timothy replaced alfalfa in the ration and no sllage was fed. December 24, 1915, the foal, No. 19 was again put on the silage and alfalfa hay ration. January 23, 1916 she was scouring badly again and was put on a ration of grain, timothy hay and silage because the alfalfa had seemed to disagree with her.

The table of digestible nutrients and discussion are omitted for the reas on that the ration for Lot I varied so from time to time that the lote are not comparable. 


\section{TABIB LIX}

GROUP C

GRORING HORSES AND MULES

b - 2 Draft jearlings

Lot I Joscelin, No. 15, Registered Percheron Mare

Lot II Yearling gelding, Dam, Pinette, Sire Gardiner

FEED CONSUIED* AND WEIGHTS* BY WEEKLY PERIODS

\section{IOT I}

DATS 1915

De०. 17

ก 3170

1916

$\begin{array}{ll}\operatorname{Jan} & \\ n & 14 \\ n & 28\end{array}$

470

" 1170

" $18 \quad 70$

1 2570

Moh. 370

Total 840

Daily

ATo.

28

35

70

35

35

35

35

35

48

42

42
$10 \quad 5.667 \quad 13.604$

42

$476 \quad 1142.75$

56.

84

98

105

105

112

119

119

119

119

$50.75 \quad \begin{array}{r}3133 \\ 1140\end{array}$

1130

1130

1140

1130

1140

1160

1160

1150

1168

1200

1183

${ }^{4} 50$

840

$1_{\text {GRA }}$
70
70

$1_{\text {GRA }}$
70
70

56

IOT II

70

91

91

91

70

70

112

70

70

112

70

112

70

70

70

112

112

112

112

1218

10

14.5

${ }^{1}$ corn, oats, bran, $2-2-1$

2

Alfalfa haj

3

Initial weight

tgain in woight

*Pounds 
TABLB $\operatorname{DXIX}^{A}$

IOT I

DIGESMIBLS NUTRIZNTS CONSUIER*

TOTAL FEED Consumas

${ }^{1} \mathrm{Gra1n}_{\mathrm{Hag}}$

S11080

Total

Av. Daily

Total

Av. Daily
840.0

476.0

1142.75
DRY MATTER

766.680

443.475

407.288

1617.443

19.255
CARBO-

HYDRATES FAT ASH

$\begin{array}{lllll}71.908 & 443.455 & 23.346 & 26.894\end{array}$

$\begin{array}{llll}50.606 & 188.653 & 1.918 & 43.449\end{array}$

$\begin{array}{llll}18.067 & 231.601 & 12.765 & 22.421\end{array}$

$\begin{array}{llll}140.581 & 863.709 & 38.029 & 92.764\end{array}$

$\begin{array}{llll}1.674 & 10.282 & .453 & 1.104\end{array}$

DIGESTIBLE NUTRIENMS CONSUAED*

PER 1,000 POUNDS IIVE WEIGHT

$\begin{array}{rrrrr}1402.813 & 121.926 & 714.399 & 32.978 & 80.454 \\ 16.700 & 1.452 & 8.505 & .393 & .958\end{array}$

IOT II

DIGRSTIBLE NUTRIENTS CONSUMED*

$\operatorname{l}_{\text {Haj }}^{\text {Grain }}$

Total

4. Da1Iy

\section{0 \\ 1218.0}

766.680

1134.774

1901.464

22.636
$71.908 \quad 443.455$ $129.486 \quad 482.730$

$201.394 \quad 926.185$

2.398

11.026

$23.346 \quad 26.894$

4.897111 .179

28.243138 .073

$.336 \quad 1.644$

DIGESTIBLE NUTRIBNTS CONSU:TED*

PER 1,000 POUNDS LIVB WEIGET
Total

Ar. Daily
1649.136

19.632

${ }^{1}$ corn, oats, bran, $2-2-1$

2

Alfalfa haj

*Pound 8 


\section{GROUP B - b - 2}

PHOTOGRAPHED AT THE BEGINIING OF EXPERTMENT, DECEMMBER 18, 1915 IOT I JOSCELIN, NUMBER 15 IOT II YEARIING GEIDING, NUMBER 16 DAM, PINETITE, SIRE, GARDINER

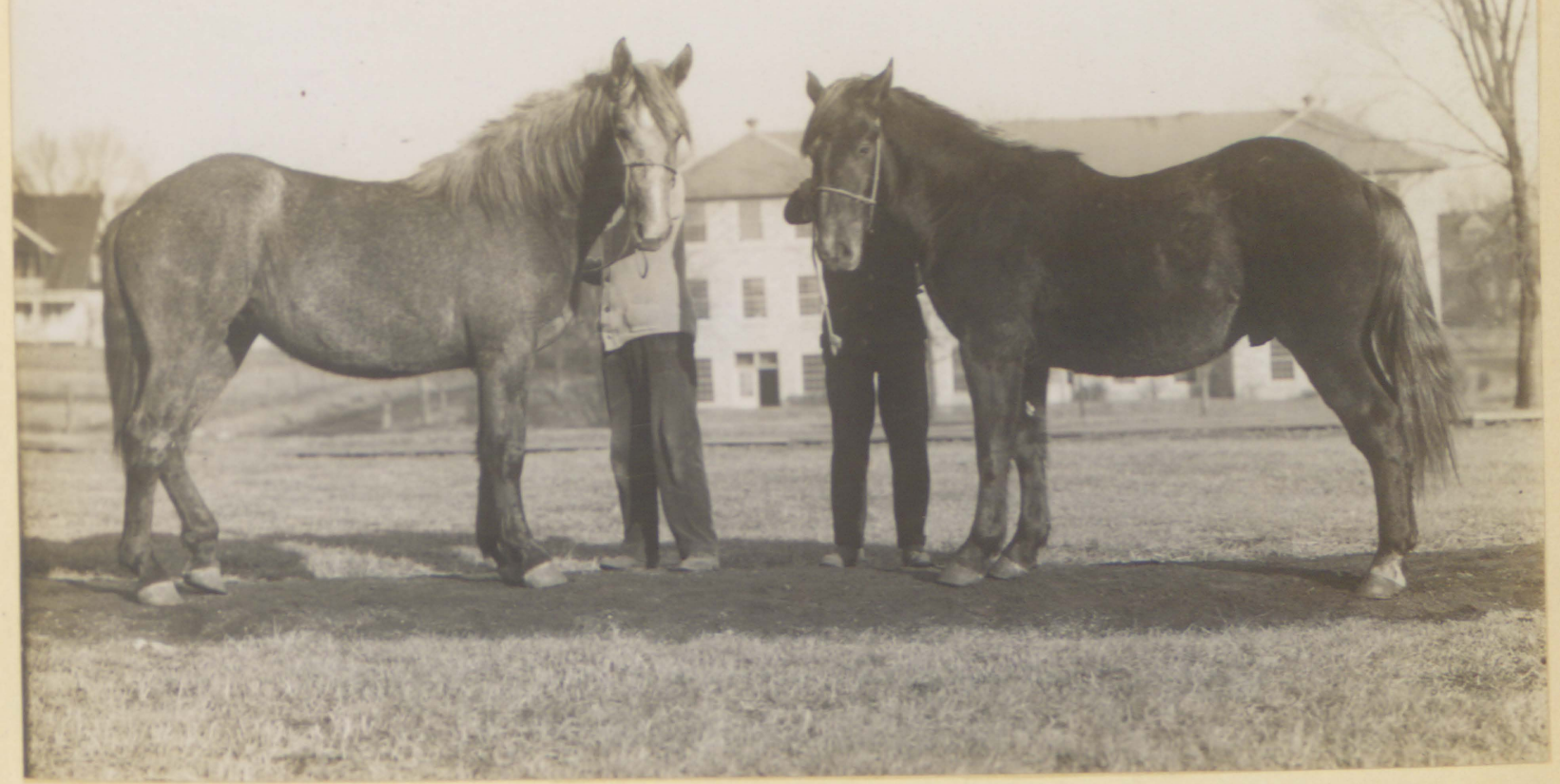

PHOTOGRAPHED AT THE CLOSE OF THE EXPERIMENT, MARCH 11,1916 LOT II YEARLING GELDING, NO.16 LOT I JOSCELIN, NUMBER I5

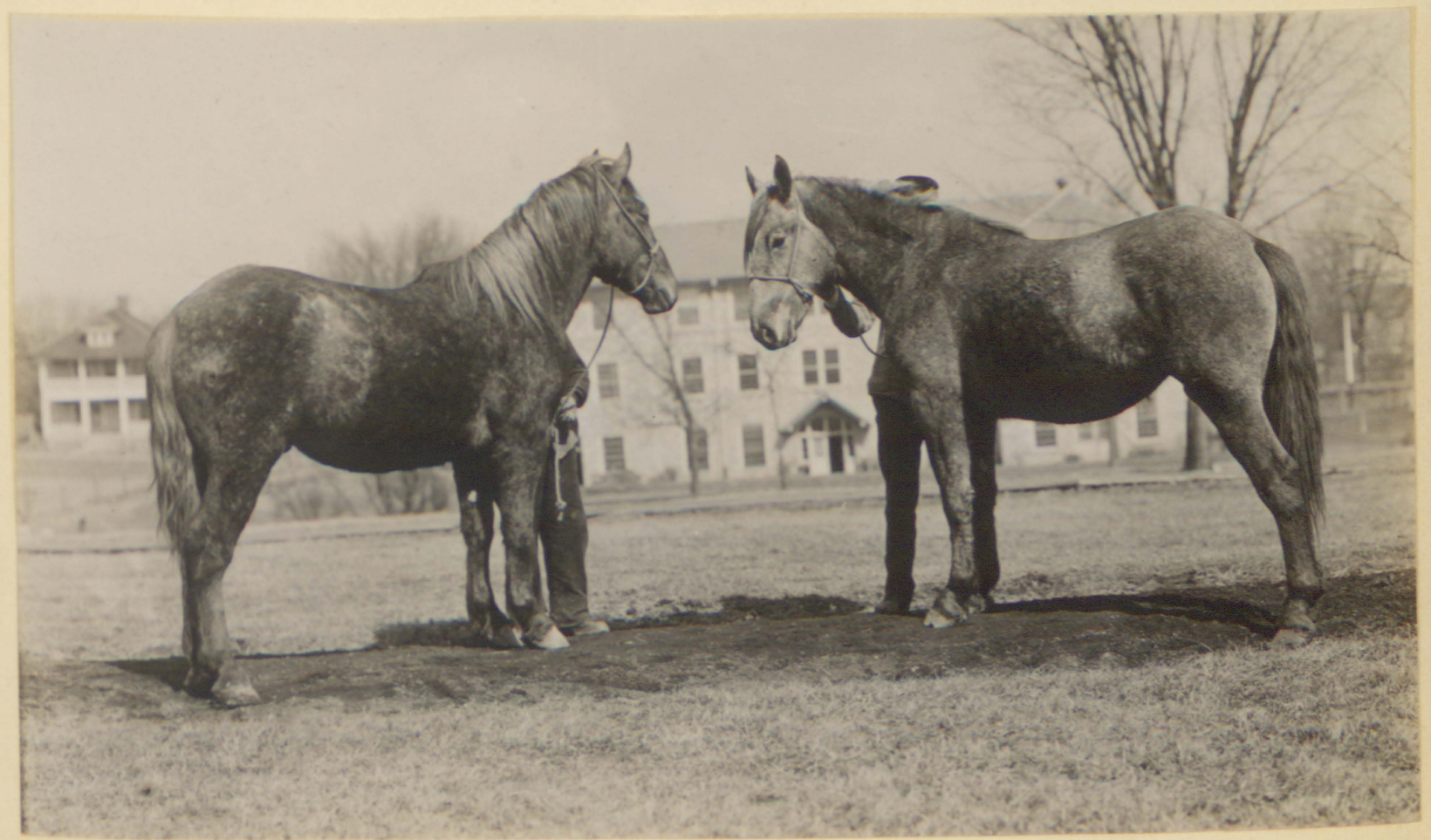

GROWING DRAFT YEARLINGS

LOT II YRARLING GELDING NO. 16 LOT I JOSCBLIN, NUNBER 15 NO SILAGE 
Tables XXIX and XXIX present the data for Group B-b-2, Lots I and II, dreft jearlings, numbers, 15 and 16. Lot I received silage as a part ration. Lot II was fed no silage. Both lots were fed grain and alfalfa hay. These draft yearlings were in medium growing condition at the beginning of the test. The initial weight of Lot I was 1133 pounds and the initial weight of Iot II was 1140. Iot I gained 50 pounds and Lot II gained 92 pounds during the test.

Both lots consumed the same quantity of grain. Iot I was fed 8.833 pounds of hay daily less than Lot I. Lot I consumed an arerage of 13.604 pounds of silage daily.

Lot I received dailg 3.381 pounds of dry matter, .724 pounds of protein, .744 pounds of carbohydrates and .54 pounds of ash less than Lot II. Iot I was fed .117 pounds of lat per day more than Lot II.

These facts hold true when reduced to a thousand pound basis for the reas on that the lots did not vary greatly In weights during the test.

At the close of the test lot I was in better condition and her hair and skin indicated better thrift than Lot II. 


\section{TABIR $X X X$}

GROUP C

GROWING HORSES AND WUIES

- - I Saddle Forls

Iot I stud foal, No. 2I, Dam, Ruth MCDonald, Sire, sstral King

Lot II Stud foal, No. 22, Dam, Iona Rex, Sire, Kentuckj's Best

WEED CONSUCED*AND WEIGHMS*BY WEEKIY PERIODS

\section{IOT I}

\section{DA'TB
1915}

De0. 17

n $24 \quad 28$

$\begin{array}{lll}1 & 31 & 28\end{array}$

1916

Jan. 728

$\begin{array}{lll}\text { " } & 14 & 28\end{array}$

" 2128

" $28 \quad 28$

Fob. 428

n 1128

" 1836

". 2542

Moh. 342

n $10 \quad 49$

Total 393

Da1ly

Avo. $2_{\text {HAY SIIAGE }}$

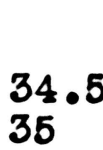

31

27.5

35

35

35

35

35

35

35

35

35

31

28

28

28

28

28

35

42

42

42

42

$415.5 \quad 381.5$

4.542

\section{WEIGHT \\ $1_{\text {GRAIN }}$}

$3_{583}$

591

610

580

560

560

570

620

640

605

620

640

643

${ }^{4} 60$
$2_{\mathrm{HAY}}$

WEIGHT

$3_{440}$

$\begin{array}{lll}33 & 32.75 & 470\end{array}$

$28 \quad 35$

490

480

485

480

500

510

570

500

510

525

518

$49 \quad 42$

478

${ }^{1}$ corn, oats, bran, $2-2-1$

${ }^{2}$ Alfalfa hag

3 Initial woight

4 Gain in woight

*Pound 8 


\section{TABIE $X X x^{A}$ \\ IOT I}

DIGESTIBIE NUTRIENTS CONSUMED *

TOMAL FEND CONSUEDD

$2_{\text {Grain }}$
$2_{\text {Haj }}$
S1lago

Total

Av. Daily

Total

AT. Daily

$$
\begin{aligned}
& 393.0 \\
& 415.5 \\
& 381.5
\end{aligned}
$$

DRY LIATMER PROTEIN

358.693

387.109

135.970

881.772

10.497

33.613
44.172
6.032

83.817

.999
CARBO-

HYDRATES FAT ASH

$207.538 \quad 10.921 \quad 12.536$

$\begin{array}{lll}164.675 & 1.674 & 37.927\end{array}$

$\begin{array}{lll}77.319 & 4.216 & 7.485\end{array}$

$\begin{array}{llll}449.532 & 16.811 & 57.948\end{array}$

$5.352 \quad .200 \quad .690$
DIGESTIBLE NUTRIENTS CONS UAED*

PER 1,000 POUNDS IITS WEIGHS

DIGESTIBIE MUTRIEUTS CONSUIED*

$I_{\text {Gay }}^{1}$

Total

Av. Daily
IOT II

1464.738

17.437

445.75
355.956
415.292

33.364 47.388

771.248

9.182 .961
139.231

1.658
746.731

8.888
27.925

.332
96.259

1.146
DIGESTIBLE NUTRIENTS CONSUEED* PER 1,000 POUNDS LIVE WEIGHT
Total

Av. Daily
1548.683

18.437

162.153

776.225

1.930

$205.937 \quad 10.839 \quad 12.452$ $\begin{array}{lr}180.627 \quad 1.796 & 40.688\end{array}$ $80.752 \quad 386.564 \quad 12.635 \quad 53.140$

4.602 .150 .633

${ }^{1}$ Corn, osts, bran, $2-2-1$

¿alfalfa

*Pound 8 
GROUP $C-0-1$

PHOTOGRAPHKD AT THE BEGINNING OF EXXPERTMENT, DECEMBBER 18, 1915 IOT II SADDLE FOAL, NUMBER 22 -IOT I SADDIE FOAI, NUMBER $2 I$ DAM, IONA REX, SIRE, KRITUUCKY'S-DAM, RUTH YODONAID, SIRE, ASTRAI BEST KING

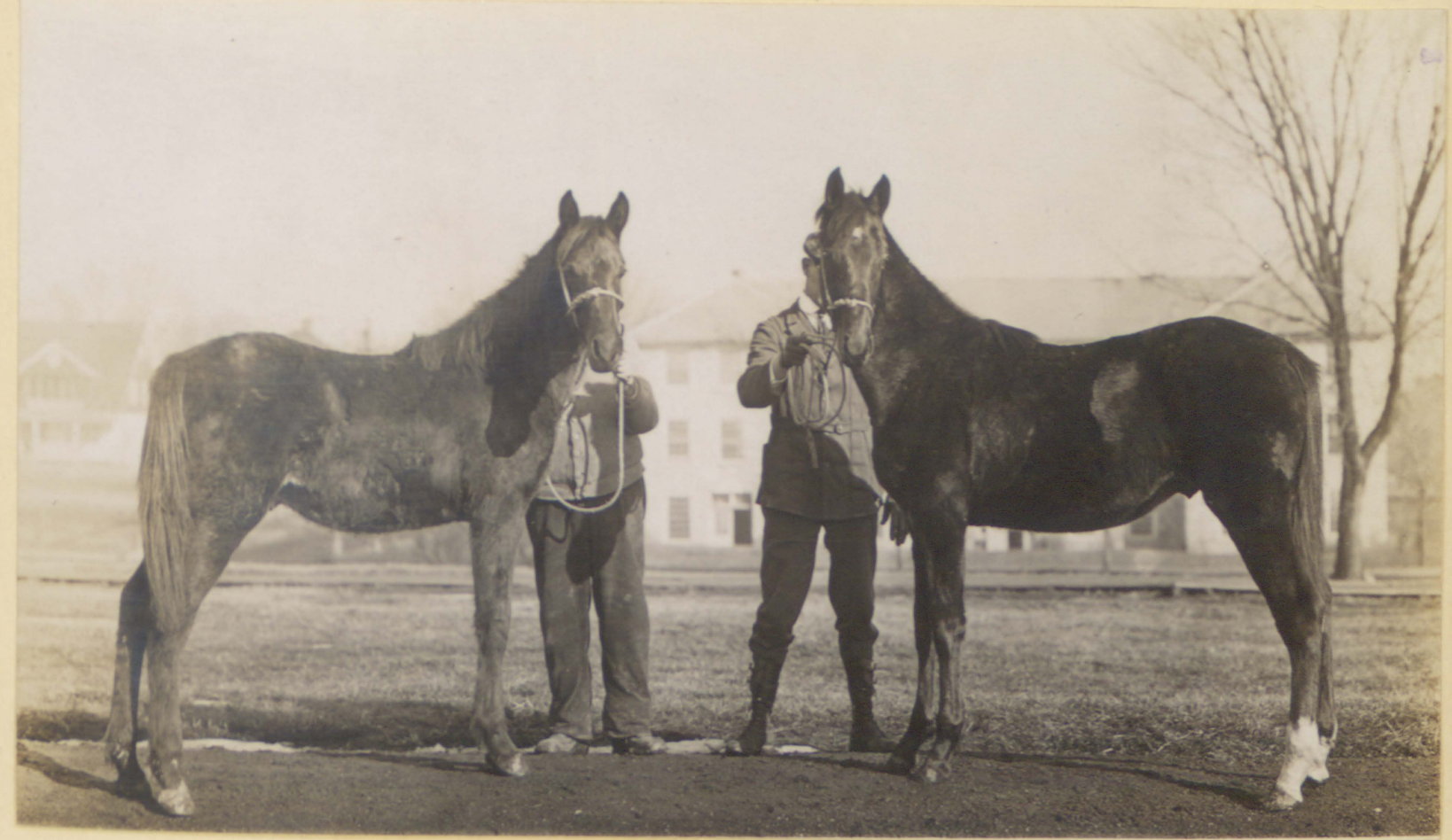

PHOTOGRAPHED AT THE CLOSE OF THE EXPFRIMGNT, MARCH 11, 1916

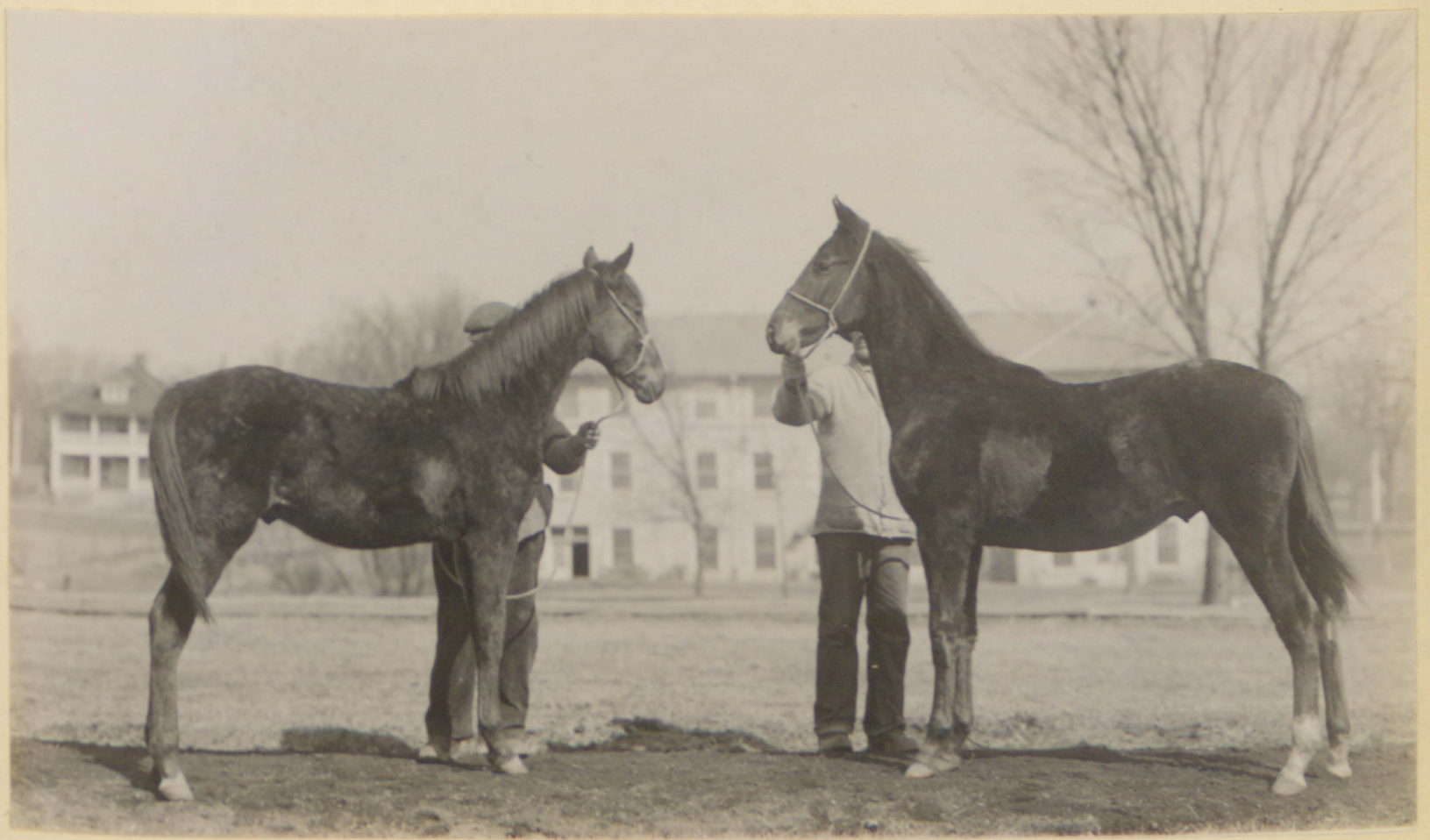


Tables $X X X$ and $X X X^{A}$ present the data for Group $C-C$, Lots I and II, saddle foals, numbers, 21 and 22.

Lot I received silage as a part ration. Lot II was fed no 8llage. Both lots were fed grain and alfalfa hag.

This pair of foals wes in medium growing condition when the test began. The initial weight of Iot I was 583 pounds and the inftial weight of Lot II was 440 pounds. Iot I gained 60 pounds and Lot II 78 pounds during the feeding period.

Lot I was fooled March 27, 1915 and Lot II was foaled April 6, 1916. Thej suckled their dams until september 10, 1915. Both lots were led practically the same quantity of grain. Lot I consumed daily .36I pounds of hay less than Iot II. An average of 4.542 pounds of silage was consumed daily by Lot I.

Table $X^{A}$ shows that Lot I consumed slightly more digestible nutrients than Lot II during the test.

When reduced to a thousand pound basis Lot II received daily an average of 1 pound of dry matter,.272 pounds of protein, .353 pounds of carbohydrates and .125 pounds of ash more than Lot I. Lot I received slightly more fat.

Lot I was in better condition of flesh than Iot II at the close of the experiment. The condition of the coat of hair of Lot I indicated that this lot was in better thrift than Lot II. 


\section{TABIE XXXI}

GROUP C

GROMING HORSES AND RULES

- - 2 Saddle bred yearlings

Lot I Sport, No. 13, Sadale bred geleing

Lot II Ruth Squirrel, No. 14, Registered sadale filly

FEED CONSUIED*AND WEIGHMS* BY WEAKLY PERIODS

IOM I

DATE $I_{\text {GRAIN }} 2_{\text {HAY }}$ 1915

$\operatorname{Dec}_{n} \frac{17}{24}$

n 21

1916

Jan. 728

" $14 \quad 28$

$\begin{array}{lll}n & 21 & 28\end{array}$

n $28 \quad 28$

Feb. $4 \quad 28$

n 1128

n 1836

n $25 \quad 42$

Moh. $\begin{array}{rr}3 & 42 \\ 10 & 48\end{array}$

392

28

35

51

49.5

35

35

35

35

35

35

35

35

35

33.5

56

56

84

84

84

89.75

89.25

87.75

87.75

86.75

$411.5 \quad 906.75$

$4.9 \quad 10.795$

Dails
Ave.

$$
4.667
$$

\section{IOT II}

$2_{\text {HAY WEIGHT }}$

3

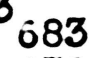

070

660

$I_{\text {GRAIN }}$

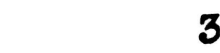

3790

28

56

785

28

91

720

665

28

91

770

665

680

28

91

770

28

91

780

700

28

112

112

$700 \quad \approx 8 \quad 112$

$690 \quad 36 \quad 112$

$70542 \quad 112$

710

42

112

702

48

108.5

770

780

790

800

810

815

813

$\begin{array}{llll}4_{19} & 392 & 1202.5 & { }^{4} 23\end{array}$

${ }^{1}$ Corn, oats, bran, $2-2-1$

¿nlfalfa hag

3 Initial woight

4

Gain in woight

* Pounde

$4.667 \quad 14.315$ 


\begin{tabular}{|c|c|c|c|c|c|}
\hline \multicolumn{6}{|c|}{ DIGESTIBLE NUTRIENTS CONSURED* } \\
\hline $\begin{array}{l}\text { TOTAL FERD } \\
\text { CONSUENED }\end{array}$ & DRY WATTER & PROTEIN & $\begin{array}{l}\text { CARBO- } \\
\text { HYDRATES }\end{array}$ & TAT & ASH \\
\hline \multirow[t]{3}{*}{$\begin{array}{l}392.0 \\
411.5 \\
906.75\end{array}$} & $\begin{array}{l}357.781 \\
383.382 \\
323.175\end{array}$ & $\begin{array}{l}33.528 \\
43.747 \\
14.336\end{array}$ & $\begin{array}{l}207.009 \\
163.090 \\
183.771\end{array}$ & $\begin{array}{r}10.893 \\
1.658 \\
10.128\end{array}$ & $\begin{array}{l}12.505 \\
37.562 \\
17.790\end{array}$ \\
\hline & 1064.338 & 91.611 & 553.870 & 22.679 & 67.857 \\
\hline & 12.671 & 1.090 & 6.594 & .270 & .808 \\
\hline
\end{tabular}

DIGESTIBLE NUMRIENTS CONSURAR*

PER 1,000 POUNDS IIVE WEIGHT

Total

A7. Da1ly
1551.513

18.470
$133.544 \quad 807.392$

1.590

9.612

33.060

98.917 $.394 \quad 1.178$

IOT II

DIGBSTIBLE WUTRIENTS CONSUNAD*

$\log _{\text {Haj }}^{\text {Zara }}$

Total

Ar. Da1ly

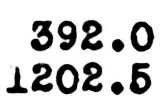

357.781 1120.343

1478.124

17.597 $\begin{array}{ll}33.528 & 207.009\end{array}$ $127.838 \quad 476.587$

$10.893 \quad 12.505$ 4.846109 .764

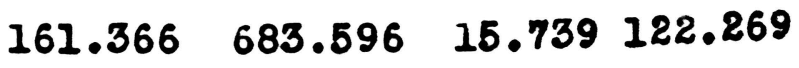

1.921

8.138 1.456

DIGESTIBLE NUTRIENTS CONSURED* PER 1,000 POUNDS II VE WEIGET

Total

Ar. Daily
1885.362

22.445
205.824

2.450
$871.926 \quad 20.075155 .955$

10.380 $.239 \quad 1.857$

${ }^{2}$ Corn, oate, bran, $2-2-1$

2 Alfalfa hay

*Pound 8 


\section{GROUP $\mathrm{C}-0-2$}

PHOTOGRAPHED AT THE BEGINIIIIG OF EXPERTMENIT, DECEMBER 18, 1915 IOT II RUTH SQUIRREL, NUMBBER 14 IOT I SPORT, NUUBBER 13

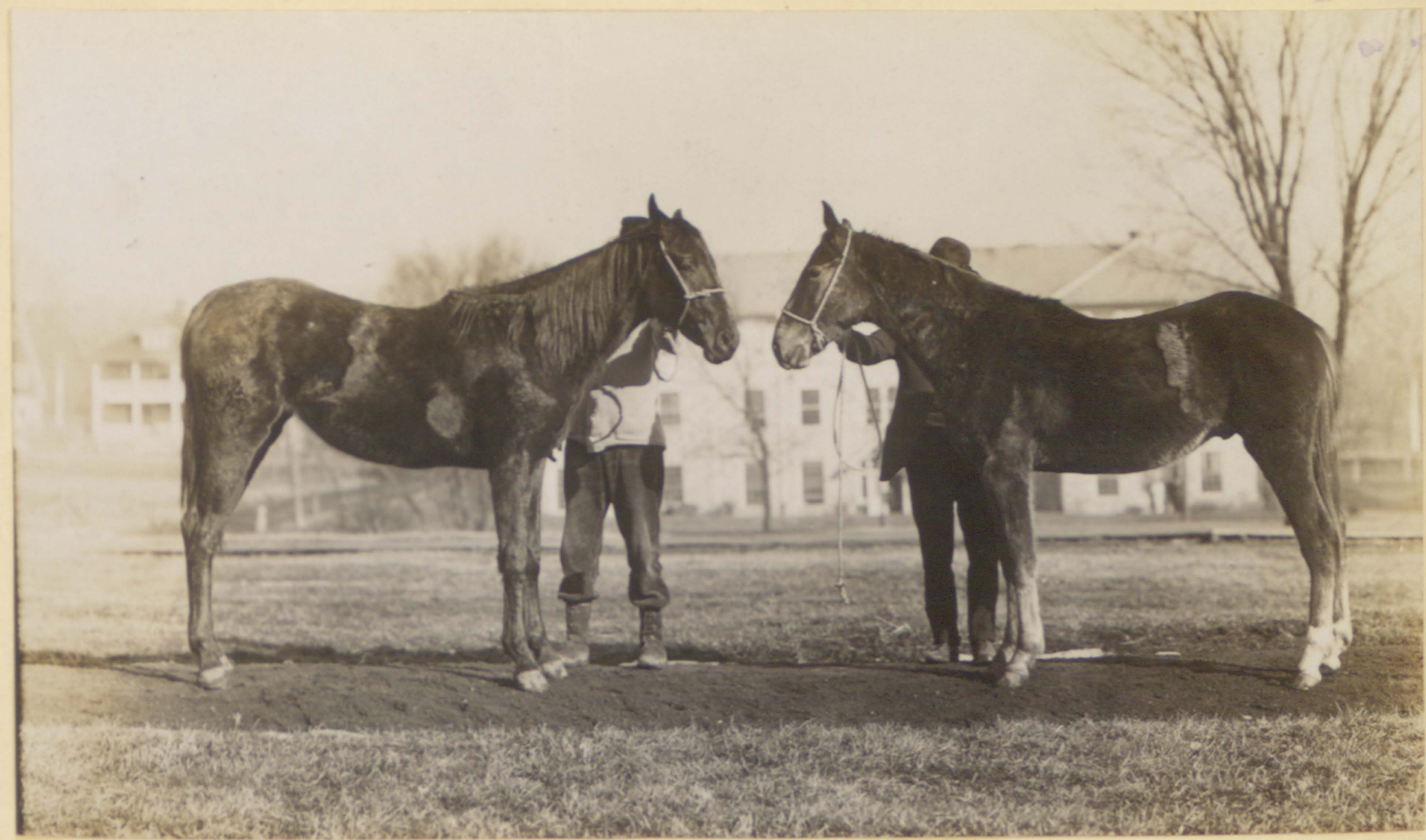

PHOTOGRAPHED AT THE CLOSE OF THE EXPERIMENT, MARCH 11, 1916

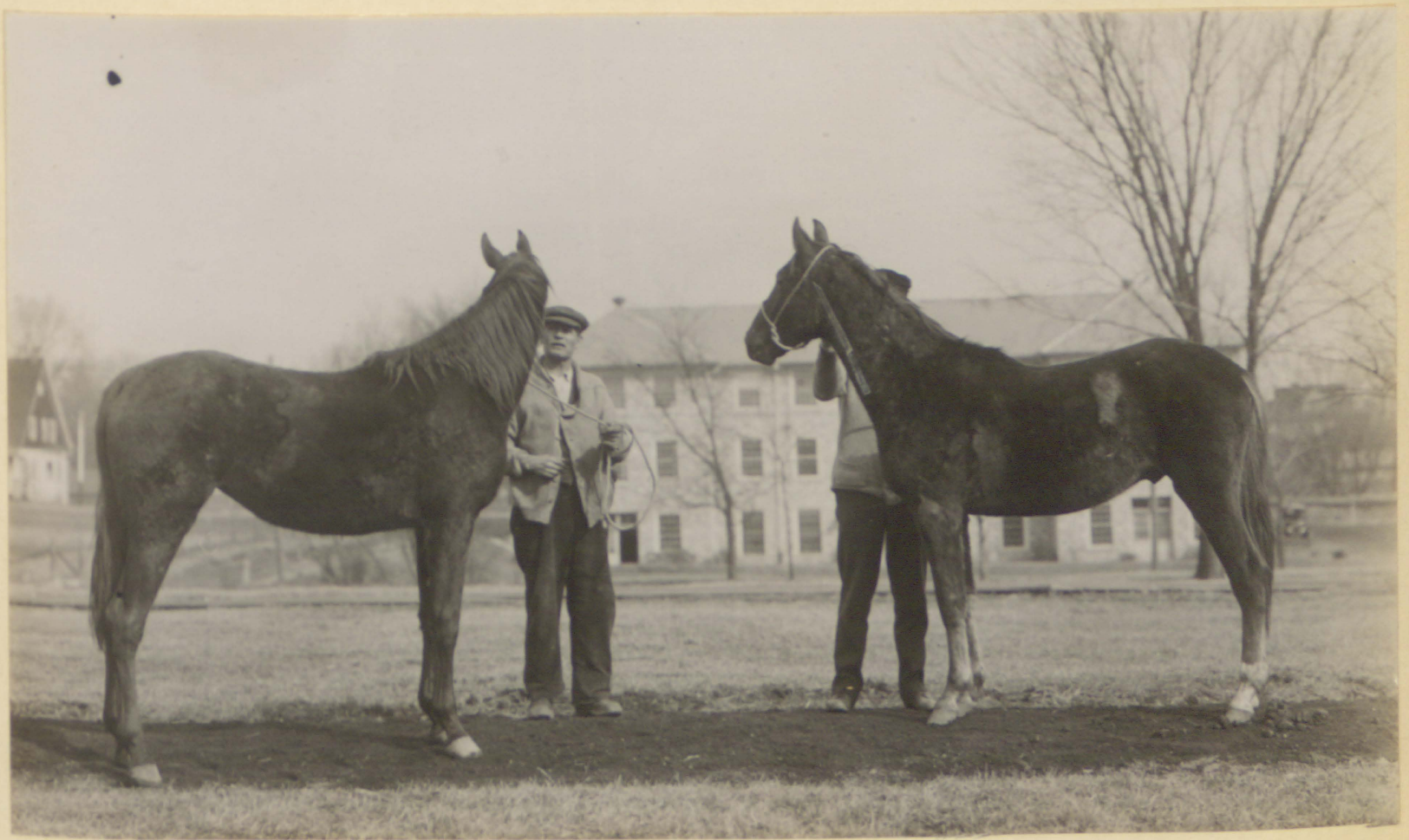

GROWING SADDLE YBARLINGS 
Tables XVXI and XXXYA present the data for Group C-o, Iots I and II, saddle bred yearlings, Sport, No. 13 and Ruth Squirrel, No. 14.

Lot I recoired silage as a part ration. Lot II received no silage. Both lots were fed grain and alfalfa hay.

At the begirning of the experiment these yearlings were in medium growing condition. They were not fat. Iot I weighed 683 pourds when the test began and Lot II 795 pounds. Lot I gaired 19 pounds and Lot II gaired 23 pounds during the feedirg period.

Both lots received the same amount of grain. Iot I was fed 9.415 pounds of hay per day less than Lot II. A daily averge of 10.795 pounds of silage was consumed by Lot $I$. Table XXXI shows that Lot II receired daily 4.926 pounds of dry matter, .831 pounds of protein, 1.544 pounds of carbohydrates and .648 pounds of ash more than Lot I. Iot I received per day .083 pounds of fat more than Lot II.

The table of digestible nutrients consumed per thousand pounds live weight shows that Lot II consumed 3.975 pounds of drg matter, .860 pounds of protein, .768 pounds of carbohydrates and .679 pounds of ash more than Lot I. Lot I received .155 pounds of fat more than Lot II.

Lot II was in slightly better condition than Lot I at the close of the test. 


\section{TABIE DOXII}

GROUP D

IMOMATURE HORSES AND MULES PERFORMING AVERAGB WINTER FARI WORK

c. Pregnant mares

Lot I Mena, 110. 3, Registered Percheron mare

Lot II Josephe, No. 4, Registered Percheron mare

IBEED CONSUMED, WORK HOURS AND WEIGHMS* BY WEEKCIY PERIODS

$$
\text { LOT I LOT II }
$$

DATS ${ }^{2}$ GRAIN ${ }^{2}$ HAY SILAGB WBIGHT ${ }^{2}$ GRAIN $2_{\text {HAY }}$ WEIGHT ${ }^{5}$ WORK

1915

Dec.17

(1) 2

" $31 \quad 100 \quad 65$

1916

Jan. $7 \quad 100 \quad 70$

" is $100 \quad 61$

" $21 \quad 100 \quad 62$

" $28 \quad 10068$

Fob. $4 \quad 100$

" 11100

- 18100

- 25100

Moh. $3 \quad 100$

70

67.5

52.5

50

46.5

53.0

\begin{tabular}{lrll}
\multicolumn{5}{c}{$\mathbf{3}_{1517}$} & & \\
27.25 & 1500 & 100 & 105 \\
$\mathbf{5 4}$ & 1480 & 100 & 105 \\
56 & 1500 & 100 & 105 \\
56 & 1470 & 100 & 105 \\
70 & 1500 & 100 & 105 \\
70 & 1480 & 100 & 126 \\
70 & 1520 & 100 & 126 \\
$\mathbf{8 4}$ & 1540 & 100 & 126 \\
$\mathbf{8 8 . 5}$ & 1510 & 111 & 134 \\
91.0 & 1520 & 120 & 140 \\
76.0 & 1540 & 120 & 138 \\
$\mathbf{8 8 . 5}$ & 1557 & 120 & 140
\end{tabular}

Total 1200

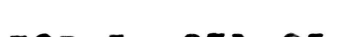

$4_{40}$

$1271 \quad 1455$

$\begin{array}{rrr} & A & B \\ 1567 & & \\ 1560 & 40 & 10 \\ 1560 & 29 & \end{array}$

$1550 \quad 35$

$1500 \quad 37 \quad 5$

$1550 \quad 224$

$1510 \quad 35$

$\begin{array}{lll}1550 & 26 & 9\end{array}$

$\begin{array}{lll}1565 & 27 & 7\end{array}$

153031

155046

$15503_{6}$

1570

$43 \quad 358 \quad 35$

Deily

Are.

$14.286 \quad 8.637 \quad 9.896$

$15.131 \quad 17.321$

*Pounds

${ }^{1}$ corn, oats, bran, $2-2-1$

EImothy hay

3

In1tial woight

Gain in woight

$\sigma_{\Lambda}$ - hearg work, B - modium and light work

${ }^{6}$ stopped working team until ofter Mena foaled 


\section{TABIR XXXII \\ IOT I}

DIGESTIBIE NUTRIENTS CONSUNIED*

TOTAL PEED CONSURED

$2_{\text {Hay }}^{1}$

Slloge

Total

Dailg Ar.

$$
\begin{gathered}
1200.0 \\
725.5 \\
831.25
\end{gathered}
$$

$$
\begin{aligned}
& \text { DRY NATTER } \\
& 1094.977 \\
& 677.305 \\
& 296.266
\end{aligned}
$$$$
2068.548
$$$$
24.626
$$

PROTEIN ETD

CARBO -

HYDRATES PAT ASH $\begin{array}{llll}102.726 & 633.507 & 33.140 & 38.420\end{array}$ $\begin{array}{llll}7.161 & 269.690 & 0.805 & 34.897\end{array}$ $\begin{array}{llll}13.142 & 168.469 & 9.285 & 16.309\end{array}$ ¿23.089 $1071.666 \quad 49.230 \quad 89.626$ $1.465 \quad 12.758$ $.586 \quad 1.067$ PER 1,000 POUNDS IIVE WEIGHT

Total

Dailg Ar.

$$
1369.899
$$

16.308
81.476

.970
$709.712 \quad 32.603$

8.449

59.355 .707

\section{LOT II}

DIGESTIBLE NUTRIEITS CONSURED*

$\log _{\text {Haj }}^{1}$

Total

Daily AT.

\section{0 1455.0}

$1159 \cdot 747$ 1358.344 29.976 $2518.091 \quad 123.128 \quad 1174.374 \quad 48.980102 .621$

108.767 14.36

633.507 540.867 35.332 40.635 61.986 29.976 .583 1.222

DIGESTIBLE NUTRIRNTS CONSURAB*

PER 1,000 POUNDS IITS WEIGEM

Totel

Daily Av.
1626.674

19.365
79.540

758.640 .947 9.031

$31.641 \quad 66.293$ .377 .789

${ }^{1}$ Corn, oats, bran, $2-2-1$

2

Timothy

* Pounds 


\section{GROUP C - 0}

PHOTOGRAPHED AT THE BEGINIING OF EXPERTIREINT, DECHIBER 18, 1915 IOT I MENA, NUMBER 3

IOT II JOSHPHE, NUMBER 4

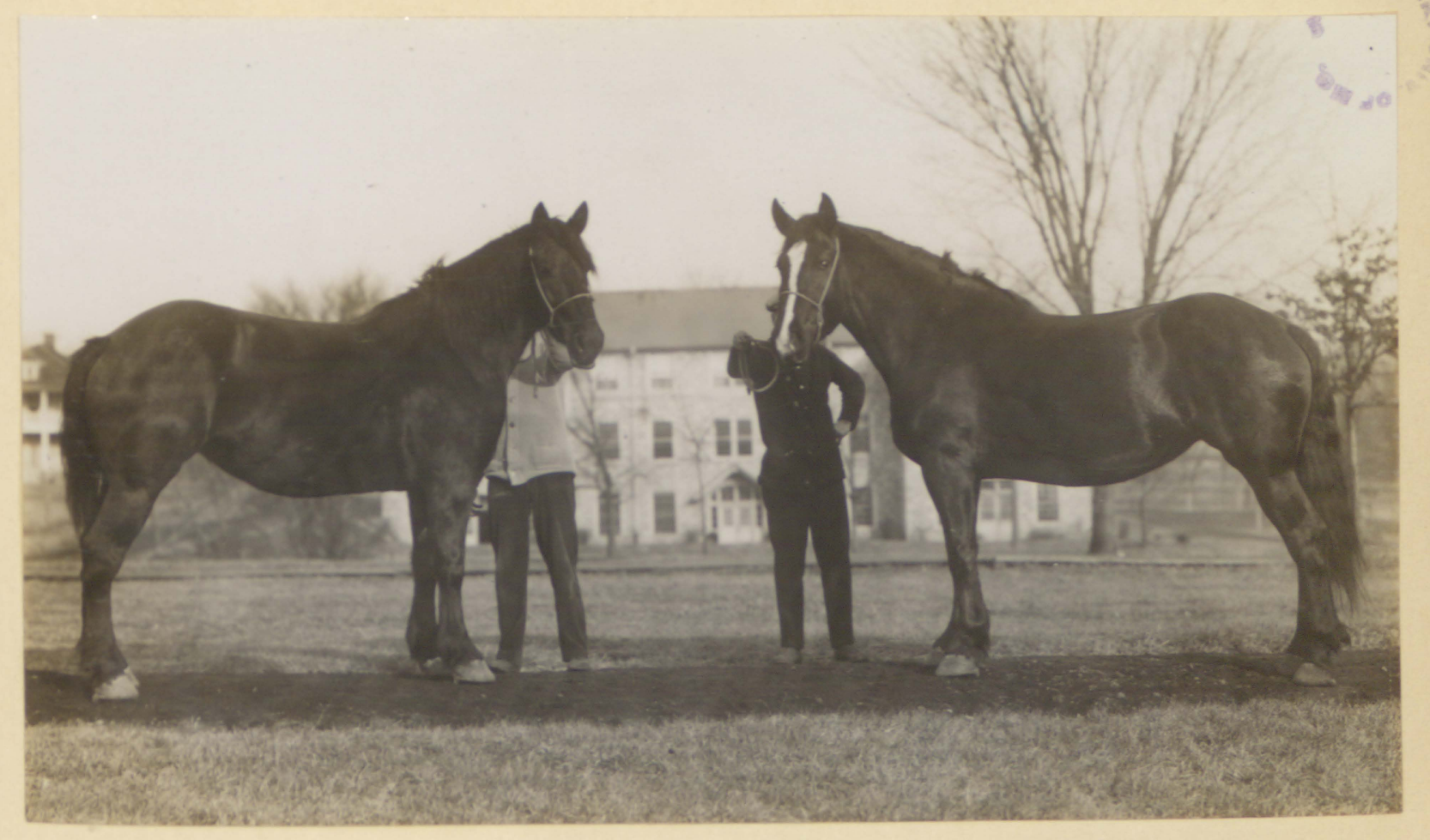

PHOTOGRAPHED AT THE CLOSE OF THR EXPER IMENT, MARCH 11, 1916 IOT II JOSEPHE, NUMBER 4 LOT I MENA, NUMBER 3

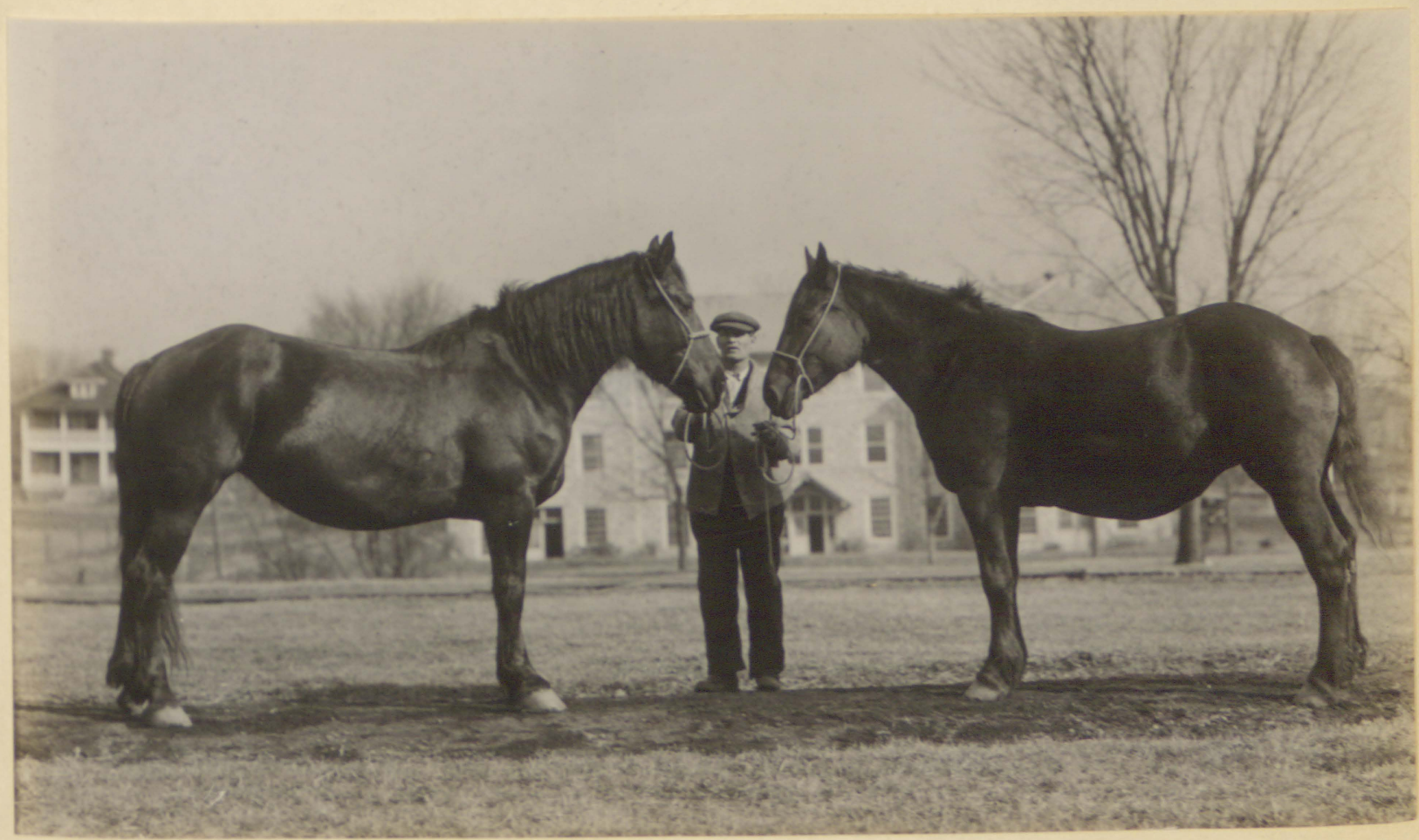

IMMATURE PREGNAITT MARES PRRFORMING AVERAGE WINTER FARM WORK LOT II JOSEPHB, NO. 4, NO SILAGE IOT I MENA, NO. 3, SILAGE 
Tables XXXII and XXXII ${ }^{A}$ present the data for Group $D-c$, Lots I and II, immature pregnant mares performing average winter farm work, Wens, No. 3, and Josephe, No. 4.

Lot I received silage as a part ration. Iot II received no silage.

This pair of three year old pregnant mares were in medium breedirg condition at the beginning of the test. The Initial weight of Lena, No. 3 was 1517 pounds and the initial weight of Josephe, No. 4 was 1567 pounds. Lot I gained 40 pounds and Lot II gained 3 pounds during the test. This team performed a total of 393 hours of work during the test, most of which is classified as heary labor. Niena, No. 3 was bred April 5, 1915 and Josephe, No. 4 was bred if a 31,1915 .

Lot II receired daily .845 pounds of grain and 7.425 pounds of hay more than Iot I. Iot I was fed a daily average of 9.896 pounds of silage.

From the table of digestible nutrients consumed it will be noted that Lot II received 5.350 pounds of dry matter, 1.342 pounds of carbohydrates and .155 pounds of ash per day more than Lot I. Both lots received practically the same quantity of protein and fat.

When reduced to a thousand pound basis, Lot I received da1ly 3.067 pounds of ary matter, .582 pounds of carbohydrates and .082 pounds of ash more than Lot I. Lot I consumed .023 pounds of protein and .01I pounds 
of fat per day in excess of that consumed by Lot II.

There was no noticeable difference in the condition of flesh of these lots at the close of the experimert.

The hair and skin of Lot I was in better condition than the hair and skin of Iot II. 


\section{TABIB XOXIII}

TEED CONSUNGD*AND WEIGHTS*BY TREKCY PERIODS

JOSEPHINE NO. 23

DATS ${ }^{1}$ GRAIN 2 HAY 1915

Doo.17

\% 2470

- 3170

1916

Jan. 770

" 1470

" 2170

n 2870

Fob. 470

- 1170

" 18100

n 25100

Moh. 3100

n 10100

Total $960 \quad 776 \quad 1125.75$

Da1ly

$$
\begin{aligned}
& \begin{array}{l}
\text { Dre. } 11.427 \quad 9.238 \quad 13.402 \\
I_{\text {Corn, oats, bran, } 2-2-1}
\end{array} \\
& 2 \\
& \text { Timothy hay } \\
& 3 \\
& \text { Initial woight } \\
& 4088 \text { in w018ht } \\
& \text { *Pounds }
\end{aligned}
$$

JESS No. 27

2 HAY SILAGE WEIGHT

$3_{1403}$

$70 \quad 34.5 \quad 1395$

$70 \quad 38.5 \quad 1410$

$70 \quad 40.75 \quad 1370$

$70 \quad 40 . \quad 1370$

$70 \quad 46 . \quad 1400$

$70 \quad 36.5 \quad 1360$

$70 \quad 43.5 \quad 1360$

$68 \quad 39 . \quad 1340$

$70 \quad 50.5 \quad 1340$

$\begin{array}{lll}69 & 42.5 & 1350\end{array}$

$66.5 \quad 49.5 \quad 1330$

68. $54.5 \quad 1353$

$\begin{array}{lllll}4_{5} & 1210 & 831.5 & 512.25 & 4_{50}\end{array}$

$\begin{array}{lll}14.405 & 9.900 \quad 6.098\end{array}$ 


\section{TABIR XXXIII}

JOSEPHINE NO. 23

DIGESTIBLE NUTRIETMS CONSUIED*

TOTAL FEED

CONSULED

$1_{\text {Grain }}$
$2_{\text {Hay }}$

Sllage

Total

Av. Da1ly

$$
960.0
$$

776.0

$1125 \cdot 75$
DRY vaTTER PROTEIN

876.199

724.450

401.229

81.118

7.659

17.798

2001.878

$106.575 \quad 1023.560$

23.832

1.269

DIGESTIBLE NUTRIENMS CONSUAED*

PER 1,000 POUNDS II TE WEIGET

motal

Av. Da11y
1373.025

16.346
$73.097 \quad 702.030$

.870

8.258
12.185

CARBOHYDRATES PAT ASH

506.942 288.462 228.156

26.680 7.279 $12.575 \quad 22.087$ $46.534 \quad 89.505$ $.554 \quad 1.066$

JEsS NO. 27

DIGESTIBLR NUMRIEUTS CONSUDTE *

$\begin{array}{lrrrrrr}I_{\text {Grain }} & 1210.0 & 1104.384 & 103.577 & 638.798 & 33.642 & 38.732 \\ 2_{\text {Hay }} & 831.5 & 776.263 & 8.207 & 309.093 & 7.799 & 39.995 \\ \text { S11ag0 } & 512.25 & 182.571 & 8.099 & 103.818 & 5.722 & 10.050 \\ \text { Total } & & 2063.218 & 119.883 & 1051.709 & 47.163 & 88.777 \\ \text { Av. Daily } & & 24.562 & 1.427 & 12.520 & .561 & 1.057\end{array}$

DIGESTIBLE NUTRIENMS CONSUNED*

PER 1,000 POURDS LIVE TEIGHM

Total

AT. Da11y
1508.200

17.955

1.043

$31.916 \quad 61.389$

$.380 \quad .731$

1corn, oats, bran, $2-2-1$

2 Timothy

*Pound 8 
JOSEPHINE, IUUMBER 23

PHOTOGRAPHED AT THE BEGINIIING OF EXPERTMEIT, DECBMBER 18, 1915

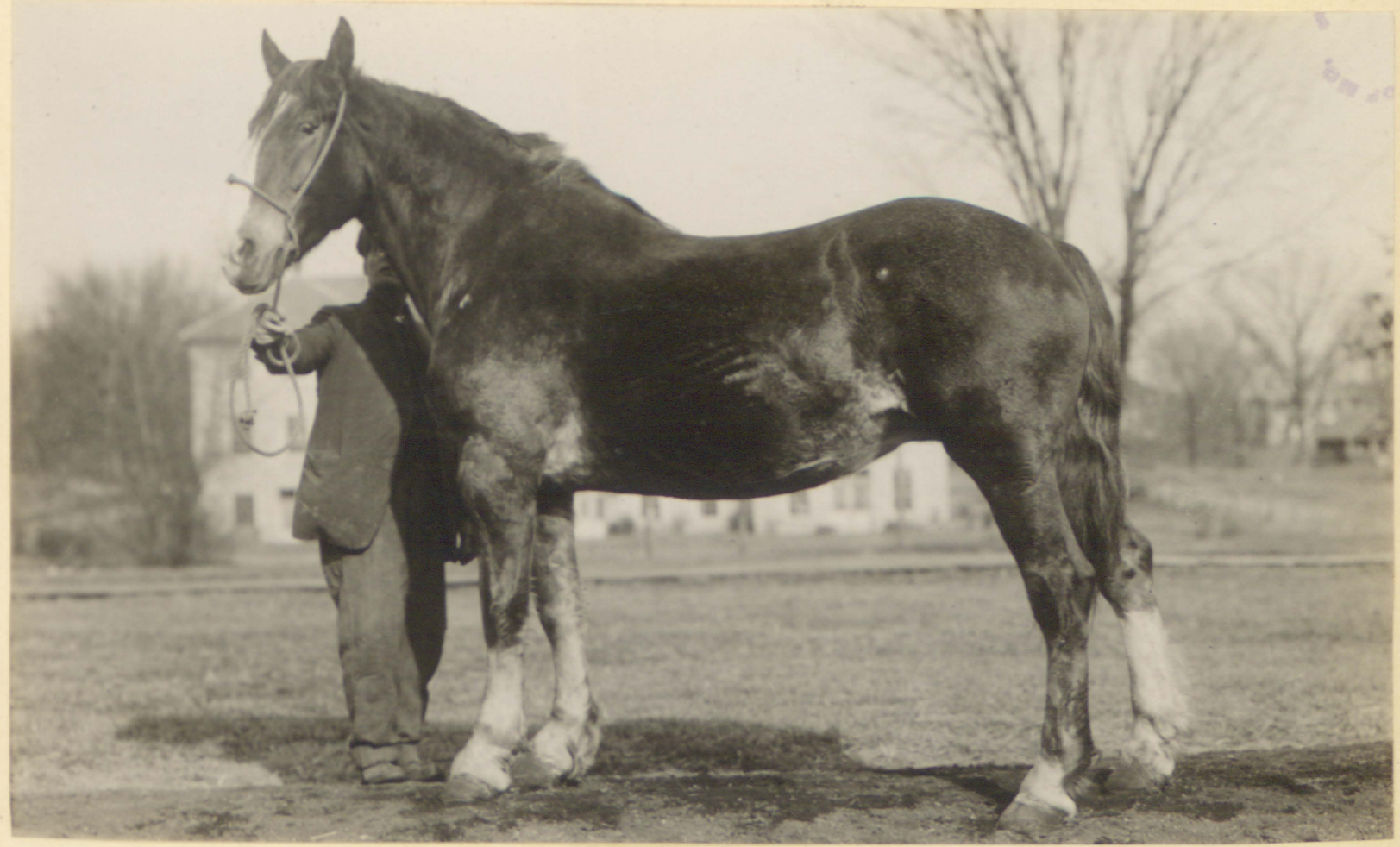

PHOTOGRAPHED AT THE CLOSE OF THE BXPERINESNT, MARCH 11, 1916

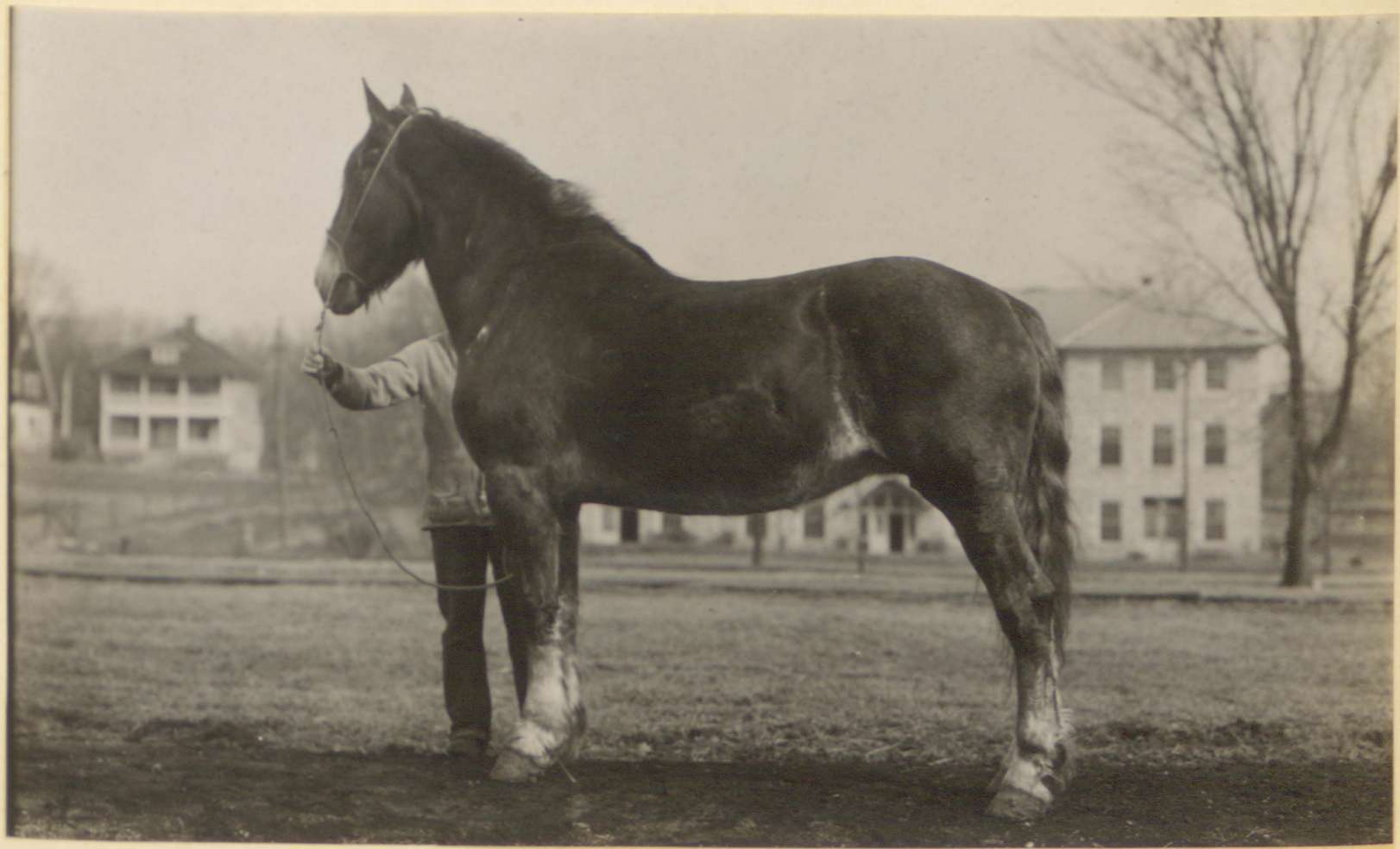


JESS, TUMBBHR 27

PHOTOGRAPHED AT THE BEGINIING OF EXPER IMEINT, DECGMBBR 18,1915

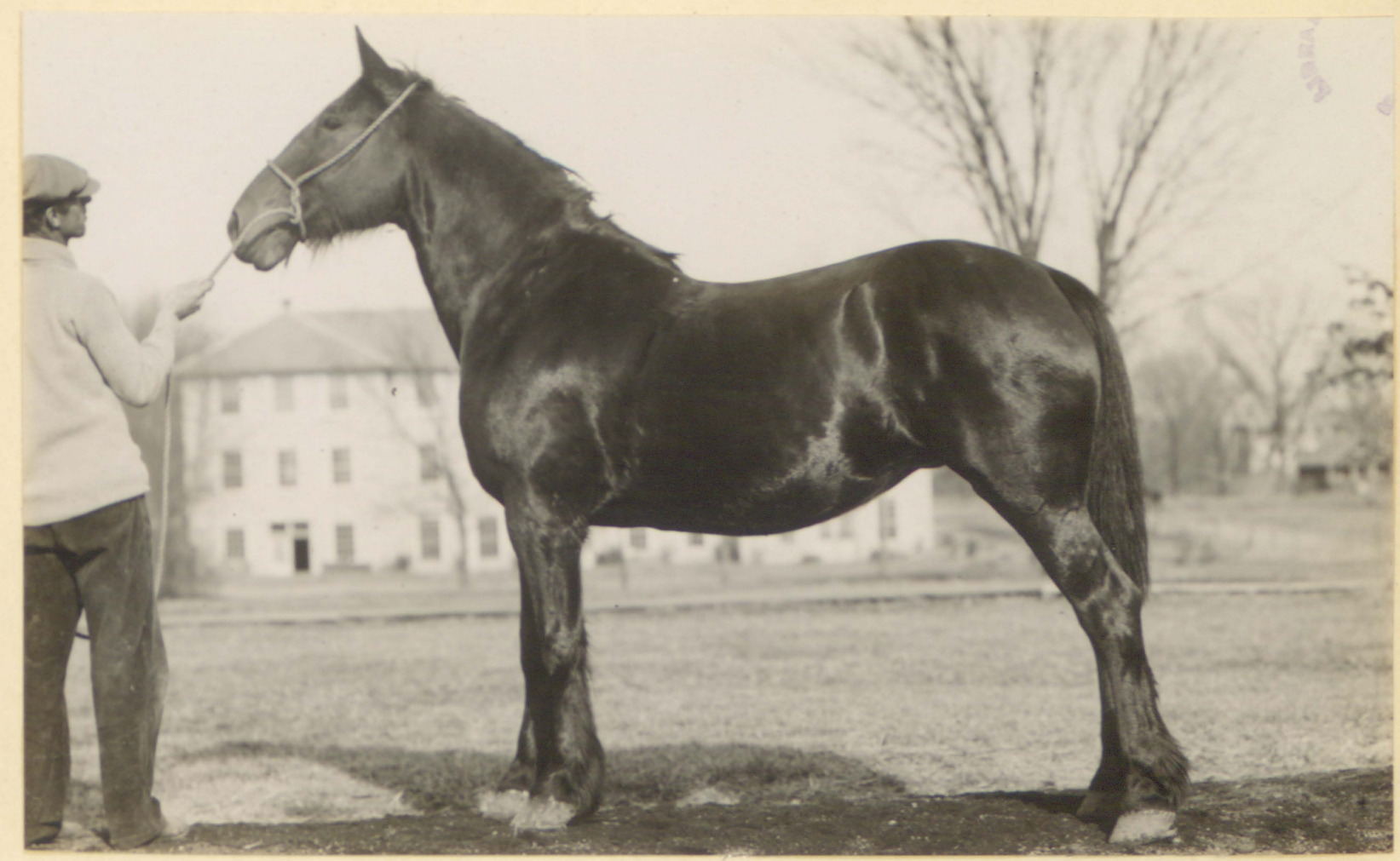

PHOTOGRAPHED AT THE CLOSE OF THR EXPERIMENT, MARCH 11,1916

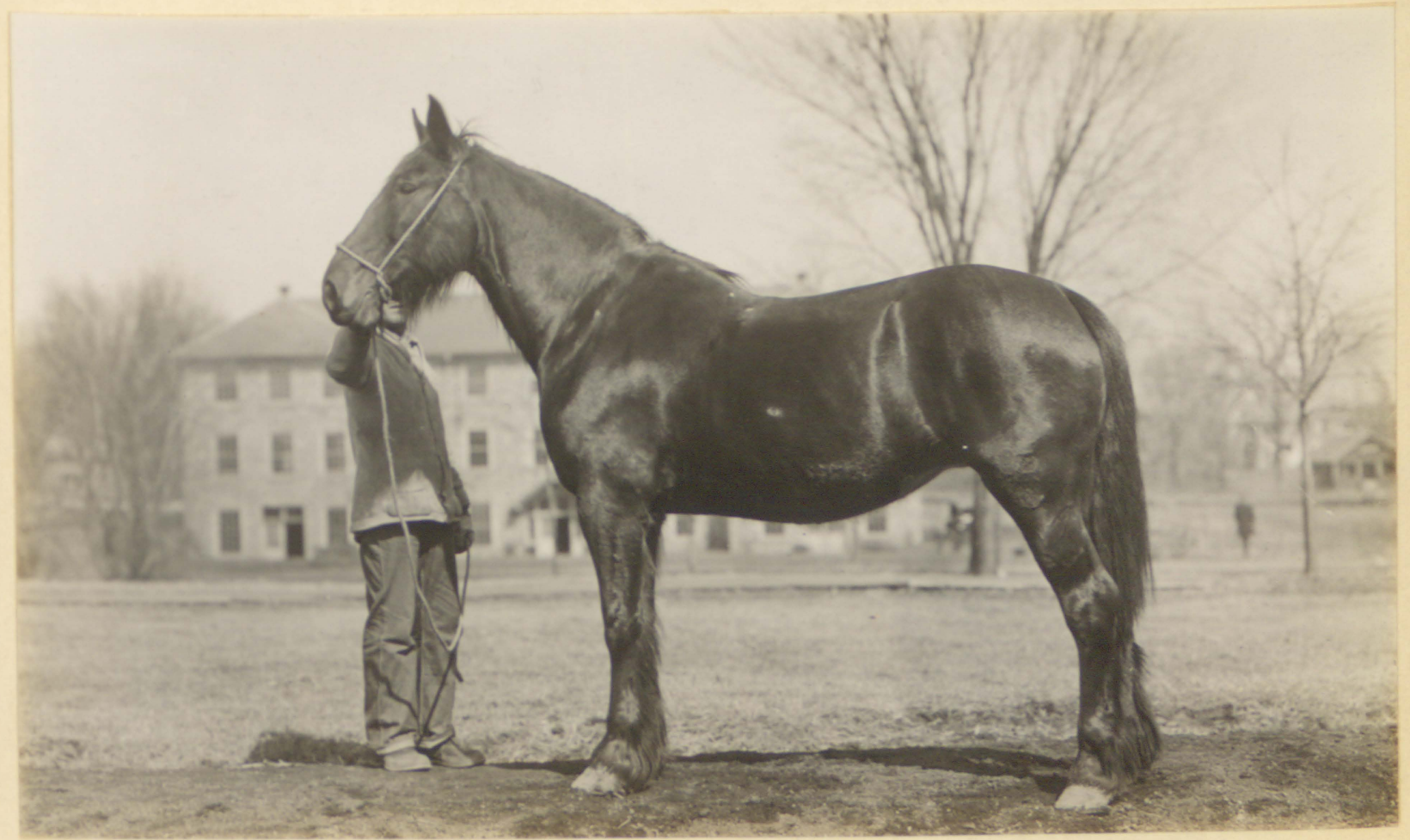


The horses Josephine, No. 23, Jim, No. 25, Jess, No. 27. Magna, No. 29 and Lady Adrance, No. 31, were fed silage as a part ration.

There was no way of dividing these horses into lots for comparison.

They were fed silage as a part ration in order to get aditional data and information on silage as a part ration for horses of various ages and under varying conditions.

Draft horses Josephine, No. 23, Jim, No. 25 and Jess, No. 27 were fed a ration of grain, timothy hay and silage. Iight horses, Magna, No. 29, and Lady Advance, No. 31, were fed a ration of grain, alfalfa hay and silage.

Tables XXXIII and $\operatorname{XXXIII}^{A}$ present the date for Josephine, No. 23, idle registered Percheron mare not in foal, and, Jes8, No. 27, open grade draft mare performing delivery work. Both horses were led grain, timothy hay and silage. Josephine, No. 23, was in poor condition at the beglnning of the test. She was lame, having run a nail in her foot some time previous to the beginning of the feeding period. Jess, No. 27, was in medium working condition when the test began.

Josephine, No. 23, maintained her weight throughout the test on a ration of 11.427 pounds of grain, 9.238 pounds of hay and 13.402 pounds of silage per day.

Jess, No. 27, lost 50 pounds in weight during the test. She was fed a dally arerage of 14.405 pounds of grain, 
9.9 pounds of hay and 6.098 pounds of silage. It was impossible to keep a record of the work hours performed, but she was in harness from 7:30 A. M. to 5:30 P. M. practically every day.

Per thousand pounds live weight, Josephine, No. 23, consumed daily 16.346 pounds of dry matter, .870 pounds of protein, 8.258 pounds of carbohydrates, .380 pounds of fat and .731 pounds of ash. Jess, No. 27, received per thousand pounds live weight, 17.955 pounds of dry metter, 1.043 pounds of protein, 9.152 pounds of carbohydrates, .410 pounds of fat, and .772 pounds of ash per day.

The hair and skin of these mares at the close of the experiment indicated good thrift. 


\section{TABIE XXXIV}

FEWD CONSUMGI* AND WEIGHMS* BY WEEKCY PERIODS JIM NO. 25

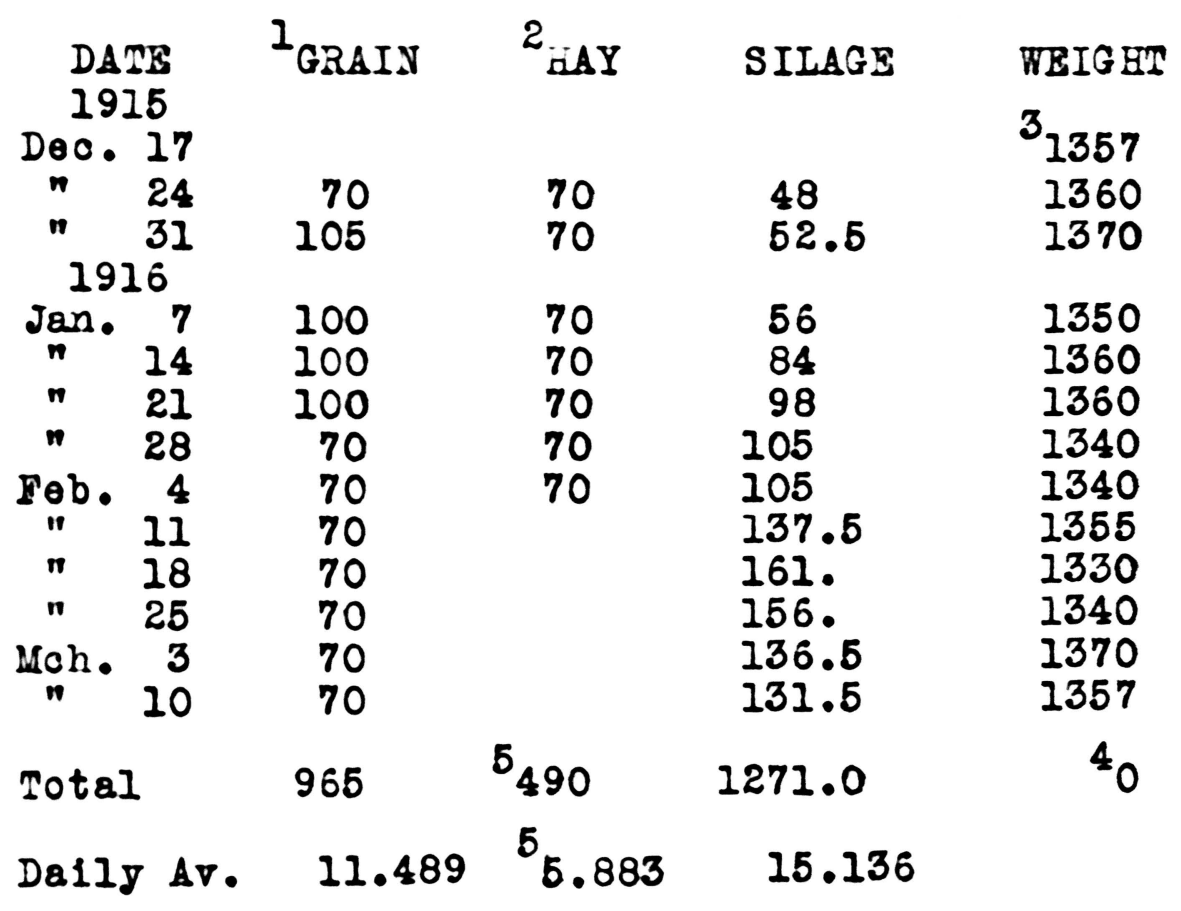

1'corn, oats, bran, $2-2-1$

$2_{\text {Timothy hay }}$

3 Initial woight

4 Gain or 1088 in weight during feeding period

5 red hay only seren weeks

*Pound 8 
TABIE XXXIVA

DIGESTIBIE NUTRIEITSS CONSUNED*

JIM NO. 25

TOTAL FEBD COHSURDR

$I_{\text {Grain }}$
$2_{\text {Haj }}$
S1lage
Total
Ar. Daily

965.0
490.0
1271.0

DRY MATTER PROTEIN

CARBO-

HYDRATES FAT ASH

880.583

457.449

452.997

82.701

509.246

$4.836 \quad 182.148$

20.095

257.594

26.849

4.596

14.197

24.937

31.039

23.569

1791.029

107.632

948.988

21.322

1.281

11.298

.543

79.545

DIGESTIBLE NUTRIENTS CONSURED*

PER 1,000 POUNDS II VE WEIGHT

Total

Ar. Daily
1323.746

15.759
79.55

.947
701.395

8.350
58.792

.700

\footnotetext{
${ }^{1}$ Corn, oats, bran, $2-2-1$

2 TImothy

*Pounds
} 
JIM, NUMBER 25

PHOTOGRAPHED AT THE BEGIMIING OF BXPERTMENT, DECHUBER 18, 1915

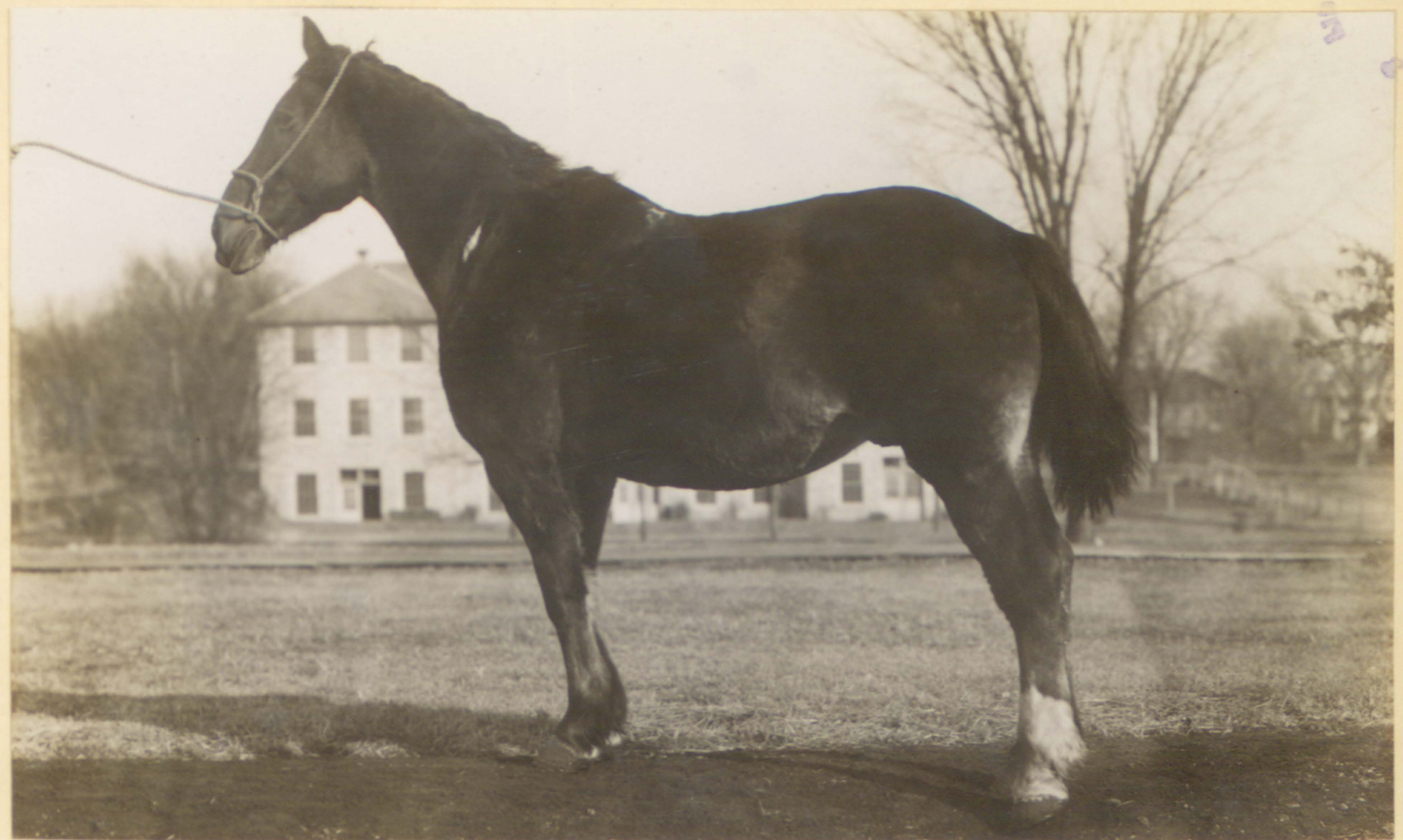

PHOTOGRAPHED AT THE CLOST OF THE BXPERIMENT, MARCH 11, 1916

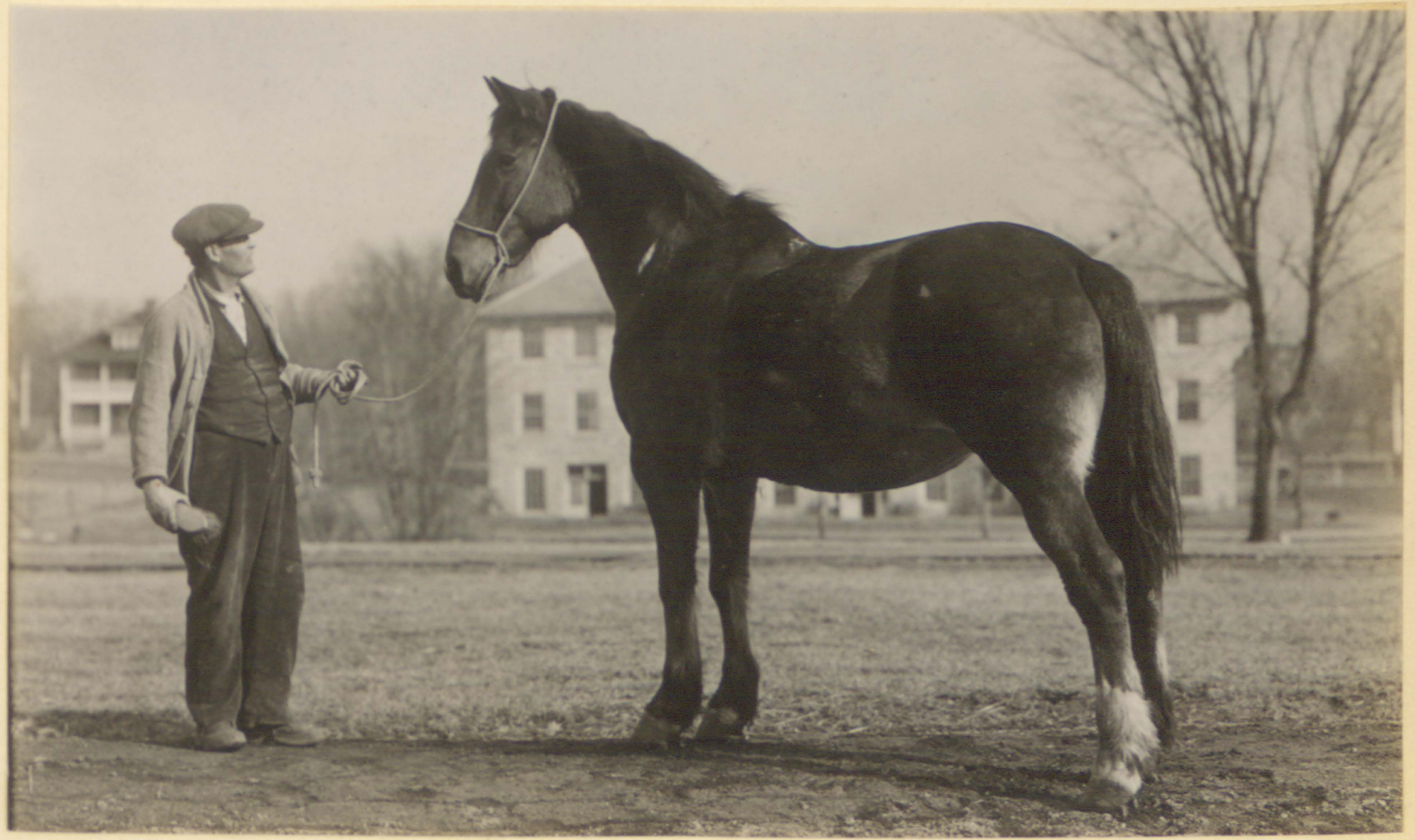

MATURE IDLE GELDING

SIIAGE 


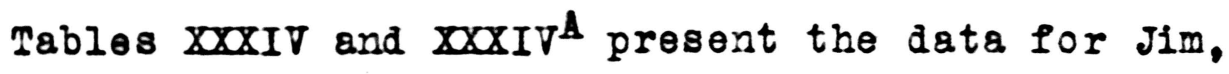
No. 25, idle draft gelding. He was fed a ration of grain, hay and 81 lage.

His initial and final woights were exactly the same. He was maintained for 12 weeks on a ration of 965 pounds of grain, 490 pounds of hay and 1271 pounds of silage.

During the first seren weeks of the test he was fed grain, hay and sllage. The last five weeks of the test he was fed only grain and silage.

He consumed per thousand pounds live weight a daily average of 15.759 pounds of dry matter, .947 pounds of protein, 8.35 pounds of carbohydrates, .402 pounds of fat and .7 pounds of ash.

He was in good thrifty condition at the close of the test. 
TABIE XXXV

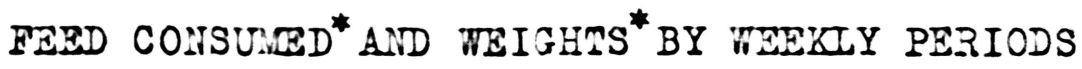

LAGITA NO. 29 LADY ADTANCE NO. 31

DATE $1_{\text {GRAIN }} 2_{\text {HAY }}$ SILAGE WEIGHI $1_{\text {GRAIN }} 2_{\text {HAY }}$ SILAGT HEIGHT 1915

Dec.17

" 2428

" 3128

1916

Jan. 728

" 2128

" 2828

Feb. 428

" 1128

" 1828

" 2528

Moh. 328

" $10 \quad 28$

Total $336 \quad 420$

35

35

35

35

35

35

35

35

35

\section{$3_{1043}$}

$46.5 \quad 1060$

50.251060

56

1050

$55 \quad 1050$

46.751060

$42.75 \quad 1060$

$50.75 \quad 1015$

$48.75 \quad 1030$

51. 1030

47.51020

55. $\quad 1030$

56. 1027

28

35

35

56

56

56

56

56

56

56

56

56

56

${ }^{4} 16$

676

35

35

35

35

35

35

35

35

35

35

$\begin{array}{lr} & 3_{1017} \\ 45.5 & 1000 \\ 45 . & 985\end{array}$

605.25

420

47.5

56.

84.

84.

84.

84.

84.

84.

84.

80 .

862

$\begin{array}{lll}7.323 & 5 & 10.262\end{array}$

Daily Ave.

$4 \quad 5 \quad 7.205$

10.262

${ }^{1}$ Corn, oats, bran, 2-2-1

2

Alfalfa hay

3

Initial weight

4

Los8 in weight

*Pounds 


\section{TABIE $X \times V^{A}$ \\ MAGNA NO. 29}

DIGESTIBIE NUMRIENTS COMSU.EE

TOTAL FEED COHSU.MD

Iarain
2Hay
Silage
Total
AT. Daily

Total

Av. Da1Iy
DRY MATMER PROTEIN HYRBATES FAT ASH 303.672 391.301 232.558

927.531

11.042

$$
\begin{array}{rrrr}
28.763 & 177.378 & 9.342 & 10.758 \\
44.650 & 166.459 & 1.693 & 38.338 \\
9.569 & 122.666 & 6.761 & 11.875 \\
82.982 & 466.503 & 17.796 & 60.971 \\
.988 & 5.554 & .212 & .726
\end{array}
$$

DIGESTIBIE NUTRIENTS CONSUEED*

PER 1,000 POUNDS II TE WEIGHS

$\begin{array}{rrrrr}891.000 & 79.714 & 448.130 & 17.105 & 58.570 \\ 10.607 & .949 & 5.335 & .204 & .697\end{array}$

IADY ADVAITCE NO. 31

DIGESTIBLE NUMRIEITS CONSUIRD*

$\begin{array}{lrrrrrr}I_{\text {Grain }} & 616.0 & 560.071 & 52.691 & 325.789 & 17.119 & 19.658 \\ \text { Z Hay }_{\text {HIlage }} & 420.0 & 391.301 & 44.650 & 166.459 & 1.693 & 38.338 \\ \text { S1l.0 } & 862.0 & 307.225 & 13.628 & 174.702 & 9.629 & 16.912 \\ \text { Total } & & 1258.597 & 110.969 & 666.950 & 28.441 & 74.908 \\ \text { Ar. Da11y } & & 14.983 & 1.321 & 7.940 & .339 & .892\end{array}$

DIGESTIBLE NUMRIENTS CONSURDED

PER 1,000 POUNDS IIVE WEIGHT

Totan

Av. Da1ly
1263.652

15.044

111.415

669.62928 .558

75.209

$$
1.326
$$

7.978

.340

.895

${ }^{1}$ corn, oats, bran, 2-2-1

2 Alfalfa

*Pound 8 
MAGNA, NUMBER 29

PHOTOGRAPHTD AT THE BEGINIING OF EXPERIMENT, DECHIBER 18, 1915

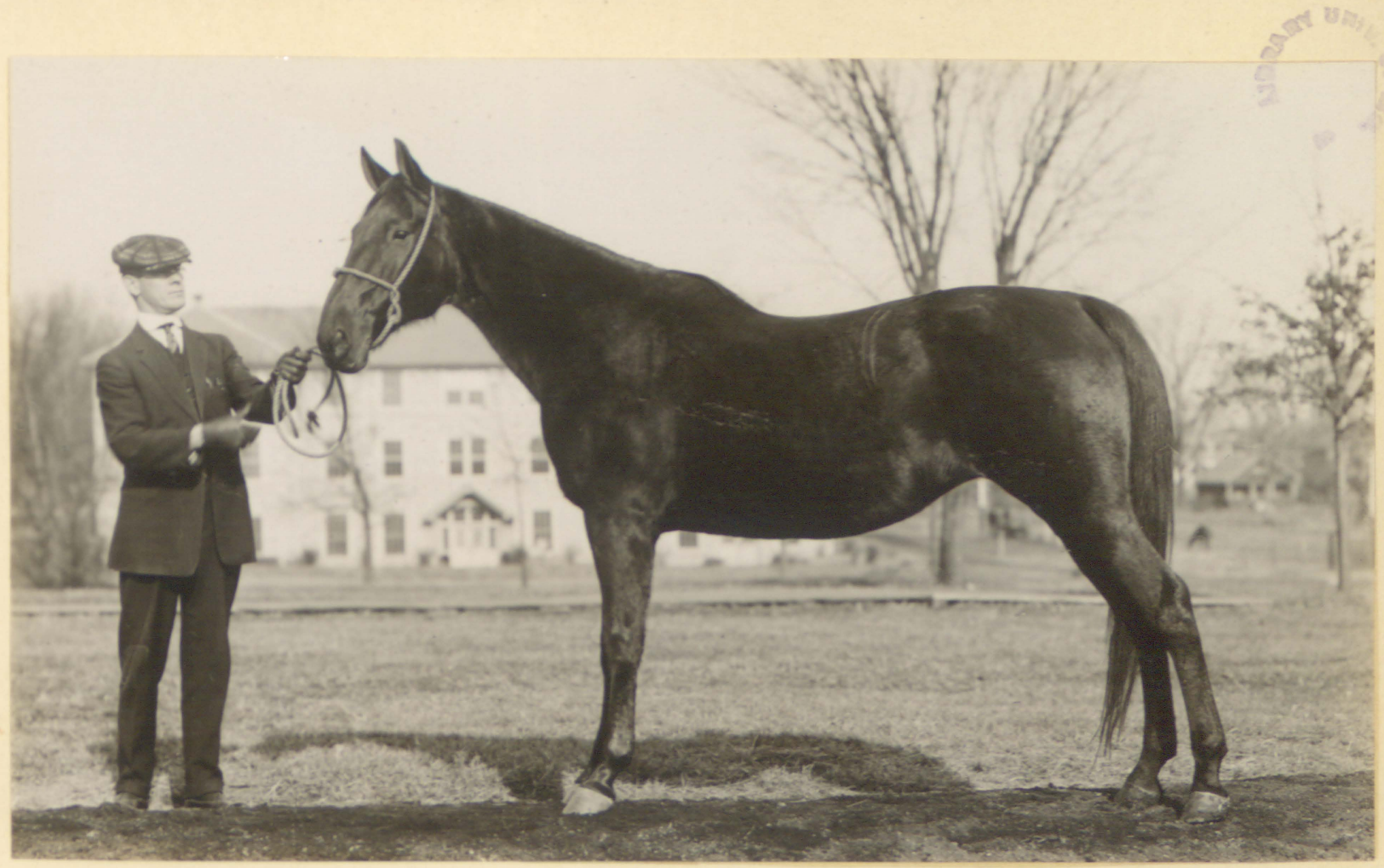

PHOTOGRAPHED AT THE CLOSE OF THE EXPERIMENT, MARCH 11,1916

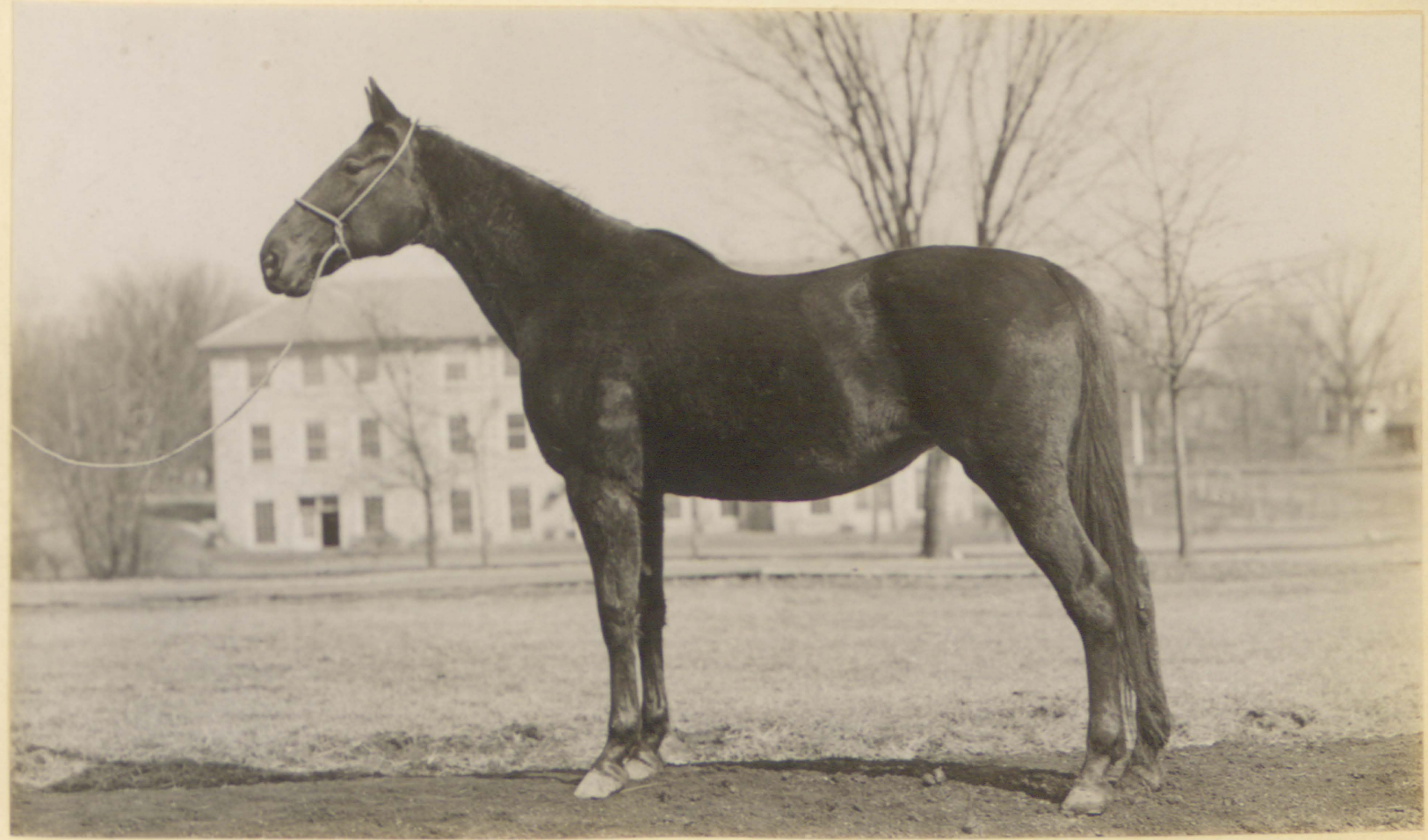


IADY ADVAITCE, NUMBER 31

PHOTOGRAPHED AT THE BEGINNING OF EXPERIMENT, DECEMBER 18, 1915

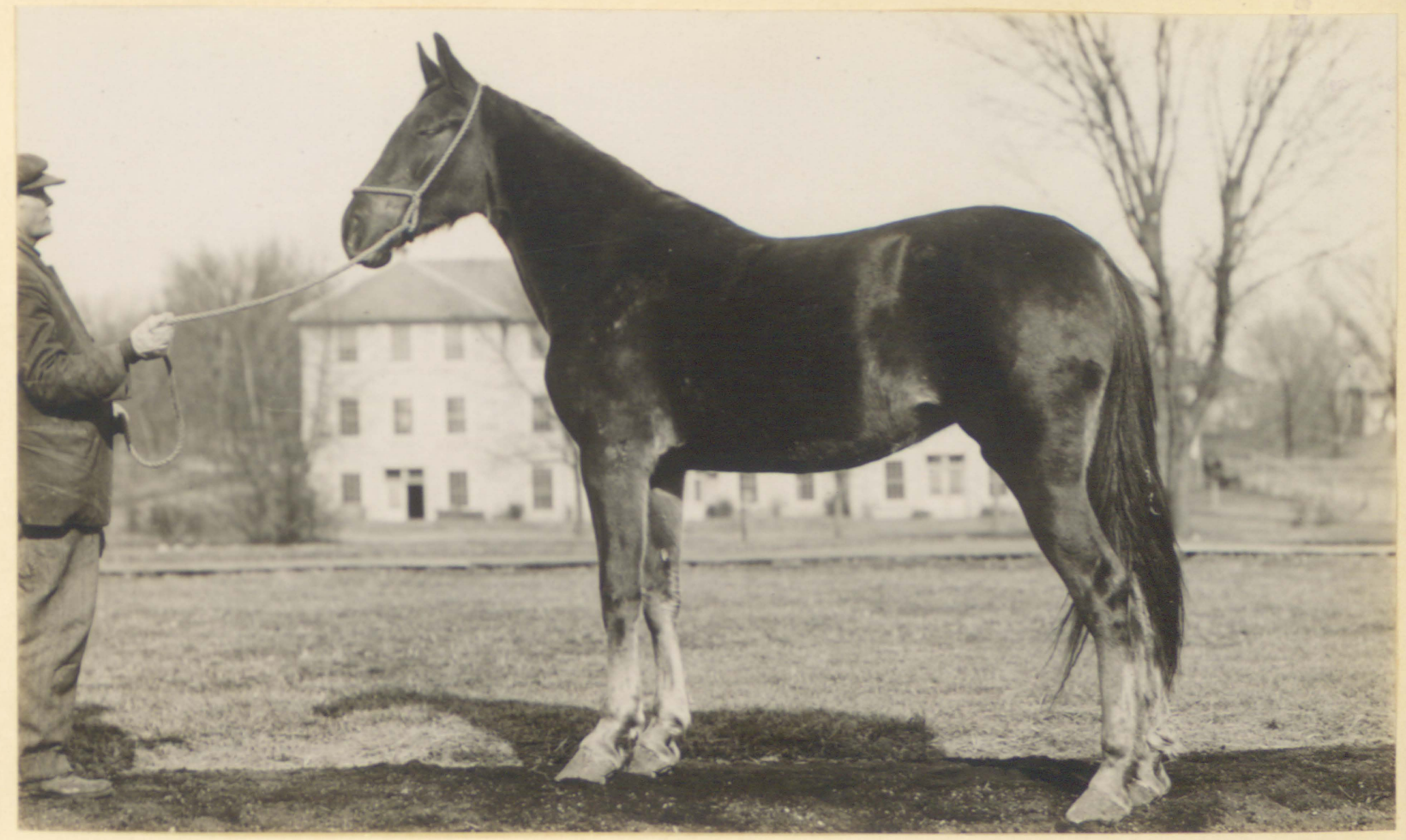

PHOTOGRAPHED AT THE CLOSE OF THE EXPERIMENT, MARCH 11, 1916

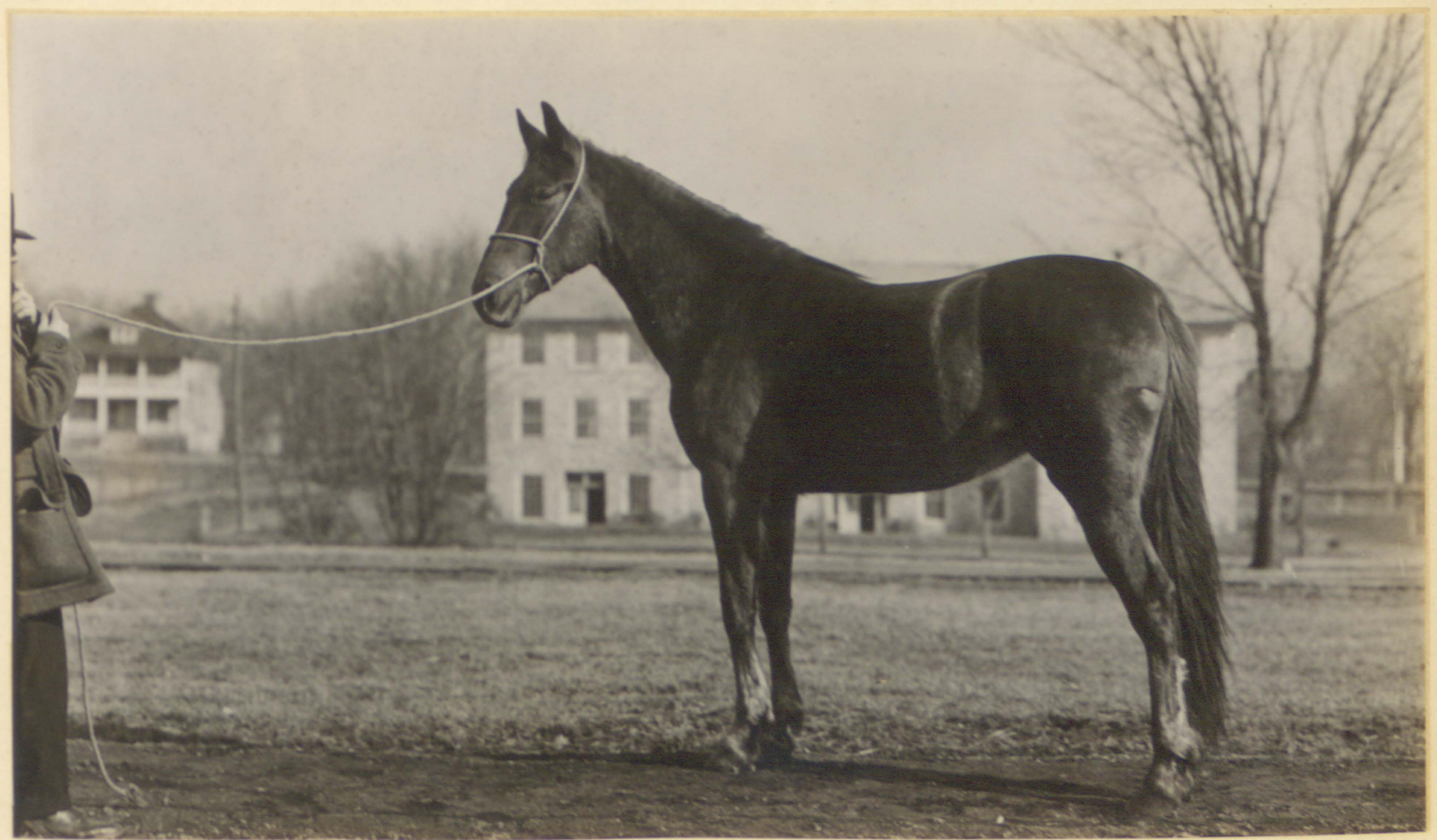

GROWING STANDARD BRID MARB. SHE WAS USED FOR LIGHT DRIVING. SIILAGR 
Tables $\mathrm{XXV}$ and $X 0 X V^{\mathrm{A}}$ present the data for Magna, 110. 29, 1dle saddle mare not in foal, and two year old standard bred 11lly. Lady Adrance, No. 31.

Both horses were fed grain, alfelfa hag and silage. Magna, No. 29, was in medium condition and Lady Adrance, No. 31 in excellent condition at the beginning of the test.

Magna, No. 29, lost 16 pounds in weight and Lady Advance, Iro. 31, lost 12 pounds while on the test. Magna, No. 29, was fed a daily ration of 4 pounds of grain, 5 pounds of alfalfa hay and 7.205 pounds of silage. Lady Adrance, No. 31 was fed a daily ration of 7.333 pounds of grain, 5 pounds of hay and 10.262 pounds of silage. She was driven a little each day during the test.

The digestible nutrients consumed by Hagna, No. 29, when reduced to a thousand pound basis were, 10.607 pounds of dry matter, .949 pounds of protein, 5.335 pounds of carbohjdrates, .204 pounds of fat and .697 pounds of $88 \mathrm{~s}$ per daj. Per thousand pounds live weight, Lady Adranoe consumed da1ly 15.044 pounds of dry matter, 1.326 pounds of protein, 7.972 pounds of carbohydrates, .340 pounds of fat and .895 pounds of $88 h$.

These mares were in thrifty condition at the end of the test. 
TABLE XXXVI

SUACARY OF TOMAL FEED CONSUIED*DURING 1915-1916 TEST IOT I

NAing

Jane

Martha 2nd

Satel1to

Cap

Folibreo

Filly rool

Joscelin

stud Foal 2

Sport

Mena

\section{IUNABER}

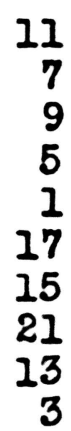

GRAIN

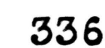

336

336

1200

1200

567

840

393

392

1200
BAY

7420.0

7418.5

7245 .

8741.25

8775.5

7420 .

7470 .

7415.5

7411.5

8725.5

SILAGE

742.00 1140.25 1336.25

1137.75

1130.50

335.75

1142.75

381.50

906.75

831.25

\section{IOI II}

J111

Ruth MoDonald

Iona Rex

Maok

Forgette

Filiy $\mathrm{Foal}^{3}$

Draft Golding 4

Stud Foal 5

Ruth Squirrel

Josephe

Jos ophine

Jim

Jes8

Lagna

Lady Advanco
12

8

10

6

18

16

22

14

4

23

25

27

29

31 $\begin{array}{cl}336 & 71218 . \\ 336 & 71218 . \\ 336 & 71215 . \\ 1271 & 81386.5 \\ 1271 & 81401.5 \\ 567 & 7770 . \\ 840 & 71218 . \\ 390 & 7445.75 \\ 392 & 71202.50 \\ 1271 & 81455 .\end{array}$

960

965

1210

336

616
8776
8490
8831.5
7820
7420
1125.75

1271.00

512.25

605.25

862 .

*Pounds

$I_{\text {Dam, Josephine, Sire, Honorable }}$

2Dam, Ruth MoDonald, Sire Astral King

$3_{\text {Dam, Forgette, Sire, Honorable }}$

${ }^{4}$ Dam, Finette, Sire, Gardiner

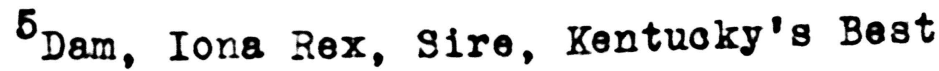

${ }^{6}$ Corn, oats, bran, 2-2-1 7 alfalfa 
TABIR XXXVII

SURDIARY OF AVERAGE FEED CONSUIED* PER DAY DURIIIG 1915-1916 TEST LOT I

\section{NA:T}

June

Martha 2nd

Satelite

Cap

Felibreo

Filly ForI

Joscelin

Stud Foal 2

Sport

Liena
NU.IBER

11
7
9
5
1
17
15
21
13
3

JiII

Ruth MeDonald

Iona Rex

Lack

Forgette

Filis Foor ${ }^{3}$

Draft Gelding 4

Stud Foals

Ruth Squirrel

Josephe

Josephine

Jim

Jess

Magna

Lady Advanoo

\section{GRAIN}

4.0

4.0

4.0

14.286

14.286

6.75

10.00

4.679

4.667

14.286
HAY

75.0

4 .

88.824

89.226

75.0

75.667

7.946

7.900
8.637
SIIAGE

8.833

13.574

15.908

13.545

13.452

3.997

13.604

4.542

10.795

9.896

LOT II

$\begin{array}{rcl}12 & 4.0 & 714.5 \\ 8 & 4.0 & 714.5 \\ 10 & 4.0 & 714.64 \\ 6 & 15.131 & 816.506 \\ 2 & 15.131 & 816.685 \\ 18 & 6.75 & 79.167 \\ 16 & 10.00 & 714.500 \\ 22 & 4.643 & 75.307 \\ 14 & 4.667 & 714.315 \\ 4 & 15.131 & 817.321\end{array}$

*Pound 8

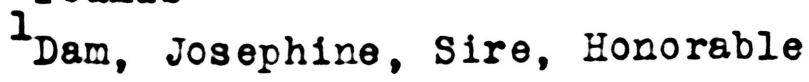

2Dam, Ruth MoDonald, Sire, Astral King

3 Dam, Forgette, Sire, Honorable

4Dam, finette, sire, Gardiner

5

Dam, Iona Rex, Sire, Kentucky's Best

${ }^{6}$ corn, oats, bran, $2-2-1$ ialfalfa 
Tables XXXVI and XXXVII present a summary of the total grain and silage and the average grain, hay and silage consuned per day by all the horses during the 1915-1916 test. The horses in Lot I recoived silage as a part ration. The horses in Lot II received no silage.

The horses in Lot I received an average of 8.090 pounds of grain, 6.218 pounds of hag and 10.815 pounds of 81 lage per daj.

The horses in Lot II consumed a daily average of 8.345 pounds of grain and 13.745 pounds of hag during the test. The horses in Iot I made a total gain of 262 pounds and the horses in Iot II made a total gain of 638 pounds during the feoding period.

The fire horses, Josephine, No. 23, Jim, No. 25, Jes8, No. 27, Magna, No. 29, and Lady Advance, No. 31, recelved a daily average of 9.731 pounds of grain, 7.828 pounds of hay and 10.421 pound of silage during the test.

Comparing the average feed consuned by both lots it will be noted that an average of 10.815 pounds of silage fed to horses in Lot I replaced .255 pounds of grain and 7.527 pounds of hay in the ration.

The horses in Lot I did not gain as much as the horses in Lot II. The average of the horses in Lot I showed as much thrift as the average of horses in Lot II at the close of the test. 


\section{TABIS XXXVIII}

SUACLARY OF TOMAL DIGESTIBIE NUTRIENTS COMSURED*DURIMG 1915-1916 TEST LOT I

NALES NO. DRY MATTER PROTEII CARBO FYDRATES FAT ASH

$\begin{array}{lrrrrrr}\text { June } & 11 & 959.429 & 85.144 & 494.218 & 19.323 & 63.654 \\ \text { Marthe 2nd } & 7 & 1099.973 & 91.281 & 534.336 & 28.766 & 71.331 \\ \text { Satelite } & 9 & 1088.184 & 75.935 & 545.297 & 25.255 & 59.339 \\ \text { Cap } & 5 & 2192.491 & 128.037 & 1139.640 & 52.872 & 96.397 \\ \text { Felibree } & 1 & 2221.237 & 128.240 & 1150.615 & 53.032 & 97.869 \\ \text { F1lly Foal } & 17 & 1029.472 & 98.463 & 533.913 & 21.201 & 63.027 \\ \text { Joscel1n } & 15 & 1617.443 & 140.581 & 863.709 & 38.029 & 92.764 \\ \text { Stud Foal } 2 & 21 & 881.772 & 83.817 & 449.532 & 16.811 & 57.948 \\ \text { Sport } & 13 & 1064.338 & 91.611 & 553.870 & 22.679 & 67.857 \\ \text { Hena } & 3 & 2068.548 & 123.029 & 1071.666 & 49.230 & 89.626\end{array}$

IOT II

\begin{tabular}{|c|c|c|c|c|c|c|}
\hline $\begin{array}{l}\text { Jill } \\
\text { Ruth MoDonald } \\
\text { Iona Rex } \\
\text { Wack } \\
\text { Forgette } \\
\text { F1liy Joal } \\
\text { Draft Gelding } 4 \\
\text { Stud Foal5 } \\
\text { Ruth Squirrel } \\
\text { Josepho }\end{array}$ & $\begin{array}{r}12 \\
8 \\
10 \\
6 \\
2 \\
18 \\
16 \\
22 \\
14 \\
4\end{array}$ & $\begin{array}{r}1438.446 \\
1438.446 \\
1435.651 \\
2454.142 \\
2468.145 \\
1234.892 \\
1901.454 \\
771.248 \\
1478.124 \\
2518.091\end{array}$ & $\begin{array}{r}158.249 \\
158.249 \\
157.930 \\
122.451 \\
122.600 \\
130.364 \\
201.394 \\
80.752 \\
161.366 \\
123.128\end{array}$ & $\begin{array}{r}660.108 \\
660.108 \\
658.919 \\
1186.474 \\
1192.050 \\
604.578 \\
926.185 \\
386.564 \\
683.596 \\
1174.374\end{array}$ & $\begin{array}{l}14.239 \\
14.239 \\
14.238 \\
48.337 \\
48.478 \\
18.861 \\
28.243 \\
12.635 \\
15.739 \\
48.980\end{array}$ & $\begin{array}{r}121.937 \\
121.937 \\
121.663 \\
107.326 \\
108.047 \\
88.388 \\
138.073 \\
53.140 \\
122.269 \\
102.621\end{array}$ \\
\hline $\begin{array}{l}\text { Josephine } \\
\text { Jim } \\
\text { Jess } \\
\text { Lagna } \\
\text { Iady Advance }\end{array}$ & $\begin{array}{l}23 \\
25 \\
27 \\
29 \\
31\end{array}$ & $\begin{array}{r}2001.878 \\
1791.029 \\
2063.218 \\
927.531 \\
1258.597\end{array}$ & $\begin{array}{r}106.575 \\
107.632 \\
119.883 \\
82.982 \\
110.969\end{array}$ & $\begin{array}{r}1023.560 \\
948.988 \\
1051.709 \\
466.503 \\
666.950\end{array}$ & $\begin{array}{l}46.534 \\
45.642 \\
47.163 \\
17.796 \\
28.441\end{array}$ & \\
\hline
\end{tabular}

${ }^{I}$ Dam, Josephine, Sire, Honorable

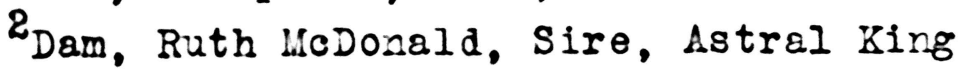

3

Dam, Forgette, Sire, Honorable

4 Dam, Finette, Sire, Gardiner

5 Dam, Iona Rex, Sire, Kentucky's Best

*Pound 8 
TABIR XXXIX

SUDCLARY OF AVERAGE DAIIY DIGESTIBIE NUTRIE:TS CONSU.RD DURIIG 1915-1916 TEST

\author{
LOM I
}

MAIE HO. DRY WATTER PROMEIN CARBO FAT ASH

$\begin{array}{lrrrrrr}\text { June } & 11 & 11.422 & 1.014 & 5.884 & .230 & .758 \\ \text { Martha 2nd } & 7 & 13.095 & 1.087 & 6.361 & .283 & .849 \\ \text { Satelite } & 9 & 12.002 & .904 & 6.492 & .265 & .706 \\ \text { Cap } & 5 & 26.101 & 1.524 & 13.567 & .629 & 1.148 \\ \text { Folibree } & 1 & 26.443 & 1.527 & 13.698 & .631 & 1.165 \\ \text { Filly Foal } & 17 & 12.255 & 1.172 & 6.355 & .252 & .750 \\ \text { Joscelin } & 15 & 19.255 & 1.674 & 10.282 & .453 & 1.104 \\ \text { Stud Fool2 } & 21 & 10.497 & .999 & 5.352 & .200 & .690 \\ \text { Sport } & 13 & 12.671 & 1.090 & 6.594 & .270 & .808 \\ \text { Hena } & 3 & 24.626 & 1.465 & 12.758 & .586 & 1.067\end{array}$

\title{
IOT II
}

\begin{tabular}{|c|c|c|c|c|c|c|}
\hline $\begin{array}{l}\text { Jill } \\
\text { Ruth MoDonald } \\
\text { Iona Rex } \\
\text { Mrok } \\
\text { Forgette } \\
\text { Filig Foal } 3 \\
\text { Draft Gelding } 4 \\
\text { Stud Foal } \\
\text { Ruth Squirrel } \\
\text { Josephe }\end{array}$ & $\begin{array}{r}12 \\
8 \\
10 \\
6 \\
2 \\
18 \\
16 \\
22 \\
14 \\
4\end{array}$ & $\begin{array}{r}17.124 \\
17.124 \\
17.091 \\
29.216 \\
29.299 \\
14.701 \\
22.636 \\
9.182 \\
17.597 \\
29.976\end{array}$ & $\begin{array}{l}1.884 \\
1.884 \\
1.880 \\
1.458 \\
1.460 \\
1.552 \\
2.398 \\
.961 \\
1.921 \\
1.466\end{array}$ & $\begin{array}{r}7.858 \\
7.858 \\
7.844 \\
14.125 \\
14.191 \\
7.197 \\
11.026 \\
4.602 \\
8.138 \\
14.100\end{array}$ & $\begin{array}{l}.170 \\
.170 \\
.170 \\
.575 \\
.577 \\
.255 \\
.336 \\
.150 \\
.187 \\
.583\end{array}$ & $\begin{array}{r}1.452 \\
1.452 \\
1.448 \\
1.278 \\
1.286 \\
1.052 \\
1.644 \\
.633 \\
1.456 \\
1.222\end{array}$ \\
\hline $\begin{array}{l}\text { Josephine } \\
\text { Jim } \\
\text { Jess } \\
\text { Jagna } \\
\text { Iady Advanoe }\end{array}$ & $\begin{array}{l}23 \\
25 \\
27 \\
29 \\
31\end{array}$ & $\begin{array}{l}23.832 \\
21.322 \\
24.562 \\
11.042 \\
14.983\end{array}$ & $\begin{array}{l}1.269 \\
1.281 \\
1.427 \\
.988 \\
1.321\end{array}$ & $\begin{array}{r}12.185 \\
11.298 \\
12.520 \\
5.554 \\
7.940\end{array}$ & $\begin{array}{l}.554 \\
.543 \\
.561 \\
.212 \\
.339\end{array}$ & $\begin{array}{r}1.066 \\
.947 \\
1.057 \\
.726 \\
.892\end{array}$ \\
\hline
\end{tabular}

$1_{\text {Dam, Josephine, Sire, Honorable }}$

¿Dam, Ruth WeDonald, Sire, Astral King

3 Dam, Forgette, Sire, Honorable

4 Dam, Finette, Sire, Gardiner

5 Dam, Iona Rex, Sire, Kentucky's Best

*Pounds 
Tables XXXVIII and XXXVIX present a summary of the total digestible nutrients and the average dailg digestible nutrients in the 1915-1916 trial.

Lot I received silage as a part ration. Lot II was fed no silage.

The horses in this test received on an average the following nutrients per day:-

Dry Natter Protein Carbohydrates Fat Ash

\begin{tabular}{|c|c|c|c|}
\hline $\begin{array}{l}\text { Lot I } \\
\text { Lot II }\end{array}$ & $\begin{array}{l}16.837 \\
20.395\end{array}$ & $\begin{array}{l}1.246 \\
1.833\end{array}$ & $\begin{array}{l}8.734 \\
9.559\end{array}$ \\
\hline
\end{tabular}

Josephine, No. 23, Jim, No. 25, Jess, No. 27, Nagna, 110. 29 and Lady Advance, 10.31 , received per day an average of 19.148 pounds of dry matter, 1.257 pounds of protein, 9.895 pounds of fat and .938 pounds of ash.

This table shows that the horses in Lot I received an average of 3.558 pounds of dry matter .587 pounds of protein, .825 pounds of carbohydrates and .388 pounds of ash less than Lot II. Lot I recelved .063 pounds of fat per day more than Lot II. 
TABIE XXXX

SURMARY OF TOMAL DIGESTIBIE NUMRIENTS CONSU.ED* PER 1,000 POUNDS IIVE WEIGET DURING 1915-1916 TEST

IOT I

NAIE

Jane
Warthe 2nd
Satelite
Cap

Felibreo

Filiy Foall

Joscelin

stud Foalz

sport

Mena

$\begin{array}{rr}\text { NO. } & \text { DRY HATTER } \\ 11 & 934.205 \\ 7 & 922.023 \\ 9 & 900.969 \\ 5 & 1723.656 \\ 1 & 1457.505 \\ 17 & 1273.311 \\ 15 & 1402.813 \\ 21 & 1464.738 \\ 13 & 1551.513 \\ 3 & 1369.899\end{array}$

PROTEIN
82.906
76.514
67.860
100.658
84.147
121.789
121.926
139.231
133.544
81.476

CARBO HYDRATES

FAT

481.225

18.815

447.89319 .921

$487.307 \quad 22.569$

895.94341 .566

754.99734 .798

659.96726 .000

749.08932 .978

746.73127 .925

807.39133 .060

$709.712 \quad 32.603$
ASH

61.981

59.791

53.029

75.784

64.220

77.955

80.454

96.259

98.917

59.355

IOT II

\begin{tabular}{|c|c|c|c|c|c|c|}
\hline $\begin{array}{l}\text { J1ll } \\
\text { Ruth MoDoneld } \\
\text { Ione Rex } \\
\text { Mack } \\
\text { Forgette } \\
\text { pilig Poal } 3 \\
\text { Draft Gelding } \\
\text { Stud Foal' } \\
\text { Ruth Squirrel } \\
\text { Josephe }\end{array}$ & $\begin{array}{r}12 \\
8 \\
10 \\
6 \\
2 \\
18 \\
16 \\
22 \\
14 \\
4\end{array}$ & $\begin{array}{l}1395 \cdot 189 \\
1147.997 \\
1339.226 \\
1905.021 \\
1516.060 \\
1476.437 \\
1655.459 \\
1548.683 \\
1885.362 \\
1626.674\end{array}$ & $\begin{array}{r}153 \cdot 491 \\
126 \cdot 296 \\
147.323 \\
95 \cdot 433 \\
75 \cdot 307 \\
155 \cdot 863 \\
166.579 \\
162.153 \\
205 \cdot 824 \\
79.540\end{array}$ & $\begin{array}{l}640.260 \\
526.822 \\
614.663 \\
924.765 \\
732.218 \\
723.179 \\
766.075 \\
776.225 \\
871.926 \\
758.640\end{array}$ & $\begin{array}{l}13.801 \\
11.364 \\
13.188 \\
37.675 \\
29.778 \\
22.550 \\
23.631 \\
25.372 \\
20.075 \\
31.641\end{array}$ & $\begin{array}{r}118.270 \\
97.316 \\
113.492 \\
83.652 \\
66.381 \\
105.675 \\
114.204 \\
106.707 \\
155.955 \\
66.293\end{array}$ \\
\hline $\begin{array}{l}\text { Jo sephine } \\
\text { J1m } \\
\text { Jes8 } \\
\text { Magna } \\
\text { Lady Advanoe }\end{array}$ & $\begin{array}{l}23 \\
25 \\
27 \\
29\end{array}$ & $\begin{array}{r}1873.025 \\
1323.746 \\
1508.200 \\
891.000 \\
1263.652\end{array}$ & $\begin{array}{r}73.097 \\
79.551 \\
87.634 \\
79.714 \\
111.415\end{array}$ & $\begin{array}{l}702.030 \\
701.395 \\
768.793 \\
448.130 \\
669.629\end{array}$ & $\begin{array}{l}31.916 \\
33.734 \\
34.476 \\
17.105 \\
28.558\end{array}$ & $\begin{array}{l}9 \\
2 \\
5 \\
0 \\
9\end{array}$ \\
\hline
\end{tabular}

\footnotetext{
${ }^{1}$ Dam, Josephine, Sire, Honorable

${ }^{2}$ Dam, Ruth MoDonald, Sire Astral King

'Dam, Forgette, Sire, Honorable

4 Dam, Finette, Sire, Gardiner

5Dam, Ione Rex, Sire, Kentucky's Best

*Pounds
} 


\section{TABIE XXXXI}

SINACARY OF AVERAGE DAILY DIGESTIBLE IVURIENTS CONSULED PER 1,000 POUNDS IIVE WEIGH DURING 1915-1916 TEST

$$
\text { IOI I }
$$

MALE NO. DRY MATTER PROTEIN CARBO FAT ASH

$\begin{array}{lrrrrrr}\text { June } & 11 & 11.122 & .987 & 5.729 & .224 & .738 \\ \text { Iarthe 2nd } & 7 & 10.976 & .911 & 5.332 & .237 & .712 \\ \text { Satelite } & 9 & 10.726 & .808 & 5.801 & .269 & .631 \\ \text { Cap } & 5 & 20.519 & 1.198 & 10.666 & .495 & .902 \\ \text { Felibree } & 1 & 17.351 & 1.002 & 8.988 & .414 & .765 \\ \text { F1liy Foal } & 17 & 15.158 & 1.450 & 7.857 & .310 & .928 \\ \text { Joscelin } & 15 & 16.700 & 1.452 & 8.918 & .393 & .958 \\ \text { Stud Foal2 } & 21 & 17.437 & 1.658 & 8.888 & .332 & 1.146 \\ \text { Sport } & 13 & 18.470 & 1.590 & 9.612 & .394 & 1.178 \\ \text { Mena } & 3 & 16.308 & .970 & 8.449 & .388 & .707\end{array}$

LOT II

\begin{tabular}{|c|c|c|c|c|c|c|}
\hline 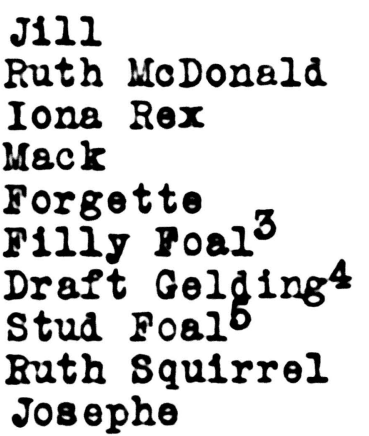 & $\begin{array}{r}12 \\
8 \\
10 \\
6 \\
2 \\
18 \\
16 \\
22 \\
14 \\
4\end{array}$ & $\begin{array}{l}16.609 \\
13.667 \\
15.943 \\
22.679 \\
18.048 \\
17.577 \\
19.708 \\
18.437 \\
22.445 \\
19.365\end{array}$ & $\begin{array}{l}1.826 \\
1.504 \\
1.754 \\
1.136 \\
.897 \\
1.856 \\
1.983 \\
1.930 \\
2.450 \\
.947\end{array}$ & $\begin{array}{r}7.622 \\
6.296 \\
7.306 \\
11.009 \\
8.717 \\
8.609 \\
9.120 \\
9.241 \\
10.380 \\
9.031\end{array}$ & $\begin{array}{l}.164 \\
.135 \\
.157 \\
.449 \\
.355 \\
.268 \\
.281 \\
.302 \\
.239 \\
.377\end{array}$ & $\begin{array}{r}1.408 \\
1.159 \\
1.351 \\
.996 \\
.790 \\
1.258 \\
1.360 \\
1.271 \\
1.857 \\
.789\end{array}$ \\
\hline $\begin{array}{l}\text { Josephine } \\
\text { Jim } \\
\text { Jes8 } \\
\text { Magna } \\
\text { Lady Advanco }\end{array}$ & $\begin{array}{l}23 \\
25 \\
27 \\
29 \\
31\end{array}$ & $\begin{array}{l}16.346 \\
15.759 \\
17.955 \\
10.607 \\
15.044\end{array}$ & $\begin{array}{r}.870 \\
.947 \\
1.043 \\
.949 \\
1.326\end{array}$ & $\begin{array}{l}8.258 \\
8.350 \\
9.152 \\
5.335 \\
7.972\end{array}$ & $\begin{array}{l}.380 \\
.402 \\
.410 \\
.204 \\
.340\end{array}$ & $\begin{array}{l}.731 \\
.700 \\
.772 \\
.697 \\
.895\end{array}$ \\
\hline
\end{tabular}

${ }^{1}$ Dam, Josephine, Sire, Eonorable

2 Dam, Ruth MoDonald, Sire, Astral King

3 Dam, Forgette, Sire, Honorable

4Dam, Finette, Sire, Gardiner

5am, Iona Rex, Sire, Zentucky's Best

*Pounds 
Tables $X X X X$ and $X X X X I$ present a summary of the total and the average dailj digestible nutrients consumed per thousand pounds live weight by the horses in the 1915-1916 test.

Lot I received silage as a part ration. Lot II was fed no silage.

The horses in this test received daily the following nutrients per thousand pounds live weight:

Dry Matter Protein Carbohydrates Fat Ash

$\begin{array}{llllll}\text { Lot I } & 15.477 & 1.203 & 8.024 & .344 & .867 \\ \text { Lot II } & 18.448 & 1.628 & 8.733 & .273 & 1.224\end{array}$

Josephine, No. 23, J1m, No. 25, Jess, No. 27, Magna, No. 29 and Lady Adrance, No. 3I, received daily, per thousand pounds 11 ve weight an average of 15.142 pounds dry matter, .425 pounds of protein, 7.813 pounds carbohydrates, .347 pounds of fat and .759 pounds of ash.

This table shows that the horses in Iot I received a dails average per head, per thousand pounds live weight 2.971 pounds dry matter, .54 pounds of protein, .709 pounds of carbohydrates and .357 pounds of ash less than Lot II.

Lot I received .071 pounds of fat more per daj than

Lot II. 
SURAARY OF THE 1914-1915 AND THE 1915-1916 TESTS

GROUP A Mature 1dle horses and mules

b. Open mares

During the 1914-1915 test there were no mares available in this division. Two mares were available during the 1915-1916 test. For tables and discussion see pages 97-99. c. Pregnant mares

During the 1914-1915 test two mares were arailable in this dirision. Four mares were available during the 1915-1910 test. There were three mares in each lot. Lot I received silage as a part ration, Lot II recolved no silage. 
TABIS XXXXII

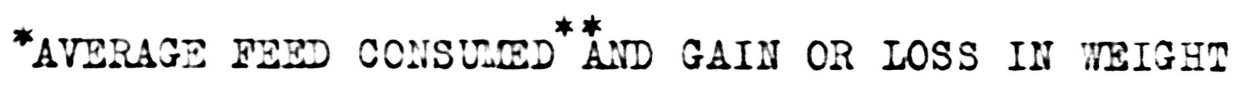

$$
I_{\text {GRAIN }} 2_{\text {HAY }} \text { SILAGE GAIN }
$$

Lot I $\quad 350.0 \quad 481.17 \quad 1131.92 \quad 97.0$

Lot II $\quad 334.671096 .25 \quad 104.0$

\begin{tabular}{|c|c|c|c|c|c|c|}
\hline & & $\operatorname{VERA}$ & DAIIY & EED CONSUEDA* & & \\
\hline & $I_{\text {GRAIN }}$ & $2_{\mathrm{H}}$ & & $A G E$ & & \\
\hline $\begin{array}{l}\text { Lot I } \\
\text { Lot II }\end{array}$ & $\begin{array}{l}4.17 \\
3.98\end{array}$ & $\begin{array}{l}6 . \\
13 .\end{array}$ & & .81 & & \\
\hline & *AVE: & $G E D$ & GESTIBIE & NUMRIENMS CONS & $\operatorname{STE} D^{* *}$ & \\
\hline & DRY MS & $\operatorname{Ter}$ & PROIEIN & CARBOHYDRATES & FAT & ASH \\
\hline $\begin{array}{l}\text { Lot I } \\
\text { Lot II }\end{array}$ & $\begin{array}{l}1203 \\
1324\end{array}$ & $\begin{array}{l}941 \\
098\end{array}$ & $\begin{array}{l}100.119 \\
145.522\end{array}$ & $\begin{array}{l}593.657 \\
609.057\end{array}$ & $\begin{array}{l}25.673 \\
13.164\end{array}$ & $\begin{array}{r}75.994 \\
109.529\end{array}$ \\
\hline
\end{tabular}

AVLRAGE DAILY DIGESTIBLE NUTRIENTS COMSUMED**

$\begin{array}{lrllll}\text { Lot I } & 14.015 & 1.192 & 7.067 & .294 & .904 \\ \text { Lot II } & 15.763 & 1.733 & 7.250 & .157 & 1.304\end{array}$

*aVarage digestible nutrieims consuided** PER 1,000 POUNDS LI VE WEIGHT

$\begin{array}{lrrrrr}\text { Lot I } & 986.029 & 81.998 & 496.206 & 21.026 & 62.240\end{array}$

Lot II $\quad \begin{array}{lllll}138.519 & 125.127 & 523.695 & 11.319 & 94.178\end{array}$

AVERAGE DAIIY DIGESTIBIE NUTRIEITS**
CONSUWED PER 1,000 POUND IIVE WEIGHM

$\begin{array}{llllll}\text { Lot I } & 11.739 & .976 & 5.907 & .250 & .741 \\ \text { Lot II } & 13.554 & 1.489 & 0.234 & .135 & 1.121\end{array}$

* For a 12 week leeding period

lCorn 2 parts, 0ats 2 parts, Bran 1 part, by weight ¿alfalfa hay

**Pounds 
Table XXXXII presents a summary of the data for Group A - c, pregnant idle mares that rere fed in both tests. There were three horses in each lot. Iot I was fed silage as a part ration. Lot II received no silage.

The table shows that Lot I received an average of .19 pounds of grain more per day than Iot II. An average of 6.69 pounds of hay was consumed by Lot I less than that consumed by Lot II. Lot I consumed per day an average of 13.81 pounds of silage. Lot II received daily 1.748 pounds of dry matter, .541 pounds of protein, .183 pounds of carbohydrates, and .40 pounds of ash per dag more than Lot $I$. Lot I was fed an average of .137 pounds of fat per day more than Lot II. When reduced to a thousand pound basis, the table shows that Lot II consumed daily 1.815 pounds of ary matter, .513 pounds of protein, .327 pounds of carbohydrates and .380 pounds of ash more than Iot I. Lot I received .115 pounds of fat per day more then Lot II.

Lot I gaired an average of 97 pounds and Lot II gained an average of 104 pounds.

GROUP B Mature horses and mules dolng average winter farm work.

\section{a. Mules}

During the 1914-1915 test two mules were available in this division. There were no mules available durirg the 1915-1916 
test.

For tables and discussion see pages 47-50

b. Geläings

Two geldirgs were aveilable during each test.

Lot I recoived silage as a part ration.

Lot II received no silage. 
TABIE XXXXIII

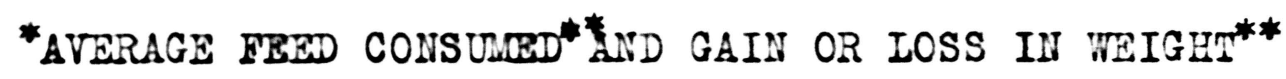
$I_{\text {GRAIN }} 2_{\text {HAY }}$ SILAGE LOSS

$\begin{array}{lrrrr}\text { Lot I } & 1151.25 & 807.75 & 1034 & 34 \\ \text { Lot II } & 1235.50 & 1374.75 & & 41\end{array}$

AVERAGE DAILY FETD CONSUNED**

$\begin{array}{lrrr}\text { Lot I } & 13.726 & 9.612 & 12.308 \\ \text { Lot II } & 14.706 & 16.353 & \end{array}$

*average digesmible nutrients consunad**

DRY MATTER PROTEIN CARBOHYDRATES FAT ASH

$\begin{array}{lllllr}\text { Lot I } & 2175.059 & 128.583 & 1108.330 & 52.403 & 96.645 \\ \text { Lot II } & 2397.164 & 186.476 & 1144.007 & 46.325 & 107.199\end{array}$

AVERAGE DAILY DIGESTIBIE NUTRIENTS CONSUARD**

$\begin{array}{llllll}\text { Lot I } & 25.894 & 1.531 & 13.195 & .624 & 1.151 \\ \text { Lot II } & 28.538 & 2.214 & 13.620 & .552 & 1.277\end{array}$

*average digesmible nUTRIEnTs consunte **

PRR 1,000 POULIDS IIVE WEIGHT

$\begin{array}{llrrrr}\text { Lot I } & 1660.350 & 98.155 & 846.054 & 40.002 & 73.775 \\ \text { Lot II } & 1843.972 & 143.212 & 880.005 & 35.635 & 82.461\end{array}$

AVERAGE DAILY DIGESTIBIE IUTRIENTS

* *ONSURED PFR 1,000 POUNDS IIVS WEIGHT

$\begin{array}{llllll}\text { Lot I } & 19.766 & 1.169 & 10.072 & .476 & .878 \\ \text { Lot II } & 21.952 & 1.705 & 10.475 & .424 & .982\end{array}$

* For a 12 week feeding period

${ }^{I}$ Corn 2 parts, oats 2 parts, bran 1 part, by weight

2 Timothy hay

**Pound 8 
Table XXXXIII presents a summery of the data for Group B - b, mature draft geldings dolng everage winter farm work, that were fed in both tests.

There were two horses in eacih lot. Iot I was fed sligge as a part ration. Lot II received no silage.

Lot I consumed daily an average of .98 pounds of grain, 6.74I pounds of hay less than Lot II. Lot I was fed an average of 12.308 pounds of silage per day.

The table of digestible nutrients consumed shows that Iot II consumed daily 2.644 pounds of dry matter, .683 pounds of protein, .425 pounds of carbohydrates and .126 pounds of ash more than Lot I. Lot I received daily .072 pounds of fat more than Lot II.

Per thousand pounds live weight, Lot I consumed 2.186 pounds of dry matter, .536 pounds of protein, .403 pounds of oarbohydrates and .104 pounds of ash per day less than Iot II. Lot I reoeived .052 pounds of fat daily more then Lot II. Lot I lost an averase of 34 pounds and Lot II lost 41 pounds.

\section{o. Pregnant mares}

Two pregnant mares were available in each test in this division.

Lot I recoived silage as a part ration. Lot II recoived no silage. 


\section{TABIE XXXXIV}

*aterage feed consunged antd gain or loss in WEIGHT ** $I_{\text {GRAIN }} 2_{\text {HAY }}$ SIIAGE GAIN

$\begin{array}{llccc}\text { Lot I } & 1202.5 & 869.75 & 912.75 & 19 \\ \text { Lot II } & 1252.5 & 1387.5 & & 42\end{array}$

AVERAGE DAILY FEED CONSUIED**

Lot I $14.313 \quad 10.353 \quad 10.861$

Lot II $14.910 \quad 16.513$

*aterage digestible nUTRIEnTs consuned**

DRY MATTER PROTEIN CARBOHYDRATES FAT ASH

$\begin{array}{lrrrrrr}\text { Lot I } & 2231.633 & 131.877 & 1130.485 & 52.307 & 99.076 \\ \text { Lot II } & 2425.099 & 126.619 & 1157.626 & 46.884 & 108.412\end{array}$

ATERAGE DAIIY DIGESTIBLE NUTRIENTS CONSUMED**

$\begin{array}{llllll}\text { Lot I } & 26.567 & 1.570 & 13.458 & .623 & 1.180 \\ \text { Lot II } & 28.829 & 1.505 & 13.781 & .558 & 1.291\end{array}$

*aterage digesmibie numrientss consuned**

PRR 1,000 POUNDS LIVE WEIGHT

$\begin{array}{llllll}\text { Lot I } & 1430.534 & 84.537 & 724.670 & 33.526 & 63.703 \\ \text { Lot II } & 1469.675 & 81.515 & 701.592 & 28.415 & 65.704\end{array}$

AVERAGE DAILY DIGESTIBIE NUTRIENIS

** CONSUMED PER 1,000 POUNDS IIVE WEIGHT

Lot I
Lot II

$\begin{array}{rr}17.030 & 1.006 \\ 17.496 & .970\end{array}$

8.627

8.352

.399

.338

.758

$17.496 \quad .970$

* For 12 week feeding period

lcorn 2 parts, oats 2 parts, bran 1 part, by weight

2 mimothy hay

**Pounds 
Table XXXXIV presents a summary of the data for Group B-O, pregnant mares performing average winter farm work, that were fed in both tests.

There were two horses in each lot. Lot I received silage as a part ration. Lot II received no silage.

Lot I consumed daily an average of .597 pounds of grain and 6.16 pounds of hay less than Lot II. Lot I was fed an arerage of 10.861 pounds of silage daily.

In amount of digestible nutrients received, Lot I was fed dally .065 pounds of protein and .065 pounds of fat more than Lot II. Lot II consumed 2.262 pounds of dry matter, .323 pounds of carbohydrates and .11l pounds of ash daily more than Lot I.

When reduced to a thousand pound basis, Lot I received an average per day of .036 pounds of protein, .275 pounds of carbohydrates and .061 pounds of fat more than Lot II. Lot II consumed daily .466 pounds of dry matter and .024 pounds of ash more than Lot $I$.

Lot I made an average gain of 19 pounds. Lot II made an average gain of 42 pounds.

GROUP C Growing horses and mules

b - I Draft forls

Two draft foals were available in each test in this division.

Lot I received silage as a part ration.

Lot II received no silage. 


\section{TABIE XXXXY}

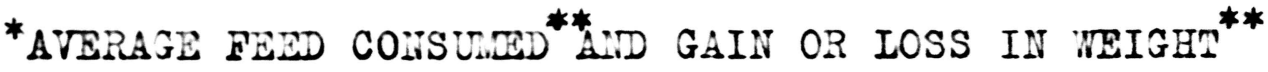
$I_{\text {GRAIN }}$ $2_{\text {HAY }}$

SIIAGE

GAIN

Lot I 425

Lot II 46 I

391.5

830.63

377.65

79

143

\section{AVERAGE DAILY FEAD COISUIED**}

Lot I

5.06

Iot II

5.49

4.66

9.884

4.499

*average digrstibie nutrienms consuned**

DRY WATTER PROTEIN CARBOHYDRATES FAT ASH

$\begin{array}{lrrrrr}\text { Lot I } & 891.875 & 80.103 & 456.152 & 18.137 & 55.757 \\ \text { Lot II } & 1193.296 & 128.311 & 569.170 & 15.281 & 88.584\end{array}$

AVERAGE DAILY DIGESTIBLE NUTRIEUTS CONSUIES H* $^{*}$

$\begin{array}{llllll}\text { Lot I } & 10.617 & 1.013 & 5.430 & .216 & .664 \\ \text { Iot II } & 14.206 & 1.528 & 6.776 & .197 & 1.055\end{array}$

*average digesmible numrienms consungd*

PER 1,000 POUNDS IIVE WEIGHT

$\begin{array}{lrrrrr}\text { Lot I } & 1087.652 & 97.686 & 556.283 & 22.118 & 67.996 \\ \text { Lot II } & 1449.934 & 115.906 & 691.579 & 18.567 & 107.635\end{array}$

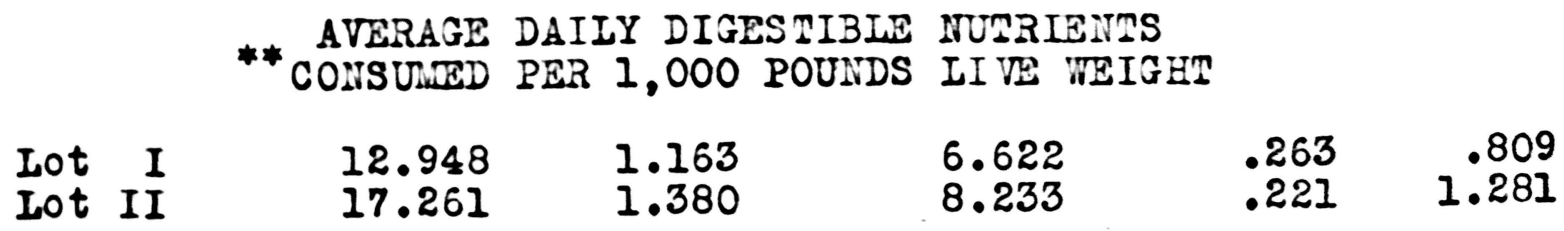

* For 12 week feoding period

$1_{\text {Corn }} 2$ parts, oats 2 parts, bran 1 part, by weight

2alfalfa hay

** Pounds 
Table XXXXV presents a summary of the data for Group c-b-l, growing draft foals that were fed in both tests. There were two foals in each lot. Lot I was fed silage as a part ration. Lot II receivea no silage. The table shows that Lot I was fed a daily average of .43 pounds of grain and 5.224 pounds of hag less than Lot II. Lot I received per day an average of 4.499 pounds of silage.

Lot II consumed daily an average of 3.589 pounds of dry matter, .515 pounds of protein, 1.346 pounds of cerbohydrates and .391 pounds of ash more than Iot I. Iot I receired a daily average of .019 pounds of fat more than Lot II. The table of digestible nutrients per 1,000 pounds live weight shows that Lot II received an average of 4.313 pounds of dry matter, .217 pounds of protein, 1.611 pounds of carbohydrates and .472 pounds of ash per day more than Lot I. Lot I was fed a daily average of .042 pounds of fat per day more than Lot II.

Lot I gained an average of 79 pounds. Lot II gained an average of 143 pounds.

\section{b - 2 Draft jearlings}

During the 1914-1915 test there were no draft yearlings arailable in this division. Two draft yearlings were available during the 1915-1916 test. For the tables and discussion see pages 121- 
c - I Fools of light breeas

There were two light foals available in each test in this division.

Lot I received silage as a part ration. Lot II received no silage. 


\section{TABIE XXXXVI}

*average med consuled*and gain or loss in weiger** $I_{\text {GRAIII }} 2_{\text {HAY }}$ SILAGE GAIN

Lot I $\quad 371.88 \quad 404.75 \quad 424.88 \quad 54$

Lot II $375.5 \quad 505.13 \quad 72$

AVERAGE DAIIY FEED CONSUIED**

$\begin{array}{llll}\text { Lot I } & 4.42 & 4.818 & 5.506 \\ \text { Lot II } & 4.421 & 6.009 & \end{array}$

*aterage digesmibie nUTRIENTS CONSUned**

DRY WATMER PROTEIN CARBOHYDRATES FAT ASH

$\begin{array}{llllll}\text { Lot I } & 872.056 & 82.969 & 442.476 & 17.230 & 56.094 \\ \text { Lot II } & 807.242 & 86.310 & 394.547 & 11.606 & 56.702\end{array}$

AVERAGB DAILY DIGESTIBLE WUTRIENTS CONSURED**

Lot I

10.382

.989

5.268

.205

.668

Iot II

9.611

1.028

4.697

.136

.675

*average digestibie humrients consunard** PER 1,000 POUNDS IIVE WEIGHT

$\begin{array}{lllllr}\text { Lot I } & 1495.808 & 142.314 & 758.964 & 29.554 & 96.216 \\ \text { Lot II } & 1543.484 & 165.029 & 754.201 & 22.191 & 108.417\end{array}$

AVERAGE DAILY DIGESTIBIE NUTRIENTS

** CONSUMED PER 1,000 POUNTS IIVE WEIGHT

$\begin{array}{llllll}\text { Iot I } & 17.807 & 1.694 & 9.035 & .352 & 1.145 \\ \text { Lot II } & 18.375 & 1.965 & 8.979 & .264 & 1.291\end{array}$

* For 12 week leoding period

${ }^{1}$ Corn 2 parts, oats 2 parts, bran 1 part, by weight

2 Alfalfa

**Pound 8 
Table XXXXVI presents a summary of the data for Group C-1, growing light foals that were fed in the test. There were two horses in each lot. Iot I received sllage as a part ration, lot II received no silage. These lots were fed practically the same amounts of grain per day. Lot I was fed an average of .19I pounds of hay less per day than Lot II. Iot I received an average of 5.506 pound of silage.

The table of digestible nutrients shows that lot II received an average of .039 pounds of protein and .007 pounds of ash daily more than Lot I. Lot I consumed per day an average of .77I pounds of dry matter, .571 pounds of carbohydrates and .069 pounds of fat more than Lot II.

Then reduced to a thousand pound basis, Lot I received a daily average of .568 pounds of dry matter, .27I pounds of protein and .146 pounds of ash per day less than Lot II. Lot I consumed an average per day of .056 pounds of carbohydrates and .088 pounds of fat more than Lot II.

Lot I gaired an average of 54 pounds. Iot II gained an average of 72 pounds.

c - 2 Yearlings of light breeds During the 1914-1915 test there were no yoarlings available in this division. Fror the tables and discussion of Pepper, No. 15 a two year old and Lady Advance, No. 16 , 
a yearling, see pages $71-74$.

Two light yearlings vere available durirg the 1915-1916 test.

for tre tables and discussion see pages 127-129.

GROUP D Immature horses and mules performing average

wirter farm work.

b. Open mares

Durirg the 1914-1915 test there were two

mares available in this division. There

were no mares available during the 1915-1916

test. For the discussion see page 74 .

c. Pregnant mares

During the 1914-1915 test there were no mares available in this division. Two mares were available during the 1915-1916 test. fror the tables and discussion see pages 130-133.

GROUP $\mathrm{B}$ Immature idie horses and mules

c. Pregnant mares

During the 1914-1915 test there were two mares available in this division. There were no mares available during the 1915-1916 test.

For the tables and discussi on see pages 75-78. 
SUSCLARY

Corn s1lage of good quality was fed to horses of various ages under varying conditions during the two tests.

One mature mule performing average winter farm work was fed silage during the first test.

Large quantities of silage were not eaten at first, but when the horses became accustomed to it larger amounts were consumed.

Sllage was consumed in amounts of from an average of 3.997 pounds dailg in case of Filly Foal, No. 17 during 1915-1916 to an average of 15.908 pounds in case of ide pregrant mare, Satelite, No. 9 during the same test.

It should not be concluded that this is the maximum amount of silage that oan be fed.

At the close of the tests twelve "silage fed horses" were in better thrift than those fed no silage. In three cases there was no noticeable difference. In four cases the "silege fed horses" Indicated slightlg less thrift than those recelving only grain and hay. In the latter oase two of the horses fod sllage lacked thrift at the beginning of the test. An average of the digestible nutrients consumed daily per thousand pounds live weight for both tests show that the "Bllage fed herses" received daily less ary matter, protein, carbohydrates and ash than the horses fed no silage and that they received slightly more fat. 
One open idle mare was fed an average daily ration of 4 pounds grain, 5 pounds of alfalfa hay and 8.833 pounds of silage during the 1915-1916 test. A check lot was fed daily 4 pounds grain and 14.5 pounds of alfalfa hay. The silage fed mare lost 18 pounds and the check lot gained 18 pound during the test. There was no noticeable difference in the condition or thrift of these mares at the close of the test.

Three idle pregnant mares were fed an average daily ration of 4.17 pounds grain, 6.42 pounds alfalfa hay and 13.81 pounds of 81 lage during the feeding period. Three mares, fed as a cheok lot, consumed daily 3.98 pounds grain and 13.11 pound of alfalfa hay. The silage fed mares gained an average of 97 pounds. The mares in the check lot made an average gain of 104 pounds. At the close of the test two of the "81lage fed mares" were in better thrift than those not fed 81lage. The thrift of one of the silage fed mares was slightly inferior to the thrift of the check lot.

The pregnant mares fed silage produced foals that were as large, straight and active as the pregnant mares fed only grain and alfalfa haj.

One mule performing aversge winter farm work was fod an average dally ration of 6.22 pounds grain, 8.12 pounds of timothy hay and 8.05 pounds silage during the 1914-1915 test. The mule fed as a check consumed daily 7.76 pounds grain 
and 12.31 pounds of timothy hay. The silage fed mule lost 45 pounds during the test. The mule fed no silege lost 5 pounds. At the close of the test the silage fed mule was in much better thrift than the other mule as was indicated by the condition of the hair and skin.

Two geldings performing average wirter farm work were fed an average dailg ration of 13.726 pourds grain, 9.17 pounds timothy hay and 12.308 pounds of silage. Two geldings fed as a check consumed a daily arerage of 14.706 pounds grain and 16.350 pounds of timothy hay. The silage fed geldings lost an everage of 34 pound in weight. The geldings fed as a check lot lost 41 pounds in weight. Both silage fed geldings were in better thrift at the close of the test than the geldings fed only grain and timothy hay.

Two mature pregnant mares doing average wirter farm work were fed an average daily ration of 14.313 pounds grain, 10.353 pounds of timothy has and 10.861 pounds of silage. Two mature pregnant mares fed as a chock consumed a daily averege of 14.91 pounds of grain and 16.513 pounds of timothy haj. The mares fod silage made an average gain of 19 pounds and the mares fed no silage gained an average of 42 pounds during the feeding perlod. The silage fed rares were in better thrift at the close of the test than the mares fed no 81lage. The produced foals that were as large, strong, otraight and active as were produced by the mares led only grain and timothy hay. 
Two draft foals were fod an average daily ration of 5.06 pounds grain, 4.66 pounds alfalfa hag and 4.499 pounds of silage. Two foals fed as a check lot consumed a daily average of 5.49 pounds grain and 9.884 pounds alfalfa hay. The foals fed silage made an averoge gain of 79 pounds and the foals fed only grain and hay gained an average of 143 pounds during the test. The silage fed fols were in slightlg better thrift at the close of the test.

One jearling draft filly was fed a daily ration of 10 pounds grain, 5.667 pounds of alfalfa hay and 13.604 pounds of silage. One jearling gelding fed as a check received daily an average of 10 pounds grain and 14.5 pounds alfalfa hay. The "sllage fed yearling" gained 50 pounds and the gelding fed only grain and alfalfa gained 92 pounds during the test. At the olose of the test the yearling fed silege was in better thrift then the gelaing fed no silage.

Two light foals were fed an average daily ration of 4.42 pounds grain, 4.818 pounds of alfalfa hay and 5.506 pounds of silage. The check lot were fed daily 4.421 pound grain and 6.009 pounds of alfalfa hay. The foals receiving silage made an average gain of 54 pounds and the two foals fed only grain and alfalfa made an average gain of 72 pounds during the test. In one case the silage fed foal indicated better thrift and in the other oase the foal fod no silage was in better thrift at the close of the test. 
One saddle yearling was fed an average dally ration of 4.667 pounds grain, 4.9 pourds alfalfa hay and 10.795 pounds of silage. One jearling fed as a check lot consumed daily 4.667 pounds of grain and 14.315 pounds of alfalfa hay during the second trial. The yearling fed only grain and alfalfa hay was in slightly better thrift at the close of the test. One light two year old was fed an average daily ration of 2.07 pounds grain, 5.88 pounds alfalfa hay and 10.27 pounds silage. The check lot, a jearling, was fed a daily ration of 4 pounds grain and 8.86 pourds of alfalfa haj. The two year oid fed silage gaired 39 pounds during the test and the yearling fed as a check gained 35 pounds. The silage fed two year old was in better thrift at the close of the test than the yearling fed only grain and alfalfa hay.

One immature idle pregnant mare was fed an average dally ration of 3.93 pounds grain, 5.18 pounds alfalfa hay, and 9.28 pounds of 811 ge. One immature idle pregnant mare fed as a check received daily 4 pounds grain and 14.42 pounds alfalfa hay. The mare fed silage gained 73 pounds during the test and the check fed no silage gaired 96 pounds. The silage fed mare was in much better thrift at the close of the test. One immature open mare performing average winter farm work refused the sllage at first. After the quantity of grain and hay led was reduced she ate the silage. She was consuming about 8 pounds of silage per dey when she developed a 
case of destemper and indigestion and had to be taken off the experiment.

One immature pregnant mare performing average winter farm work was fed an average ration of 14.286 pounds grain, 8.637 pounds of timothy hay and 9.896 pounds of silage. One immature pregnant mare performing average winter farm work fod as a check lot recoived daily 15.131 pounds of grain, and 17.321 pounds of alfalfa hay. The silgge fed mare gained 40 pounds and the check fed only grain and hay gained 3 pounds during the feeding period. The hair and skin of the silage fod mare indicated that she was in better thrift then the mare fed no 81lage. The mare fed sllage dropped a large, active foal soon after the test closed.

The following are the rations fed and the gain or $108 \mathrm{~s}$ in weight of the horses fed with no check lot during the 1915-1916 test:

The mature idle open mare Josephine, No. 23 was fed an average daily ration of 11.427 pounds of grain, 9.238 pounds of timothy hay and 13.402 pounds of silage. This mare lost 5 pounds during the test.

The mature idle gelding Jim, No. 25 was fed on average daily ration of 11.489 pounds grain, 10 pounds of timothy hay and 15.136 pounds of silage. This gelding did not gain or lose in weight during the test.

The open mare Jess, No. 27, performing delivery work, wes fed an arerage daily ration of 14.405 pounds grain, 9.900 
pounds of timothy hay and 6.098 pounds of silge. This mare lost 50 pounds in weight during the test. The mature idle open mare liagna, 10. 29 was fed an averge daily ration of 4 pounds of grain, 5 pounds of alfalfa hay and 7.205 pounds of silage. This mare lost 16 pounds during the test.

The immature two year old filly Lady Advance, No. 31 was fed an average daily ration of 7.333 pounds grain, 5 pounds of alfalfa hay and 10.262 pounds of silage. This mare lost 12 pounds during the test. She was driven some. These horses were ell in good thrifty condition at the close of the feoding period. The mature idle pregnant mare Satelite, No. 9 and the mature idle gelding Jim, No. 25 were led only grain and silage during the last five weeks of the 1915-1916 test. 


\section{CONCLUSION}

This was an initial effort to feed silage as a part ration to horses and mules of various ages and under varying conditions at the Missouri Experiment station.

The data in the 1915-1916 tests are not sufficient to make possible conclusions for each of the groups.

Corn silage of good quality, made from comparatively mature corn, properly put into the silo, containing no moldy, frozen or rotten parts may be fed in limited quantities to horses of various ages under varying conations. 


\section{SUGGESTIONS FOR FUTURE INVESTIGATIONS}

1. Definite conclusions concerning the most satisfactory combination of corn silage and other feeds for horses and mules of various ages and under varying conditions are not possible until more data and information are available for the various groups here outlined. Further data, therefore, should be secured for each class of horses.

2. The data from the two trials presented indicate that the horses were being maintained in medium growing, working and breeding condition. No effort was made to fatten the horses. If possible, further data should be secured to determine whether relatively more corn silage may be utilized in the ration for production of fat in mature horses and mules, and to procure more rapid growth and development in growing horses and mules.

3. Wolds and bacterial growths frequently found in silage and the effect of each upon horses and mules should be studied. If it is found that any or all of these cause sickness or other undesirable results, means for counteracting these effects should be sought..

4. The problem of using silage in the ration for fattening horses and mules for market was not included in the outline. Data and information should be procured on this important phase of horse production.

5. The most noticeable result observed in the two trials 
discussed was the more healthy and thrifty condition of the hair and skin in the "silage fed horses." "Ihis succulent feed tends to keep the bowels normal, the body tissues sappy, the skin pliant and the coat 8lossy, all of which mark the animals as in condition to make the most from their feed."

6. Further efforts should be made to discover the maximum amount of silage that can be safely and economically fed to horses and mules of various ages and under varying conditions.

7. A study of the costs of standard rations containing different amounts of silage should be made.

8. Data should be secured on the possibilities of feeding silage as a sole roughage with grain to mature idle horses and any other groups of horses not requiring feeds in concentrated form.

9. A study should be made of the possibility of maintaining idle horses and mules on a ration of silage and hay. 10. A study should be made of the effect of silage as a part ration during the winter on the ability of horses to pasture the following summer.

11. Observations should be made of the excretion, appetite, opirit and ablitty to perform work of horses and mules fed

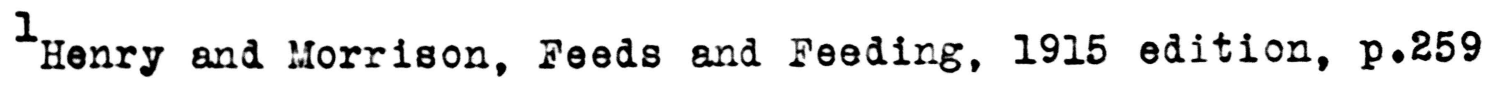


silage as a part ration. In case of brood mares a study should be made of the effect of silage in the ration on the development of the fetus and the growth of the foal when suckling its dam.

12. The coefficient of digestibility should be determined for sllage when fed to horses and any other means of judging results should be studied. 


\section{ACKNONLEDGiEvin}

The writer desires to express his indebtedness to Professor E. A. Mrombriage of the Animal Husbandry Department of the University of ifissourl for his suggestions and advice, and to many others who have given valued assistance in the preparation of this paper. 


\section{UNIVERSITY OF MISSOURI}

Columbia

Dean irater vilier.

Chal rman, Greduate Committeo.

Dear Dean Mller:

The dissertation of $\mathrm{Kr}$. Elmer Eughes on "Silage as

a Part Retion for Horses and hales of Various Ages and under Varjing Conditions" is in my opinion worthy of accoptance, in partial fulfillment of the requirements for the degree of Master of Arti.

The dissertation is based, In pert, on an incomploted teeding experiment in a rield of researon in waich but Iittle had previously been done; and in the neture of the $088 \theta$ considerable work must jet be done before the definite results sought for are obtained. But the excellent presentation of the plan and object of the research with the tabalations, 1llustrations, and analysis of the data showing present status of the research. I think quite fallg meot the requirement8. I therefore join with Prof. B. A. Trowbridge in recomending the acoeptance of the dissertation. Very truly.

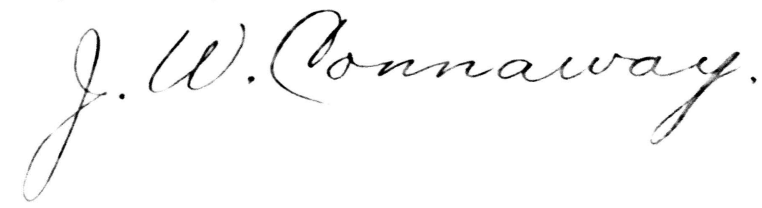


$378.7 \mathrm{M71}$
$\times 1875$

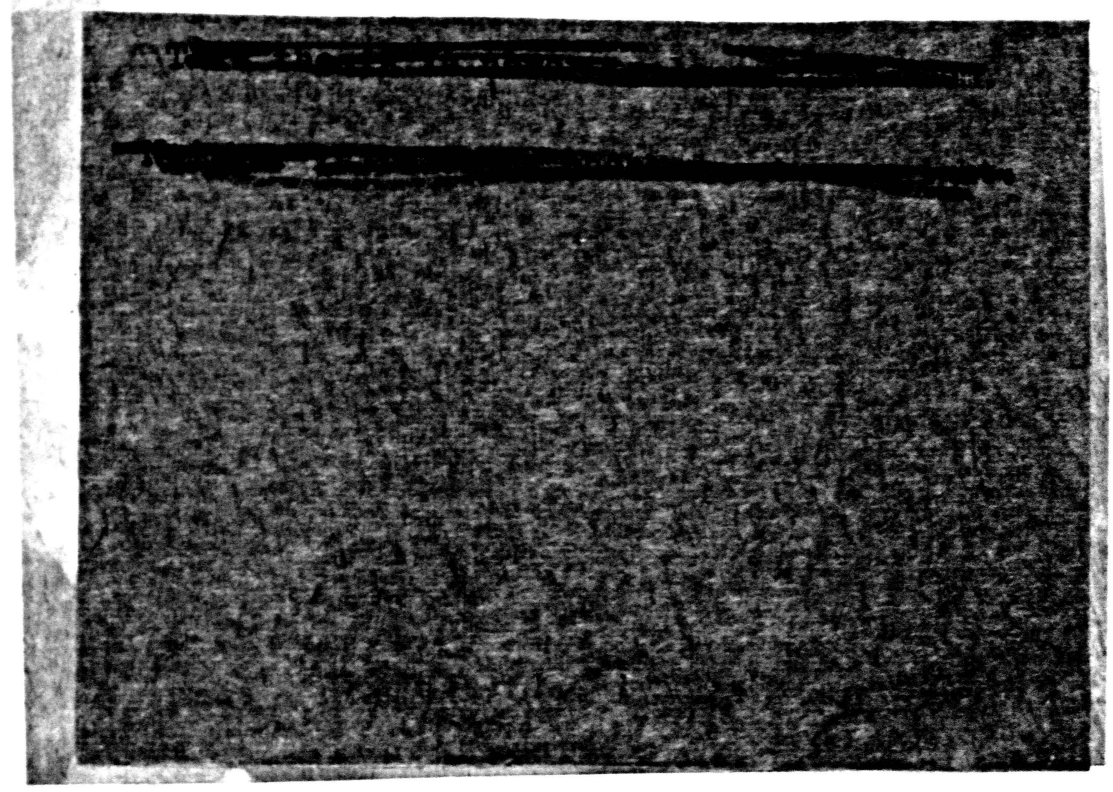


MU Libraries

HughesEI mer 1916 Specsheet, t $x t$

University of Mi ssouri-.-Col umbia

MU Theses and Dissertations (Pre-1920)

Local identifier

Capture information

Date captured

Scanner manufacturer

Scanner model

Scanning system software

Optical resolution

Color settings

File types

Source information

For mat

Content type

Source I D

Notes

Hughes EI mer 1916

20160914

Zeutschel

OS 15000

Omniscan v.12.4 SR4 (1947) 64-bit

$600 \mathrm{dpi}$

grayscale, 8 bit and color, 24 bit

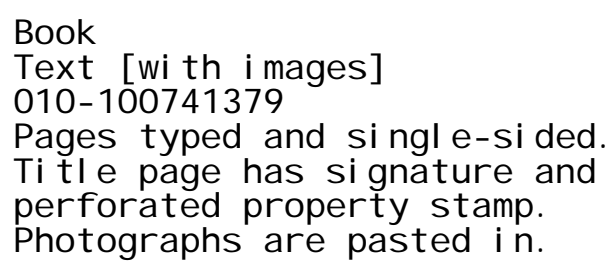

$010-100741379$

Pages typed and single-sided.

Title page has signature and

perforated property stamp.

Photographs are pasted in.

Derivatives - Access copy

Compression

Editing sof tware

Resolution

Color

File types

Notes
Tiff compressed with LZW before conversion to pdf Adobe Photoshop CS5 $600 \mathrm{dpi}$

Grayscale and color $p d f$

Grayscale pages cropped, canvassed, and i mages brightened.

Col or pages cropped.

Bl ank pages removed. 